\title{
Glättungsmechanismen beim Ionenbeschuss rauer amorpher Oberflächen
}

\author{
Dissertation \\ zur Erlangung des Doktorgrades \\ der Mathematisch-Naturwissenschaftlichen Fakultäten \\ der Georg-August-Universität zu Göttingen
}

vorgelegt von

Sebastian Vauth

aus Rahden

Göttingen 2007 
D7

Referent:

Prof. Dr. S. G. Mayr

Korreferent:

Prof. Dr. H.-U. Krebs

Tag der mündlichen Prüfung:

11.10.2007 


\section{Inhaltsverzeichnis}

1 Einleitung 1

2 Experimentelles $\quad 4$

2.1 Die Ultrahochvakuum-Anlage . . . . . . . . . . . . . . . . . . . 4

2.2 Probenherstellung und Eigenschaften . . . . . . . . . . . . . . 6

2.2.1 Materialeigenschaften . . . . . . . . . . . . . . . 6

$2.2 .2 \quad$ Schichtherstellung . . . . . . . . . . . . . . . . . . . . . . . 7

2.2 .3 Ionenbestrahlung . . . . . . . . . . . . . . . . . . . . 8

2.3 Analysemethoden . . . . . . . . . . . . . . . . . . . . 10

2.3.1 Rastertunnelmikroskopie . . . . . . . . . . . . . . . . . . 10

2.3.2 Röntgendiffraktometrie und -reflektometrie . . . . . . . . . . . . . 11

2.3.2.1 Kleinwinkelröntgenreflektometrie . . . . . . . . . . . 11

2.3.2.2 Weitwinkelröntgendiffraktometrie . . . . . . . . . . . 12

2.3.3 Rutherford-Rückstreuung . . . . . . . . . . . . . . . . 13

2.3.4 Auswertung experimenteller Daten . . . . . . . . . . . . . 13

3 Molekulardynamik-Simulationen $\quad \mathbf{1 6}$

3.1 Ziel der Molekulardynamik-Simulationen . . . . . . . . . . . . . . . . 16

3.2 Grundlagen . . . . . . . . . . . . . . . . . . . . . . 16

3.3 Eigenschaften des verwendeten Simulationsprogramms . . . . . . . . . . 18

3.3.1 Druck- und Temperaturkontrolle . . . . . . . . . . . . . . . 18

3.3.2 Wechselwirkungspotentiale . . . . . . . . . . . . . . . . 20

3.3.2.1 Das CuTi Potential . . . . . . . . . . . . . . . . 20

3.3.2.2 Das Si Potential . . . . . . . . . . . . . . 21

3.3.2.3 Zusätzliche Wechselwirkungen beim Einschuss von Atomen 23

3.4 Probenherstellung . . . . . . . . . . . . . . . . 23

4 Kontinuumsmodellierung 26

4.1 Grundlagen . . . . . . . . . . . . . . . . . . . . . . 26

4.2 Kontinuumsmodellierung und Skalenkonzepte . . . . . . . . . . . . . . . 27

4.3 Kontinuumsmodell im Fourierraum . . . . . . . . . . . . . . . . . . . . . 28

4.4 Schichtwachstum $\ldots \ldots \ldots \ldots$. . . . . . . . . . . . . . . . . . . . 29

4.5 Modifikation der Oberflächenstruktur durch Ionenbestrahlung . . . . . . 31

4.5.1 Sputtererosion . . . . . . . . . . . . . . . . . . . 31

4.5.2 Oberflächendiffusion . . . . . . . . . . . . . . . . . . . 32

4.5.3 Ballistischer Transport . . . . . . . . . . . . . . 33 
4.5 .4 Viskoses Fließen . . . . . . . . . . . . . . . . . . . . . 34

4.5.4.1 Viskoses Oberflächenfließen . . . . . . . . . . . 35

4.5.4.2 Viskoses Volumenfließen . . . . . . . . . . . . . . 36

4.5 .5 Nichtlokale Prozesse . . . . . . . . . . . . . . . . . . . . 37

4.5.6 Stochastisches Rauschen . . . . . . . . . . . . . . . . . 37

4.6 Relatives Gewicht der Prozesse . . . . . . . . . . . . . . 38

4.7 Einfluss der Glättungsprozesse auf die Entwicklung der Rauigkeit . . . . 38

4.8 Numerische Lösung der Kontinuumsmodelle . . . . . . . . . . . . . 40

5 Experimentelle Ergebnisse $\quad 41$

5.1 Oberflächentopographien unter dem Einfluss von Ionenbestrahlung . . . . 41

5.2 Quantitative Auswertung der Oberflächentopographien . . . . . . . . . 49

5.3 Analyse der Schichtdicke und Zusammensetzung . . . . . . . . . . . . 53

5.4 Struktur der bestrahlten Probe $\ldots \ldots \ldots \ldots \ldots \ldots$

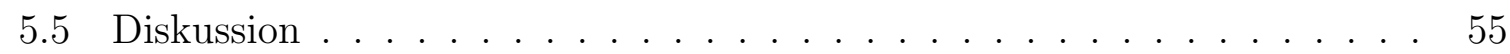

5.5 .1 Entwicklung der Oberflächenstruktur . . . . . . . . . . 55

5.5.2 Schichtdicke, Zusammensetzung und Struktur der Probe . . . . 59

6 Thermisch aktivierte Prozesse in der MD-Simulation $\quad 61$

6.1 CuTi Legierungen . . . . . . . . . . . . . . . . . . . . 61

6.1.1 Herstellung, Struktur und Glasübergang . . . . . . . . . . . 62

6.1.1.1 Thermodynamische Größen und Glasübergang . . . . . . 62

6.1.1.2 Atomare Struktur und Ordnung . . . . . . . . . . . . . 64

6.1 .2 Diffusionsprofile der CuTi Schichten . . . . . . . . . . . . . 67

6.1.3 Verteilungsfunktion atomarer Verschiebungen . . . . . . . . 71

6.1 .4 Energieverteilung . . . . . . . . . . . . . . . . . 73

6.1.5 Atomare Dynamik bei unterschiedlichen Verschiebungsweiten . . . 75

6.2 Diskussion der Ergebnisse zum thermisch aktivierten Verhalten von CuTi

Schichten . . . . . . . . . . . . . . . . 77

6.2.1 Probenherstellung und Charakterisierung . . . . . . . . . . . 77

6.2 .2 Untersuchung der atomaren Dynamik . . . . . . . . . . . . . . 80

6.3 Amorphes $\mathrm{Si} \ldots \ldots \ldots \ldots \ldots \ldots \ldots$. . . . . . . . . . . . . 86

6.3.1 Herstellung, Struktur und Glasübergang . . . . . . . . . . 86

6.3.1.1 Thermodynamische Größen und Glasübergang . . . . . . 86

6.3.1.2 Strukturanalyse der Si Proben . . . . . . . . . . . . . 87

6.3 .2 Dynamik der Si Schichten . . . . . . . . . . . . . . . . . . . . 89

6.3 .3 Energieverteilung in Si Schichten . . . . . . . . . . . . . 93

6.4 Diskussion des thermisch aktivierten Verhaltens von amorphem Si . . . . 94

6.4.1 Probenherstellung und Charakterisierung . . . . . . . . . . . 94

6.4.2 Dynamik dünner Si Schichten . . . . . . . . . . . . . . . . . 98

6.5 Zusammenfassende Diskussion . . . . . . . . . . . . . . . 100 
7 Bestrahlungsinduzierte Prozesse in der MD-Simulation 102

7.1 Bestrahlung von amorphen CuTi Oberflächen . . . . . . . . . . . . . 103

7.1.1 Allgemeine Phänomene . . . . . . . . . . . . . . . . . . 103

7.1.2 Analyse der atomaren Dynamik . . . . . . . . . . . . . . . . 106

7.1.3 Identifikation der Parameter aus Kontinuumsmodellen . . . . . . 108

7.1.3.1 Physikalische Einheiten bei Bestrahlungsprozessen . . . 108

7.1.3.2 Viskoses Oberflächenfließen und Oberflächendiffusion . . 110

7.1.3.3 Ergebnisse zum Vergleich von viskosem Oberflächenfließen und Oberflächendiffusion . . . . . . . . . . . 115

7.1.3.4 Viskoses Oberflächenfließen und ballistischer Transport . 119

7.1.3.5 Ergebnisse zum Vergleich von viskosem Oberflächenfließen und ballistischem Transport . . . . . . . . . . . . 122

7.1.3.6 Vergleich von glättenden und aufrauenden Mechanismen 123

7.2 Diskussion der Ergebnisse zur Bestrahlung amorpher CuTi Oberflächen . 125

7.2 .1 Allgemeine Phänomene . . . . . . . . . . . . . . . . 125

7.2.2 Analyse der atomaren Dynamik . . . . . . . . . . . . 126

7.2.3 Untersuchung der Prozesse im Rahmen von Kontinuumsmodellen 127

7.3 Bestrahlung amorpher Si Oberflächen . . . . . . . . . . . . . . . . . . . 134

7.3.1 Allgemeine Phänomene . . . . . . . . . . . . . . . . . . . 134

7.3.2 Analyse der atomaren Dynamik . . . . . . . . . . . . . . . 135

7.3.3 Identifikation der Parameter aus Kontinuumsmodellen . . . . . . 135

7.3.3.1 Viskoses Oberflächenfließen und Oberflächendiffusion . . 135

7.3.3.2 Viskoses Oberflächenfließen und ballistischer Transport . 137

7.3.3.3 Vergleich von glättenden und aufrauenden Mechanismen 139

7.4 Diskussion der Ergebnisse zur Bestrahlung amorpher Si Oberflächen . . . 139

7.4.1 Allgemeine Phänomene und atomare Dynamik . . . . . . . . . . . 139

7.4.2 Untersuchung der Prozesse im Rahmen von Kontinuumsmodellen 140

7.5 Zusammenfassende Diskussion . . . . . . . . . . . . . . . . . . . . . 144

8 Ergebnisse der Simulationen von Kontinuumsmodellen $\quad 146$

8.1 Oberflächenfließen und Sputtererosion . . . . . . . . . . . . . . . . . . . 147

8.2 Oberflächenfließen und ballistisches Glätten . . . . . . . . . . . . . . . . 149

8.3 Nichtlokale Prozesse . . . . . . . . . . . . . . . . . . . . . . 153

8.4 Modellierungsansätze zum Volumenfließen . . . . . . . . . . . . . . 155

8.5 Oberflächenfließen, ballistisches Glätten und Volumenfließen . . . . . . . 159

8.6 Diskussion . . . . . . . . . . . . . . . . . . . . . . . . 161

9 Diskussion 165

9.1 Ergebnisse der angewendeten Untersuchungsmethoden . . . . . . . . 165

9.2 Vergleich mit anderen Materialsystemen . . . . . . . . . . . . . . . . . . 170

10 Zusammenfassung und Ausblick $\quad 172$ 
11 Anhang $\quad 174$

11.1 Oberflächenfließen im Ortsraum . . . . . . . . . . . . . . . . . . . . 174

11.2 Einfluss der Glättungsprozesse auf die Entwicklung der Rauigkeit . . . . 176

11.3 Beitrag des Rauschens zur Rauigkeit . . . . . . . . . . . . . . . . . 178

11.4 Volumenfließen im Ortsraum . . . . . . . . . . . . . . . . . . . . . . . . . 179

$\begin{array}{ll}\text { Literaturverzeichnis } & 181\end{array}$

\begin{tabular}{ll}
\hline Publikationen & 193
\end{tabular}

$\begin{array}{ll}\text { Danksagung } & 194\end{array}$

\begin{tabular}{ll}
\hline Lebenslauf & 195
\end{tabular} 


\section{Abbildungsverzeichnis}

2.1 Schematische Darstellung der UHV-Anlage. . . . . . . . . . . . . . 5

2.2 Temperaturintervall $\Delta T_{x}$ der unterkühlten Schmelze im ternären Phasendiagramm von $\mathrm{ZrAlCu}$ (nach [IZM92]; die gestrichelte Linie begrenzt den Bereich der amorphen Phase). . . . . . . . . . . . . . . 7

2.3 Ionenstrahlprofil der „Ion Bombardment Gun 981-2043“ entlang der Richtungen senkrecht zur Strahlrichtung. . . . . . . . . . . . . . . .

3.1 Schematische Darstellung der Probenherstellung in der MD-Simulation im Temperatur-Zeit Diagramm. . . . . . . . . . . . . . . .

3.2 Zelle für die Simulation dünner Schichten; an weißen Fläche herrschen periodische und an orangen Flächen offene Randbedingungen. . . . . . . 25

4.1 Diffusive Bewegung von Oberflächenatomen. . . . . . . . . . . . . . . . . 32

4.2 Ballistischer Glättungsprozess. . . . . . . . . . . . . . . . . . . . . 34

4.3 Viskoses Fließen a.) in einer dünnen Oberflächenschicht, b.) in einem ausgedehnten Volumenbereich . . . . . . . . . . . . . . 35

5.1 STM Bilder und zugehörige Höhen-Höhen-Korrelationsfunktionen für verschiedene Fluenzen (Bildgröße: $L=200 \mathrm{~nm}$, Einheit der Höhenskala: nm). 43

5.2 STM Bilder und zugehörige Höhen-Höhen-Korrelationsfunktionen für verschiedene Fluenzen (Bildgröße: $L=200 \mathrm{~nm}$, Einheit der Höhenskala: nm). 44

5.3 STM Bilder und zugehörige Höhen-Höhen-Korrelationsfunktionen für verschiedene Fluenzen (Bildgröße: $L=200 \mathrm{~nm}$, Einheit der Höhenskala: nm). 45

5.4 STM Bilder und zugehörige Höhen-Höhen-Korrelationsfunktionen für verschiedene Fluenzen (Bildgröße: $L=200 \mathrm{~nm}$, Einheit der Höhenskala: nm). 46

5.5 STM Bilder und zugehörige Höhen-Höhen-Korrelationsfunktionen für verschiedene Fluenzen (Bildgröße: $L=200 \mathrm{~nm}$, Einheit der Höhenskala: nm). 47

5.6 STM Bilder und zugehörige Höhen-Höhen-Korrelationsfunktionen für verschiedene Fluenzen (Bildgröße: $L=400$ nm, Einheit der Höhenskala: nm). 48

5.7 Rauigkeit als Funktion der Fluenz für zwei verschiedene Bildgrößen a.) in linearer Auftragung und b.) in doppeltlogarithmischer Auftragung. . . . . 49

5.8 a.) Korrelationslängen $R_{e}$ und b.) Rauigkeitsexponenten $\chi$ als Ergebnis aus den STM-Daten für verschiedene Bildgrößen. . . . . . . . . . . . 50

5.9 Höhen-Differenz-Korrelationsfunktionen der STM Bilder aus Abb. 5.1-5.5. 51

5.10 Spektrale Leistungsdichten für verschiedene Fluenzen. . . . . . . . . . . . 52

5.11 Spektrale Leistungsdichten für a.) die gewachsene Schicht und b.) die glatteste Schicht. . . . . . . . . . . . . . . . . . . 53 
5.12 Exponent des Zerfalls der spektralen Leistungsdichten als Funktion der Fluenz für verschiedene Bildgrößen. . . . . . . . . . . . . . . . .

5.13 Signal der Rutherford-Rückstreuung (schwarz) und mit RUMP [Com04] simulierte Kurve (rot) für den Bereich in der Mitte der Probe.

5.14 Weitwinkel-Röntgenmessung der $\mathrm{Zr}_{65} \mathrm{Al}_{7.5} \mathrm{Cu}_{27.5}$ Schicht nach der Bestrahlung mit einer Fluenz von $\Phi=4 \cdot 10^{17}$ Ionen $/ \mathrm{cm}^{2}$. . . . . . . . . . . . . .

6.1 a.) Volumen und b.) Energie jeweils in Abhängigkeit der Temperatur für $\mathrm{Cu}_{50} \mathrm{Ti}_{50}$ im Bereichs $\mathrm{T} \leq 1000 \mathrm{~K}$ beim Abkühlen mit linearen Fits (Rate:

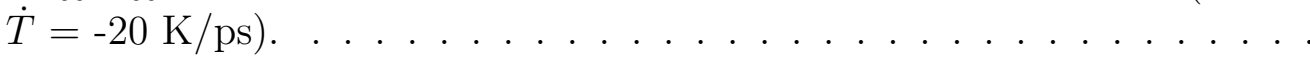

6.2 Paarkorrelationsfunktion von $\mathrm{Cu}_{50} \mathrm{Ti}_{50}$ direkt nach dem Abschreckprozess bei $T=0 \mathrm{~K}$ ohne Oberflächen (schwarze Linie) und nach Abschluss der Diffusionsuntersuchungen über 500 ps bei $T=450 \mathrm{~K}$ mit Oberflächen in einer Richtung (rote Linie). . . . . . . . . . . . . . . . . . .

6.3 Paarkorrelationsfunktion von a.) $\mathrm{Cu}_{40} \mathrm{Ti}_{60}$ und b.) $\mathrm{Cu}_{60} \mathrm{Ti}_{40}$ jeweils direkt nach dem Abschreckprozess bei $T=0 \mathrm{~K}$ ohne Oberflächen (schwarze Linie) und nach Abschluss der Diffusionsuntersuchungen über 500 ps bei $T=450 \mathrm{~K}$ mit Oberflächen in einer Richtung (rote Linie). . . . . . . . . 66

6.4 Tiefenprofile der Zusammensetzung der CuTi Schichten. . . . . . . . . . 69

6.5 Tiefenprofile der Diffusionskoeffizienten der $\mathrm{CuTi}$ Schichten: a.) $\mathrm{Cu}_{40} \mathrm{Ti}_{60}$, b.) $\mathrm{Cu}_{50} \mathrm{Ti}_{50}$ und c.) $\mathrm{Cu}_{60} \mathrm{Ti}_{40} \ldots \ldots \ldots \ldots$. . . . . . . . . .

6.6 Verteilung atomarer Verschiebungen in $\mathrm{Cu}_{50} \mathrm{Ti}_{50}$ im Probeninneren und im Oberflächenbereich für a.) $\mathrm{Cu}$ und b.) Ti. . . . . . . . . . . . . . . . .

6.7 Verteilung potentieller Energien der einzelnen Atome in der gesamten $\mathrm{Cu}_{50} \mathrm{Ti}_{50}$ Probe bei $\mathrm{T}=450 \mathrm{~K}$ für a.) $\mathrm{Cu}$ und b.) $\mathrm{Ti}$ (jeweils schwarze Linie). Die rote Linie stellt eine angepasste Gauß - Normalverteilung dar.

6.8 Positionen der Atome mit potentieller Energie $E_{p o t}>E_{M}+2 \sigma_{E}$ als Projektion auf die xz-Ebene in der $\mathrm{Cu}_{50} \mathrm{Ti}_{50}$ Probe bei $T=450 \mathrm{~K}$ für a.) $\mathrm{Cu}$ und b.) Ti. . . . . . . . . . . . . . . . . . . . . . . . 75

6.9 Trajektorien für a.) Atom 1 und b.) Atom 2. . . . . . . . . . . . . . . . . 76

6.10 a.) Varianz als Funktion der Zeit für Atom 1 und Atom 2, b.) NachbarKorrelationsfunktion für Atom 1 und Atom 2 für die Zeitpunkte $t_{1}=0$ und $t_{2}=t \ldots \ldots \ldots \ldots \ldots \ldots \ldots$

6.11 Potentielle Energie und Gesamtenergie von Atom 1 im Verlauf der Simulation. . . . . . . . . . . . . . . . . . . .

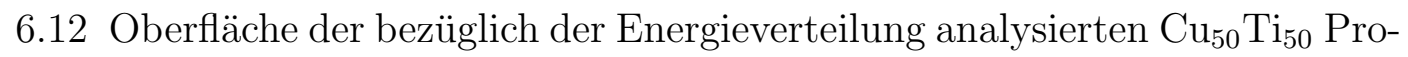
be; braun: $\mathrm{Cu}$ Atome, grau: Ti Atome . . . . . . . . . . . . . . . . . .

6.13 a.) Volumen und b.) Energie jeweils in Abhängigkeit der Temperatur beim Abkühlen (mit linearen Fits für die Energie) (Rate: $\dot{T}=-50 \mathrm{~K} / \mathrm{ps}$ ). . . .

$\underline{6.14}$ Paarkorrelationsfunktion der Si Bulk-Proben bei $T=0 \mathrm{~K}, T=1400 \mathrm{~K}$, $T=1400 \mathrm{~K}$ mit zusätzlicher Relaxation und bei $T=2200 \mathrm{~K} . \quad \ldots .$.

6.15 Paarkorrelationsfunktion der relaxierten Si Proben bei $T=1400 \mathrm{~K}$ als Bulk-Probe und mit zwei Oberflächen, die für einen Zeitraum von 300 ps relaxiert sind. . . . . . . . . . . . . . . . . . . 
6.16 Tiefenprofil des Diffusionskoeffizienten der relaxierten Si Probe bei $T=1000 \mathrm{~K}$, $T=1400 \mathrm{~K}$ und $T=1700 \mathrm{~K}$ jeweils in einem Zeitintervall von $\Delta t=500 \mathrm{ps}$ mit zwei Oberflächen in z-Richtung. . . . . . . . . . . . . . . . . . . . . 90

6.17 Histogramme der Verschiebungsweiten nach jeweils $\Delta t=500$ ps für dieselben Proben wie in Abb. 6.16 bei a.) $T=1000 \mathrm{~K}$, b.) $T=1400 \mathrm{~K}$, c.) $T=1700 \mathrm{~K}$. Die Linien entsprechen einem Fit nach Gleichung (6.8). .

6.18 Histogramme der Verschiebungsweiten bei $T=1400 \mathrm{~K}$ a.) für einen Zeitraum $\Delta t=1700 \mathrm{ps}$ in verschiedenen Bereichen der Probe und b.) für einen Oberflächenbereich als Vergleich zwischen $\Delta t=500$ ps und $\Delta t=1700$ ps. 92

6.19 Histogramme der Verschiebungsweiten bei $T=1000 \mathrm{~K}$ a.) für einen Zeitraum $\Delta t=8500 \mathrm{ps}$ in verschiedenen Bereichen der Probe und b.) für einen Oberflächenbereich als Vergleich zwischen $\Delta t=500$ ps und $\Delta t=8500$ ps. 93

6.20 a.) Histogramm der potentiellen Energien der Atome in der Si Schicht bei $T=1400 \mathrm{~K}$ zum Zeitpunkt nach der Untersuchung aus Abb. 6.18, b.) Position der Atome mit $E_{\text {pot }}>\mu+2 \sigma$ als Projektion auf die xz-Ebene. 94

6.21 Kantenlänge $L$ der Simulationsbox in den drei Raumrichtungen während des Heizprozesses einer zu Beginn amorphen Si Probe mit angelegter Zugspannung von $p=5$ kbar in x-Richtung. . . . . . . . . . . . . . . . 97

7.1 a.) Anzahl flüssiger Atome als Funktion der Zeit (Die roten Linien markieren zeitlich den Anfangs- und Endpunkt des flüssigen Bereichs.) und b.) räumliche Anordnung der flüssigen Atome zum Zeitpunkt $t=824 \mathrm{fs}$, jeweils für einen $\mathrm{Cu}$ Einschuss der Energie $E=1 \mathrm{keV}$ auf $\mathrm{CuTi}$ bei $T=10$ K Umgebungstemperatur. . . . . . . . . . . . . . . . . . . . . 105

7.2 a.) Anzahl flüssiger Atome und b.) Lebensdauer flüssiger Bereiche jeweils als Funktion der Einschussenergie nach einem Einschuss eines $\mathrm{Cu}$ Atoms auf eine CuTi Schicht bei einer Umgebungstemperatur von $\mathrm{T}=10 \mathrm{~K}$. Gestrichelte Linien entsprechen einem Spline-Fit der Daten. . . . . . . . 106

7.3 a.) Anzahl flüssiger Atome als Funktion der Zeit (Die roten Linien markieren zeitlich den Anfangs- und Endpunkt des flüssigen Bereichs.) und b.) räumliche Anordnung der flüssigen Atome zum Zeitpunkt $t=1501 \mathrm{fs}$, jeweils für einen $\mathrm{Cu}$ Einschuss der Energie $E=1 \mathrm{keV}$ auf $\mathrm{CuTi}$ bei $T=300$ K Umgebungstemperatur. . . . . . . . . . . . . . . . . . . 107

7.4 Verteilung der Verschiebungsweiten der flüssigen $\mathrm{Cu}$ Atome im Bereich der mit $\mathrm{Cu}$ Atomen der Energie $E=1 \mathrm{keV}$ beschossenen Oberfläche $(z>35 \AA)$ im Zeitraum $t_{1}=1$ ps bis $t_{2}=2$ ps bei einer Umgebungstemperatur von $T=10 \mathrm{~K}$ zusammen mit einem Fit entsprechend Gleichung (6.8). . . . . . . . . . . . . . . . . . . . . . . . . . . . . . . 108

7.5 Verteilung der Verschiebungsweiten bei thermisch angeregter und bei strahlungsinduzierter Dynamik für Cu Atome im Oberflächenbereich aus Abb. 6.6.a bzw. Abb. 7.4 jeweils mit dem zugehörigen Fit gemäß Gleichung (6.8). . . 109

7.6 Anzahl flüssiger Atome als Funktion der Zeit für einen $\mathrm{Cu}$ Einschuss der Energie $E=1 \mathrm{keV}$ auf CuTi bei $T=300 \mathrm{~K}$ Umgebungstemperatur (aus Abb. 7.3) mit Kennzeichnung der verschiedenen Abschnitte. . . . . . . . 111 
7.7 Varianz der $\mathrm{Cu}$ und Ti Atome im Oberflächenbereich nach Einschuss eines $\mathrm{Cu}$ Atoms der Energie $E=1 \mathrm{keV}$ und Anzahl der Adatome nach geometrischem Kriterium als Mittelung über mehrere Einschüsse jeweils bei einer Umgebungstemperatur von $T=300 \mathrm{~K}$. . . . . . . . . . . . . . 117

7.8 Verteilung der potentiellen Energie der $\mathrm{Cu}$ Atome vor $(t=0 \mathrm{ps})$ und nach $(t=50 \mathrm{ps})$ dem Einschuss eines $\mathrm{Cu}$ Atoms der Energie $E=1 \mathrm{keV}$. Teil a.) zeigt den gesamten Energiebereich und Teil b.) den Ausschnitt der Energie, für den Adatome in Betracht kommen. . . . . . . . . . . . . . . 119

7.9 Schräger Beschuss in der Simulation und Beschuss von Hügelflanken im realen Fall. . . . . . . . . . . . . . . . . . . . . . . . . . . . 120

7.10 Summe der atomaren Verschiebungen $\delta$ im Bereich der oberen $8 \AA$ für sukzessiven Beschuss einer CuTi Oberfläche unter einem Winkel von $\alpha=45^{\circ}$ bei $T=300 \mathrm{~K}$.

7.11 Doppeltlogarithmische Darstellung der Lebensdauern flüssiger Bereiche als Funktion der Einschussenergie für CuTi Schichten bei $T=10 \mathrm{~K}$ (aus Abb. 7.2) . . . . . . . . . . . . . . . . .

7.12 a.) Zeitliche Entwicklung der Anzahl flüssiger Atome (Die roten Linien markieren zeitlich den Anfangs- und Endpunkt des flüssigen Bereichs.) und b.) räumliche Anordnung der flüssigen Atome zum Zeitpunkt $t=246$ fs jeweils für denselben Einschuss der Energie $E=1 \mathrm{keV}$ bei einer Umgebungstemperatur von $T=10 \mathrm{~K} \ldots \ldots \ldots \ldots$

8.1 a.) Topographie der gemäß $\left[\mathrm{RML}^{+} 00\right]$ simulierten gewachsenen $480 \mathrm{~nm}$ dicken Schicht, b.) STM-Aufnahme der unbestrahlten $480 \mathrm{~nm}$ dicken Schicht aus Abb. 5.1 (Bildgröße: $L=200 \mathrm{~nm}$, Einheit der Höhenskala: nm). . . .

8.2 Topographie der gemäß Gleichung (8.1) simulierten Bestrahlung für Zeiten von a.) $5 \mathrm{~s}$ und b.) $1000 \mathrm{~s}$ (Bildgröße: $L=200 \mathrm{~nm}$, Einheit der Höhenskala: nm). . . . . . . . . . . . . . . . .

8.3 a.) Entwicklung der rms-Rauigkeit als Funktion der Bestrahlungszeit und b.) spektrale Leistungsdichten nach verschiedenen Bestrahlungszeiten; jeweils für das Modell aus Kap. 8.1. . . . . . . . . . . . . . . .

8.4 Entwicklung der rms-Rauigkeit als Funktion der Bestrahlungszeit für zwei verschiedene Rauschstärken im Modell aus Kap. 8.2 (Schwarze und rote Symbole liegen nahezu übereinander.). . . . . . . . . . . . . . . . 150

8.5 Spektrale Leistungsdichten nach verschiedenen Bestrahlungszeiten im Modell aus Kap. 8.2 für a.) $R=8.60 \cdot 10^{-6} \mathrm{~nm}^{4} / \mathrm{s}$ und b.) $R=5.38 \cdot 10^{-5} \mathrm{~nm}^{4} / \mathrm{s} .151$

8.6 Entwicklung der Oberflächentopographie als Funktion der Bestrahlungszeit für $R=5.38 \cdot 10^{-5} \mathrm{~nm}^{4} / \mathrm{s}$ im Modell aus Kap. 8.2 (Bildgröße: $L=200 \mathrm{~nm}$, Einheit der Höhenskala: nm). . . . . . . . . . . . . . . . . . . . . 152

8.7 a.) Entwicklung der rms-Rauigkeit als Funktion der Bestrahlungszeit und b.) spektrale Leistungsdichten jeweils mit verschiedenen lokalen Mittelungen, jeweils nach Kap. 8.3. . . . . . . . . . . . . . . . . . . . . . . . 154 
8.8 Entwicklung der rms-Rauigkeit als Funktion der Bestrahlungszeit für einen Glättungsterm der Form $|\nabla h|$. Bis $t=20$ s liegen schwarze und rote Symbole übereinander. . . . . . . . . . . . . . . . . . . . 156

8.9 Oberflächentopographien der Ausgangsstruktur sowie im Zeitbereich des Rauigkeitsminimums für die Simulation eines Glättungsterms der Form $|\nabla h|$ (Bildgröße: $L=200 \mathrm{~nm}$, Einheit der Höhenskala: nm). . . . . . . . 157

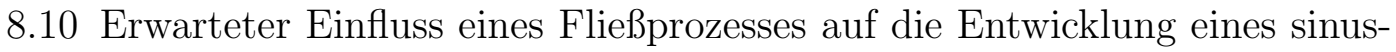
förmigen Höhenprofils. . . . . . . . . . . . . . . . . . . . . . . . . . . . . 158

8.11 a.) Entwicklung der rms-Rauigkeit als Funktion der Bestrahlungszeit und b.) spektrale Leistungsdichten für $\tilde{c}_{1}=-10 \mathrm{~s}^{-1}$, jeweils nach Gleichung (8.8).159

8.12 Topographie der gemäß Gleichung (8.8) simulierten Bestrahlung mit $\tilde{c}_{1}=$ $10 \mathrm{~s}^{-1}$ für Zeiten von a.) $5 \mathrm{~s}$ und b.) $50 \mathrm{~s}$ (Bildgröße: $L=200 \mathrm{~nm}$, Einheit der Höhenskala: nm). . . . . . . . . . . . . . . . . . . . . . 160

8.13 a.) Entwicklung der rms-Rauigkeit als Funktion der Bestrahlungszeit und b.) spektrale Leistungsdichten für die Simulation des Modells aus Gleichung $(8.9)$. . . . . . . . . . . . . . . . . . . . . . . . . . 161

9.1 Vergleich der Rauigkeitsentwicklung im Experiment und im Kontinuumsmodell gemäß Kap. 8.2 mit $R=5.38 \cdot 10^{-5} \mathrm{~nm}^{4} / \mathrm{s}$. Für Daten des Kontinuumsmodells wird in a.) der Zeitparameter der Simulation und in b.) ein umskalierter Zeitparameter verwendet. Die experimentellen Daten sind als Funktion der Bestrahlungszeit aufgetragen. . . . . . . . . . . . . . . 167 
Abbildungsverzeichnis 


\section{Einleitung}

Die Untersuchung von Oberflächenstrukturen bildet ein weites Feld aktueller Forschung. Auch im Hinblick auf die fortschreitende Miniaturisierung elektronischer Bauteile ist es für die Anwendung wichtig, die Struktur von Grenz- bzw. Oberflächen zu kontrollieren und je nach Anforderung herzustellen. Um dazu in der Lage zu sein, ist es von fundamentaler Bedeutung, die Mechanismen, die zur Bildung einer Struktur beitragen, zu identifizieren.

Für die Identifikation der wesentlichen Mechanismen bieten sich amorphe Systeme an, die wegen der vorhandenen räumlichen Isotropie keine strukturell induzierte Richtungsabhängigkeit der Oberflächenstrukturen erwarten lassen und somit das einfachst mögliche Modellsystem bilden. Außerdem finden amorphe dünne Schichten in vielen technischen Bereichen ihre Anwendung. Metallische amorphe Schichten lassen sich durch Kondensation aus der Gasphase bei sehr tiefen Temperaturen (auf Helium-gekühlten Substraten) herstellen, wie es für einkomponentige Systeme bereits seit den Arbeiten von Buckel und Hilsch bekannt ist [BH54]. Mehrkomponentige glasbildende Systeme lassen sich auch bei Raumtemperatur durch Kokondensation als amorphe Schicht herstellen. Ein Vetreter solcher glasbildenden metallischen Legierungen ist $\mathrm{Zr}_{65} \mathrm{Al}_{7.5} \mathrm{Cu}_{27.5}$, das bezüglich der Strukturbildung während des Wachstums ausführlich untersucht worden ist [RMS97; MMS99; $\mathrm{RML}^{+}$00; SSM02; VSMS03].

Strukturentwicklung findet dann statt, wenn die Oberfläche einem dynamischen Prozess unterliegt. Ein solcher Prozess ist beispielsweise das erwähnte Wachstum einer dünnen Schicht, aber auch die Bestrahlung einer Oberflächenstruktur stellt einen dynamischen Prozess dar, der für die Entwicklung der Oberflächenstruktur verantwortlich sein kann. Im Rahmen dieser Arbeit ist mit dem Begriff der Bestrahlung stets der Beschuss einer Oberfläche mit Ionen gemeint. Die Begriffe Ionenbestrahlung und Ionenbeschuss werden im Folgenden synonym verwendet. Der Einfluss des Ionenbeschusses wird hier untersucht. Durch Ionenbeschuss getriebene Strukturbildungsphänomene sind seit langer Zeit bekannt und besonders im Energiebereich weniger keV untersucht worden. Dieser Energiebereich ist auch bezüglich der technologischen Anwendung interessant, weil sich Ionen dieser Energie relativ leicht erzeugen lassen. Deshalb wird Ionenbeschuss dieser Energie in vielen Bereichen seit langem verwendet, beispielsweise auch, um Oberflächenschichten abzutragen. Durch die Ionenbestrahlung können sich Wellenstrukturen bilden, wenn die Ionen schräg zur Substratnormalen eingeschossen werden [NSC62]. Beim Beschuss entlang der Substratnormalen oder auch bei schrägem Einschuss und gleichzeitiger Rotation des Substrats mit der Substratnormalen als Drehachse wird ebenfalls unter verschiedenen Bedingungen Strukturbildung beobachtet [FDK ${ }^{+}$99; FSB00], wobei diese Strukturen in der Oberfläche isotrop sind. Die Entwicklung einer regelmäßigen Struktur beruht in der Regel auf einem Zusammenspiel von glättenden und aufrauenden 


\section{Einleitung}

Prozessen, wie es im Modell von Bradley und Harper beschrieben wird [BH88]. Jedoch ist bisher der mikroskopische Ursprung der glättenden Mechanismen nicht geklärt. Es werden in der Literatur für den Energiebereich weniger keV verschiedene Prozesse diskutiert, die zur Glättung beitragen. Dies sind Oberflächendiffusion [BH88; EAC ${ }^{+}$99], Fließprozesse [UHC01] sowie Glättung durch ballistischen Transport [CV96; $\mathrm{MGC}^{+} 05$ ]. Ziel dieser Arbeit ist es, herauszufinden, welche Prozesse dominieren und welche weniger wichtig sind.

Für das metallische Glas $\mathrm{Zr}_{65} \mathrm{Al}_{7.5} \mathrm{Cu}_{27.5}$ ist bekannt, dass sich durch Ionenbestrahlung im niedrigen $\mathrm{MeV}$ Energiebereich eine strukturierte Oberfläche glätten lässt [MA01]. Für diesen Energiebereich konnte außerdem ein Volumenfließen als dominierender Glättungsmechanismus identifiziert werden. Im Energiebereich weniger keV sind andere Glättungsmechanismen zu erwarten, weil sich das Verhältnis zwischen der Eindringtiefe der Ionen und der typischen Strukturgröße auf der bestrahlten Oberfläche in Abhängigkeit von der Bestrahlungsenergie ändert. Hier ist nun das Ziel, für Bestrahlungsenergien der Größenordnung einiger keV die Auswirkung der Ionenbestrahlung an diesem metallischen Glas zu untersuchen und die relevanten Prozesse zu identifizieren.

Die Phänomene der Strukturentwicklung durch die Ionenbestrahlung werden experimentell untersucht, so dass der Effekt der Bestrahlung auf mesoskopischer Skala deutlich wird. Prinzipiell kann die Entwicklung von Oberflächenstrukturen im Nichtgleichgewichtszustand durch Kontinuumsmodelle in Form von stochastischen Ratengleichungen beschrieben werden [BS95]. Ihren Ursprung haben solche Modelle in Arbeiten von Mullins und Herring zur Analyse kinetischer Prozesse [Her50; Mul57; Mul59]. Die Analyse der experimentellen Ergebnisse im Rahmen solcher Modelle lässt Rückschlüsse auf die beteiligten Prozesse zu. Um die mikroskopische Ursache der Strukturentwicklung genauer zu untersuchen, werden klassische Molekulardynamik(MD)-Simulationen durchgeführt und in Verbindung mit Kontinuumsmodellen, die die mesoskopische Skala beschreiben, ausgewertet [VM07]. Um die relevanten Prozesse für eine Vielzahl von Materialsystemen aufklären zu können, werden in den MD-Simulationen zwei Vertreter verschiedener Klassen von amorphen Systemen untersucht. Das ist zum einen das fragile metallische Glas CuTi und zum anderen das starke Glas Si.

In Verbindung mit den Erkenntnissen aus den durchgeführten Experimenten und den MD-Simulationen werden stochastische Ratengleichungen, die die Strukturbildung auf mesoskopischer Skala beschreiben, entwickelt und numerisch gelöst.

Neben der Untersuchung von Bestrahlungsphänomenen werden zum Vergleich auch MD-Simulationen zu thermisch angeregten Prozessen durchgeführt. Hier werden dieselben Materialien untersucht wie bei den Untersuchungen zur Bestrahlung. Der Schwerpunkt liegt hier auf der Oberflächendynamik und ermöglicht einen Vergleich zwischen thermisch angeregten und strahlungsinduzierten Prozessen jeweils in identischen Materialien und damit eine Abgrenzung zwischen den unterschiedlichen Arten der Anregung. Für die Untersuchungen zu thermischen Prozessen an CuTi-Oberflächen [VM05] sind Vergleiche mit und Interpretationen von früheren experimentellen Untersuchungen am selben System [GHK98] möglich.

Das generelle Ziel der vorliegenden Arbeit ist, einen Beitrag zur Entschlüsselung der atomaren Kinetik an Oberflächen im angeregten Zustand zu leisten und das Verständnis 
des Zusammenhangs der atomaren Prozesse mit Strukturbildungsphänomenen auf gröberer Skala (Größenordung: einige $\mathrm{nm}$ ) voran zu treiben.

Strukturiert ist die Arbeit wie folgt. Zunächst werden die Methoden vorgestellt. Das sind die experimentellen Methoden, die Methoden der MD-Simulation und die der stochastischen Ratengleichungen (Kap. 2-4). Es folgen die Kapitel mit den Ergebnissen. Begonnen wird mit den experimentellen Ergebnissen (Kap. 5), um die wesentlichen Phänomene der Bestrahlungseffekte zu zeigen. Darauf folgen die MD-Simulationen, wobei das Kapitel über thermische Untersuchungen hier eingeschoben wird (Kap. 6), um später den Vergleich mit den anschließend vorgestellten strahlungsinduzierten Prozessen (Kap. 7) zu ermöglichen. Danach werden die Ergebnisse aus Simulationen von stochastischen Ratengleichungen gezeigt, in die die Erkenntnisse aus den vorangegangenen Kapiteln bereits eingehen (Kap. 8). Weil die verschiedenen Kapitel aufeinander aufbauen, werden die Ergebnisse bereits im jeweiligen Kapitel diskutiert. Eine zusammenfassende Diskussion der wesentlichen Ergebnisse, die insbesondere die verschiedenen Aspekte der Arbeit verbindet, folgt in Kap. 9, bevor eine Zusammenfassung mit einem Ausblick den Abschluss bildet. 


\section{Experimentelles}

Im experimentellen Teil dieser Arbeit sollen dünne Schichten metallischer Gläser hergestellt werden und anschließend durch Ionenbestrahlung modifiziert werden. Dabei steht jeweils die sich durch die Behandlung ändernde Oberflächenstruktur im Mittelpunkt des Interesses.

Zunächst soll auf die Herstellungs- und Analysemethoden eingegangen werden. Der wesentliche Teil der experimentellen Prozesse, nämlich die Herstellung und Bestrahlung der Proben sowie die Analyse der Oberflächenstruktur, findet im Ultrahochvakuum (UHV) statt. Daher soll die entsprechende UHV-Anlage beschrieben werden, in der diese Arbeitsschritte durchgeführt werden. Nach einer kurzen Beschreibung des experimentell verwendeten Systems wird die Probenherstellung durch Elektronenstrahlverdampfen erläutert. Des Weiteren soll beschrieben werden, wie die hergestellten Proben der Bestrahlung durch Ionen unterzogen werden, um die Oberflächenstruktur zu verändern. Als wesentliche Analysemethode dient die Untersuchung mit einem Rastertunnelmikroskop (STM $\left.{ }^{1}\right)$ zur Charakterisierung der Oberflächenmorphologie. Zur Schichtdickenbestimmung wird Kleinwinkelröntgenreflektometrie eingesetzt, während die Struktur der Proben mittels Weitwinkelröntgendiffraktometrie untersucht wird.

\subsection{Die Ultrahochvakuum-Anlage}

Die verwendete UHV-Anlage, die in Abb. 2.1 skizziert ist, besteht aus drei Kammern. In der "Aufdampfkammer" findet mit den drei Elektronenstrahlverdampfern die Herstellung der Schichten statt, in der "Analysenkammer" können die Schichten durch Ionenbestrahlung modifiziert und ihre Oberflächenstruktur mittels Rastertunnelmikroskopie untersucht werden und die dritte Kammer dient als Schleuse. Die Aufdampfkammer und die Analysenkammer sind jeweils durch ein Plattenventil mit der Schleusenkammer verbunden, so dass ein Transfer der Proben zwischen den verschiedenen Kammern über magnetisch gekoppelte Transferstangen möglich ist ohne das Vakuum zu verlassen. In den Kammern, die zur Herstellung und Untersuchung der Proben verwendet werden, wird typischerweise ein Druck von weniger als $5 \cdot 10^{-10}$ mbar erreicht. Dieser Druck ergibt sich durch Verwendung einer Turbomolekularpumpe mit vorgeschalteter Rotationspumpe an der Schleusenkammer, in der der Druck höher ist, sowie Ionengetterpumpen und Titansublimationspumpen in der Aufdampf- und Analysenkammer. Um mit diesen Pumpen den angegebenen Druck zu erreichen, ist außerdem noch ein Ausheizvorgang nötig. In verschiedenen früheren Arbeiten wurde diese UHV-Anlage bereits benutzt und ist somit auch vielfach beschrieben [Rei97; May97; May00; Vau02; Str04].

\footnotetext{
${ }^{1}$ Englisch: scanning tunneling microscope
} 


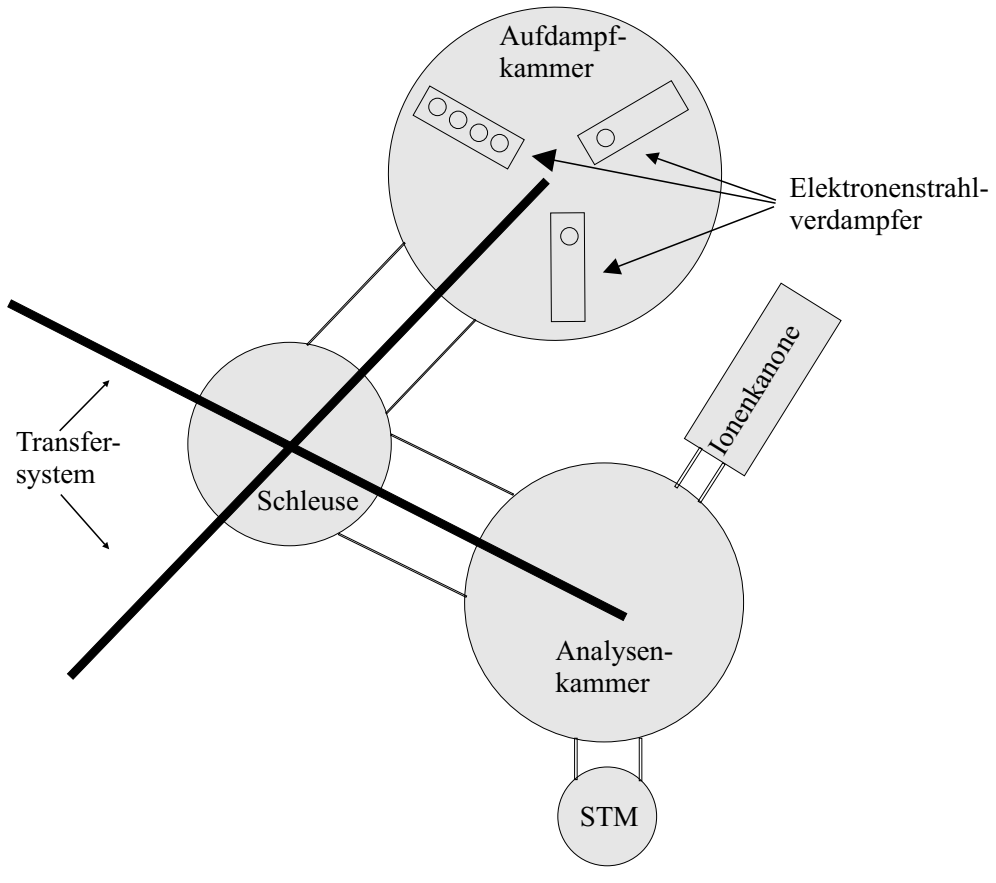

Abbildung 2.1: Schematische Darstellung der UHV-Anlage.

Im Rahmen dieser Arbeit wurde zunächst eine Ionenkanone des Typs „Ion Bombardment Gun, Model 981-2043“2, die bereits in die Analysenkammer integriert war, neu positioniert, um den Ionenbeschuss von Oberflächen entlang der Oberflächennormalen zu ermöglichen. Das benötigte Edelgas kann über ein fein regulierbares Ventil in kleinen Mengen in die Analysenkammer eingelassen werden. Zu einem späteren Zeitpunkt wurde diese Kanone ersetzt durch eine des Typs „Ion Source IQE 12/38“3, in der man das nötige Edelgas gezielt in die Kanone einlässt und es mit einer zusätzlich eingebauten Turbomolekularpume differentiell abpumpt, so dass weit weniger Edelgas in die Analysenkammer eindringt. Diese Modifikation war nötig, weil außerdem die Möglichkeit geschaffen wurde, eine zusätzliche Hochspannung zwischen dem Probenhalter und der Ionenkanone anzulegen, damit auch höhere Bestrahlungsenergien als die von der Kanone vorgegebenen möglich sind. Wenn nämlich ein Druck von ca. $10^{-5}$ mbar, wie er in der Ionenkanone nötig ist, in der Analysenkammer herrscht, würden solche Hochspannungen (einige $\mathrm{kV}$ ) zu Überschlägen führen, so dass ein deutlich niedrigerer Druck, wie er bei einer differentiell gepumpten Ionenkanone möglich ist, erforderlich ist. Weitere Details zu den verwendeten Ionenkanonen sind in Kap. 2.2.3 beschrieben.

\footnotetext{
${ }^{2}$ Firma Varian

${ }^{3}$ Firma SPECS
} 


\subsection{Probenherstellung und Eigenschaften}

\subsubsection{Materialeigenschaften}

Um die zu untersuchenden Prozesse auch möglichst exakt modellieren zu können, ist es am einfachsten, ein isotropes Materialsystem zu betrachten. Dafür bietet sich ein amorphes System an, in dem keine kristallinen Vorzugsrichtungen bestehen. In dieser Arbeit wird das metallische Glas $\mathrm{Zr}_{65} \mathrm{Al}_{7.5} \mathrm{Cu}_{27.5}$ verwendet, weil für dieses außerdem das Schichtwachstum in den vergangenen Jahren bereits sehr genau untersucht wurde [RMS97; MMS99; RML ${ }^{+} 00 ;$ SSM02; VSMS03] und somit die entsprechenden Ergebnisse hier verwendet werden können. Die Zusammensetzung ist dabei so optimiert, dass der Temperaturbereich der unterkühlten Schmelze (Differenz zwischen Kristallisationsund Glastemperatur) maximal ist [IZM92; IKT ${ }^{+}$94]. Wegen unterdrückter Keimbildung ist das System sehr stabil gegen Kristallisation. Diese hohe Stabilität gegenüber Kristallisation und die damit verbundene gute experimentelle Handhabbarkeit ist neben der bereits erwähnten Kenntnis der Wachstumseigenschaften ein weiterer Grund für die Wahl dieses Systems. Der Temperaturbereich der unterkühlten Schmelze wird bei Abweichungen von der idealen Zusammensetzung kleiner, wie man es im ternären Phasendigramm erkennen kann (siehe Abb. 2.2). Für kleine Abweichungen in der Zusammensetzung, die bei der Schichtherstellung (siehe Kap. 2.2.2) durchaus möglich sind, bleibt der Temperaturbereich der unterkühlten Schmelze groß genug.

Es sollte außerdem erwähnt werden, dass auch im amorphen Festkörper eine Nahordnung existiert. Somit ist auf sehr kleiner Skala die Isotropie leicht gestört, wenngleich die Gesamtstruktur immernoch als ungeordnet angesehen werden muss. Als ein Charakterisierungsmerkmal eines Glases dient der Fragilitätsindex, der die Abweichung der Entwicklung der Viskosität als Funktion der Temperatur von einem Arrhenius-Verhalten quantifiziert [El190], und über den sich Gläser in starke und fragile einordnen lassen. Dabei sind die starken Gläser die mit wenig und die fragilen Gläser die mit deutlicher Abweichung vom Arrhenius-Verhalten. Dieser Fragilitätsindex wurde von M. Weiß zu $m=38.4$ bestimmt, so dass $\mathrm{Zr}_{65} \mathrm{Al}_{7.5} \mathrm{Cu}_{27.5}$ zwischen den Extrembeispielen für fragile $\left(m \approx 190\right.$ für Orthoterphenyl) und starke $\left(m \approx 16\right.$ für Netzwerkglas $\left.\mathrm{SiO}_{2}\right)$ Gläser steht [Wei98].

Die Herstellung eines Glases kann durch rasches Abschrecken erfolgen, wie es sich für die Herstellung von Bulkproben anbietet. Dünne glasartige Filme geeigneter Legierungen lassen sich durch Kondensation der Bestandteile aus der Dampfphase auf einem bei Raumtemperatur befindlichen Substrat herstellen [Zar91]. So verhält es sich auch hier, so dass in dieser Arbeit das Aufdampfen die Herstellungsmethode ist, wie es im folgen Kapitel beschrieben wird. Dabei kann man annehmen, dass die auftreffenden Atome in einem metastabilen Gleichgewicht auf dem relativ kalten Substrat nach einer kurzen diffusiven Bewegung „einfrieren“ [El190]. Da sie unkorreliert auftreffen, kann sich also keine Fernordnung herausbilden. 


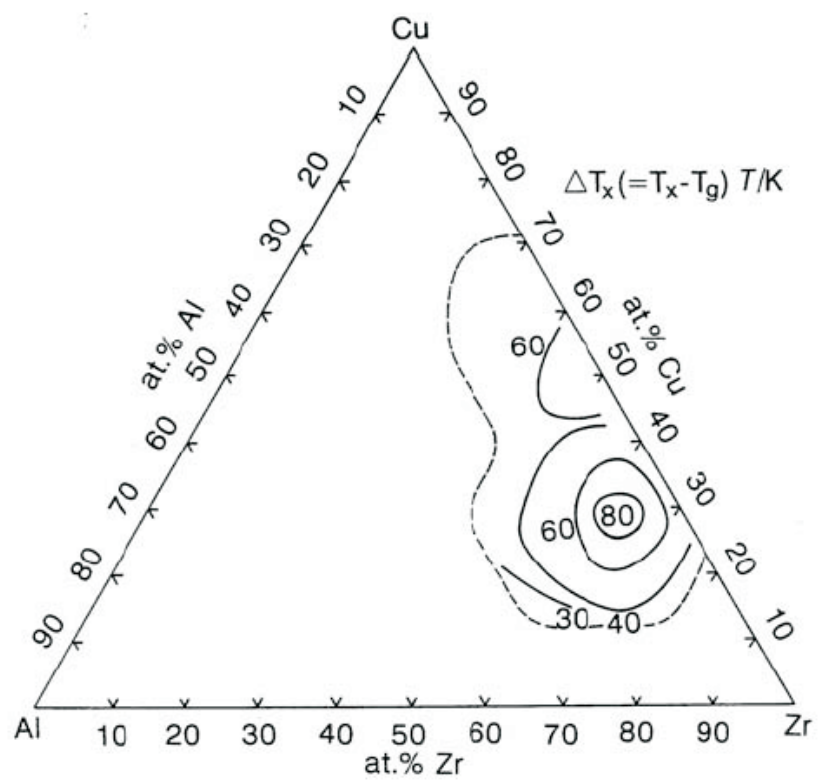

Abbildung 2.2: Temperaturintervall $\Delta T_{x}$ der unterkühlten Schmelze im ternären Phasendiagramm von $\mathrm{ZrAlCu}$ (nach [IZM92]; die gestrichelte Linie begrenzt den Bereich der amorphen Phase).

\subsubsection{Schichtherstellung}

In dieser Arbeit wurden dünne Schichten des metallischen Glases $\mathrm{Zr}_{65} \mathrm{Al}_{7.5} \mathrm{Cu}_{27.5}$ durch Kokondensation aus der Gasphase hergestellt. Als Ausgangsmaterial für diese Schichten dienen Reinstmaterialien, die in Tab. 2.1 genauer spezifiziert sind, und als Substrate werden thermisch oxidierte Silizium(100)-Wafer, bzw. daraus passend zugeschnittene Teile, verwendet. Die Ausgangsmaterialien werden in die wassergekühlten Kupferbzw. Molybdäntiegel der drei Elektronenstrahlverdampfer 4 eingebaut, wo sie mit einem in den Tiegel gelenkten Elektronenstrahl geheizt und aufgeschmolzen werden. Vor der ersten Schichtherstellung nach neuem Materialeinbau wird jedes Material zunächst aufgeschmolzen, um Verunreinigungen ausgasen zu lassen und das Material in eine gleichmäßige Form zu bringen. Während der Schichtherstellung wird die Aufdampfrate durch Schwingquarzwaagen über jedem einzelnen Verdampfer und eine jeweilige Eichmessung, die die geometrischen Unterschiede zwischen Schwingquarzwaage und Substrathalter charakterisiert, gemessen. Dabei beträgt die Genauigkeit der Schwingquarzwaagen $0.01 \mathrm{~nm} / \mathrm{s}$. Es können also drei verschiedene Materialien gleichzeitig mit definierter Rate verdampft werden, so dass man ternäre Legierungen mit gewünschter Zusammensetzung herstellen kann. Die Umrechnung zwischen der Zusammensetzung in Atomprozent und den beim Aufdampfen relevanten Raten ist in [Rei97] beschrieben. Vor dem Substrat befindet sich ein Shutter, der die Probe abschatten oder sie dem Teilchenstrom aussetzen kann. Sobald alle Materialien mit konstanter Rate verdampfen, wird dieser

${ }^{4}$ ESV4 Verdampfer, Firma Leybold 


\begin{tabular}{|l||l|l|}
\hline Element & Reinheit & Hersteller \\
\hline \hline $\mathrm{Zr}$ & $99.8 \%$ & Strem Chemicals \\
\hline $\mathrm{Al}$ & $99.999 \%$ & Heraeus GmbH \\
\hline $\mathrm{Cu}$ & $99.999 \%$ & Johnson Matthey (Alfa Products) \\
\hline
\end{tabular}

Tabelle 2.1: Reinstmaterialien.

Shutter geöffnet und die gewünschte Legierung wächst auf dem Substrat.

\subsubsection{Ionenbestrahlung}

Sobald eine Schicht hergestellt ist, kann sie in die Analysenkammer transferiert werden, ohne das Vakuum zu brechen. Dort wird die Probe dann so justiert, dass sie im Folgenden unter senkrechtem Einfall mit Ionen bestrahlt wird.

Wie zuvor beschrieben kamen zwei verschiedene Ionenkanonen zum Einsatz. Die prinzipielle Arbeitsweise ist aber bei beiden Geräten gleich. Zunächst wird ein Wolframfilament durch elektrischen Strom geheizt, so dass Elektronen ausgelöst werden. Diese werden durch Anlegen einer Hochspannung beschleunigt, ionisieren die Edelgasatome und die Ionen können dann wiederum durch eine Hochspannung bis zur gewünschten Energie beschleunigt werden. Durch ein elektrooptisches Linsensystem kann der Ionenstrahl dann fokussiert werden, so dass ein Strahldurchmesser im Millimeterbereich auf der Probe erreichbar ist. Des Weiteren lassen sich oszillierende Ablenkspannungen in zwei zueinander senkrechte Richtungen, die beide senkrecht zum Strahl liegen, anlegen. Das führt dazu, dass ein größerer Bereich der Probe gleichmäßig bestrahlt wird.

Wichtige Charakterisierungsgrößen für die Bestrahlung sind neben der Energie noch der Fluss (Ionen/(Fläche.Zeit)) und die Fluenz (Ionen/Fläche). In der verwendeten UHV-Anlage ist es möglich, den Fluss mithilfe eines Faradaycups zu messen. In einem Faradaycup treffen die Ionen durch eine Öffnung bekannter Fläche auf eine Metallfolie (hier: Molybdän) und erzeugen einen elektrischen Strom. Weil die Oberfläche des umgebenden Zylinders („Cup“) sehr viel größer als die Fläche der Öffnung ist, verlässt nur ein sehr kleiner Anteil der durch die auftreffenden Ionen induzierten Sekundärelektronen den Faraday Cup, so dass sie kaum zum gemessenen Nettostrom beitragen. Daher wird insgesamt der tatsächliche, nicht durch ausgelöste Sekundärelektronen verfälschte, Strom gemessen. Als Fehlerquelle bleibt natürlich noch die Öffnung des Zylinders, durch die weiterhin Sekundärelektronen entweichen können. Da diese Öffnung aber sehr klein im Vergleich zum Zylinder ist, ist der durch diesen Effekt hervorgerufene Fehler klein. Bei Annahme einfach geladener Ionen kann dann der gemessene elektrische Strom in die Anzahl von Ionen umgerechnet werden. Die Fläche der Apertur des Faradaycups ist ebenfalls bekannt (500 $\mu \mathrm{m}$ Durchmesser) und somit kann der Fluss bestimmt werden. Da die Position des Faradycups in allen Raumrichtungen variabel ist, kann der Fluss an allen Stellen bestimmt werden, an denen sich bei der Bestrahlung die Probe befindet. Man kann also ein Strahlungsprofil für den interessierenden Bereich erstellen (siehe Abb. 2.3). Außerdem kann der Strom gemessen werden, der über die Probe abfließt, so dass eine Positionierung der Probe im Zentrum des Ionenstrahls immer möglich ist. So 

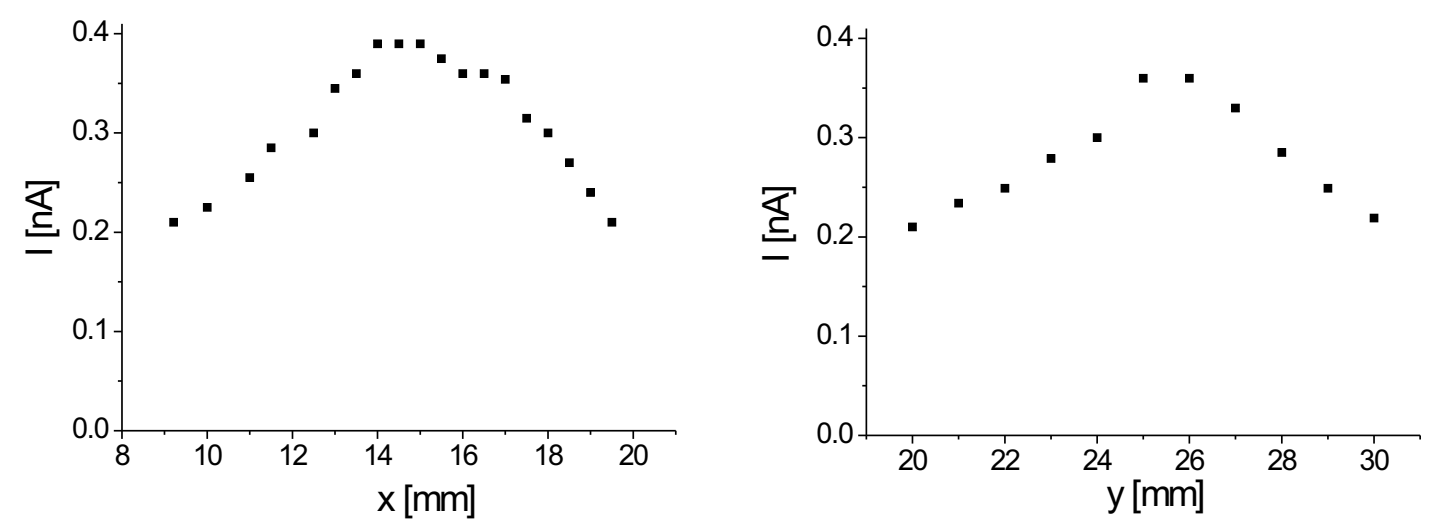

Abbildung 2.3: Ionenstrahlprofil der „Ion Bombardment Gun 981-2043“ entlang der Richtungen senkrecht zur Strahlrichtung.

ist gewährleistet, dass in dem Bereich der Probe, der später bezüglich der Morphologie mit dem STM untersucht wird, der relevante Fluss bekannt ist.

Zunächst wurde die Ionenkanone „Ion Bombardment Gun 981-2043“ der Firma Varian verwendet, mit der Bestrahlungsenergien von 0 bis $3 \mathrm{keV}$ möglich sind. Als Edelgas wurde Krypton gewählt. Es ist das Edelgas, das mit seiner Masse denen der Hauptbestandteile der zu bestrahlenden Schicht am nächsten ist $\left(m_{K r}=83.80 u, m_{Z r}=91.22 u, m_{C u}=\right.$ 63.55u). Da das Gas bei dieser Ionenkanone nicht differentiell abgepumpt werden kann, wird die Analysenkammer mit hochreinem Krypton auf ca. $10^{-5}$ mbar geflutet. Abb. 2.3 zeigt das zugehörige Strahlungsprofil für einen fokussierten Strahl, der ein Gebiet abrastert.

Im weiteren Verlauf der Arbeit wurde die beschriebene Ionenkanone durch eine des Typs „Ion Source IQE 12/38“ der Firma SPECS ersetzt. Zusammen mit dem Gaseinlass wurde eine Turbomolekularpumpe an die Kanone angebaut, so dass das Gas bereits im Bereich der Kanone differentiell abgepumpt werden kann. Dadurch bleibt die UHVKammer bei einem deutlich kleineren Druck (etwa $5 \cdot 10^{-8}$ mbar) als die Kanone. Mit dieser Kanone sind Bestrahlungen im Energiebereich von 0 bis $5 \mathrm{keV}$ möglich. Außerdem wurde die Möglichkeit geschaffen, zwischen Probenhalter und Ionenkanone eine Hochspannung bis ca. $10 \mathrm{kV}$ anzulegen, so dass damit Bestrahlungsenergien bis ca. 15 keV möglich sind. Auch mit dieser Kanone lässt sich ein fokussierter Strahl erzeugen, mit dem man eine Fläche wählbarer Größe abrastern kann. Dabei ist hier das Rastern gleichmäßiger und über einen größeren Bereich möglich als mit der zuvor beschriebenen Kanone. 


\subsection{Analysemethoden}

\subsubsection{Rastertunnelmikroskopie}

Die Rastertunnelmikroskopie hat sich seit dem Bau des ersten Gerätes dieser Art durch Binnig und Rohrer [BRGW82] zu einer Standarduntersuchungsmethode für Oberflächenmorphologien elektrisch leitender Proben entwickelt. Das Messprinzip beruht darauf, dass man eine metallische Spitze sehr dicht (ca. $1 \mathrm{~nm}$ ) über die zu untersuchende Oberfläche bringt und eine elektrische Spannung zwischen Spitze und Probe anlegt. Zwischen Spitze und Probe dient ein Luft- oder Vakuumspalt als Potentialbarriere, die aufgrund der Gesetze der Quantenmechanik von Elektronen durchtunnelt werden kann, so dass ein elektrischer Strom fließt und detektiert werden kann. Die Tunnelwahrscheinlichkeit $T$ ist dabei abgesehen von Konstanten durch die Breite der Barriere $d$ und die Austrittsarbeit für Elektronen aus dem Metall $\phi$ gemäß

$$
T \propto e^{-\frac{2 \sqrt{2 m_{e} \phi}}{\hbar} d}
$$

bestimmt [Bon93]. Dabei bezeichnet $m_{e}$ die Elektronenmasse und $\hbar$ das Plancksche Wirkungsquantum. Weiterhin ist der Strom dem Produkt aus angelegter Spannung und Tunnelwahrscheinlichkeit proportional, sofern das Produkt aus angelegter Spannung und Elementarladung nicht die Austrittsenergie der Elektronen aus dem Material übersteigt [HH91]. Diese Bedingung ist bei allen durchgeführten Messungen erfüllt. Für weitere Details sei an dieser Stelle auf die vorhandene ausführliche Literatur verwiesen (z.B. [HH91; Bon93; HT87]).

Wichtig ist, wie der gemessene Tunnelstrom mit den Eigenschaften der Probe zusammenhängt. In einem einfachen Modell, in dem die Spitze als punktförmig idealisierte Stromquelle angenommen wird, erhält man als Ergebnis, dass der Tunnelstrom proportional zur Elektronendichte der Probe ist [HT87; TH85]. Demnach resultiert das gemessene Stromsignal aus einer Überlagerung von Morphologie auf einer größeren (mesoskopischen) Skala und chemischer Struktur auf einer kleineren (atomaren) Skala. Welcher Einfluss dominiert, hängt von der Struktur der Oberfläche ab. Bei atomar glatten Oberflächen dominiert der Einfluss der chemischen Struktur, wohingegen bei rauen Oberflächen die Morphologie dominiert. Hier wird für alle Messungen der KonstantstromModus (constant current mode) benutzt, bei dem der Abstand zwischen Spitze und Probe so geregelt wird, dass der Strom konstant bleibt. Wenn man nun die Probe mit der Spitze abrastert, entspricht die relative Höhe der Spitze bei den in dieser Arbeit untersuchten relativ rauen (zumindest nicht atomar glatten) Oberflächen im Wesentlichen der Oberflächenmorphologie. Die feine laterale Rasterung sowie exakte Angaben über die Höhe der Spitze sind durch Verwendung von piezoelektrischen Kristallen zur Steuerung möglich. Bei allen rastertunnelmikroskopischen Untersuchungen muss stets bedacht werden, dass das gemessene Signal sich aus einer Faltung der Probeneigenschaften mit den Spitzeneigenschaften ergibt. Daher müssen die verwendeten Spitzen weitestgehend optimiert werden, damit die Eigenschaften der Probe die Ursache für das Messsignal sind. 
Für sämtliche Messungen wurde ein Omicron UHV-STM-1, das in die zuvor beschrieben UHV-Anlage integriert ist, verwendet. Die Spitzen und Proben lassen sich bei arretiertem Meßkopf in situ wechseln. Als Spitzenmaterial wird Wolfram verwendet. Die Spitzen werden durch nasschemisches Ätzen hergestellt, wie es sehr ausführlich in [Str04] beschrieben wird. Dabei wird der Wolframdraht (0.5 mm Durchmesser) senkrecht in 1 molare KOH-Lösung getaucht und dient als eine Elektrode. Der untere Teil des Drahtes wird elektrisch abgeschirmt, so dass durch Anlegen einer $6 \mathrm{~V}$ bzw. $12 \mathrm{~V}$ Wechselspannung über eine zweite Elektrode aus Edelstahl der Wolframdraht verstärkt an der Grenzfläche zwischen Lauge und Luft geätzt wird. Nach einiger Zeit reißt der Draht an dieser Stelle ab und für den in die Lauge fallenden Teil ist der Ätzprozess gestoppt, während der obere Teil weiter geätzt wird, bis der Flüssigkeitsfilm abreißt. Nach einer Reinigung in heißem Wasser wird der untere Teil als Tunnelspitze verwendet.

\subsubsection{Röntgendiffraktometrie und -reflektometrie}

Mittels Röntgendiffraktometrie können je nach Einfallswinkel Informationen über die Struktur einer Probe und bei dünnen Schichten außerdem über die Schichtdicke und die Rauigkeit der Grenzflächen gewonnen werden. Sämtliche Untersuchungen wurden am Röntgendiffraktometer D 5000 der Firma Siemens durchgeführt. Die Röntgenquelle liefert die mittels Einkristallmonochromator selektierte charakteristische $C u_{K_{\alpha}}$-Strahlung mit einer Wellenlänge von $\lambda_{C u_{K_{\alpha}}}=0.15418 \mathrm{~nm}$ als gewichtetetem Mittel aus den Wellenlängen der $C u_{K_{\alpha 1}}$ und $C u_{K_{\alpha 2}}$ Linien. Im Folgenden sei diese Wellenlänge abkürzend mit $\lambda$ gekennzeichnet. Gemessen wurde jeweils in Bragg-Brentano Geometrie, d.h. Quelle, Probe und Detektor sind in einer $\Theta / 2 \Theta$ Geometrie aufgebaut und die Intensität am Detektor wird als Funktion des Einfallswinkels $\Theta$ aufgenommen. In dieser Geometrie steht unter der Annahme elastischer Streuung der Streuvektor, der sich als Differenz von einfallender und gebeugter Röntgenstrahlung ergibt, senkrecht zur Schichtoberfläche. Daher können nur Informationen über Strukturen in dieser Richtung gewonnen werden.

\subsubsection{Kleinwinkelröntgenreflektometrie}

Röntgenreflektometrie bei kleinen Winkeln wurde in dieser Arbeit zur Bestimmung von Schichtdicken eingesetzt. Dabei nutzt man aus, dass die Röntgenstrahlung oberhalb des Winkels, bis zu dem Totalreflexion stattfindet, zum Teil eine Grenzfläche durchdringt und zum Teil reflektiert wird. Bei sehr kleinen Winkeln tritt Totalreflexion ein, weil für Röntgenstrahlung der Brechungsindex in Metallen etwas kleiner als eins ist. Der unterschiedliche Streukontrast verschiedener Schichten ergibt sich aus jeweils unterschiedlichen Elektronendichten. Der eindringende Teil der Strahlung wird wiederum zum Teil an der nächsten Grenzfläche reflektiert. Dadurch ergeben sich Interferenzen in der Intensität als Funktion des Einfallswinkels. Die Bedingung für Intensitätextrema ergibt sich laut [Kie31; Seg73] zu

$$
\Theta_{i}^{2}=\Theta_{t}^{2}+\frac{\left(m_{i}+\Delta m\right)^{2} \lambda^{2}}{4 d^{2}}
$$


Hier ist $\Theta_{i}$ der Einfallswinkel und $\Theta_{t}$ der Winkel, bei dem Totalreflexion an der Grenzfläche Luft - Metall auftritt. $m_{i}$ ist die ganzzahlige Ordnung des Extremums und es gilt $\Delta m=0$ für Minima und $\Delta m=1 / 2$ für Maxima, falls bei der Reflexion an der Grenzfläche Film - Substrat eine Phasensprung von $\lambda / 2$ auftritt. Wenn bei der Reflexion kein Phasensprung auftritt, gilt $\Delta m=0$ für Maxima und $\Delta m=1 / 2$ für Minima. Bei Mehrfachschichten überlagern sich die entsprechenden Oszillationen. Außerdem gibt die Abnahme der Intensität als Funktion des Einfallswinkel Aufschluss über die Rauigkeit der Grenzflächen, weil diese Rauigkeit diffuse Streuung hervorruft [PT96]. Basierend auf dem Formalismus in [Par54] existieren Simulationsprogramme, die nach Vorgabe der Materialeigenschaften die Intensitätsverläufe an die experimentellen Daten anpassen und somit Werte für die Schichtdicken und Grenzflächenrauigkeiten liefern. In dieser Arbeit wird die Methode der Kleinwinkelröntgenreflektometrie hauptsächlich eingesetzt, um die Schwingquarzwaagen zu eichen. Dies wurde zum einen über Gleichung 2.2 und zum anderen mit dem Simulationsprogramm REFSIM ${ }^{5}$ durchgeführt. Diese Methode zur Schichtdickenbestimmung ist nur für ein begrenztes Intervall von Schichtdicken anwendbar. Für sehr dünne Schichten (einige nm) wird die Oszillationsperiode sehr groß, so dass aufgrund des Intensitätsabfalls nicht mehrere Maxima bzw. Minima bestimmt werden können. Für sehr dicke Schichten $(d>150 \mathrm{~nm})$ ist dagegen die Oszillationsperiode sehr klein, so dass die Winkelauflösung des Diffraktometers nicht mehr ausreichend ist.

\subsubsection{Weitwinkelröntgendiffraktometrie}

Die Weitwinkelröntgendiffraktometrie wird in dieser Arbeit eingesetzt, um Informationen über die innere Struktur einer Probe zu gewinnen. Insbesondere soll untersucht werden, ob die Probe kristallin oder amorph ist. Gemessen wird auch hier in $\Theta / 2 \Theta$ Geometrie. Daher steht der Streuvektor wiederum in Richtung der Oberflächennormalen und man gewinnt Informationen bezüglich dieser Richtung. Um zu verhindern, dass das einkristalline Si-Substrat die Ergebnisse dominiert, wird die Probe um $\Theta=4^{\circ}$ verkippt. Sofern die metallische Schicht nicht epitaktisch ist, unterdrückt diese Verkippung nur die Reflexe des Substrats und nicht die der metallischen Schicht. Im Falle von kristallinen Strukturen erwartet man scharfe Reflexe gemäß der Bragg-Bedingung [Cul78]

$$
n \lambda=2 d_{h k l} \sin \Theta .
$$

Hier ist $n$ die Beugungsordnung des Reflexes, $\Theta$ der Einfallswinkel und $d_{h k l}$ der Netzebenenabstand entlang der durch die Millerschen Indizes gekennzeichneten Richtung in der Kristallstruktur. Die Peakbreite ist dabei nach der Scherrer-Formel umgekehrt proportional zur Korngröße der Kristallstruktur [Cul78]. Somit erwartet man für amorphe Strukturen sehr breite Peaks, die sich aus der Nahordnung nächster Nachbarn in Abwesenheit einer Fernordnung ergeben [El190]. Im amorphen Zustand trägt also neben thermischen Schwingungen vor allem die topologische Unordnung mit einem deutlich größeren Beitrag zur Verbreiterung der Intensitätsmaxima bei, während zugleich die vorhandene Nahordnung weiterhin für die Existenz der Maxima verantwortlich ist.

\footnotetext{
${ }^{5}$ Version 2.0, Bruker AXS GmbH, Karlsruhe
} 


\subsubsection{Rutherford-Rückstreuung}

Die Rutherford-Rückstreuung (RBS6) kann als weitere Methode zur Bestimmung von Schichtdicken eingesetzt werden. Des Weiteren ist mit dieser Methode eine Überprüfung der Zusammensetzung der Schicht möglich. Bezüglich der Schichtdickenbestimmung ist der Vorteil gegenüber der Kleinwinkelröntgenreflektometrie, dass dickere Schichten vermessen werden können. Die Grundlagen der Rutherford-Rückstreuung sowie die Anwendung der Methode zur Bestimmung von Schichtdicken und chemischer Zusammensetzung sind in der Literatur ausführlich beschrieben [SW92], so dass an dieser Stelle keine detaillierte Beschreibung erfolgen soll. Das zugrundeliegende Prinzip besteht darin, Projektile auf die Schicht zu schießen und aus der Energieverteilung der rückgestreuten Teilchen auf den Ort des Streuprozesses und das Element, mit dem der Streuprozess stattfindet, zu schließen. Die Vermessung der Schichten mit dieser Methode ist von H. Zutz am II. Physikalischen Institut in Göttingen durchgeführt worden. Als Projektile dienen $\mathrm{He}^{++}$Ionen mit einer Einschussenergie von $900 \mathrm{keV}$, die sich durch Anlegen einer elektrischen Spannung von $450 \mathrm{kV}$ ergibt. Die Messung wird am Beschleuniger IONAS durchgeführt [UPB $\left.{ }^{+} 85\right]$. Hier befindet sich der Detektor relativ zum einfallenden Strahl bei $165^{\circ}$ (180 ${ }^{\circ}$ entspricht Rückstreuung). Die Schichteigenschaften wie Dicke und Zusammensetzung ergeben sich durch eine Simulation mit dem Programm RUMP [Com04]. Dieses Simulationsprogramm basiert auf einem Algorithmus, in dem für eine vorgegebene Struktur das RBS-Spektrum berechnet wird und durch Iteration der Parametersatz bestimmt wird, mit dem die Abweichung von den experimentellen Daten minimiert wird [Doo86].

\subsubsection{Auswertung experimenteller Daten}

In diesem Abschnitt soll kurz beschrieben werden, wie die experimentell gewonnenen Daten sinnvoll ausgewertet und dargestellt werden können. Für die Röntgenmessungen wird lediglich die Intensität als Funktion des Einfallswinkels aufgetragen, was keiner weiteren Erläuterung bedarf. Etwas aufwendiger ist die Auswertung der mit dem Rastertunnelmikroskop gewonnenen Daten. Die Messung liefert hier jeweils eine Höheninformation für die jeweilige Substratkoordinate, d.h. man erhält eine Höhenfunktion $h(\vec{x})$ mit $\vec{x}=(x, y)$. Diese Höhenfunktion läßt sich um eine konstante skalare Zahl verschieben, so dass man $\langle h(\vec{x})\rangle_{\vec{x}}=0$ erreicht, wobei \langle\rangle$_{\vec{x}}$ Mittelung über die Substratkoordinaten bedeutet. Aus dieser Höhenfunktion lassen sich nun verschiedene Größen bzw. Funktionen berechnen:

- Die rms-Rauigkeit ( $\mathrm{rms}=$ root mean square):

$$
\sigma=\sqrt{\left\langle h^{2}(\vec{x})\right\rangle_{\vec{x}}}
$$

- Die Höhen-Höhen-Korrelationsfunktion (im Realraum):

$$
C(\vec{r})=\langle h(\vec{x}) \cdot h(\vec{x}+\vec{r})\rangle_{\vec{x}} .
$$

\footnotetext{
${ }^{6}$ Englisch: Rutherford backscattering
} 
Der Rechenaufwand ist allerdings kleiner, wenn man die Beziehung

$$
C(\vec{r})=F T^{-1}\left[|F T[h(\vec{r})]|^{2}\right]
$$

benutzt [Rei97; May97]. Dabei bedeutet FT die Fouriertransformation und $F T^{-1}$ ihr Inverses. Es gilt insbesondere $C(0)=\sigma^{2}$. Bei isotropen Bildern ist es weiterhin sinnvoll, die Höhen-Höhen-Korrelationfunktion azimuthal zu mitteln:

$$
C(r)=\langle C(\vec{r})\rangle_{|\vec{r}|=r} .
$$

Diese Funktion beschreibt die Stärke periodisch wiederkehrender Anordnungen in der Abbildung, die wiederum sowohl durch die Oberfläche selbst als auch durch die Form der Spitze bedingt sein können, da die Abbildung immer eine Faltung aus Spitze und Oberfläche ist. Nimmt man an, dass die Spitze keine starken Artefakte aufweist, so kann man anhand der Höhen-Höhen-Korrelation ein quantitatives Maß für einen Hügeldurchmesser und auch für den durchschnittlichen Abstand zweier Hügel bestimmen. Der typische Hügelabstand entspricht dem Achsenabschnitt $r=R_{c}$, bei dem die Korrelationsfunktion ihr erstes Nebenmaximum hat. Bei einer angenommenen Gaußverteilung erhält man ein Maß für den typischen Hügelradius durch den Achsenabschnitt $r=R_{e}$, bei dem ein Gaußfit an die Korrelationsfunktion im Bereich des Hauptmaximums auf 1/e abgefallen ist [ $\left.\mathrm{RVR}^{+} 82\right]$.

- Die spektrale Leistungsdichte:

$$
C(\vec{q})=|F T[h(\vec{x})]|^{2}
$$

Auch für diese Korrelationsfunktion im Fourierraum ist eine azimuthale Mittelung bei isotropen Strukturen sinnvoll:

$$
C(q)=\langle C(\vec{q})\rangle_{|\vec{q}|=q}
$$

- Die Höhen-Differenz-Korrelationsfunktion:

$$
H(\vec{r})=\left\langle|h(\vec{x})-h(\vec{x}+\vec{r})|^{2}\right\rangle_{\vec{x}}
$$

Dieses Funktion läßt sich alternativ aus zuvor definierten Größen bestimmen über die Beziehung [Rei97]

$$
H(\vec{r})=2\left(\sigma^{2}-C(\vec{r})\right) .
$$

Wie zuvor ergibt sich durch azimuthale Mittelung dieser Funktion:

$$
H(r)=\langle H(\vec{r})\rangle_{|\vec{r}|=r}
$$

Für die konkrete Art der Berechnung dieser Größen, insbesondere derjenigen, die von der Fouriertransformation Gebrauch machen, sind Details in [Str04] beschrieben. Insbesondere sind dort die Unterschiede der eigentlichen kontinuierlichen Fouriertransformation zu der für sämtliche diskrete Daten aus einem gerasterten Bild in der Computersoftware verwendeten FFT (diskrete 'Fast Fourier Transform') erläutert. Die STM-Daten 
wurden mit der auf der Programmiersprache IDL $^{7}$ basierenden Software XOM, die von C. Streng geschrieben wurde [Str04], ausgewertet. Für die Berechnung der radial gemittelten Korrelationsfunktionen der Daten aus Simulationen im Kontinuumsmodell wird die von S. G. Mayr und B. M. Reinker entwickelte Software [Rei97; May97], die ebenfalls in IDL geschrieben ist, verwendet.

${ }^{7}$ Interactive Data Language 


\section{Molekulardynamik-Simulationen}

\subsection{Ziel der Molekulardynamik-Simulationen}

Das Ziel der im Rahmen dieser Arbeit durchgeführten Molekulardynamik-Simulationen (MD-Simulationen) ist die Untersuchung der Dynamik im Bereich der Oberfläche von Gläsern bei Anregung durch Einschuss energetischer Atome. Zum Vergleich werden auch Prozesse bei thermischer Anregung analysiert, um Unterschiede und Gemeinsamkeiten festzustellen. Eine Verbindung zwischen beiden Arten der Anregung ist in der Literatur außerdem durch die Vorstellung von „thermal spikes“, also der lokalen Temperaturerhöhung, als Folge von Ionenbestrahlung diskutiert [SK56; AR98]. Um möglichst allgemeine Aussagen treffen zu können, werden zwei verschiedene Materialklassen untersucht, nämlich das eher fragile, metallische Glas CuTi und das starke, kovalent gebundene Netzwerkglas Si.

Durch die atomistische Simulation soll ein Einblick in die mikroskopischen Prozesse gewonnen werden. Damit bildet dieser Teil der Arbeit das Komplement zu den experimentellen Untersuchungen und der Modellierung durch stochastische Ratengleichungen, in denen auf mesoskopischer Skala die Folgen der mikroskopischen Prozesse beobachtet werden. Um die Verbindung zwischen den verschiedenen Skalen herstellen zu können, ist es nötig, die Daten aus der Simulation im Sinne einer multiskaligen Modellierung entsprechend auszuwerten. Die dafür relevanten Größen werden im weiteren Verlauf der Arbeit vor der Darstellung der zugehörigen Ergebnisse definiert. In diesem Kapitel werden nun zunächst die grundsätzlichen Methoden und Eigenschaften der MD-Simulation erläutert.

\subsection{Grundlagen}

Die Molekulardynamik beschreibt die Bewegung aller Atome in einem abgeschlossenen System. Zur Vereinfachung kann man das zu beschreibende System in der BornOppenheimer Näherung betrachten, d.h. es wird berücksichtigt, dass die Atomkerne deutlich träger sind als die Elektronen, was zur Folge hat, dass das elektronische System den Atomkernen quasi instantan folgt. Diese Näherung wird sowohl in klassischen als auch in quantenmechanischen MD-Simulationen verwendet. In der klassischen MDSimulation wird auf eine explizite Modellierung der elektronischen Vorgänge verzichtet und der Einfluss der Elektronen wird lediglich als gemittelter Effekt, z.B. in Form einer modellierten Elektronendichte, im Wechselwirkungspotential $V$ der Kerne berücksichtigt. Diese Methode liefert ausreichende Informationen, wenn die Bewegung der Atome untersucht werden soll und die vollständigen elektronischen Eigenschaften nicht von weiterem 
Interesse sind. Die Alternative hierzu ist, das gesamte System inklusive der Elektronen durch quantenmechanische ab initio Berechnungen zu beschreiben (siehe z.B. [Sch06]). Da diese Methode sehr rechenaufwendig ist, können mit ihr nur kleine Systeme bzw. sehr kurze Zeiten erschlossen werden. In dieser Arbeit sind aber Systemgrößen und Simulationszeiten nötig, die nur mit klasssischer MD-Simulation erreichbar sind. Außerdem beziehen sich die Untersuchungen auf die Bewegungen der Atome, so dass die klassische MD-Simulation ausreichend ist und es im Folgenden nur noch um diese Methode gehen wird.

Um das Zusammenspiel vieler Atome nun zu untersuchen, muss man prinzipiell ein Vielkörperproblem lösen, d.h. die Newtonschen Bewegungsgleichungen müssen für $\mathrm{N}$ Teilchen, die miteinander wechselwirken, gelöst werden. Das entspricht der Lösung eines Systems von N gekoppelten Differentialgleichungen zweiter Ordnung:

$$
m_{i} \ddot{\overrightarrow{r_{i}}}=-\vec{\nabla}_{\vec{r}_{i}} V\left(\vec{r}_{1}, \ldots, \vec{r}_{N}\right) \quad i=1, \ldots, N
$$

Dies ist auch bei Kenntnis aller Wechselwirkungen der Atome im Allgemeinen analytisch nicht möglich. Es bietet sich an, ein solches Problem numerisch zu lösen, wobei solche Methoden immer approximativ sind, da für die Lösung Ort und Zeit diskretisiert werden. Die Lösung der Gleichungen erfolgt über die Methode der finiten Differenzen, für die verschiedene Integrationsalgorithmen in der Literatur diskutiert werden (siehe z.B. [Rap95; AT87]). Damit lassen sich dann also die mikroskopischen Prozesse der Atome in numerischer Näherung berechnen. Von dieser mikroskopischen Ebene kann man dann mit Hilfe der Gesetze der statistischen Mechanik Erkenntnisse über makroskopische Größen erlangen. Aufgrund der approximativen Lösung der Bewegungsgleichungen und der sensitiven Abhängigkeit von den Anfangsbedingungen können die simulierten Trajektorien einzelner Atome von den wahren Trajektorien abweichen. Für die makroskopischen Ergebnisse und andere statistisch gemittelte Eigenschaften ergibt sich daraus aber kein großer Fehler, solange durch das Gesamtsystem ein ausreichender Anteil des Phasenraums hinreichend genau durchfahren wird [Sch06].

Für das Wechselwirkungspotential gibt es verschiedene Ansätze, die natürlich auch von der zu simulierenden Materialklasse abhängig sind. In der klassischen Molekulardynamik kommen empirische Potentiale zum Einsatz. Dabei sind neben Potentialen, die nur Paar-Wechselwirkungen einbeziehen auch Potentiale möglich, die Vielteilcheneffekte berücksichtigen. Letztere können im Gegensatz zu reinen Paar-Potentialen auch z.B. von der Dichte des Systems abhängen. Da die Berechnung der Kraft in der MD-Simulation ein sehr aufwendiger Schritt bezüglich der Rechenzeit ist, berücksichtigt man in der Regel nur Wechselwirkungen von Atomen, deren Abstand unterhalb eines vom System abhängigen Wertes $r_{c}$ liegt. Die genaue Form eines Potentials bzw. dessen Parameter werden bei der Entwicklung so angepasst, dass experimentell zugängliche Größen möglichst gut wiedergegeben werden.

Soweit wie bisher beschrieben, simuliert man ein System einer konstanten Anzahl von Atomen $\mathrm{N}$ in einem konstanten Volumen V. Aufgrund der Newtonschen Bewegungsgleichungen bleibt in diesem System außerdem die Gesamtenergie des Systems E erhalten. Falls das System ergodisch ist, hat man damit ein mikrokanonisches (NVE) Ensemble. 
Um Vergleiche mit Experimenten zu ermöglichen, ist es aber oft sinnvoll, andere thermodynamische Ensembles zu betrachten. Insbesondere ist es oft sinnvoll, die Temperatur statt der Energie und den Druck statt des Volumens vorzugeben ((NPT) Ensemble). Dies kann durch Kopplung des Systems an ein Wärme- bzw. Druckreservoir erreicht werden (siehe Kap. 3.3.1).

Die prinzipielle Herangehensweise bei MD-Simulationen ist beispielsweise in [Rap95; AT87; Win06] ausführlich beschrieben.

\subsection{Eigenschaften des verwendeten Simulationsprogramms}

In dieser Arbeit wird ein von S. G. Mayr weiterentwickelter Programmcode benutzt, der teilweise auf früheren Versionen von PARCAS und ALCMD basiert 1 . Dabei handelt es sich um einen parallelisierten Simulationscode, so dass der Einsatz von Parallelrechnern möglich ist. Es sind u.a. verschiedene Typen von Potentialen implementiert, eine Temperatur- und Druckkontrolle, sowie verschiedene Besonderheiten, die nötig sind, um Einschüsse von Atomen simulieren zu können (s.u.).

Die Integration der Bewegungsgleichungen erfolgt über die Methode der finiten Differenzen nach einem „Gear Prädiktor-Korrektor" Algorithmus, wobei Ableitungen bis zur 5. Ordnung berücksichtigt werden [AT87]. Die Zeitschrittweite $\Delta t$ der numerischen Integration muss stets klein genug sein, um eine ausreichende Genauigkeit zu gewährleisten. Andererseits vergrößern unnötig kleine Zeitschrittweiten den Rechenaufwand. Die Größe einer sinnvollen Zeitschrittweite hängt einerseits vom Wechselwirkungspotential und zum anderen insbesondere von den atomaren Geschwindigkeiten im System ab. Da gerade bei der Simulation von Ionenbeschuss teilweise sehr hohe atomare Geschwindigkeiten auftreten, ist während eines solchen Vorgangs eine sehr kleine Zeitschrittweite nötig. Um andererseits effizient zu arbeiten, sollte die Zeitschrittweite nach dem Einschuss wieder vergrößert werden. Daher wird im Simulationscode eine variable Zeitschrittweite, die aus einer vorgegebenen maximalen Zeitschrittweite, den atomaren Geschwindigkeiten und den Kräften bestimmt wird, verwendet [Nor95]. Damit ist außer der nach oben begrenzten Zeitschrittweite zusätzlich gewährleistet, dass kein Atom während eines Zeitschritts eine Verschiebung im Ort bzw. eine Änderung seiner potentiellen Energie durchführt, die größer als ein vorgegebener Wert ist. Diese Methode verbindet hinreichende Genauigkeit mit größtmöglicher Effizienz.

\subsubsection{Druck- und Temperaturkontrolle}

In den durchgeführten Simulationen ist es an vielen Stellen sinnvoll, zum einen den Druck statt eines Volumens und zum anderen die Temperatur statt der Energie vorzugeben. Dabei entspricht im Folgenden die Temperatur der kinetischen Temperatur, die

\footnotetext{
${ }^{1}$ PARCAS ist eine Weiterentwicklung des Simulationscodes ALCMD von D. Turner und J. Morris
} durch K. Nordlund. 
sich aus der mittleren kinetischen Energie durch Verwendung des Gleichverteilugssatzes ergibt [AT87]. Der Druck bzw. die mechanischen Spannungen lassen sich bei Kenntnis der kinetischen Energien über die Viriale aus der Simulation berechnen [AT87], wobei hier jeweils die Anteile der Größen für die einzelnen Raumrichtungen betrachtetet werden müssen. Die Möglichkeit, den Druck bzw. die Temperatur vorzugeben, wird über einen Reskalierungsmechanismus nach Berendsen et al. realisiert [BPG $\left.{ }^{+} 84\right]$. So wird eine vorgegebene Temperatur $T_{0}$ angenähert durch die Reskalierung der Geschwindigkeiten $\overrightarrow{v_{i}}$ der durch $i$ indizierten Atome pro Zeitschritt $\Delta t$ :

$$
\overrightarrow{v_{i}} \mapsto \sqrt{1+\frac{\Delta t}{\tau_{T}}\left(\frac{T_{0}}{T}-1\right)} \vec{v}_{i} .
$$

Dabei ist $T$ die momentane Temperatur und $\tau_{T}$ die Zeitkonstante der Reskalierung. Die Druckkontrolle funktioniert insofern ähnlich, als hier die Ortsvektoren reskaliert werden:

$$
x_{i} \mapsto\left(1-\frac{\beta \Delta t\left(p_{x x}^{0}-p_{x x}\right)}{3 \tau_{p}}\right) x_{i} .
$$

Hier sind $p_{x x}$ und $p_{x x}^{0}$ der momentane und der gewünschte Druck bzw. die mechanische Spannung in x-Richtung, $x_{i}$ die x-Komponente des Ortsvektors des durch i indizierten Atoms, $\beta$ ist die isotherme Kompressibilität und $\tau_{p}$ ist die Zeitkonstante für die Reskalierung. Für alle drei Raumrichtungen wird die mechanische Spannung ausgewertet und ein gewünschter Wert $p_{x x}^{0}, p_{y y}^{0}, p_{z z}^{0}$ wird vorgegeben, so dass die Regulierung der Spannungen gemäß Gl. (3.3) bzw. der analogen Gleichungen für die anderen Richtungen erfolgt. Damit lassen sich sowohl ein isotroper Druck als auch je nach Raumrichtung verschiedene mechanische Spannungen realisieren.

Diese Methode erzeugt zwar kein thermodynamisches Ensemble, aber sie ist nützlich, um einen neuen Zustand einzustellen und diesen dann auch schnell equilibrieren zu lassen, da die Fluktuationen sehr klein sind [AT87]. Insbesondere werden die Mittelwerte, aber nicht die Fluktuationen, der thermodynamischen Zustandsgrößen korrekt wiedergegeben. Da diese Fluktuationen nicht Untersuchungsgegenstand dieser Arbeit sind, ist die Methode hier ohne Beeinträchtigung der Ergebnisse anwendbar. Die BerendsenTemperaturkontrolle zeigt insbesondere auch bei der Simulation von energetischem Teilchenbeschuss zufriedenstellende Ergebnisse [HS04].

Des Weiteren erlaubt der Simulationscode verschiedene Modi der Temperaturkontrolle. So kann die Temperatur der Gesamtprobe bei konstanter Rate $\dot{T}$ einer vorgegebenen Temperatur angenähert werden. Dieser Modus wird beispielsweise zur Herstellung amorpher Proben genutzt, um die Probe aus der flüssigen Phase abzuschrecken. Natürlich kann die Temperatur auch konstant beim Startwert gehalten werden, was zur Equilibrierung und Relaxation eines Systems sinnvoll ist. Bei Bestrahlungen kann ein Modus genutzt werden, bei dem die Temperaturkontrolle nur auf Atome einer dünnen Schicht an den Boxrändern angewendet wird, denn das entspricht der Abführung der thermischen Energie nach außen. Außerdem werden gesputterte Atome, die außerhalb der Probe sind und eine hohe Geschwindigkeit haben, nicht für die Temperaturkontrolle berücksichtigt. 


\subsubsection{Wechselwirkungspotentiale}

In dieser Arbeit werden in der MD-Simulation zwei verschiedene Materialien, die auch unterschiedlichen Materialklassen zuzuordnen sind, untersucht. Daher sind die Gemeinsamkeiten beider Systeme für eine große Klasse von Gläsern relevant. Das Konzept besteht also darin, zwei zuverlässige Modellsysteme zu untersuchen, um für viele Gläser gültige Eigenschaften identifizieren zu können. Die Wahl der Materialien ergibt sich zunächst daraus, dass für das experimentell verwendete System $\mathrm{Zr}_{65} \mathrm{Al}_{7.5} \mathrm{Cu}_{27.5}$, dessen Wahl für die Experimente in Kap. 2.2.1 begründet wird, bisher kein Potential modelliert ist. Daher soll zum einen das eher fragile metallische Glas CuTi und zum anderen das starke, kovalent gebundene Glas Si betrachtet werden, weil das experimentell verwendete System bezüglich der Fragilität zwischen diesen beiden Systemen liegt. Es hat sich nämlich gezeigt, dass durch Zugabe von $\mathrm{Al}$ zu einem binären metallischen Glas ein stärkeres Glas mit einem größeren Anteil kovalenter Bindungen erzeugt wird [BBJ98; GT01] und Si sollte wiederum stärker sein als beide metallischen Gläser [El190]. Somit sollten Gemeinsamkeiten der Systeme CuTi und Si auch für das experimentell betrachtete System relevant sein. Des Weiteren zeigt das CuTi System folgende Ähnlichkeiten zum ZrAlCu System: In beiden Legierungen ist $\mathrm{Cu}$ als ein Hauptbestandteil enthalten und der zweite Hauptbestandteil ist chemisch ähnlich, weil $\mathrm{Zr}$ und Ti im Periodensystem in der gleichen Gruppe stehen. Außerdem zeigen die metallischen Gläser $\mathrm{CuTi}$ und $\mathrm{Zr}_{65} \mathrm{Al}_{7.5} \mathrm{Cu}_{27.5}$ große Übereinstimmung in ihrem kinetischen Verhalten, das die Oberflächenstruktur während des Wachstums dünner Schichten beeinflusst $\left[\mathrm{RML}^{+} 00 ;\right.$ Hül98].

Für beide Materialien, also $\mathrm{CuTi}$ und $\mathrm{Si}$, werden Mehrkörperpotentiale verwendet, die über Paarkräfte hinausgehende Wechselwirkungen berücksichtigen. Da Potentiale in der Regel für atomare Positionen in der Nähe des Gleichgewichts entwickelt werden, ist noch eine Modifikation solcher Potentiale nötig, wenn energetische Bestrahlung untersucht werden soll. Durch Stöße und hohe Energie, können sich Atome nämlich sehr nahe kommen und ihre Gleichgewichtspositionen deutlich verlassen, so dass die ursprünglichen Potentiale aus der Literatur im Bereich kurzer Abstände realistischer modelliert werden müssen. Diese Änderungen sind aber im verwendeten Simulationscode bereits enthalten (s.u.).

\subsubsection{Das CuTi Potential}

Für die metallische Legierung CuTi wird ein Potential von Sabochick und Lam verwendet [SL91]. Es basiert auf der sogenannten 'embedded atom method' (EAM). Bei dieser Methode, die auf Daw und Baskes zurückgeht [DB83; DB84], nimmt man zum einen eine abstoßende Paarwechselwirkung zwischen den positiv geladenen Atomkernen an. Hinzu kommt noch eine anziehende Kraft, die durch die im Hintergrund verteilten Elektronen auf die Kerne ausgeübt wird. Die Energie im Gesamtsystem ergibt sich in einem solchen Modell für eine Legierung entsprechend zu [SL91]:

$$
E_{p o t}=\sum_{i} F_{k}\left[\rho\left(\mathbf{r}_{i}\right)\right]+\sum_{i<j} \Phi_{k m}\left(r_{i j}\right) .
$$


Dabei ist dann $F$ die Einbettungsfunktion für die Atome in den Hintergrund aus Elektronen, $\mathbf{r}_{i}$ die Position von Atom $i, r_{i j}$ der Abstand zwischen Atom $i$ und Atom $j, \Phi$ das abstoßende Paarpotential zwischen den Atomkernen und $\rho$ die durch alle anderen Atome induzierte Elektronendichte am Ort des Atoms $i$. Durch $k$ bzw. $m$ ist die Atomsorte bei einer Legierung gekennzeichnet. Dabei ergibt sich die Elektronendichte am Ort des Atoms $i$ als Superposition der Elektronendichten aller anderen Atome an diesem Ort gemäß $\rho\left(\mathbf{r}_{i}\right)=\sum_{j \neq i} f_{m}\left(r_{i, j}\right)$, wobei $f_{m}$ die Elektronenverteilung eines Atoms der Sorte $m$ beschreibt. Wegen dieser von allen anderen Atomen induzierten Elektronendichte ist das Modell ein Vielteilchen-Potential. Die genaue Form des Potentials wird dann in [SL91] so angepasst, dass Größen wie das atomare Volumen, elastische Konstanten, Leerstellenbildung- und Leerstellenwanderungsenergien möglichst gut mit experimentellen Daten übereinstimmen. Das für CuTi angepasste Potential aus [SL91] wird in dieser Arbeit auch für leicht abweichende Zusammensetzungen, nämlich $\mathrm{Cu}_{40} \mathrm{Ti}_{60}$ und $\mathrm{Cu}_{60} \mathrm{Ti}_{40}$ verwendet.

Ein Vorteil des EAM-Potentials gegenüber reinen Paar-Potentialen ist, dass lokale Änderungen der Konfiguration wie z. B. Dichteänderungen aufgrund der Berücksichtigung von Vielteilcheneffekten besser berücksichtigt werden, weshalb es sich auch für die Beschreibung von Oberflächen eignet. Für Oberflächen wurde nämlich das Zusammenziehen der obersten Lage beobachtet, wie es auch experimentell bekannt ist [FBD86]. Außerdem ermöglicht das Modell in einer Legierung die Ausbildung chemischer Ordnung, da die Einbettungsfunktionen und die von einem Atom ausgehende Elektronendichte von der Atomsorte abhängig sind. Des Weiteren ist auch bekannt, dass EAM-Potentiale den Zustand der Flüssigkeit für Übergangsmetalle gut beschreiben können [Foi85; BSS96]. Als Potential, das sowohl die Flüssigkeit als auch Oberflächen gut beschreibt, erfüllt es die wesentlichen Anforderungen für die Simulationen in dieser Arbeit. Es werden nämlich dünne Schichten mit Oberflächen, amorphe Strukturen, die sich durch Abschrecken aus der Schmelze ergeben, sowie in Folge der Bestrahlung auch lokal flüssige Bereiche untersucht. Allerdings sind in diesem Modell keine gerichteten Bindungsanteile enthalten, was bei einem Vergleich zu experimentell untersuchten metallischen Gläsern bedacht werden muss.

Die für die Simulation energetischer Ionenbestrahlung nötige Änderung des Potentials im Bereich kurzer Abstände ist von S. G. Mayr gemäß dem universellen Ziegler-BiersackLittmark Potential [ZBL85] angepasst, wie in [MAAA03] beschrieben. Der Abschneideradius für große Abstände ist weiterhin wie in [SL91] gewählt.

Mit dem gewählten Integrationsalgorithmus hat sich als hinreichend kleine Obergrenze der variablen Zeitschrittweite $\Delta t=3.52$ fs erwiesen [May03]. Weiterhin ist gewährleistet, dass die Zeitschrittweite stets so klein, dass keine atomaren Verschiebungen mit $|\Delta \vec{x}|>0.1 \AA$ in einem Zeitschritt auftreten.

\subsubsection{Das Si Potential}

Für die Simulation von Silizium wird ein Potential verwendet, das auf dem von Tersoff entwickelten Modell [Ter86] basiert. In diesem Modell, das von Tersoff in [Ter88b] erweitert und ausführlicher motiviert wird, wird die Wechselwirkungsenergie zunächst formal 
in der Form eines Paarpotentials geschrieben:

$$
E_{p o t}=\frac{1}{2} \sum_{i, j \neq i} V_{i j}, \quad V_{i j}=f_{c}\left(r_{i j}\right)\left[A \exp \left(-\lambda_{1} r_{i j}\right)-B_{i j} \exp \left(-\lambda_{2} r_{i j}\right)\right]
$$

Dabei ist $r_{i j}$ wieder der Abstand zwischen Atom $i$ und Atom $j, A$ und $\lambda_{1,2}$ sind positive Konstanten und $f_{c}$ ist eine Abschneidefunktion, um das Potential für große Abstände mit glattem Übergang auf null zu setzen. Während der erste Term in $V_{i j}$ abstoßend ist, beschreibt der zweite Term die Anziehung und muss damit den kovalenten Bindungscharakter wiedergeben. Das bedeutet aber, dass $B_{i j}$ von der lokalen Umgebung abhängig sein muss, denn kovalente Bindungsanteile hängen von der lokalen Umgebung ab und ermöglichen so auch weniger dicht gepackte stabile Strukturen. Somit gibt es einen Zusammenhang zwischen der Koordinationszahl und dem Wert von $B_{i j}$. Wegen der Abhängigkeit von $B_{i j}$ von der lokalen Umgebung stellt das Modell ein Vielkörperpotential dar. Die genaue Parametrisierung des in der Simulation verwendeten Potentials wurde ebenfalls von Tersoff entwickelt und ergibt sich aus einer Anpassung eines verfeinerten Modells an experimentelle Ergebnisse und aus quantenmechanischen ab initio Berechnungen stammende Daten [Ter88a]. Von den verschiedenen dort angegebenen Parametrisierungen wird in dieser Arbeit das $\mathrm{Si}(\mathrm{C})$ Potential $\left(\right.$ mit $\left.\lambda_{3} \neq 0\right)$ verwendet. In dieser Parametrisierung werden zum einen die elastischen Eigenschaften und zum anderen die Eigenschaften von flüssigem Silizium besser wiedergegeben. Die Struktur in der flüssigen Phase ist für die hier durchgeführten Simulationen wichtig, da der amorphe Zustand sich durch rasches Abschrecken der Flüssigkeit ergibt und außerdem lokal flüssige Bereiche durch Bestrahlung entstehen können (s.u.).

Der Vorteil des Modells von Tersoff ist, dass es auch nichttetraedrische Bindungsformen in der festen Gleichgewichtsphase prinzipiell zulässt im Gegensatz zu anderen Potentialen, die als Erweiterung zu Paarkräften eine Dreikörper-Bindungswinkel-Wechselwirkung einführen, die dann aber den gewünschten Bindungswinkel in der festen Gleichgewichtsphase im Wesentlichen festlegt [SW85]. Insofern hat das Tersoff-Modell insbesondere an Oberflächen, wo durch Rekonstruktion andere Bindungsformen erwartet werden entscheidende Vorteile.

Wie im vorigen Abschnitt für CuTi muss auch im Falle von Si das verwendete empirische Potential für kurze Reichweiten modifiziert werden. Im ursprünglichen Modell wird nämlich wieder davon ausgegangen, dass sich die Atome in der Nähe der Gleichgewichtspositionen befinden und das gilt beim Einschuss energiereicher Atome nicht immer. Daher wird das Potential für kurze Reichweiten durch ein aus ab initio Rechnungen bekanntes abstoßendes Paarpotential ersetzt [NA97; NGA+98]. Der Abschneideradius für große Abstände ist weiterhin wie in [Ter88a] gewählt.

Hier hat sich mit dem gewählten Integrationsalgorithmus als hinreichend kleine Obergrenze der variablen Zeitschrittweite $\Delta t=2.70 \mathrm{fs}$ erwiesen [May05b]. Wie beim CuTi ist auch hier außerdem gewährleistet, dass während eines Zeitschritts keine Verschiebungen mit $|\Delta \vec{x}|>0.1 \AA$ vorkommen. 


\subsubsection{Zusätzliche Wechselwirkungen beim Einschuss von Atomen}

So wie bisher beschrieben würden eingeschossene Atome in der verwendeten klassischen MD-Simulation nur mit den Atomkernen wechselwirken, da nur deren Dynamik berechnet wird. Die Elektronen gehen nur implizit als Mittelung zur Berechnung der Dynamik der Kerne ein. In Abhängigkeit von der Einschussenergie sind in der Realität aber neben den Atomkernen auch die Elektronen explizit für das Abbremsen des eingeschossenen Atoms verantwortlich [AR98]. Das eingeschossene Atom kann nämlich zum einen durch elastische Stöße mit den anderen Kernen und zum anderen durch Anregung des elektronischen Systems Energie abgeben. Im verwendeten Simulationsprogramm ist das Abbremsen der Atome durch das elektronische System als eine zusätzliche Reibungskraft in der Bewegungsgleichung implementiert. Der Reibungsterm wird aber nur für solche Atome hinzugefügt, deren kinetische Energie $10 \mathrm{eV}$ überschreitet. Dadurch wird verhindert, dass thermische Schwingungen ebenfalls gedämpft werden. Der Wert der Reibungskraft basiert auf für verschiedene Geschwindigkeiten tabellierten Werten aus SRIM [ZB03]. Zwischen den tabellierten Geschwindigkeiten wird die Reibungskraft linear interpoliert, wie in [May05a] beschrieben.

\subsection{Probenherstellung}

An dieser Stelle soll erläutert werden, wie die Proben hergestellt werden. Die prinzipielle Art der Herstellung ist für alle Proben gleich. Unterschiede gibt es in einigen Details, die daher jeweils im Zusammenhang mit den zugehörigen Ergebnisse dargestellt sind. Ein schematischer Überblick ist in Abb. 3.1 gegeben.

Um eine definierte Anfangskonfiguration bezüglich der Ortskoordinaten zu haben, werden die Atome zu Beginn entsprechend ihrer Gleichgewichtsposition in der Kristallstruktur angeordnet. Im Falle von $\mathrm{Cu}_{50} \mathrm{Ti}_{50}$ ist das die B11 Struktur für die $\gamma$-Phase [SL91]. Für die Simulation von $\mathrm{Cu}_{40} \mathrm{Ti}_{60}$ sowie $\mathrm{Cu}_{60} \mathrm{Ti}_{40}$ ist die $\mathrm{Cu}_{50} \mathrm{Ti}_{50}$-Konfiguration der Ausgangspunkt. Zufällig gewählte Atome der Sorte, deren Anteil verringert werden soll, werden durch die andere Atomsorte ersetzt. Beim Si wird eine aus vorangegangenen Simulationen bestehende ungeordnete Probe als Startkonfiguration verwendet. Als Starttemperatur wird für alle Proben $T=10 \mathrm{~K}$ gewählt und die Beträge der atomaren Geschwindigkeiten sind bei neuer Initialisierung um den dieser thermischen Energie entsprechenden Mittelwert herum gaußverteilt, während die zugehörigen Richtungen gleichverteilt sind, so dass die Startwerte für die Impulse gegeben sind. In alle Richtungen werden periodische Randbedingungen angelegt und der Druck wird istrop zu $p=0$ mbar geregelt. Unter diesen Randbedingungen wird die Probe dann sehr schnell auf ein Vielfaches der Schmelztemperatur geheizt. Im Fall aller Legierungen aus $\mathrm{Cu}$ und $\mathrm{Ti}$ wird $T_{\max }=6000 \mathrm{~K}$ und für Si wird $T_{\max }=7000 \mathrm{~K}$ gewählt. In dieser flüssigen Phase lässt man das System nun für einige Zeit equilibrieren. Es sollte zumindest gewährleistet sein, dass sich eine Flüssigkeit mit gleichmäßiger Temperatur gebildet hat. Wenn sich so ein Gleichgewicht eingestellt hat, hat die zuvor verwendete Heizrate keinen Einfluss mehr auf die weitere Entwicklung. Danach erfolgt das Abkühlen mit einer konstanten Tempe- 


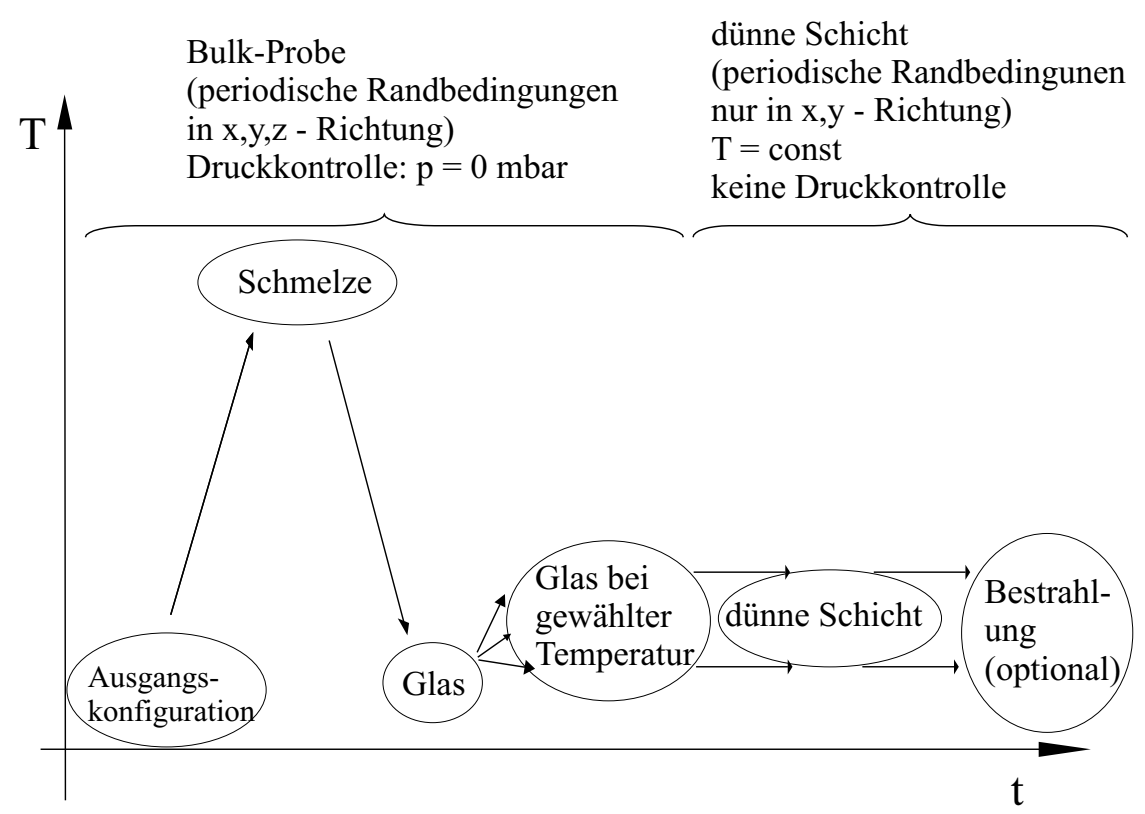

Abbildung 3.1: Schematische Darstellung der Probenherstellung in der MD-Simulation im Temperatur-Zeit Diagramm.

raturrate herunter bis $T=0 \mathrm{~K}$ oder $T=10 \mathrm{~K}(\dot{T}=20 \mathrm{~K} / \mathrm{ps}$ für die CuTi Legierungen und $\dot{T}=50 \mathrm{~K} / \mathrm{ps}$ für $\mathrm{Si}$ ) mit einer sich daran anschließenden Zeit, die das System unter diesen konstanten Bedingungen bleibt. Mit dieser Methode hat man durch „Abschrecken aus der Schmelze“ ein Glas erzeugt, wie man es ähnlich, allerdings mit anderen Raten, auch im realen Experiment tun könnte [Zar91]. Gegebenenfalls wird danach das System wieder geheizt, um die Endtemperatur zu erreichen, bei der die jeweilige Untersuchung stattfinden soll. Die Heizrate ist dann immer die gleiche wie zuvor die Abkühlrate. Auch bei der Endtemperatur bleibt die Probe wieder einige Zeit unter konstanten Bedingungen, um Relaxation zu ermöglichen. Im nächsten Schritt wird die Druckkontrolle für alle Richtungen ausgeschaltet und die periodischen Randbedingungen werden in einer Richtung (hier immer die z-Richtung) aufgehoben. Dadurch ergeben sich zwei Oberflächen in der z-Richtung, während die Probe in $\mathrm{x}$ - und y-Richtung periodisch fortgesetzt ist (siehe Abb. 3.2). Diese Geometrie entspricht der einer dünnen Schicht, wobei diese in der Simulation sozusagen frei schwebt und insbesondere ohne Substrat existiert. Dabei sind die Oberflächen glatt, wenn man von atomaren Rauigkeiten absieht. Die so hergestellten Schichten werden wiederum für eine gewisse Zeit relaxiert, bevor die eigentliche Untersuchung beginnt. Für die Untersuchungen zu thermisch aktivierten Prozessen findet kein weiterer Eingriff in das System statt.

Für die Untersuchungen von energetischen atomaren Einschüssen wird nun auf die, wie im vorigen Absatz beschrieben, hergestellte Schicht ein Atom geschossen. Dafür wird ein Atom in einem Abstand von typischerweise $5 \AA$ mit gewünschter oder zufällig gewählter Koordinate bezüglich der Substratebene und einer auf die Oberfläche gerichteten Geschwindigkeit über die Oberfläche gesetzt. Dieser Einschuss kann entweder senkrecht oder schräg zur Oberfläche sein, wobei sich bei schrägem Einschuss das Atom 


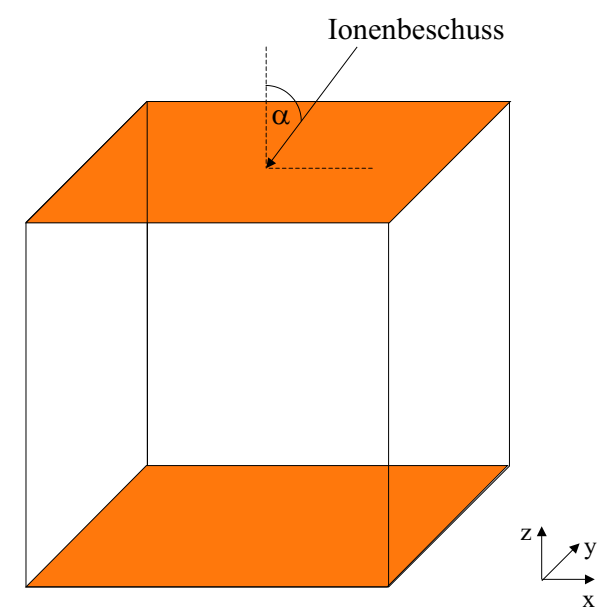

Abbildung 3.2: Zelle für die Simulation dünner Schichten; an weißen Fläche herrschen periodische und an orangen Flächen offene Randbedingungen.

entlang der x-Achse bewegt, so dass die y-Komponente der Geschwindigkeit stets null ist und ein Winkel $\alpha$ zur Oberflächennormalen besteht (siehe Abb. 3.2). Wegen der Isotropie der amorphen Oberfläche stellt diese Vorgehensweise keine Einschränkung dar. Für den Beschuss wird die Temperaturkontrolle in den Modus umgestellt, bei dem nur die Geschwindigkeiten der Atome, die sich in einer dünnen Schicht an den Rändern der periodisch fortgesetzten Richtungen befinden, der gewünschten Temperatur entsprechend reskaliert werden (siehe Kap. 3.3.1). Die Dicke dieser Schicht beträgt sowohl für CuTi als auch für Si $5.43 \AA$ A. Alternativ können die untersten Monolagen der von oben beschossenen Probe festgehalten und einige weitere Monolagen durch die Temperaturkontrolle reskaliert werden.

Um Randeffekte auszuschließen, muss immer bedacht werden, dass die Größe der Simulationszelle für Einschüsse einer gegebenen Energie groß genug ist. Falls nicht anders erwähnt besteht die CuTi Zelle aus 35152 Atomen und die Si Zelle aus 32768 Atomen, was einer Kantenlänge der kubischen Simulationszelle von ca. $80 \AA$ bzw. $88 \AA$ entspricht. Diese Zellen sind groß genug für Einschüsse bis $1 \mathrm{keV}$ [May05a]. Es wurden aber auch Tests mit größeren Zellen ( $\mathrm{N}=262069$ und $\mathrm{N}=524288)$ durchgeführt. 


\section{Kontinuumsmodellierung}

In diesem Kapitel soll in die Modellierung der Entwicklung von Oberflächenstrukturen durch einen kontinuumstheoretischen Ansatz eingeführt werden. Kontinuumsmodelle ermöglichen die Beschreibung von Oberflächenstrukturen auf mesoskopischer Skala und sind damit komplementär zu einer atomistischen Modellierung. Zur atomistischen Modellierung bieten sich Monte Carlo Methoden oder Molekulardynamik-Simulationen, wie sie auch in dieser Arbeit verwendet werden (siehe auch Kap. 3), an. In einem Kontinuumsmodell werden gewissermaßen die Folgen atomistischer Prozesse auf einer gröberen Skala beschrieben. Die allgemeinen Konzepte der Kontinuumsmodellierung von Oberflächenprozessen sind in der Literatur ausführlich dargestellt (siehe z.B. [BS95; Vic92]).

\subsection{Grundlagen}

Die Oberflächenstruktur wird in einem Kontinuumsmodell durch eine skalare Funktion, die von den Ortskoordinaten einer Bezugsebene und der Zeit abhängig ist, eindeutig beschrieben. Als Bezugsebene dient im Folgenden die Substratebene. Damit kann jedem Ort über der Substratebene $\vec{x}=(x, y)$ zu jedem Zeitpunkt $t$ eine Höheninformation $H$ zugeordnet werden. Somit ist der gesamten Substratfläche eine Höheninformation an allen Orten durch die skalare Funktion $H(\vec{x}, t)$, die im Folgenden auch Höhenfunktion genannt wird, eindeutig zugeordnet. Für eine Modellierung von Oberflächenprozessen ist die Art und Weise von Interesse, in der sich eine gegebene Oberflächenstruktur unter den gegebenen Bedingungen zeitlich entwickelt. Für die mathematische Formulierung der zeitlichen Entwicklung der Höhenfunktion wird ihre Ableitung nach der Zeit $\partial H / \partial t$ verwendet. Der übliche Ansatz für die Darstellung der zeitlichen Ableitung lautet [BS95]:

$$
\frac{\partial H(\vec{x}, t)}{\partial t}=G[H(\vec{x}, t)]+F+\xi(\vec{x}, t) .
$$

Mögliche Beiträge für das Funktional $G$ ergeben sich aus der Höhenfunktion $H$ selbst und deren räumlichen Ableitungen. Das Funktional $G$ entspricht der mathematischen Formulierung der Oberflächenprozesse. $F$ ist die mittlere Wachstums- bzw. Abtragsrate. Durch die Funktion $\xi$ wird berücksichtigt, dass Prozesse wie Schichtwachstum oder auch Ionenbeschuss immer stochastischer Natur sind. Deshalb muss das Modell auch einen Term beinhalten, der ein solches stochastisches Rauschen beschreibt. Gleichung (4.1) lässt sich durch Übergang in ein mitbewegtes Koordinatensystem $h(\vec{x}, t)=H(\vec{x}, t)-F t$ vereinfachen, wenn man des Weiteren berücksichtigt, dass das Funktional $G$ nur von der relativen Höhe $h$ abhängig ist. Damit ergibt sich als Gleichung für die zeitliche 
Entwicklung der relativen Höhe

$$
\frac{\partial h(\vec{x}, t)}{\partial t}=G[h(\vec{x}, t)]+\xi(\vec{x}, t) .
$$

\subsection{Kontinuumsmodellierung und Skalenkonzepte}

Mit Gleichungen des Typs Gl. (4.2) lassen sich verschiedene Strukturbildungsphänomene beschreiben, bei denen insbesondere auch Nichtgleichgewichtsprozesse von Bedeutung sein können. Für Strukturbildungsprozesse hat sich anhand verschiedenster Beispiele gezeigt, dass komplexe Strukturen entstehen können, die nicht glatt sondern von fraktaler Natur sind, so dass für das Beispiel dünner Schichten eine raue Oberfläche nichtganzzahliger Dimension $D_{f}$ mit $2<D_{f}<3$ entstehen kann. Außerdem können die Strukturen zumindest in einer gewissen Näherung als selbstaffin bezeichnet werden [BS95; Vic92]. Dabei heißt selbstaffin, dass durch geeignete Skalierung der Höhenfunktion $h$, der Substratkoordinate $\vec{x}$ und der Zeit $t$ die Form der Strukturen erhalten bleibt. Mathematisch formuliert heißt das, dass sich für die Transformation

$$
\begin{aligned}
\vec{x} & \mapsto b \vec{x} \\
t & \mapsto b^{z} t \\
h & \mapsto b^{\chi} h
\end{aligned}
$$

das Skalenverhalten

$$
h(\vec{x}, t) \propto b^{-\chi} h\left(b \vec{x}, b^{z} t\right)
$$

ergibt. Dabei ist $z$ der dynamische Exponent und $\chi$ der Rauigkeitsexponent. Diese Selbstaffinität kann allerdings nur in gewissen Grenzen gelten, die durch endliche Systemgröße einerseits und endliche Größe der Atome andererseits gegeben sind und sie gilt ohnehin nur im statistischen Sinn aufgrund des statistischen Charakters der beitragenden Prozesse. Das beschriebene Skalenverhalten geht einher mit einem entsprechenden Verhalten der rms-Rauigkeit $\sigma$ (siehe Kap. 2.3.4 zur Definition) als Funktion der Zeit $t$ und der Systemgröße $L$ [BS95]:

$$
\begin{aligned}
& \sigma(L, t=\text { const }) \propto\left\{\begin{array}{l}
L^{\chi} \text { für } L \ll R_{c}(t) \\
\text { const für } L \gg R_{c}(t)
\end{array}\right. \\
& \sigma(L=\text { const, } t) \propto\left\{\begin{array}{l}
t^{\beta} \text { für } t \ll t_{c} \\
\text { const für } t \gg t_{c} .
\end{array}\right.
\end{aligned}
$$

$R_{c}(t)$ und $t_{c}$ sind eine für das betrachtete Systen charakteristische laterale Strukturgröße bzw. Übergangszeit und $\beta$ ist der Wachstumsexponent, der mit den beiden zuvor benannten Exponenten über die Beziehung $z=\chi / \beta$ verbunden ist. Die Gleichungen (4.5) und (4.6) entsprechen der Family-Vicsek-Skalenhypothese [FV85]. Damit zeigt die rms-Rauigkeit in bestimmten Bereichen ein Verhalten, das zum einen durch den 
Rauigkeitsexponenten $\chi$ und zum anderen durch den Wachstumsexponenten $\beta$ charakterisiert ist. Während $\beta$ in der Regel durch die Beziehung (4.6) bestimmt wird, wird für die Bestimmung von $\chi$ oft ausgenutzt, dass für die Höhen-Differenz-Korrelationsfunktion gilt [BS95]:

$$
H(r) \propto r^{2 \chi} \text { für } r \ll R_{c}(t)
$$

Nur für wenige Gleichungen der Art von Gl. (4.2) lassen sich der Wachstumsexponent $\beta$ und der Rauigkeitsexponent $\chi$ analytisch (z.B. mit der Renormierungsgruppentheorie) bestimmen, während in der Mehrzahl der Fälle numerische Lösungsverfahren angewendet werden [BS95]. Über die Werte für die Exponenten lassen sich die zugehörigen Strukturbildungsphänomene in Universalitätsklassen einordnen [BS95].

Die beschriebenen Konzepte sind für das Wachstum amorpher dünner Schichten relevant, so dass die entsprechenden Ergebnisse im Rahmen dieser Konzepte analysiert werden können [BS95]. Beim Ionenbeschuss handelt es sich ebenfalls um einen Strukturbildungsprozess im Nichtgleichgewicht, so dass diese Konzepte auch hier angewendet werden, selbst wenn es sich um einen Glättungs- und nicht um einen Aufrauungsprozess handelt.

\subsection{Kontinuumsmodell im Fourierraum}

Eine Alternative zur direkten Lösung einer Evolutionsgleichung vom Typ der Gleichung (4.2) ergibt sich durch eine Fouriertransformation bezüglich der räumlichen Koordinaten. Dieses Verfahren erweist sich besonders dann als geeignet, wenn das Funktional $G$, das die Oberflächenprozesse beschreibt, im Ortsraum linear bezüglich $h$ ist. Ein solcher linearer Ansatz, der räumliche Ableitungen verschiedener Ordnungen der Höhenfunktion berücksichtigt, lautet [TW94]:

$$
\frac{\partial \hat{h}(\vec{q}, t)}{\partial t}=-\sum_{j} b_{j} q^{j} \hat{h}(\vec{q}, t)+\xi(\vec{q}, t), \quad j \in \mathbb{N} .
$$

Dabei bedeutet` jeweils die Fouriertransformation bezüglich der Ortskoordinaten und $\vec{q}$ ist der Wellenvektor. Schon durch frühere Arbeiten von Mullins und Herring konnten den Termen für $j=1,2,3,4$ Transportprozesse zugeordnet werden [Mul59; Her50]. So liefert viskoses Fließen der gesamten Schicht einen Term der Ordnung $j=1$. Für $j=2$ ist Desorption bzw. Transport durch die Gasphase der von beiden vorgeschlagene Prozess [Her50; Mul59]. Aber auch andere Prozesse gehen mit der Ordnung $j=2$ ein, jedoch mit anderem Vorzeichen im Koeffizienten: Beim Schichtwachstum gibt es den Prozess der Selbstabschattung [Sev73; RML $\left.{ }^{+} 00\right]$ und während der Bestrahlung den Prozess der krümmungsabhängigen Sputtererosion [BH88]. Für $j=3$ kann Volumendiffusion verantwortlich sein und $j=4$ kann durch Oberflächendiffusion bedingt sein [Her50; Mul59] oder auch durch viskoses Fließen in einem begrenzten Oberflächenbereich (siehe Kap. 4.5.4.1). Die Lösung der Gleichung (4.8) ist durch eine Exponentialfunktion gegeben und die Vorzeichen der Koeffizienten $b_{j}$ entscheiden darüber, ob der Term der zugehörigen Ordnung 
zur Glättung oder zur Aufrauung beiträgt. Im deterministischen Fall, also bei Abwesenheit eines Rauschterms, ist die Lösung nämlich gegeben durch

$$
\hat{h}(\vec{q}, t)=\hat{h}(\vec{q}, t=0) \cdot e^{-t \sum_{j} b_{j} q^{j}} .
$$

Dabei beschreibt $\hat{h}(\vec{q}, t=0)$ die Startkonfiguration der Höhenfunktion. Für einen Ansatz der Form aus Gleichung (4.8) lässt sich die in Kap. 2.3.4 definierte spektrale Leistungsdichte wie in [Vil91] berechnen!:

$$
C(q, t)=R(q) \frac{1-\exp \left(-2 t \sum b_{j} q^{j}\right)}{\sum b_{j} q^{j}} .
$$

$R(q)$ ist hier die Rauschstärke, wie sie auch später in Gleichung (4.15) bzw. genauer gesagt durch deren Fouriertransformierte definiert ist. Hier ist in einer etwas allgemeineren Form eine von der q-Mode abhängige Rauschstärke zugelassen. Diese Gleichung gilt, falls die Evolutionsgleichung als Ausgangspunkt eine glatte Oberfläche hat. Wendet man aber eine Evolutionsgleichung auf eine bereits bestehende Struktur an, so ergibt sich für die spektrale Leistungsdichte [MA01]:

$$
C(q, t)=C_{0}(q) \exp \left(-2 t \sum b_{j} q^{j}\right)+R(q) \frac{1-\exp \left(-2 t \sum b_{j} q^{j}\right)}{\sum b_{j} q^{j}} .
$$

Hier ist $C_{0}(q)=C(q, t=0)$ die spektrale Leistungsdichte zu Beginn des betrachteten Prozesses. Im stationären Zustand (Grenzfall $t \rightarrow \infty$ ) ergibt sich aus Gleichung (4.11) das Verhalten [MA01]

$$
\lim _{t \rightarrow \infty} C(q, t)=\frac{R(q)}{\sum b_{j} q^{j}} .
$$

Dieser stationäre Zustand wird im Bereich großer $q$-Werte früher erreicht als für kleine $q$-Werte. Mit Hilfe dieser Formeln lassen sich aus experimentell bestimmten spektralen Leistungsdichten in linearer Näherung Rückschlüsse auf die Transportprozesse ziehen. Im einfachsten Fall kann davon ausgegangen werden, dass das Rauschen räumlich und zeitlich unkorreliert ist, so dass eine Konstante $R$ in die Formeln eingeht, die nicht mehr vom Wellenvektor abhängig ist. Falls nun ein Prozess, der einem festen Wert für $j$ entspricht, dominiert, äußert sich das in einem Zerfall von $C(q)$, der proportional zu $q^{-j}$ ist. Deswegen werden die spektralen Leistungsdichten in der Regel in doppeltlogarithmischer Auftragung präsentiert, in der sich dann ein linearer Abfall mit der entsprechenden Steigung ergibt.

Weiterhin zeigen die spektralen Leistungsdichten ein Maximum, wenn die zugrundeliegende Oberflächenstruktur durch eine bevorzugte laterale Strukturgröße ausgezeichnet ist.

\subsection{Schichtwachstum}

Für das im Rahmen dieser Arbeit experimentell bearbeitete System $\mathrm{Zr}_{65} \mathrm{Al}_{7.5} \mathrm{Cu}_{27.5}$ ist die Modellierung des Wachstums dünner Filme auf der Basis einer Gleichung des Typs

\footnotetext{
${ }^{1}$ In der zitierten Arbeit fehlt allerdings ein Faktor 1/2.
} 
Gl. (4.1) bereits in der Vergangenheit ausführlich untersucht worden. Dabei konnte gezeigt werden, dass sich die Oberflächenstruktur während des Wachstums zumindest für dünne und mittlere Schichtdicken, also für Schichtdicken bis zu $500 \mathrm{~nm}$, in sehr guter Übereinstimmung mit der folgenden Gleichung entwickelt [May97; MMS99; Rai00; $\mathrm{RML}^{+}$00; SSM02; Str04]:

$$
\frac{\partial h}{\partial t}=a_{1} \nabla^{2} h+a_{2} \nabla^{4} h+a_{3} \nabla^{2}(\nabla h)^{2}+a_{4}(\nabla h)^{2}+\xi
$$

Zur Vereinfachung sind hier die Funktionen $h$ und $\xi$ ohne ihre Argumente aufgeschrieben. Während des Wachstums wird die statistische Natur des Depositionsprozesses durch weißes, d.h. räumlich und zeitlich unkorreliertes, Rauschen mit dem Mittelwert null beschrieben. Es gilt also

$$
\begin{gathered}
\langle\xi(\vec{x}, t)\rangle=0 \\
\left\langle\xi(\vec{x}, t) \xi\left(\vec{x}^{\prime}, t^{\prime}\right)\right\rangle=2 R \delta^{(2)}\left(\vec{x}-\vec{x}^{\prime}\right) \delta\left(t-t^{\prime}\right) .
\end{gathered}
$$

Dabei bezeichnet $R$ die Rauschstärke, die in diesem Fall der Forderung nach unkorreliertem Rauschen entsprechend als konstant angenommen wird. Gleichung (4.13) wird in dieser Arbeit verwendet, um die Struktur der gewachsenen $\mathrm{Zr}_{65} \mathrm{Al}_{7.5} \mathrm{Cu}_{27.5}$ Schicht $\mathrm{zu}$ erzeugen, die dann als Ausgangspunkt für die Modellierung der Ionenbestrahlung genutzt werden kann. Für die Wachstumsgleichung konnte gezeigt werden, dass sämtliche verwendeten Terme notwendig und ausreichend sind, um die experimentellen Ergebnisse beschreiben zu können $\left[\mathrm{RML}^{+} 00\right]$. Weiterhin sind sämtliche Koeffizienten $a_{j}$ sowie die Rauschstärke $R$ quantitativ bekannt $\left[\mathrm{RML}^{+} 00\right]$ und es ist jedem Term in der Gleichung ein physikalischer Prozess zugeordnet. Die wesentlichen Ergebnisse aus den zitierten Arbeiten werden im folgenden Abschnitt kurz wiedergegeben.

Der $a_{1}$-Term beschreibt den Effekt einer geometrischen Selbstabschattung. Dabei führen lokale anziehende Kräfte auf die neu ankommenden Atome dazu, dass auf den Hügeln die Auftreffdichte ankommender Atome größer ist als in den Tälern. Daher gilt $a_{1}<0$ und der Term trägt zur Aufrauung bei. Der $a_{2}$-Term beschreibt eine krümmungsinduzierte Oberflächendiffusion von Adatomen mit dem chemischen Potential als Triebkraft. Dieser Term wirkt somit glättend und es gilt $a_{2}<0$. Das Zusammenspiel dieser beiden Terme verursacht eine Instabilität, die zum verstärkten Wachstum einer ausgezeichneten Struktur der Mode $q_{c}=\sqrt{a_{1} /\left(2 a_{2}\right)}$ führt [RML $\left.{ }^{+} 00\right]$. Der $a_{3}$-Term beruht darauf, dass die Auftreffrate von Atomen pro Einheitsfläche auf der Probenoberfläche von der lokalen Steigung der Oberfläche abhängig ist. Auf den Flanken ist aus geometrischen Gründen die Auftreffdichte kleiner als an den Orten verschwindender Steigung. Ein durch die Entropie getriebener Konzentrationsausgleich zwischen den Adatomen ist dann mit einem Partikelstrom verbunden, der von den Orten betragsmäßig geringer Steigung zu denen mit betragsmäßig großer Steigung verläuft. Der $a_{4}$-Term beschreibt ein nichtlineares Überwachsen, das sich aufgrund kleinerer Teilchendichten unterhalb eines auf einer Flanke ankommenden Atoms ergibt. 


\subsection{Modifikation der Oberflächenstruktur durch lonenbestrahlung}

In diesem Abschnitt sollen Terme im Kontinuumsmodell erläutert werden, die während der Bestrahlung einer Oberfläche mit Ionen relevant sein können. Diese Terme lassen sich in aufrauende und glättende einteilen. Um im weiteren Verlauf der Arbeit quantitative Aussagen machen zu können, sollen jeweils die vollständigen Terme inklusive der durch physikalische Größen beschriebenen Koeffizienten angegeben werden. Weil zunächst die grundlegenden Effekte untersucht werden sollen, werden die jeweiligen Terme in linearer Näherung behandelt.

\subsubsection{Sputtererosion}

Wenn energetische Ionen auf eine Oberfläche treffen, ist ein möglicher Prozess, dass Atome aufgrund von Impuls- und Energieübertrag aus der Oberfläche herausgeschlagen werden. Dieser Prozess wird als Sputtererosion ${ }^{2}$ bezeichnet. Die Anzahl der pro eingeschossenem Atom herausgeschlagenen Atome nennt man die mittlere Sputterausbeute $Y$ (sputter yield). Sie ist vom Einfallswinkel, der Energie der auftreffenden Atome und der Atomsorte der Ionen und der Schicht abhängig. Der Einfluss dieser Sputtererosion auf die Oberflächenstruktur wurde bereits im Zusammenhang mit der unter bestimmten Bedingungen auftretenden Ripplebildung ${ }^{3}$ von Bradley und Harper untersucht [BH88]. Dabei hat sich gezeigt, dass die Anzahl der pro Fläche erodierten Atome von der lokalen Krümmung der Oberfläche abhängig ist. Genauer gesagt gilt für den Beitrag dieses Prozesses in der Näherung kleiner Steigungen $(|\nabla h| \ll 1)$ und für den hier betrachteten Fall des Beschusses parallel zur Substratnormalen in einem Koordinatensystem, in dem die mittlere Abtragsrate heraustransformiert ist [BH88]:

$$
\frac{\partial h}{\partial t}=-\frac{F a_{E} Y}{2 n} \nabla^{2} h
$$

Dabei ist $F$ der Fluss der Ionen, $a_{E}$ die durchschnittliche Tiefe der Energiedeposition, $n$ die atomare Dichte und $Y$ die mittlere Sputterausbeute. Zugehörige stochastische Beiträge werden in Abschnitt 4.5.6 erläutert. Somit werden in den Tälern $\left(\nabla^{2} h>0\right)$ Atome mit einer höheren Rate abgetragen als auf den Hügeln $\left(\nabla^{2} h<0\right)$ und das führt zu einer Aufrauung der Oberfläche.

Die Fouriertransformation bezüglich der Ortskoordinaten liefert wegen $F T\left[\nabla^{2} h\right]=$ $(-i \vec{q})^{2} \hat{h}=-q^{2} \hat{h}$ die Evolutionsgleichung

$$
\frac{\partial \hat{h}}{\partial t}=\frac{F a_{E} Y}{2 n} q^{2} \hat{h}
$$




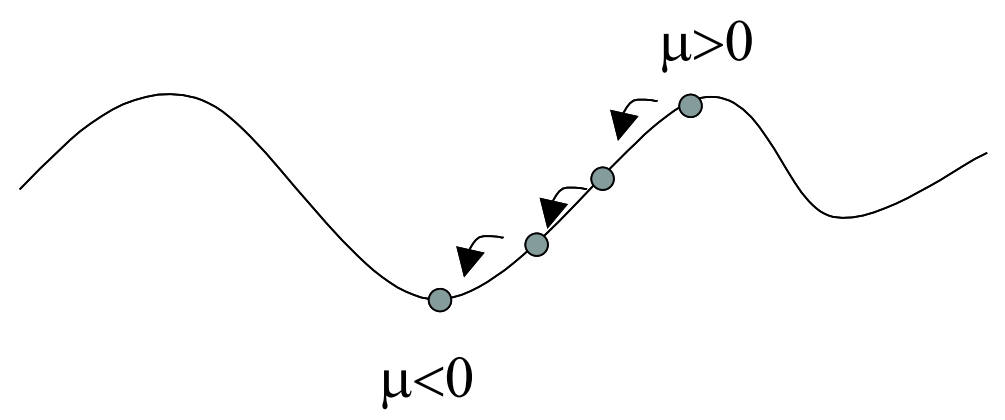

Abbildung 4.1: Diffusive Bewegung von Oberflächenatomen.

\subsubsection{Oberflächendiffusion}

Nach dem Einschuss eines Ions geraten viele Atome in Bewegung, so dass anzunehmen ist, dass sie sich danach in einer bezüglich der freien Energie des Systems möglichst günstigen Konfiguration anordnen. Um den Übergang von einer zufälligen zu einer energetisch günstigen Position zu ermöglichen, ist ein Transportmechanismus nötig. Eine Möglichkeit für einen solchen Transportmechanismus ist die Diffusion auf der Oberfläche. Der Einfluss thermisch aktivierter Diffusion auf die zeitliche Entwicklung von Oberflächenstrukturen wurde bereits von Mullins untersucht [Mul57; Mul59]. Der zugrundeliegende Gedanke dabei ist, dass das chemische Potential für Oberflächenatome proportional zur lokalen Krümmung der Oberfläche ist, wobei das Vorzeichen entsprechend gewählt werden muss, so dass das Potential in den Tälern kleiner ist als auf den Hügeln, weil ein Oberflächenatom in einem Tal aus geometrischen Gründen mehr Nachbarn als auf einem Hügel hat. Dies ist im Einklang mit der Gibb'schen Behandlung des chemischen Potentials an einer gekrümmten Oberfläche. Weiterhin ergibt sich wie in Abb. 4.1 skizziert ein Teilchenstrom, der dem Gradienten des chemischen Potentials proportional ist und wegen der Kontinuitätsgleichung, die wegen der Erhaltung der Teilchenzahl gilt, ergibt sich die lokale Höhenänderung aus der Divergenz des Teilchenstroms. In [Mul57] wird die Rate der Höhenänderung für Strukturen, die nur von einer 1-dimensionalen lateralen Koordinate abhängig sind, berechnet. Es zeigt sich aber, dass für den hier relevanten Fall von Strukturen, die von einer 2-dimensionalen lateralen Koordinate abhängig sind, eine völlig analoge Beziehung gilt. Dort gilt für das chemische Potential $\mu$ zunächst, wenn man das Vorzeichen so wählt, dass auf einem Hügel (im Tal) die Krümmung negativ (positiv) ist [Hud98]

$$
\mu(K)=-2 \gamma \Omega K \text {. }
$$

Dabei ist $\Omega$ das atomare Volumen, $\gamma$ die freie Oberflächenenergie und $K$ die lokale Krümmung der Oberfläche. Auf einer 2-dimensionalen Oberfläche gilt dabei für die mittlere Krümmung bei kleinen Steigungen $(|\nabla h| \ll 1)$ näherungsweise [BSMM97]:

$$
K=\frac{1}{2} \nabla^{2} h .
$$

\footnotetext{
${ }^{2}$ Englisch: to sputter $=$ zerstäuben

${ }^{3}$ Englisch: ripple $=$ Welle
} 
Somit ergibt sich durch Einsetzen in Gleichung (4.18):

$$
\mu=-\gamma \Omega \nabla^{2} h \text {. }
$$

Einsetzen dieser Gleichung in die Beziehung zwischen Teilchenstrom und chemischem Potential und anschließend in die Beziehung zwischen Teilchenstrom und Rate der Höhenänderung wie in [Mul57] liefert für den hier relevanten Fall von Oberflächenstrukturen, die von einer 2-dimensionalen Substratkoordinate abhängig sind:

$$
\frac{\partial h}{\partial t}=-\frac{D_{s} \gamma \Omega^{2} \nu}{k_{B} T} \nabla^{4} h
$$

In dieser Formel repräsentiert $D_{s}$ den Oberflächendiffusionskoeffizienten, $\nu$ die Oberflächendichte der an der Diffusion beteiligten Atome, $k_{B}$ die Boltzmannkonstante und $T$ die Temperatur. Während in [Mul57] $D_{s}$ sich allein aus thermisch aktivierter Diffusion ergibt, beinhaltet $D_{s}$ im hier behandelten Fall die Summe aller zur Diffusion beitragenden Effekte und skaliert daher mit dem Ionenfluss $F$. Diese Rate der Höhenänderung trägt zur Glättung der Oberfläche bei, weil der Ursprung dieser Formel die Tatsache ist, dass sich die Atome von den Orten eines hohen chemischen Potentials (den Hügeln) zu denen eines niedrigen chemischen Potentials (den Tälern) bewegen.

Ein Oberflächendiffusionsmechanismus dieser Art wird auch beim Wachstum der amorphen Schichten angenommen. Dort stellen die während der Deposition neu ankommenden Atome die an einem möglichen Diffusionsprozess beteiligten Atome dar [RML $\left.{ }^{+} 00\right]$. Durch Bestrahlung ist es ebenfalls möglich, Atome an der Oberfläche anzuregen, d.h. Adatome zu erzeugen, die im Folgenden diffundieren können.

Hier liefert die Fouriertransformation bezüglich der Ortskoordinaten wegen $F T\left[\nabla^{4} h\right]=$ $(-i \vec{q})^{4} \hat{h}=q^{4} \hat{h}$ die Evolutionsgleichung

$$
\frac{\partial \hat{h}}{\partial t}=-\frac{D_{s} \gamma \Omega^{2} \nu}{k_{B} T} q^{4} \hat{h} .
$$

\subsubsection{Ballistischer Transport}

Wenn ein Ion auf die Oberfläche trifft, ergibt sich zunächst eine Stoßkaskade, d.h. ballistische Prozesse dominieren [AR98]. Trifft das Ion parallel zur lokalen Oberflächennormalen auf, so gibt es für die Stoßkaskade keine Vorzugsrichtung entlang der Oberflächenkontur. Die Situation ändert sich, wenn das Ion nicht parallel zur lokalen Oberflächennormalen auftrifft. Dieser Fall ist beim Beschuss parallel zur Substratnormalen relevant, wenn die Oberfläche eine gewisse Rauigkeit zeigt und das Ion auf einer Flanke eines Hügels auftrifft. Für die ballistische Stoßkaskade gibt es dann eine Vorzugsrichtung der Art, dass die Atome der Oberfläche bevorzugt von der Flanke ins Tal geschoben werden, wie es in Abb. 4.2 dargestellt ist. Dieser Effekt wurde von Moseler et al vorgeschlagen und seine Relevanz wurde bei sehr niedrigen Bestrahlungsenergien $(E \leq 100 \mathrm{eV})$ für kovalent gebundene Systeme durch MD-Simulationen gezeigt $\left[\mathrm{MGC}^{+}\right.$05]. Dass die Atome von Flanken ins Tal geschoben werden bedeutet, dass ein Strom proportional (und entgegengesetzt) zum Gradienten des Höhenprofils entsteht. Die Rate der Höhenänderung ergibt 


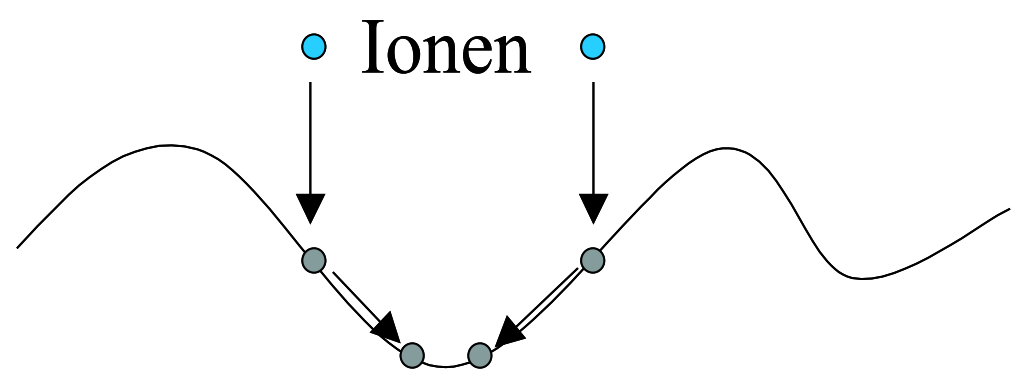

Abbildung 4.2: Ballistischer Glättungsprozess.

sich wiederum wegen der Erhaltung der Anzahl von Atomen und der dementsprechend geltenden Kontinuitätsgleichung als negative Divergenz dieses Partikelstroms. Damit ergibt sich insgesamt $\partial h / \partial t \propto \nabla^{2} h$. Die quantitative Analyse in $\left[\mathrm{MGC}^{+} 05\right]$ liefert:

$$
\frac{\partial h}{\partial t}=F \Omega\left|\frac{\delta}{\tan (\alpha)}\right| \nabla^{2} h
$$

Dabei ist $F$ der Fluss ankommender Ionen, $\Omega$ das atomare Volumen, $\delta$ die Summe der auf die Substratebene projizierten atomaren Verschiebungen pro Ion und $\alpha$ charakterisiert den Betrag der lokalen Steigung der Oberfläche gemäß tan $(\alpha)=|\nabla h|$. In $\left[\mathrm{MGC}^{+} 05\right]$ hat sich gezeigt, dass der Ausdruck $\delta / \tan (\alpha)$ eine von der lokalen Steigung, also von $\alpha$, unabhängige Konstante ist, die allerdings von der Einschussenergie abhängig ist. Dass der Ausdruck $\delta / \tan (\alpha)$ unabhängig von der Oberflächenmorphologie ist, ist auch notwendig, damit in Gleichung (4.23) auf der rechten Seite die gesamte Morphologieabhängigkeit im Term $\nabla^{2} h$ enthalten ist. Gleichung (4.23) beschreibt einen glättenden Prozess, weil die Wachstumsrate auf den Hügeln kleiner als in den Tälern ist.

Die Fouriertransformation bezüglich der Ortskoordinaten liefert wegen $F T\left[\nabla^{2} h\right]=$ $(-i \vec{q})^{2} \hat{h}=-q^{2} \hat{h}$ die Evolutionsgleichung

$$
\frac{\partial \hat{h}}{\partial t}=-F \Omega\left|\frac{\delta}{\tan (\alpha)}\right| q^{2} \hat{h} .
$$

\subsubsection{Viskoses Fließen}

Durch das Auftreffen eines energetischen Ions können sich an der Oberfläche kleine Bereiche lokal viskos deformieren [SK56; AR98]. Die Ausdehnung und Lebensdauer dieser aufgeschmolzenen Bereiche (,thermal spikes“) hängt von der Energie und Masse der eingeschossenen Ionen und natürlich vom der Bestrahlung ausgesetzten Material ab. Ein flüssiger Bereich an der Oberfläche sollte sich in einer Weise anordnen, die dazu führt, dass die freie Oberflächenenergie minimiert wird. Das wird erreicht, wenn die Oberfläche auf einer Größenskala oberhalb der Atomgrößen glatt ist. Hügel sollten also zerfließen und die Täler auffüllen, wenn die gesamte Oberfläche flüssig ist.

Nun gilt es zu klären, wie ein solches Fließen die Evolution der Oberflächenmorphologie beeinflusst. Die allgemeine Lösung der Frage, wie eine inkompressible, zähe Flüssigkeit 
a.)

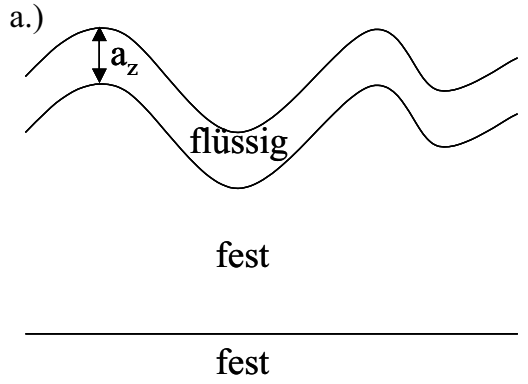

b.)

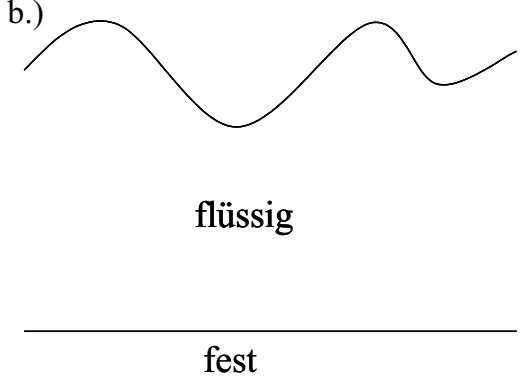

Abbildung 4.3: Viskoses Fließen a.) in einer dünnen Oberflächenschicht, b.) in einem ausgedehnten Volumenbereich

unter gegebenen Randbedingungen fließt, ist in der Hydrodynamik durch die NavierStokes-Gleichung gegeben [LL66]. Die Navier-Stokes-Gleichung lässt sich unter der Annahme einer kleinen Reynolds-Zahl, was der Annahme entspricht, dass die durch die Viskosität bedingte Reibungskraft sehr viel größer als die durch die Masse bedingte Kraft ist [Pai56], vereinfachen und führt im Ergebnis zur Stokes-Gleichung [LL66]. Diese Annahmen sind im zu betrachtenden Fall erfüllt. Für den $1+1$ dimensionalen Fall (eine laterale und eine vertikale Dimension) wurde von Orchard [Orc62] die StokesGleichung unter den Randbedingungen, dass an der Grenzfläche flüssig - fest keine Bewegung stattfindet und an der Grenzfläche flüssig - Vakuum keine Scherkräfte senkrecht zur Oberflächennormalen wirken, im Fourierraum (Fouriertransformation bezüglich der lateralen Ortskoordinate) gelöst. Als Ergebnis für die Rate der Höhenänderung ergeben sich zwei Grenzfälle, die sich anhand des Verhältnisses der Dicke der flüssigen Schicht zur typischen lateralen Strukturgröße unterscheiden. Es muss also unterschieden werden zwischen einem Oberflächenfließen in einer dünnen flüssigen Schicht an der Oberfläche und einem Volumenfließen, bei dem die Dicke der flüssigen Schicht größer ist als die Strukturgröße. Zwischen diesen beiden Grenzfällen, die in Abb. 4.3 skizziert sind, gibt es einen kontinuierlichen Übergang [Orc62].

\subsubsection{Viskoses Oberflächenfließen}

Für den Grenzfall der dünnen flüssigen Schicht an der Oberfläche ergibt sich als Rate der Höhenänderung im Fourierraum [Orc62]:

$$
\frac{\partial \hat{h}}{\partial t}=-\frac{\gamma a_{z}^{3}}{3 \eta} q^{4} \hat{h} .
$$

Dabei ist $\gamma$ die freie Oberflächenenergie, die in einer Flüssigkeit der Oberflächenspannung gleich ist, $a_{z}$ die Dicke der flüssigen Schicht und $\eta$ die Viskosität. Es lässt sich aber auch die entsprechende Gleichung im Ortsraum herleiten, wie es in [SL88] für 1+1 Dimensionen durchgeführt ist. Diese Herleitung lässt sich leicht auf 2+1 Dimensionen übertragen und liefert in einer linearisierten Approximation (siehe Anhang 11.1)

$$
\frac{\partial h}{\partial t}=-\frac{\gamma a_{z}^{3}}{3 \eta} \nabla^{4} h
$$


Während in den zitierten Arbeiten die Viskosität stets durch thermische Prozesse bestimmt ist, beinhaltet sie in dieser Arbeit auch die strahlungsinduzierten Eigenschaften und skaliert daher mit dem Ionenfluss $F$.

Die Fouriertransformation der Gleichung (4.26) liefert wieder Gleichung (4.25), so dass damit gezeigt ist, dass Gleichung (4.25) auch für 2+1 Dimensionen gilt.

Damit zeigt der Transportmechanismus des viskosen Oberflächenfließens dieselbe Morphologieabhängigkeit wie der Prozess der Oberflächendiffusion, allerdings mit unterschiedlichen Proportionalitätskonstanten.

\subsubsection{Viskoses Volumenfließen}

Für den Grenzfall einer Dicke der Flüssigkeitsschicht, die sehr viel größer als die typische Strukturgröße ist, ergibt sich im Fourierraum nach Mullins [Mul59; Orc62]:

$$
\frac{\partial \hat{h}}{\partial t}=-\frac{\gamma}{2 \eta} q \hat{h}
$$

Wiederum beruht die Viskosität in den zitierten Arbeiten auf thermischen Prozessen, aber im hier behandelten Fall sind die strahlungsinduzierten Prozesse enthalten.

Der Grund dafür, dass Volumenfließen eine andere Auswirkung auf die Morphologieentwicklung als Oberflächenfließen hat, liegt in der unterschiedlichen Richtung des Teilchentransports im Bereich der Oberfläche. Beim Volumenfließen liegt die Hauptkomponente der Teilchenbewegung nämlich parallel zur Substratnormalen, während sie beim Oberflächenfließen senkrecht darauf steht [Orc62].

Gleichung (4.27) lässt sich im Ortsraum nicht direkt durch eine räumliche Ableitung erster Ordnung einer reellwertigen Funktion $h$ auf der rechten Seite der Gleichung darstellen, da räumliche Ableitungen ungerader Ordnung bei der Fouriertransformation einen Faktor $i$ verursachen ( $i=$ imaginäre Einheit). Es gibt allerdings den Vorschlag, den Prozess des Volumenfließens durch einen Ausdruck der Form

$$
\frac{\partial h}{\partial t}=-\frac{\gamma}{2 \eta}|\nabla h|
$$

zu beschreiben [Car98], wobei hier wie schon bisher $\eta$ die strahlungsinduzierte Viskosität, die mit dem Ionenfluss skaliert, ist. Die Gleichungen (4.27) und (4.28) lassen sich aber nicht durch Fouriertransformation ineinander überführen. Dass sich beide Gleichungen nicht entsprechen wird auch noch auf eine andere Art und Weise klar. Gleichung (4.27) besagt, dass die Wachstumsgeschwindigkeit auf den Hügeln minimal und in den Tälern maximal ist [Mul59]. Gleichung (4.28) besagt dagegen, dass die Wachstumsgeschwindigkeit auf den Hügeln und in den Tälern null ist und auf den Flanken maximal wird. Damit beschreibt Gleichung (4.27) das für ein Zerfließen der Strukturen erwartete Verhalten und (4.28) nicht. Gegen die Beschreibung des Fließens durch Gleichung (4.28) spricht ebenfalls die Tatsache, dass diese Gleichung die Teilchenzahl nicht erhält, weil nach ihr für die Wachstumsgeschwindigkeit stets $\partial h / \partial t \leq 0$ gilt. Gleichung (4.27) erhält dagegen die Teilchenzahl, wenn $\langle h\rangle_{\vec{x}}=0$ gilt, weil Wachstumsgeschwindigkeit und relative Höhenfunktion zueinander antiproportional sind. Für einen Glättungsprozess durch 
Fließen gibt es keinen Grund, die Forderung nach Erhaltung der Teilchenzahl aufzuheben.

\subsubsection{Nichtlokale Prozesse}

Die bisher besprochenen Prozesse, die sich durch eine räumliche Ableitung der Höhenfunktion modellieren lassen, kann man insofern als lokale Prozesse bezeichnen, weil die Rate der Höhenänderung an einem Ort sich alleine aus Eigenschaften an diesem Ort selbst beschreiben lässt. Diese Eigenschaft trifft auf alle diskutierten Prozesse außer dem Volumenfließen zu. Den beschriebenen lokalen Prozessen ist gemein, dass die Höhenänderung durch einen Partikelstrom bedingt ist, der entlang der lokalen Oberfläche gerichtet ist (also senkrecht zur Normalen). Im Unterschied dazu beruht die Höhenänderung durch Volumenfließen auf einem Partikelstrom, der senkrecht zur Oberfläche (also entlang der Normalen) gerichtet ist. Deshalb ist zu erwarten, dass beim Volumenfließen die Höhenänderung nicht alleine aus den geometrischen Eigenschaften am selben Ort bestimmt werden kann. Es handelt sich damit um einen nichtlokalen Prozess, wie es auch in [UHC01] diskutiert wird.

Als ein nichtlokaler Term zur Beschreibung von Oberflächenprozessen während der Ionenbestrahlung wird in der Literatur ein dissipativer Term der Form

$$
\frac{\partial h}{\partial t}=-\kappa\left(h-\langle h\rangle_{A}\right)
$$

diskutiert, wobei $A$ ein Teil der Oberfläche, über den räumlich gemittelt wird, und $\kappa$ eine positive Konstante, die bisher noch nicht mit physikalischen Größen identifiziert werden konnte, ist $\left[\mathrm{FBS}^{+} 04\right]$. In der zitierten Arbeit wird $A$ allerdings zunächst der gesamten Fläche gleichgesetzt, ohne dies näher zu begründen. Dieser Dämpfungsterm wird mit einem Sputter-Redepositions-Prozess in Verbindung gebracht, ohne dass diese Verbindung dort bewiesen werden kann. Damit ist gemeint, dass auf einer sehr rauen Oberfläche ein von einem Hügel gesputtertes Atom zu einem anderen Hügel fliegt und sich dort festsetzt. Auch die weiterführende Analyse dieses Terms konnte diesen Zusammenhang jedoch nicht eindeutig herstellen [VL05].

Führt man eine Fouriertransformation bezüglich der Ortskoordinaten durch, so erhält man in dem Koordinatensystem, in $\operatorname{dem}\langle h\rangle_{A}=0$ gilt:

$$
\frac{\partial \hat{h}}{\partial t}=-\kappa \hat{h} .
$$

Damit entspräche dieser Prozess im linearen Modell gemäß Gleichung (4.8) einem Prozess der Ordnung $j=0$. Insbesondere beschreibt der dissipative Term also nicht das Volumenfließen im Sinne von Gleichung (4.27). Die tatsächliche physikalische Bedeutung dieses Terms ist bisher noch nicht eindeutig geklärt.

\subsubsection{Stochastisches Rauschen}

Der Ionenbeschuss ist stochastischer Natur, denn der Ort, an dem ein Ion die Oberfläche trifft, ist durch den Zufall bestimmt. Deshalb muss ein Term zur Morphologieentwicklung 
beitragen, der den stochastischen Charakter wiedergibt. Da das Auftreffen eines Ions in Raum und Zeit der gleichen Verteilung gehorchen sollte wie das Auftreffen von Atomen beim Schichtwachstum, soll beim Ionenbeschuss in Analogie zum Schichtwachstum ein in Raum und Zeit unkorreliertes Rauschen angenommen werden, wie es durch die Gleichungen (4.14) und (4.15) beschrieben wird, wobei hier die Rauschstärke $R$ eine andere Bedeutung hat. Gemäß den Ergebnissen zum Ionenbeschuss im MeV-Energiebereich nach S. G. Mayr [MA01] ist sie, wenn zunächst wie bisher nur das Rauschen auf atomarer Skala berücksichtigt wird, gegeben durch

$$
2 R=F Y \Omega^{2}
$$

mit dem Fluss $F$, der Sputterausbeute $Y$ und dem atomaren Volumen $\Omega$. Bei Bestrahlungsenergien im MeV-Bereich hat sich in der Arbeit von S. G. Mayr [MA01] gezeigt, dass dem Rauschen auf atomarer Skala ein weiterer Rauschprozess überlagert ist, der eine Aufrauung auf größerer Skala hervorruft. Die beiden Prozesse sind statistisch unabhängig voneinander und daher additiv. Ob der zweite Prozess auch bei dem in der vorliegenden Arbeit behandelten keV-Energiebereich relevant ist, wird im weiteren Verlauf geklärt. Bereits in [MA01] wird aber klar, dass das Gewicht des zweiten Prozesses mit kleiner werdender lateraler Ausdehnung der „thermal spikes“ abnimmt. Deshalb sollte seine Bedeutung bei den hier untersuchten kleineren Energien wesentlich kleiner sein und Gleichung (4.31) kann als erste Näherung verwendet werden.

\subsection{Relatives Gewicht der Prozesse}

Ein Ziel bei der Untersuchung von Oberflächenmodifikationen ist die Abschätzung des relativen Beitrags der beteiligten Prozesse zum Gesamtergebnis. In einem linearen Modell äußert sich im deterministischen Fall das Gewicht der Einzelprozesse direkt in der durch Gleichung (4.9) gegebenen Lösung der Evolutionsgleichung. Der Verlauf der Lösung zeigt, dass für jeden glättenden Prozess $j$ die charakteristische Abklingzeit, nach der die Amplitude einer ursprünglichen Oberflächenstruktur auf des 1/e-fache des Ursprungswertes abgefallen ist, durch den Ausdruck $\left(b_{j} q^{j}\right)^{-1}$ gegeben ist. Ein Maß für das Gewicht oder die Stärke eines Glättungsprozesses ist gerade das Inverse der Abklingzeit, also für jeden Prozess der Ausdruck $b_{j} q^{j}$. Das relative Gewicht verschiedener glättender Prozesse der Ordnung $m$ und $n$ zueinander lässt sich demnach durch Auswertung des entsprechenden Quotienten $b_{m} q^{m} / b_{n} q^{n}$ berechnen.

\subsection{Einfluss der Glättungsprozesse auf die Entwicklung der Rauigkeit}

Der Einfluss der beteiligten Prozesse auf die spektralen Leistungsdichten $C(q, t)$ ist in Kap. 4.3 bereits diskutiert. Dort wird die räumliche Information zu einem festen Zeitpunkt analysiert. Hier soll nun untersucht werden, wie sich die Rauigkeit, die eine 
räumlich gemittelte Größe darstellt, als Funktion der Zeit entwickelt. Dabei werden wiederum die linearen Prozesse berücksichtigt und im Fourierraum behandelt.

Die rms-Rauigkeit lässt sich darstellen als [BS95]

$$
\sigma^{2}(t)=\int_{\mathbb{R}^{2}}|\hat{h}(\vec{q}, t)|^{2} d \vec{q}
$$

was im Falle der wegen der senkrechten Bestrahlung vorhanden Isotropie auf der Oberfläche direkt auf den Zusammenhang

$$
\sigma^{2}(t)=2 \pi \int_{0}^{\infty} q|\hat{h}(q, t)|^{2} d q
$$

führt. Wegen $C(q, t)=|\hat{h}(q, t)|^{2}$ kann man nun die bekannte Form der spektralen Leistungsdichte gemäß Gleichung (4.11) einsetzen. Um zu analytischen Lösungen zu kommen, ist aber die Betrachtung der existierenden Grenzfälle für die als Ausgangspunkt verwendete Oberfläche, die durch die spektrale Leistungsdichte $C_{0}(q)$ beschrieben wird, sinnvoll. Für die Beschreibung der Prozesse während der Bestrahlung wird die Lösung des linearen Modells aus Gleichung (4.9) verwendet, wobei hier der stochastische Beitrag vernachlässigt wird. Für die folgenden Grenzfälle ergeben sich analytische Lösungen:

1. Nur ein Wellenvektor $q_{s}$ trägt zur spektralen Leistungsdichte der ursprünglichen Struktur bei; es gilt also $C_{0}(q)=\delta\left(q-q_{s}\right)$. Das bedeutet, dass während des Schichtwachstums eine Mode stark dominiert hat. Dann ergibt sich für eine Überlagerung von Prozessen der Ordnung $j \geq 0$ :

$$
\sigma^{2}(t)=2 \pi \int_{0}^{\infty} q|\hat{h}(q, 0)|^{2} e^{-2 t \sum b_{j} q^{j}} d q=2 \pi q_{s} e^{-2 t \sum b_{j} q_{s}^{j}} \Longrightarrow \sigma(t) \propto e^{- \text {const } \cdot t} .
$$

In diesem Fall schrumpft die Rauigkeit für jede Überlagerung glättender Prozesse der Ordnung $j$ ebenso wie die Höhenfunktion selbst exponentiell.

2. Die durch das Schichtwachstum verursachte spektrale Leistungsdichte ist vom Wellenvektor unabhängig, d.h. $C_{0}(q)=$ const $=: C$. Das entspricht dem Grenzfall, dass alle Moden während des Schichtwachstums in gleicher Stärke beteiligt sind. Dieser Grenzfall ist gegeben, wenn die Strukturbildung beim Wachstum alleine durch unkorreliertes Rauschen verursacht wird. Dann ergibt sich für jeden Glättungsprozess der Ordnung $j$ :

$$
\sigma^{2}(t)=2 \pi C \int_{0}^{\infty} q e^{-2 b_{j} q^{j} t} d q
$$

Für Prozesse der Ordnung $j \geq 1$ ergibt sich daraus (siehe Anhang 11.2)

$$
\sigma(t) \propto t^{-1 / j}
$$

und für einen Prozess der Ordnung $j=0$ ergibt sich (siehe Anhang 11.2)

$$
\sigma(t) \propto e^{-b_{0} t} .
$$


Damit sind die beiden Extremfälle behandelt, indem in der Ausgangsstruktur zum einen eine Mode dominiert oder zum anderen alle Moden gleichermaßen beteiligt sind. Tatsächlich gilt für die sich aus dem Schichtwachstum ergebende spektrale Leistungsdichte der Zusammenhang aus Gleichung (4.10) mit den sich aus der Wachstumsgleichung (4.13) ergebenden Koeffizienten. Die vollständigen Ausdrücke erschweren aber die Aufgabe, einen analytischen Ausdruck für den Verlauf von $\sigma(t)$ zu erzielen.

\subsection{Numerische Lösung der Kontinuumsmodelle}

Im mathematischen Sinn sind die bisher besprochenen Kontinuumsmodelle durch stochastische partielle Differentialgleichungen beschrieben. Analytische Aussagen sind für solche Gleichungen nur in begrenztem Maße möglich, so dass für die vollständige Lösung auf numerische Lösungskonzepte zurückgegriffen werden muss.

Die in Kap. 4.4 zitierte Gleichung zur Beschreibung des Wachstums von dünnen, gläsernen ZrAlCu Filmen wurde von S. G. Mayr numerisch gelöst [May97; May00] und der dort in $\mathrm{C}++$ entwickelte Computercode wird auch in dieser Arbeit verwendet.

Die verwendete Lösungsmethode ist die Methode der finiten Differenzen. Dabei werden die 2-dimensionalen lateralen Ortskoordinaten und die Zeit diskretisiert. Die Randbedingungen in beiden lateralen Richtungen sind periodisch gewählt. Die numerische Integration der diskretisierten Gleichung erfolgt gemäß dem Euler-Verfahren, bei dem es sich im Prinzip um eine Taylor-Entwicklung handelt [PTVF94]. Das Euler-Verfahren ist ein explizites Lösungsverfahren, bei dem die Genauigkeit in der Zeit von erster Ordnung und die Genauigkeit im Ort mit den verwendeten Ausdrücken der diskretisierten räumlichen Ableitungen von zweiter Ordnung ist. Um eine numerisch stabile Lösung zu erhalten, muss der Quotient aus der Schrittweite bezüglich der Zeit und der bezüglich des Ortes $\Delta t / \Delta x$ stets hinreichend klein gewählt werden. Die für den Rauschterm benötigten Zufallszahlen werden in zwei Schritten erzeugt. Um Pseudozufallszahlen guter Qualität zu erhalten, die gleichverteilt zwischen null und eins liegen, wird der ran2 Zufallszahlengenerator aus [PTVF94], der in [Vau02] implementiert wurde, verwendet. Mit der Polar-Marsaglia-Methode werden die gleichverteilten Zahlen in gaußverteilte mit normierter Standardabweichung transformiert [KPS91]. Um die der vorgegebenen Rauschstärke $R$ entsprechende Standardabweichung zu erhalten ist eine Skalierung auf die Varianz $\sigma^{2}=\frac{2 R \Delta t}{\Delta x^{2}}$ nötig.

Gegenüber der Version des Computercodes aus [May97; May00] ist in der hier verwendeten Version der ursprüngliche Zufallszahlengenerator durch den oben zitierten ran2 ersetzt. Weiterhin wurde die Möglichkeit geschaffen, eine bereits vorhandene Höhenmorphologie einzulesen und mit dieser die weitere Entwicklung zu berechnen. Die bei der Modellierung der Bestrahlung neu auftretenden Terme sind an entsprechender Stelle in den Computercode eingefügt worden. 


\section{Experimentelle Ergebnisse}

Im Folgenden werden die Ergebnisse vorgestellt, die sich bei der Ionenbestrahlung von rauen $\mathrm{Zr}_{65} \mathrm{Al}_{7.5} \mathrm{Cu}_{27.5}$ Schichten ergeben. Die Schichten sind wie in Kap. 2.2 .2 beschrieben hergestellt. Ohne sie aus der UHV-Anlage zu entfernen, werden sie danach unter Verwendung der Ionenkanone „Ion Bombardment Gun 981-2043“ der Firma Varian mit $\mathrm{Kr}^{+}$Ionen der Energie $3 \mathrm{keV}$ bestrahlt. Der Fluss beträgt im Mittel $0.4 \mu \mathrm{A} / \mathrm{cm}^{2}$.

\subsection{Oberflächentopographien unter dem Einfluss von lonenbestrahlung}

Die Bestrahlung wird vielfach unterbrochen, um die nach einer durch Messung bekannten Fluenz erreichte Oberflächenstruktur mit dem STM zu messen. Dabei erfolgen Bestrahlung und STM-Messung, ohne dass die Probe die UHV-Kammer verlässt. Somit ist keine zwischenzeitliche Oxidation der Oberfläche zu erwarten. Es wurden für die verschiedenen Fluenzen jeweils Bilder mit den Ausschnittsgrößen $L=200 \mathrm{~nm}$ und $L=400 \mathrm{~nm}$ aufgenommen. Für beide Bildgrößen ist eine Auflösung von $400 \times 400$ Bildpunkten gewählt worden. Die typischen Parameter bei der Aufnahme der STM-Daten sind eine Tunnelspannung von $U=1.0 \mathrm{~V}$ und ein Tunnelstrom von $I=0.8 \mathrm{nA}$. Sämtliche Bilder werden einem sogenannten Plane-Fit unterzogen, bei dem eine Verkippung der Substratebene ermöglicht ist. Eine solche Transformation berücksichtigt, dass die Substratebene gegenüber der dem STM-Scanner zugrunde liegenden Ebene verkippt ist. Die daraus entstandene Bilderserie für $L=200 \mathrm{~nm}$ ist in den Abbildungen 5.1 - 5.5 zusammen mit den zugehörigen Höhen-Höhen-Korrelationsfunktionen dargestellt. Für sämtliche dargestellte Oberflächentotographien ist die Farbskala derart gewählt, dass der höchste Punkt weiß und der tiefste Punkt schwarz ist. Die zugehörige Skal ist für jedes Bild angegeben.

Die Darstellung der Oberflächentopographie aus der STM-Messung liefert bereits einen guten qualitativen Eindruck vom Effekt der Bestrahlung auf die Oberflächenstruktur (siehe Abb. 5.1 - 5.6). Auf der Oberfläche der aufgedampften und nicht bestrahlten Schicht sind Hügel einer bevorzugten Strukturgröße zu sehen. Diese Hügel liegen dicht aneinander und die Grenzen zwischen ihnen sind relativ scharf. Durch die Ionenbestrahlung werden die Grenzen zwischen den Hügeln zunehmend unscharf. Bei den niedrigen Fluenzen (bis $\Phi=4 \cdot 10^{15}$ Ionen $/ \mathrm{cm}^{2}$ ) bleibt die Strukturgröße der Hügel noch ungefähr gleich. Bei weiter steigender Fluenz heben sich die Grenzen zwischen Hügeln zum Teil auf, so dass sich einzelne Hügel zu größeren Hügeln vereinigen. Dieser Prozess setzt sich bis zu einer Fluenz von $\Phi=2 \cdot 10^{16}$ Ionen $/ \mathrm{cm}^{2}$ kontinuierlich fort. Bei weiter steigender Fluenz werden die lateral großen Hügel senkrecht zur Oberfläche (also entlang der Substratnormalen) zunehmemd kleiner, aber verschwinden noch nicht ganz. Bei Fluenzen 
von $\Phi=4 \cdot 10^{16}$ Ionen $/ \mathrm{cm}^{2}$ bis $\Phi=1 \cdot 10^{17}$ Ionen $/ \mathrm{cm}^{2}$ sind lateral kleinere Hügel auf den weiterhin vorhandenen - wenn auch schwach ausgeprägten - Hügeln mit größerer lateraler Struktur sichtbar. In diesem Fluenzbereich gibt es somit zwei verschiedene ausgezeichnete laterale Strukturgrößen. Die kleinen Hügel sind über den gesamten Bildausschnitt verteilt und haben einen Abstand zueinander, der deutlich größer als ihre Hügelgröße ist. Bei den Fluenzen $\Phi=2 \cdot 10^{17}$ Ionen $/ \mathrm{cm}^{2}$ und $\Phi=3 \cdot 10^{17}$ Ionen $/ \mathrm{cm}^{2}$ sind auch diese kleinen Hügel verschwunden. Lediglich eine sehr kleine Höhenmodulation mit großer lateraler Ausdehnung bleibt übrig. Bei der höchsten untersuchten Fluenz haben sich erneut kleine Hügel gebildet, deren Abstand untereinander deutlich größer als ihre eigene Größe ist.

Durch die Bestrahlung ist es somit insgesamt möglich, die zu Beginn vorhandenen Hügel fast vollständig zu entfernen.

Die zu den Bildern gehörigen Höhen-Höhen-Korrelationsfunktionen (zur Definition siehe Kap. 2.3.4) zeigen die im Folgenden beschriebenen charakteristischen Merkmale: Bei der unbestrahlten Oberfläche sowie bei geringen Fluenzen (bis $\Phi=4 \cdot 10^{15}$ Ionen $/ \mathrm{cm}^{2}$ ) zeigen sie im Bereich von etwa $r=20 \mathrm{~nm}$ ein Maximum oder zumindest eine ausgeprägte Schulter. Die Oberfläche bei $\Phi=1 \cdot 10^{15}$ Ionen $/ \mathrm{cm}^{2}$ bildet hier allerdings eine Ausnahme. Der Fluenzbereich bis $\Phi=4 \cdot 10^{15}$ Ionen $/ \mathrm{cm}^{2}$ entspricht dem Bereich, in dem die anfangs bestehenden Hügel noch ihrer ursprünglichen Form sehr ähnlich sind. Bei allen höheren Fluenzen ist ein Maximum im Bereich von $r=20 \mathrm{~nm}$ nicht mehr vorhanden. Es existiert aber weiterhin ein zweites Maximum. Dessen Position wandert von 30 bis $40 \mathrm{~nm}$ mit zunehmender Fluenz zu größeren Werten, bis bei $\Phi=3 \cdot 10^{17}$ Ionen $/ \mathrm{cm}^{2}$ bei der hier betrachteten Bildgröße kein zweites Maximum mehr vorhanden ist. Bei der höchsten Fluenz zeigt sich wieder ein zweites Maximum im Bereich von $45 \mathrm{~nm}$.

Bilder der Ausschnittsgröße $L=400 \mathrm{~nm}$ sollen nur für eine Auswahl von Fluenzen gezeigt werden, wenngleich sie auch für die übrigen Fluenzen ausgewertet worden sind. Als Beispiel sind Bilder der unbestrahlten, mit einer mittleren Fluenz und mit einer hohen Fluenz bestrahlten Probe in Abb. 5.6 gezeigt. Bei der unbestrahlten und der mit mittlerer Fluenz bestrahlten Probe zeigt sich, dass durch den größeren Bildausschnitt keine wesentliche zusätzliche Information gewonnen werden kann. Lediglich bei der hohen Fluenz, bei der lateral nur noch große Strukturen zurückgeblieben sind, hat der größere Bildauschnitt den Vorteil, dass nun auch hier mehrere Hügel in einem Bild liegen und somit ein zweites Maximum in der Höhen-Höhen-Korrelationsfunktion bei etwa $r=135 \mathrm{~nm}$ sichtbar ist. 

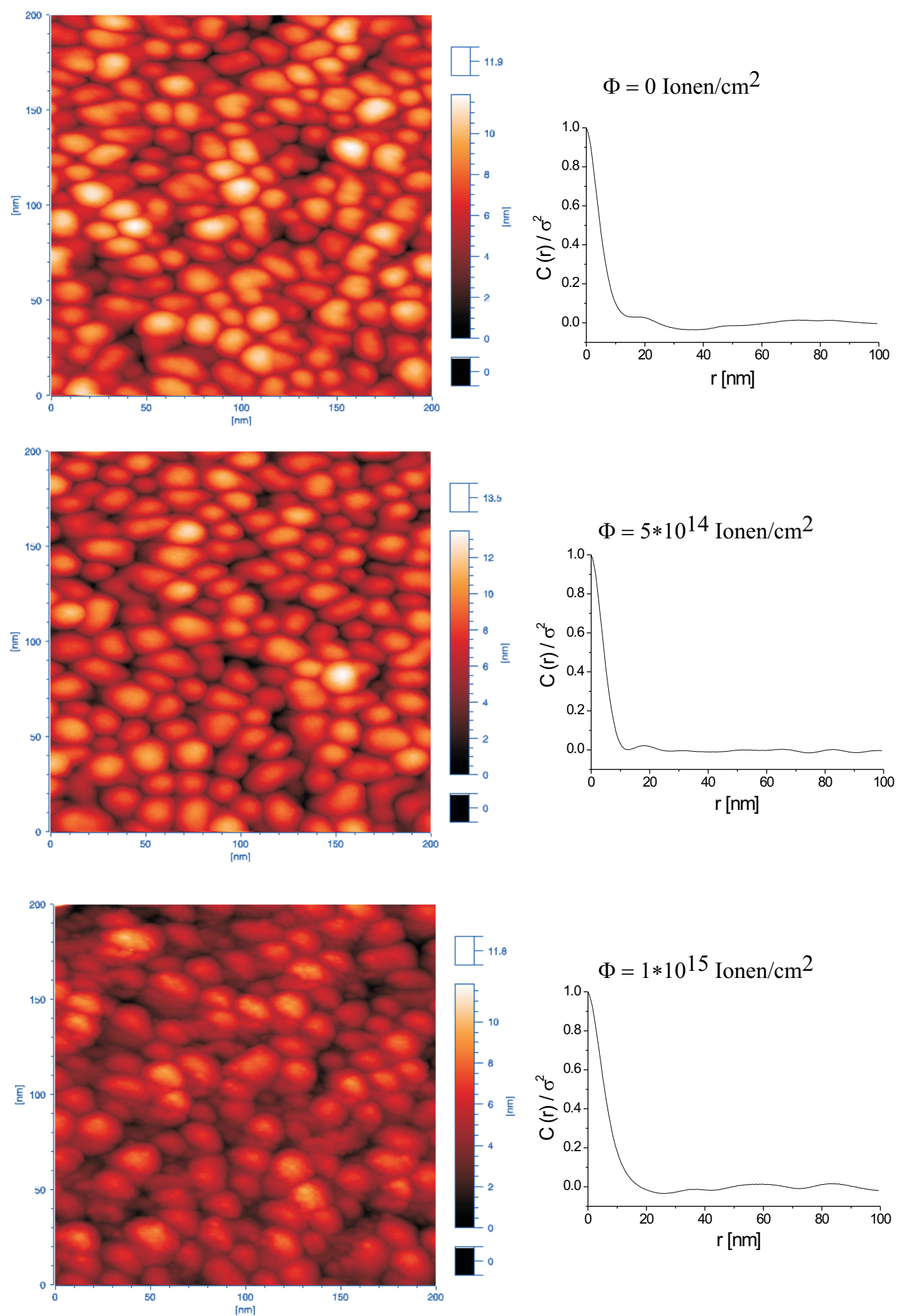

Abbildung 5.1: STM Bilder und zugehörige Höhen-Höhen-Korrelationsfunktionen für verschiedene Fluenzen (Bildgröße: $L=200 \mathrm{~nm}$, Einheit der Höhenskala: nm). 

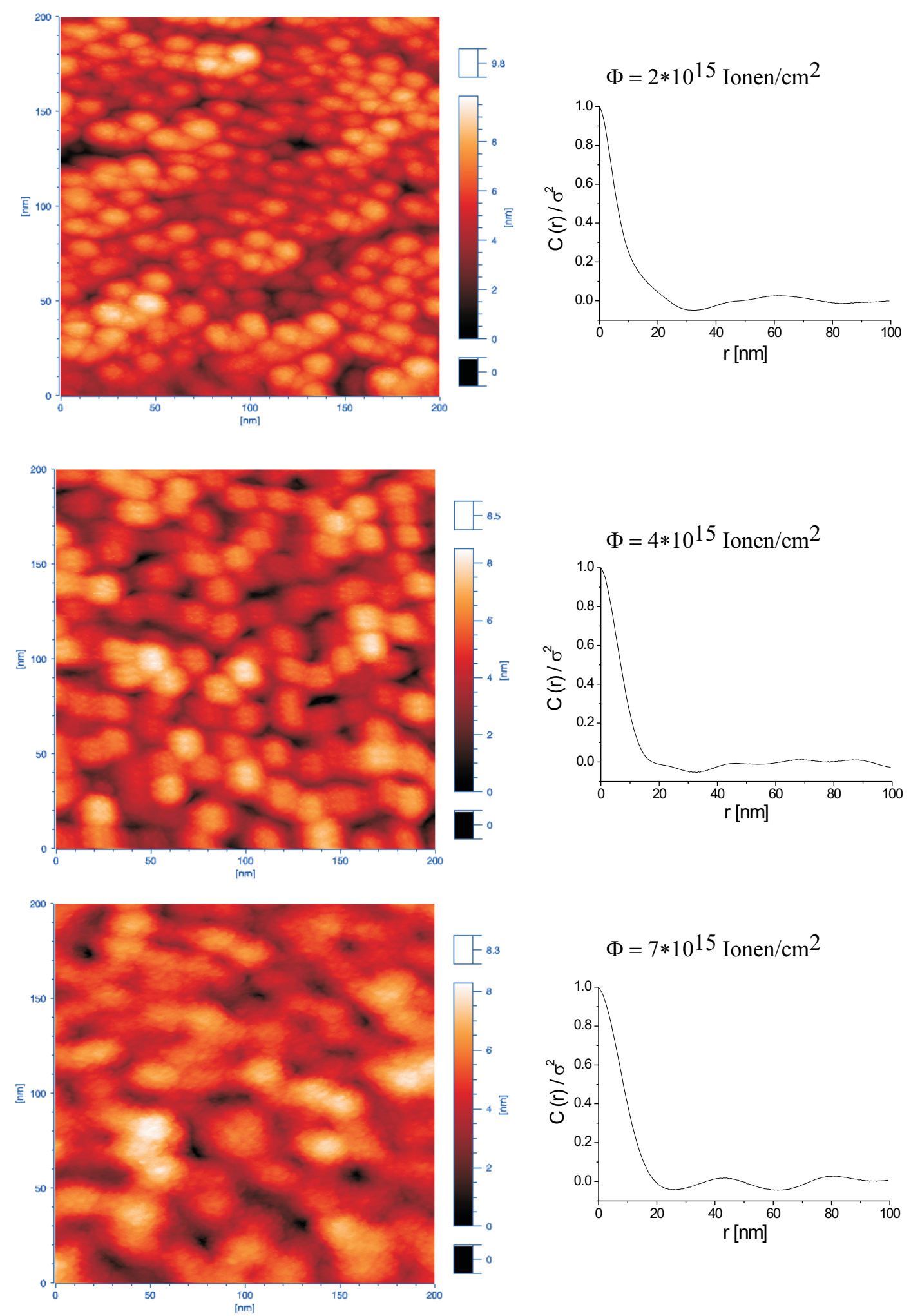

Abbildung 5.2: STM Bilder und zugehörige Höhen-Höhen-Korrelationsfunktionen für verschiedene Fluenzen (Bildgröße: $L=200 \mathrm{~nm}$, Einheit der Höhenskala: nm). 

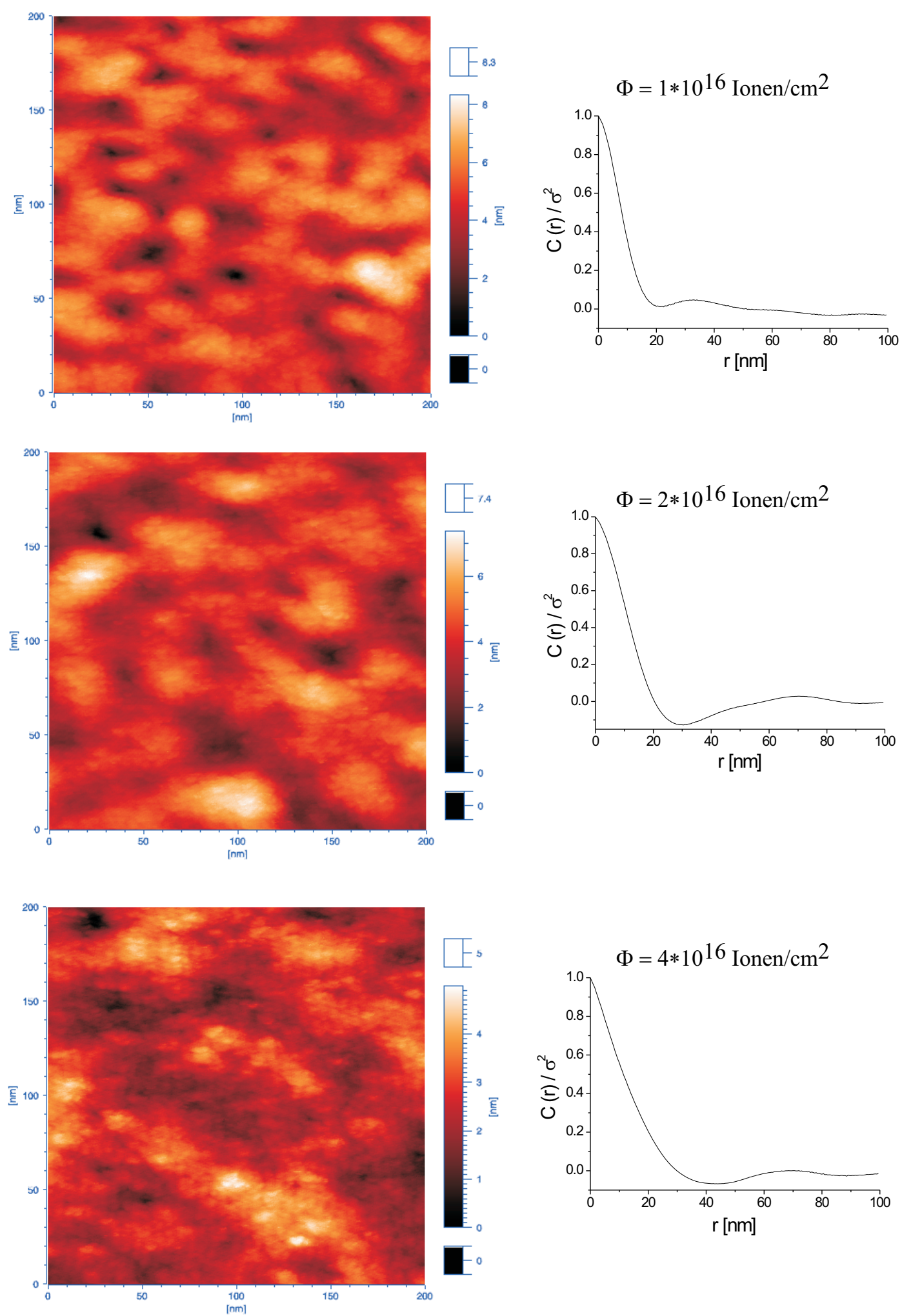

Abbildung 5.3: STM Bilder und zugehörige Höhen-Höhen-Korrelationsfunktionen für verschiedene Fluenzen (Bildgröße: $L=200 \mathrm{~nm}$, Einheit der Höhenskala: nm). 

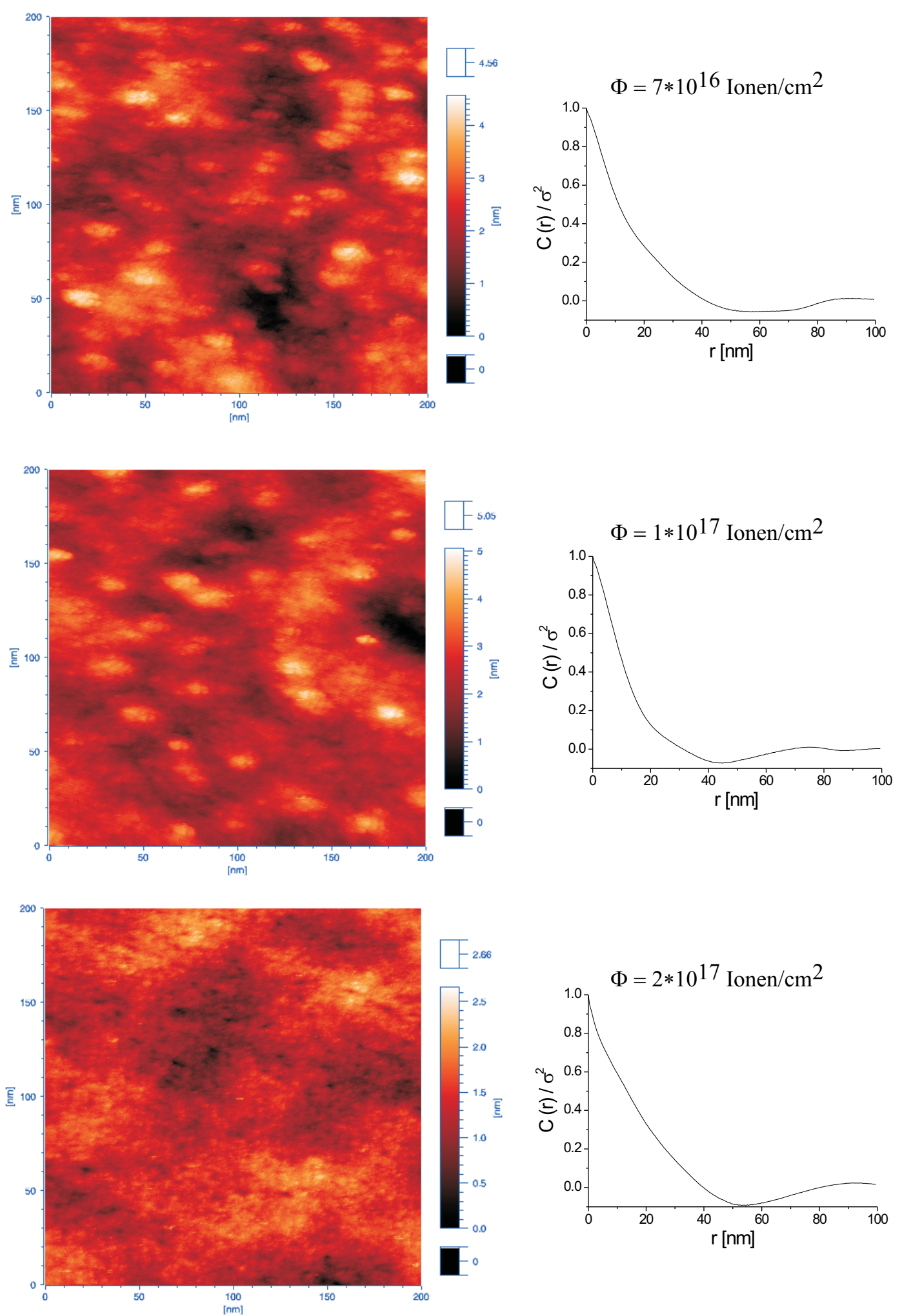

Abbildung 5.4: STM Bilder und zugehörige Höhen-Höhen-Korrelationsfunktionen für verschiedene Fluenzen (Bildgröße: $L=200 \mathrm{~nm}$, Einheit der Höhenskala: nm). 

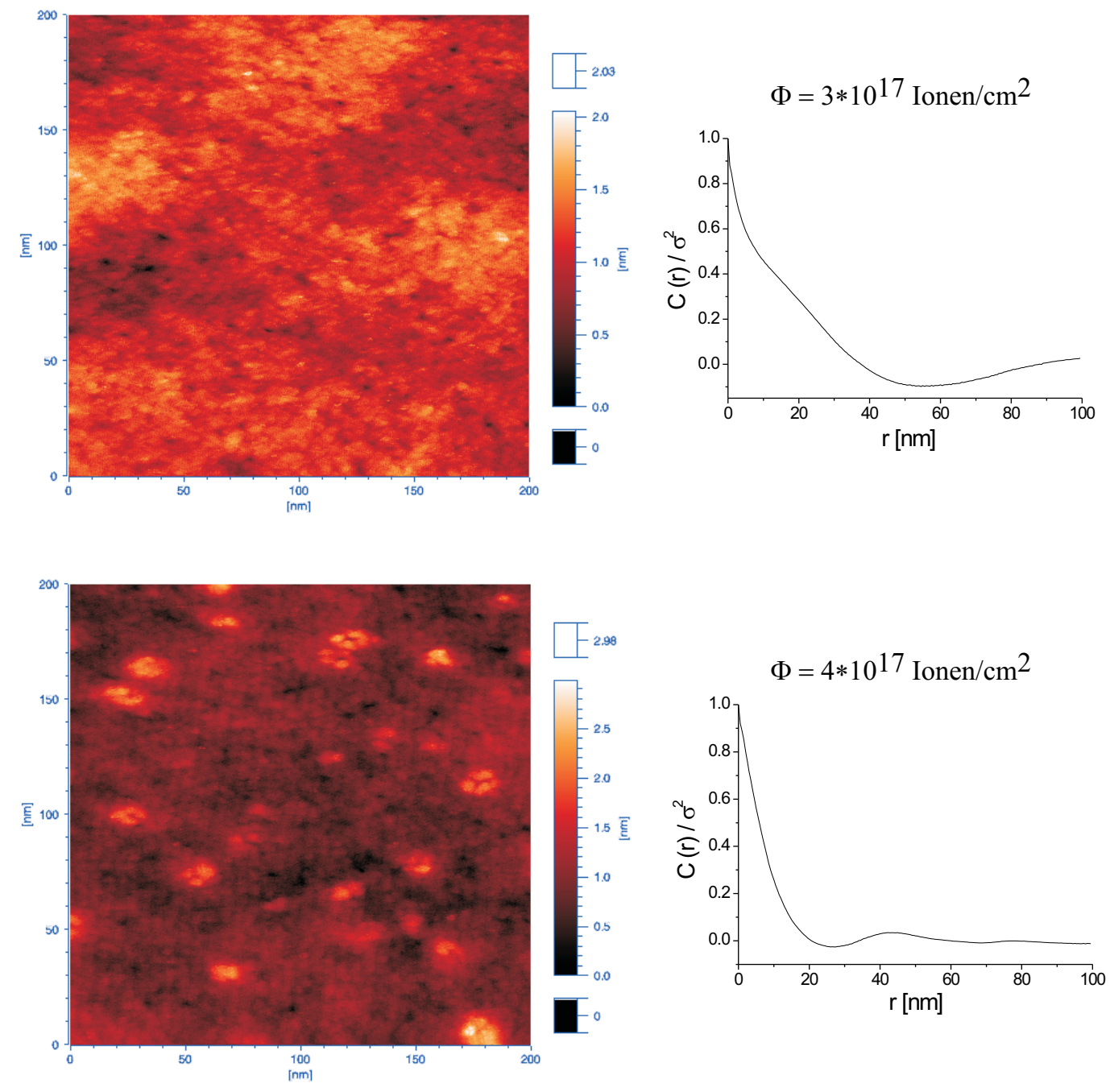

Abbildung 5.5: STM Bilder und zugehörige Höhen-Höhen-Korrelationsfunktionen für verschiedene Fluenzen (Bildgröße: $L=200 \mathrm{~nm}$, Einheit der Höhenskala: nm). 

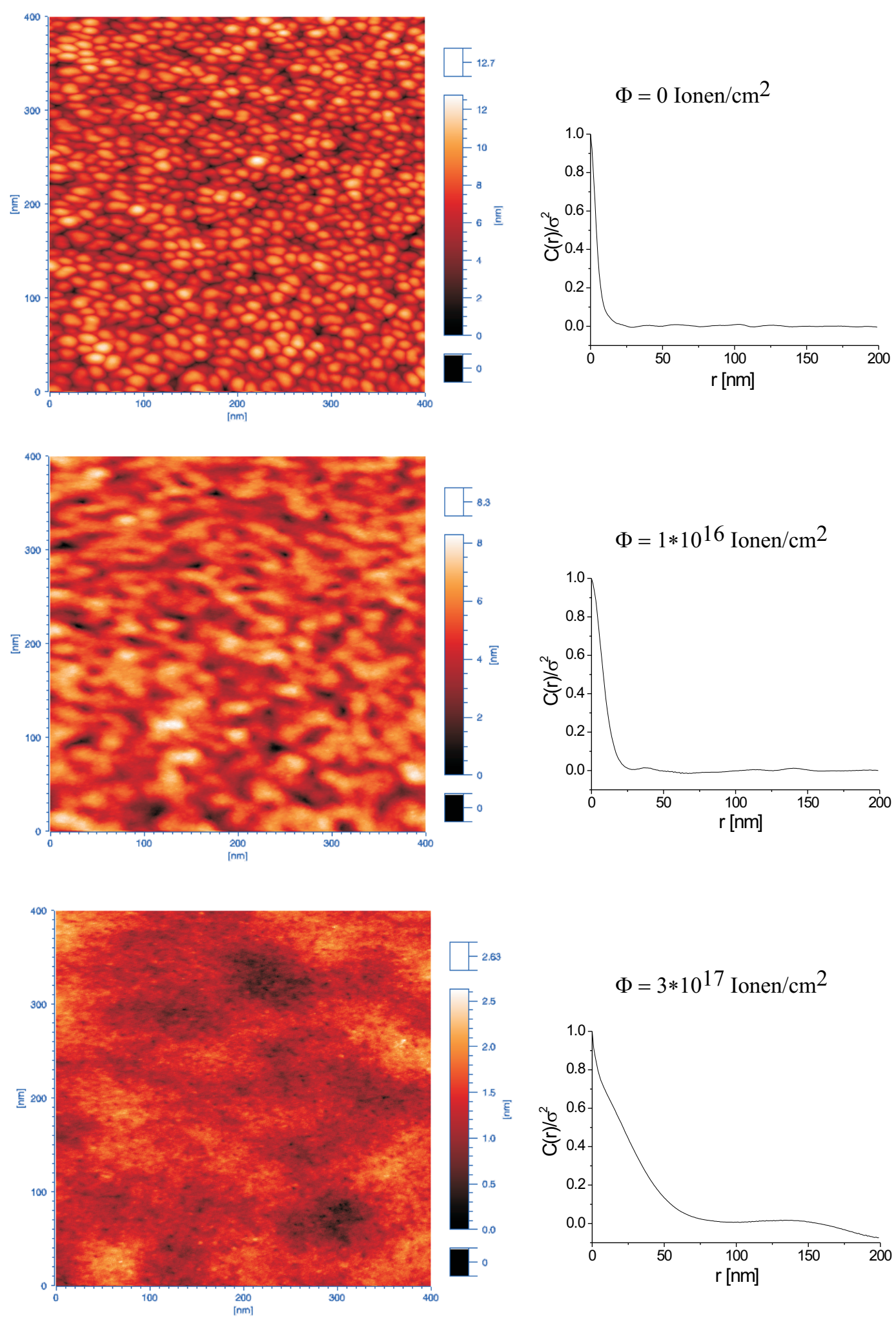

Abbildung 5.6: STM Bilder und zugehörige Höhen-Höhen-Korrelationsfunktionen für verschiedene Fluenzen (Bildgröße: $L=400 \mathrm{~nm}$, Einheit der Höhenskala: nm). 
a.)

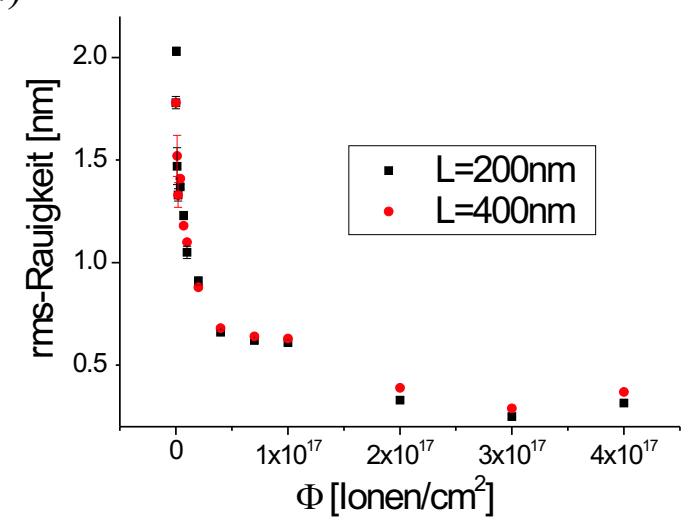

b.)

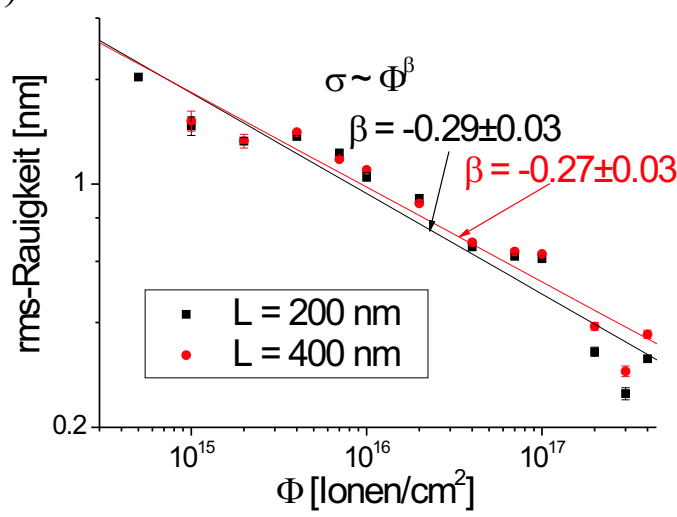

Abbildung 5.7: Rauigkeit als Funktion der Fluenz für zwei verschiedene Bildgrößen a.) in linearer Auftragung und b.) in doppeltlogarithmischer Auftragung.

\subsection{Quantitative Auswertung der Oberflächentopographien}

Für quantitative Aussagen ist für alle STM Bilder, die keinen sichtbaren Fehler enthalten, die rms-Rauigkeit berechnet worden. Damit ergibt sich für jede Fluenz ein gemittelter Wert mit zugehöriger Standardabweichung. Die entsprechenden Werte für die untersuchten Bildgrößen sind als Funktion der Fluenz in Abb. 5.7 dargestellt. Dabei sind die Werte sowohl in linearer als auch in doppeltlogarithmischer Darstellung gezeigt. In beiden Darstellungen zeigen die Ergebnisse, dass die Rauigkeit durch die Bestrahlung um etwa eine Größenordnung verringert wird. Die Rauigkeit unterscheidet sich für die einzelnen Fluenzen nur wenig für die verschiedenen Bildgrößen. Auch bei den höchsten Fluenzen, bei denen der relative Unterschied in den Rauigkeiten zwischen den Bildgrößen am größten ist, sind die Abweichungen stets kleiner als $0.06 \mathrm{~nm}$ und damit sehr klein. In der doppeltlogarithmischen Darstellung entspricht die jeweils angepasste Regressionsgerade einem Verlauf gemäß $\sigma \propto \Phi^{\beta}$. Die angepassten Geraden geben die wesentliche Tendenz der Messdaten wieder. Für den zugehörigen Exponenten ergeben sich die Werte $\beta=-0.289 \pm 0.025$ für $L=200 \mathrm{~nm}$ und $\beta=-0.272 \pm 0.024$ für $L=400 \mathrm{~nm}$. Die Werte der Exponenten zeigen somit keinen signifikanten Unterschied innerhalb des Fehlerbalkens.

Jeweils für mehrere Bilder einer Fluenz ist in den Höhen-Höhen-Korrelationsfunktionen ausgewertet worden, für welchen Abstand $R_{e}$ der Wert auf $C(0) \cdot 1 / e$ abgefallen ist (vgl. Kap. 2.3.4). Die Entwicklung der so definierten Korrelationslänge ist in Abb. 5.8.a dargestellt. Für die unbestrahlte Schicht und die mit Fluenzen bis einschließlich $\Phi=$ $1 \cdot 10^{17}$ Ionen $/ \mathrm{cm}^{2}$ bestrahlten Schichten sind die Ergebnisse für $R_{e}$ weitestgehend unabhängig von der Bildgröße. Für die höheren Fluenzen ergeben sich für die Bilder eines größeren Ausschnitts immer auch größere Korrelationslängen $R_{e}$. Unabhängig von der Bildgröße ist bis zu einer Fluenz von $\Phi=4 \cdot 10^{16}$ Ionen $/ \mathrm{cm}^{2}$ ein nahezu mono- 

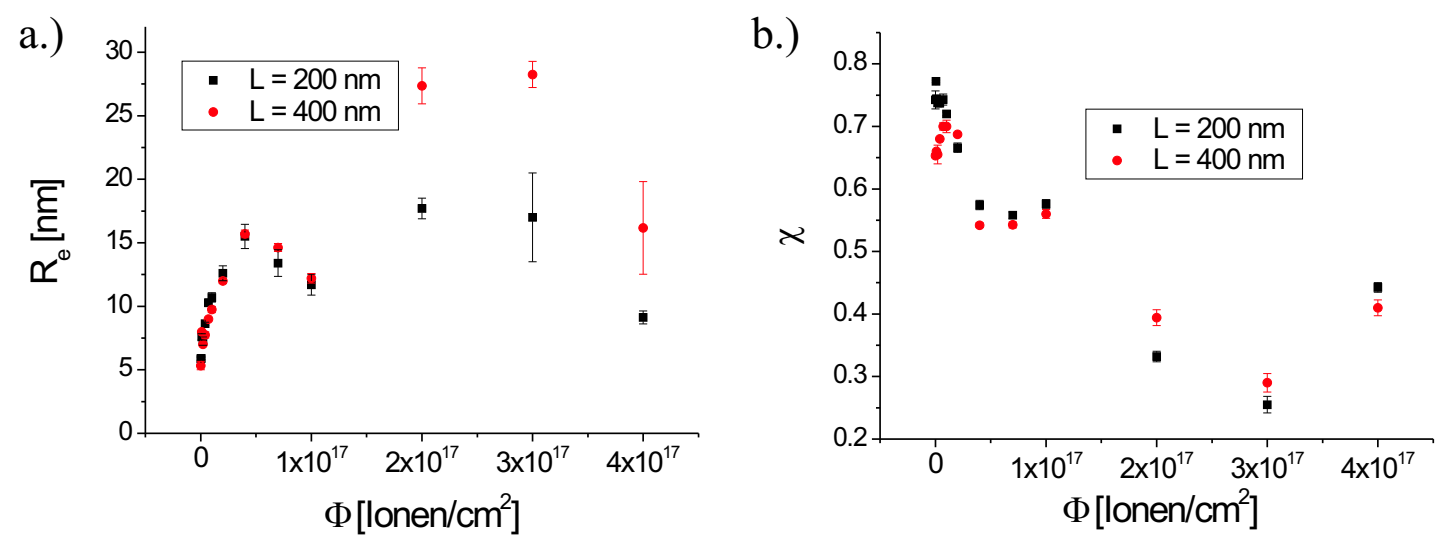

Abbildung 5.8: a.) Korrelationslängen $R_{e}$ und b.) Rauigkeitsexponenten $\chi$ als Ergebnis aus den STM-Daten für verschiedene Bildgrößen.

toner Anstieg der Korrelationslänge $R_{e}$ als Funktion der Fluenz erkennbar. Bis $\Phi=$ $1 \cdot 10^{17}$ Ionen $/ \mathrm{cm}^{2}$ schließt sich ein Rückgang an, bevor bei $\Phi=2 \cdot 10^{17}$ Ionen $/ \mathrm{cm}^{2}$ und $\Phi=3 \cdot 10^{17}$ Ionen $/ \mathrm{cm}^{2}$ die größten Werte erreicht werden. Bei der größten Fluenz ergibt sich dann wieder ein kleinerer Wert.

Ebenfalls sind die Höhen-Differenz-Korrelationsfunktionen berechnet worden (zur Definition siehe Kap. 2.3.4). Sie sind für alle dargestellten STM Bilder in Abb. 5.9 zusammen aufgetragen. Gemäß der Beziehung (4.7) lässt sich in der doppeltlogarithmischen Auftragung aus einem linearen Fit bei kleinen $r$-Werten der Rauigkeitsexponent $\chi$ bestimmen. Durch entsprechende Auswertung mehrerer STM Bilder für jede Fluenz ergeben sich die in Abb. 5.8.b dargestellten Werte. Dabei ist für die Bildgröße $L=200 \mathrm{~nm}$ die Tendenz erkennbar, dass mit steigender Fluenz die Rauigkeitsexponenten kleiner werden. Unterbrochen ist diese Entwicklung von $\Phi=4 \cdot 10^{16}$ Ionen $/ \mathrm{cm}^{2}$ bis $\Phi=1 \cdot 10^{17}$ Ionen $/ \mathrm{cm}^{2}$, wo der Rauigkeitsexponent nahezu konstant bleibt. Bei der höchsten Fluenz ist ein Anstieg des Rauigkeitsexponenten gegenüber den darunter liegenden Fluenzen deutlich. Für die Bildgröße $L=400 \mathrm{~nm}$ ergeben sich abgesehen von leichten Abweichungen bei niedrigen Fluenzen dieselben Tendenzen in der Entwicklung des Rauigkeitsexponenten.

Insgesamt zeigen die rms-Rauigkeit $\sigma$, die Korrelationslänge $R_{e}$ und der Rauigkeitsexponent $\chi$ ein korreliertes Verhalten als Funktion der Fluenz: Als grobe Tendenz geht eine Abnahme der Rauigkeit mit einer Abnahme des Rauigkeitsexponenten und einer Zunahme der Korrelationslänge einher.

Für sämtliche Fluenzen sind jeweils für mehrere STM Bilder die spektralen Leistungsdichten (zur Definition siehe Kap. 2.3.4) ausgewertet worden. Um den Einfluss der Bestrahlung auf diese Funktion darzustellen, sind sie für eine Auswahl von Fluenzen, die die verschiedenen Stadien in der Entwicklung der Oberflächentopographie repräsentieren, in Abb. 5.10 gezeigt. Mit zunehmender Fluenz verschieben sich die Daten insgesamt zu kleineren Werten. Damit wird die Fläche unter der Kurve, die wegen Gleichung (4.33) ein Maß für die Rauigkeit ist, kleiner. Insbesondere im Bereich $0.1 \mathrm{~nm}<q<0.5 \mathrm{~nm} \mathrm{nimmt}$ 


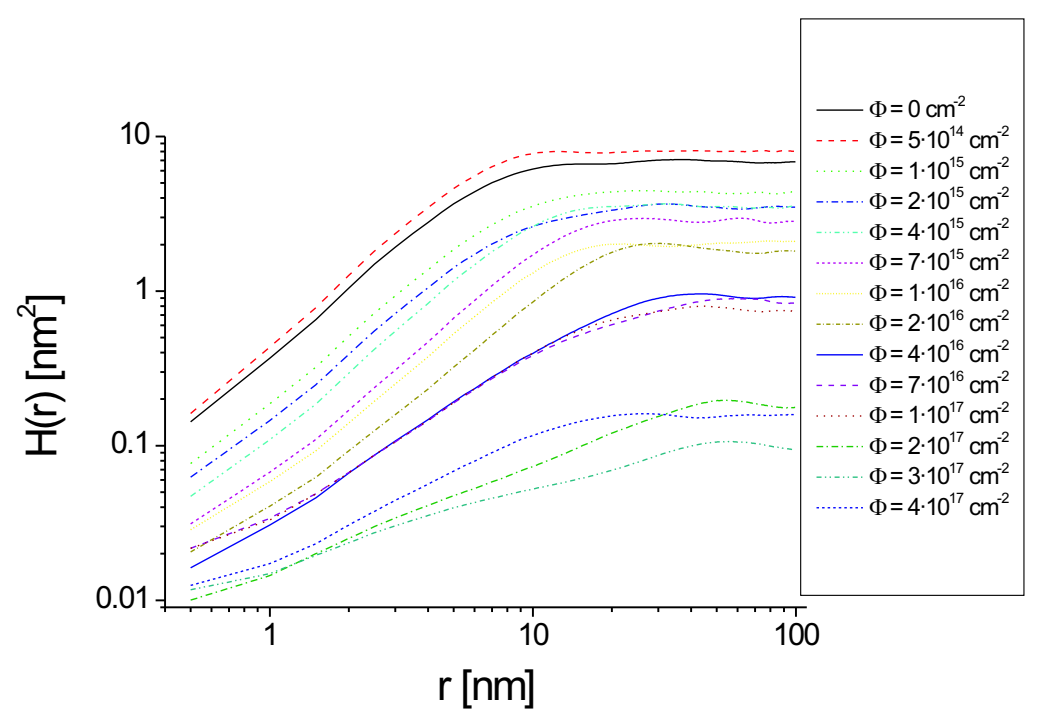

Abbildung 5.9: Höhen-Differenz-Korrelationsfunktionen der STM Bilder aus Abb. 5.15.5 .

die Intensität mit steigender Fluenz am deutlichsten ab. Diese Entwicklung stimmt mit dem Verschwinden der ursprünglich dominierenden Struktur mit Hügelgrößen von etwa $R_{c}=20 \mathrm{~nm}$ (entsprechend $q_{c}=2 \pi / R_{c}=0.314 \mathrm{~nm}^{-1}$ ) überein. Für die unbestrahlte und die mit einer Fluenz von $\Phi=3 \cdot 10^{17}$ Ionen $/ \mathrm{cm}^{2}$ bestrahlte Probe, die der glattesten Schicht entspricht, ist an den Verlauf der Kurven im Bereich großer Werte von $q$ in der doppeltlogarithmischen Darstellung eine Gerade angepasst worden. Dass diese Geraden die Werte der spektralen Leistungsdichten gut wiedergeben, bedeutet, dass sich in diesem Bereich die spektralen Leistungsdichten gemäß $C(q) \propto q^{-\zeta}$ verhalten, wobei $-\zeta$ der Steigung der Geraden entspricht. Als Werte ergeben sich für die unbestrahlte Probe $\zeta=4.34 \pm 0.04$ und $\zeta=2.96 \pm 0.03$ für die mit einer Fluenz von $\Phi=3 \cdot 10^{17}$ Ionen $/ \mathrm{cm}^{2}$ bestrahlte Probe. Die anderen Kurven zeigen, dass sich für die Steigung im Bereich großer $q$-Werte ein kontinuierlicher Übergang von dem Wert der Steigung der unbestrahlten Probe zu der mit einer Fluenz von $\Phi=3 \cdot 10^{17} \mathrm{Ionen} / \mathrm{cm}^{2}$ bestrahlten Probe ergibt. Bei allen Kurven weicht der Kurvenverlauf für die größten $q$-Werte deutlich von der Steigung in dem Bereich, in dem die Geraden angepasst werden, ab. Für die zwei hier gezeigten Kurven mit der höchsten Fluenz ergibt sich für $q>5 \mathrm{~nm}^{-1}$ ein Anstieg, während bei den anderen Fluenzen der Wert etwa konstant bleibt.

In Abb. 5.11 sind die spektralen Leistungsdichten für die unbestrahlte Probe und die mit der Fluenz von $\Phi=3 \cdot 10^{17}$ Ionen $/ \mathrm{cm}^{2}$ bestrahlte Probe erneut dargestellt. Hier sind den Modellvorstellungen entspreche Kurvenverläufe angepasst. So ist für die unbestrahlte Schicht eine Funktion der Form (4.10) angepasst worden, wobei dem Modell des Wachstums (Gleichung (4.13)) in linearisierter Form entsprechend, $b_{1}^{W}=b_{3}^{W}=0$ angenommen wird. Für die Parameter der Fitfunktion gilt $W_{j}=2 t b_{j}^{W}$ und $W_{R}=2 t R^{W}$, wobei $b_{j}^{W}$ und $R^{W}$ hier die relevanten Parameter während des Schichtwachstums sind. 


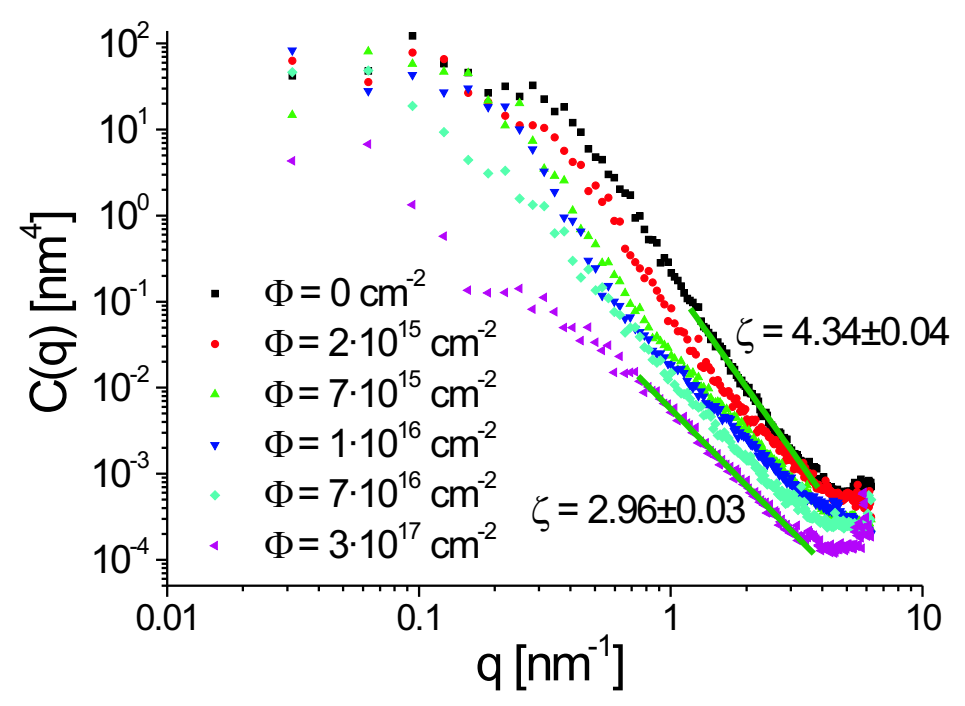

Abbildung 5.10: Spektrale Leistungsdichten für verschiedene Fluenzen.

Für die bestrahlte Probe ist die Anpassung einer Kurve der Form (4.11) notwendig. Die Funktion $C_{0}$ wird dabei durch die Parameter $W_{R}, W_{2}$ und $W_{4}$ bestimmt, die aus der Anpassung der unbestrahlten Probe bekannt und nun nicht mehr variabel sind. Als variable Parameter verbleiben die die Bestrahlungsprozesse beschreibenden $b_{j}^{F}$ und $R^{F}$, die in der Fitfunktion durch $F_{j}=2 t b_{j}^{F}$ bzw. $F_{R}=2 t R^{F}$ dargestellt sind. Den Modellvorstellungen zur Bestrahlung entsprechend wird $F_{3}=0$ gesetzt und die übrigen Parameter $F_{R}, F_{1}, F_{2}$ und $F_{4}$ sind variabel und werden angepasst. Dabei zeigt sich, dass der wesentliche Beitrag durch die Terme zweiter und vierter Ordnung geleistet wird und zwar jeweils mit $b_{2}^{F}>0$ und $b_{4}^{F}>0$. Bei beiden Anpassungen ist der Bereich der größten $q$-Werte nicht berücksichtigt worden, weil hier das Rauschen dominiert. Die genauen Werte der Fitparameter insbesondere von $F_{2}$ und $F_{4}$ sind sehr stark davon abhängig, in welchem Bereich von $q$-Werten die experimentellen Daten angepasst werden. Erstreckt sich dieser Bereich beispielsweise nur bis $q=2 \mathrm{~nm}^{-1}$, so ergibt sich für $F_{4}$ ein größerer Wert als für $F_{2}$, während die Werte für $F_{R}$ und $F_{1}$ nahe bei denen der in Abb. 5.11 gezeigten Anpassung sind.

Die spektralen Leistungsdichten sind auch für die Bilder mit einer Größe von $L=400 \mathrm{~nm}$ ausgewertet worden. Der Zerfall der spektralen Leistungsdichten ist im selben Bereich wie in Abb. 5.10 bei jeder Fluenz für mehrere Bilder ausgewertet worden. Die resultierenden Werte für $\zeta$ sind in Abb. 5.12 dargestellt. Für beide Bildgrößen ist zu beobachten, dass der Wert von $\zeta$ zunächst mit steigender Fluenz abnimmt, um zwischen $\Phi=4 \cdot 10^{16}$ Ionen $/ \mathrm{cm}^{2}$ und $\Phi=1 \cdot 10^{17}$ Ionen $/ \mathrm{cm}^{2}$ einen leicht erhöhten Wert anzunehmen und dann bei den glattesten Schichten wieder abzufallen. Zur Abhängigkeit der Werte von der Bildausschnittsgröße ist zu bemerken, dass bei der unbestrahlten Probe sowie den niedrigsten Fluenzen (bis $\Phi=7 \cdot 10^{15}$ Ionen $/ \mathrm{cm}^{2}$ ) für die größeren Bilder ein größerer Wert für $\zeta$ erreicht wird. Bei höheren Fluenzen ist kein deutlicher Einfluss der Bildgröße 
a.)

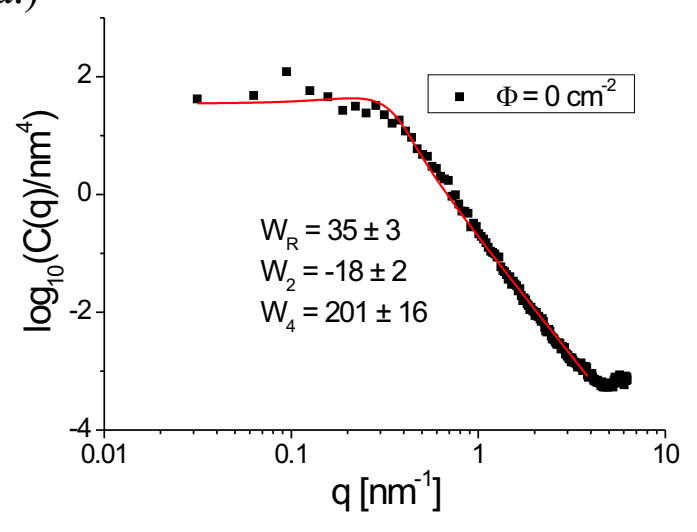

b.)

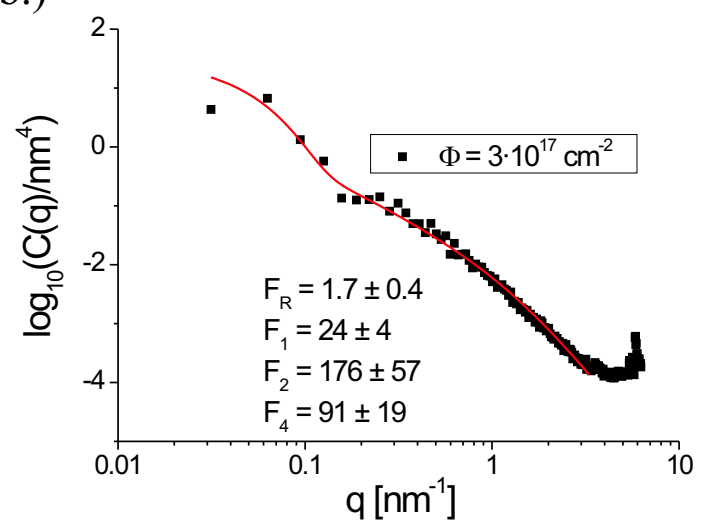

Abbildung 5.11: Spektrale Leistungsdichten für a.) die gewachsene Schicht und b.) die glatteste Schicht.

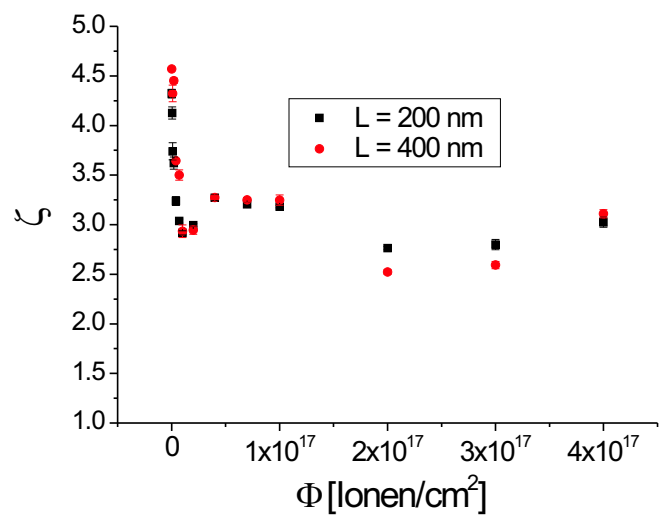

Abbildung 5.12: Exponent des Zerfalls der spektralen Leistungsdichten als Funktion der Fluenz für verschiedene Bildgrößen.

auf den Wert des Exponenten zu erkennen. Lediglich bei den glattesten Schichten ergibt sich ein etwas größerer Exponent für die Bilder der kleineren Bildauschnittsgröße.

\subsection{Analyse der Schichtdicke und Zusammensetzung}

Die gewachsene und noch nicht bestrahlte Schicht hat eine nominelle Schichtdicke von $d=480 \mathrm{~nm}$, die sich aus der Aufdampfzeit, den an den Schwingquarzwaagen gemessenen Raten und den bekannten Eichfaktoren ergibt (siehe Kap. 2.2.2). Nachdem die $\mathrm{Zr}_{65} \mathrm{Al}_{7.5} \mathrm{Cu}_{27.5}$ Schicht mit einer gesamten Fluenz von $\Phi=4 \cdot 10^{17}$ Ionen $/ \mathrm{cm}^{2}$ bestrahlt worden ist, wird ihre Schichtdicke bestimmt. Die Bestimmung der Schichtdicke mit Hilfe von Kleinwinkelröntgenreflektometrie (siehe Kap. 2.3.2.1) erwies sich an dieser Stelle als ungeeignet, weil in der Intensität innerhalb der messtechnisch bedingten Aufösung keine 


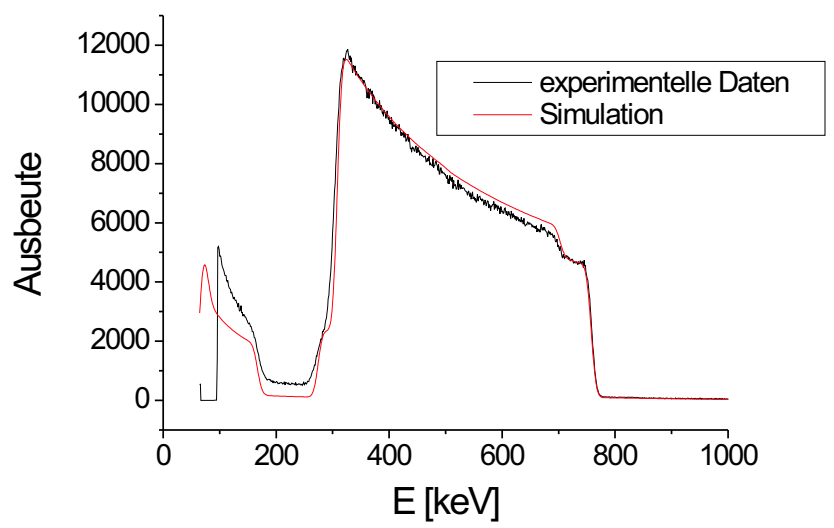

Abbildung 5.13: Signal der Rutherford-Rückstreuung (schwarz) und mit RUMP [Com04] simulierte Kurve (rot) für den Bereich in der Mitte der Probe.

Periodizität auftrat, da die Probe noch zu dick war. Als alternative Methode zur Bestimmung der Schichtdicke wurde daher die Rutherford-Rückstreuung (siehe Kap. 2.3.3) gewählt. An drei verschiedenen Orten auf der Probe, von denen einer in der Mitte und zwei am Rand der Probe liegen, ist ein Rutherford-Rückstreuungs-Spektrum aufgenommen worden. Das Spektrum in der Mitte der Probe, die sämtlichen STM Untersuchungen zugrunde liegt, ist in Abb. 5.13 dargestellt. Die angepasste Kurve stimmt in weiten Bereichen gut mit der gemessenen überein. Verstärkte Abweichungen treten lediglich im Bereich kleiner Rückstreuenergien auf, wie es bei der Kante der niedrigsten Energie erkennbar ist. Ansonsten gibt es im Bereich von etwa $400 \mathrm{keV}$ bis etwa $580 \mathrm{keV}$ eine kleine Differenz zwischen gemessener und simulierter Kurve. Für dieses Spektrum kann eine Schichtdicke von $d=420 \mathrm{~nm}$ berechnet werden. Das entspricht einem Abtrag von $60 \mathrm{~nm}$ während der Bestrahlung. Die für die Randbeeriche der Probe aufgenommenen Spektren sind dem in Abb. 5.13 gezeigten sehr ähnlich und liefern eine etwas größere Schichtdicke von $d=440 \mathrm{~nm}$. Da die STM Untersuchungen in der Probenmitte stattfanden, ist der dort gemessene Wert hier der relevante. Alle Spektren bestätigen, dass die Zusammensetzung der Schicht der gewünschten Zusammensetzung innerhalb eines Fehlers von weniger als $3 \%$ entspricht.

\subsection{Struktur der bestrahlten Probe}

Nach Durchführung der Bestrahlung ist die Schicht einer Weitwinkelröntgenmessung, wie sie in Kap. 2.3.2.2 beschrieben ist, unterzogen worden. Das Resultat dieser Messung ist in Abb. 5.14 dargestellt. Darin sind zwei über etwa zehn Grad verbreiterte Maxima zu erkennen, die beide als durch die amorphe Struktur des untersuchten Systems bedingte

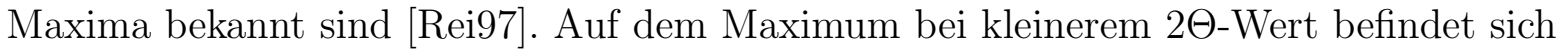
zusätzlich noch ein Maximum, das etwas schmaler ist. Die Intensität dieses zusätzlichen Maximums ist kaum größer als die der breiten Maxima. Die Flächen unter den Peaks, 


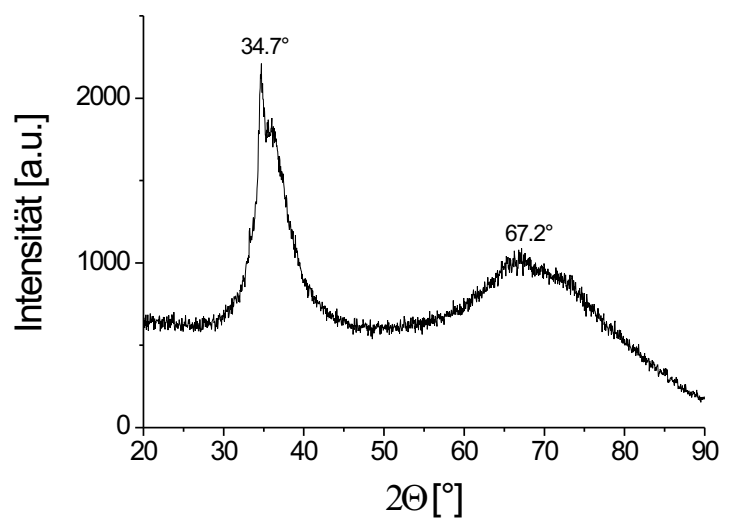

Abbildung 5.14: Weitwinkel-Röntgenmessung der $\mathrm{Zr}_{65} \mathrm{Al}_{7.5} \mathrm{Cu}_{27.5}$ Schicht nach der Bestrahlung mit einer Fluenz von $\Phi=4 \cdot 10^{17}$ Ionen $/ \mathrm{cm}^{2}$.

die dem Anteil der jeweiligen Struktur an der gesamten bestrahlten Probe proportional sind, sind für die beiden breiten Peaks deutlich größer als für den schmalen Peak.

\subsection{Diskussion}

\subsubsection{Entwicklung der Oberflächenstruktur}

Die experimentellen Untersuchungen zeigen als Hauptresultat einen deutlichen Glättungseffekt als Folge der Bestrahlung. Dieses Ergebnis stimmt in qualitativer Weise mit dem bei deutlich höheren Bestrahlungsenergien im niedrigen MeV Bereich für dasselbe Material ebenfalls beobachteten Glättungseffekt in [MA01] überein. Ebenfalls übereinstimmend bei den verschiedenen Energiebereichen ist die wesentliche Strukturentwicklung: Die klar definierten Hügel der gewachsenen Ausgangsstruktur werden zunächst unschärfer, um sich dann zu wenigeren und größeren Hügeln zu entwickeln. Quantitativ geht das mit einem Anstieg der Korrelationslänge einher.

Während im MeV Bereich die spektralen Leistungsdichten im Bereich hoher $q$-Werte einen Zerfall gemäß $C(q) \propto q^{-1}$ zeigen [MA01], der auf Volumenfließen zurückzuführen ist, wird ein solches Verhalten bei der in dieser Arbeit verwendeten Bestrahlungsenergie von $3 \mathrm{keV}$ nicht gefunden. Somit zeigen die experimentellen Ergebnisse, dass im Energiebereich einiger keV das Volumenfließen zumindest nicht der dominierende Glättungsmechanismus sein kann. Weil die spektralen Leistungsdichten hier im stationären Grenzfall der glattesten Schichten gemäß $C(q) \propto q^{-\zeta}$ mit $\zeta=2.95 \pm 0.03$ zerfallen, ist ebenfalls klar, dass nicht Oberflächendiffusion und Oberflächenfließen allein für die Strukturentwicklung verantwortlich sein können, da diese beiden Prozesse einen Wert $\zeta=4$ hervorrufen sollten. Die genauere Analyse der spektralen Leistungsdichten für die unbestrahlte Schicht und die glatteste Schicht liefern die Möglichkeit, weitere Rückschlüsse auf die beteiligten Prozesse zu ziehen (siehe Abb. 5.11). Für die unbestrahlte Probe 
ergibt sich $b_{2}^{W}<0$ und $b_{4}^{W}>0$. Das entspricht einem aufrauenden Term zweiter Ordnung und einem glättenden Term vierter Ordnung, wie es für den Wachstumsprozess bekannt ist $\left[\mathrm{RML}^{+} 00\right]$. Für die Anpassung des Leistungspektrums der glattesten Oberfläche $\left(\Phi=3 \cdot 10^{17}\right.$ Ionen $\left./ \mathrm{cm}^{2}\right)$ sind neben einem Rauschen Terme erster, zweiter und vierter Ordnung für die Anpassung zugelassen. Dabei ergibt sich für alle Ordnungen $\mathrm{j}$ unter Berücksichtigung des Fehlerbalkens stets $b_{j}^{F}=F_{j} /(2 t)>0$ als signifikantes Ergebnis (siehe Abb. 5.11). Das bedeutet, dass alle Terme glättenden Einfluss auf die Strukturentwicklung haben. Für die Ordnungen 1 und 4 sind in Kap. 4.5 ohnehin nur glättende Prozesse vorgeschlagen worden, so dass das Ergebnis mit den Erwartungen aus den Modellen übereinstimmt. Für den Term zweiter Ordnung sind in Kap. 4.5 dagegen sowohl ein glättender als auch ein aufrauender Prozess vorgestellt worden. Die Anpassung der spektralen Leistungsdichte der bestrahlten Schicht zeigt mit der Eigenschaft $b_{2}^{F}>0$, dass das ballistische Glätten im Experiment stärkeren Einfluss als die Aufrauung durch Sputtererosion hat. Weil die Parameter des Terms zweiter und vierter Ordnung sehr stark von dem Bereich, in dem die Anpassung durchgeführt wird, abhängig sind, können aus diesen Ergebnissen keine Rückschlüsse auf das quantitative Verhältnis beider Terme geschlossen werden. Eindeutig sind aber ihre Vorzeichen und die Identifikation beider Terme als glättend, sowie die Tatsache, dass sie beide einen deutlich größeren Beitrag als der Glättungsterm erster Ordnung leisten.

Dass sich für hohe Fluenzen bei den größten $q$-Werten eine Zunahme von $C(q)$ ergibt, lässt sich eventuell dadurch erklären, dass das Rauschen nicht wie in den bisherigen Modellen angenommen unkorreliert ist. Das würde bedeuten, dass die Rauschstärke $R$ von $q$ abhängig ist. Diese Beobachtung konnte auch in [MA01] gemacht werden und durch einen zusätzlichen Rauschterm auf größerer Skala erklärt werden. Im Unterschied zu den Untersuchungen bei Bestrahlungsenergien im MeV Bereich sind hier die Ausdehnungen der geschmolzenen Bereiche deutlich kleiner, so dass sich ein eventueller zusätzlicher Rauschterm bei großen $q$-Werten bemerkbar machen würde. Hier ist der Anstieg aber erst bei $q$-Werten, die im Bereich der Aufösungsgrenze des Rastertunnelmikroskops liegen, erreicht und somit nicht aussagekräftig.

Der Verlauf der Rauigkeit als Funktion der Fluenz folgt einem Potenzgesetz, wie es Abb. 5.7 zeigt. Insbesondere ist überprüft worden, dass andere mögliche Formen des Abfalls, wie exponentielles oder logarithmisches Verhalten die Daten nicht mit ähnlicher Genauigkeit wiedergeben können. Im Zusamenhang mit den Überlegungen in Kap. 4.7 zeigt das bereits, dass im linearen Modell ein Glättungsterm der Form $\frac{\partial h}{\partial t} \propto h$ keinen wesentlichen Beitrag haben sollte, sondern Glättungsterme der Ordnung $q^{j}$ mit $j \geq 1$ relevant sind.

Der Exponent $\beta$, der den Zerfall der Rauigkeit als Funktion der Fluenz beschreibt, liegt zwischen $-1 / 4$ und $-1 / 3$. In Abb. 5.7 ist die Fluenz auf der Abszisse aufgetragen, während bei der Definition des Exponenten $\beta$ in Gleichung (4.6) die Rauigkeit als Funktion der Zeit betrachtet wird. In den durchgeführten Experimenten ist die Fluenz wegen eines konstanten Ionenflusses der Zeit proportional, so dass sich für die Bestimmung des Exponenten kein Unterschied ergibt, wenn die Zeit oder die Fluenz aufgetragen wird. Im Zusammenhang mit den Ergebnissen aus Kap. 4.7 spricht der Wert des Exponenten $\beta$ dafür, dass an den Glättungsprozessen im linearen Modell zumindest einer der Ordnung 
$q^{4}$ und zumindest einer der Ordnung $q^{j}$ mit $j \leq 3$ beteiligt ist.

Die Abnahme des Rauigkeitsexponenten mit zunehmender Glättung entspricht der Erwartung, wie im Folgenden erläutert wird. Da der Rauigkeitsexponent beschreibt, wie sehr die Rauigkeit durch Vergrößerung der Bildauschnittsgröße zunimmt, bedeutet ein abnehmender Rauigkeitsexponent, dass auf Skalen unterhalb der Korrelationslänge weniger Struktur in verschiedenen Größenordnungen vorhanden ist. Die Rauigkeit nimmt also weniger zu, wenn der Bildauschnitt vergrößert wird. Dass die durch das Wachstum entwickelten Strukturen auf mittleren Skalen - also im Bereich zwischen der Größe der atomaren Aufrauung durch Bestrahlung und der während der Bestrahlung zurückbleibenden Hügel - geglättet werden, entspricht der Erwartung. Eine Abnahme des Rauigkeitsexponenten durch Ionenbestrahlung - allerdings unter anderen Bedingungen - ist im Zusammenhäng mit Glättungsprozessen bereits in früheren Experimenten beobachtet worden $\left[\mathrm{CFM}^{+} 96\right]$. Als charakteristischer Wert kann für die hier untersuchten Schichten der Wert angesehen werden, der für die glattesten Schichten bestimmt wird, weil für diese bezüglich des Glättungsprozesses der Grenzfall einer glatten Schicht erreicht ist. Damit gilt $\chi=0.29 \pm 0.02$ für die Bildauschnittsgröße $L=400 \mathrm{~nm}$, die bei den glatten Oberflächen mit großen Strukturen die physikalisch aussagekräftigeren Ergebnisse liefert, als charakteristischer Wert.

Nach Kenntnis des Rauigkeitsexponenten $\chi$ und des Exponenten $\beta$, der die für einen Glättungsprozess entsprechende Rolle des Wachstumsexponenten beim Wachstumsprozess übernimmt, stellt sich nun die Frage, ob eine Gleichung der Form (4.2) bekannt ist, die dieselben Exponenten liefert. In der Literatur sind bisher vorwiegend Wachstumsprozesse untersucht worden, die mit einer Aufrauung verbunden sind und dementsprechend Wachstumsexponenten $\beta>0$ liefern. Eine Übersicht der Exponenten zu einer Vielzahl von Gleichungen der Form (4.2) wird in [BS95] gegeben. Dort ist jedoch keine Gleichung diskutiert, die Exponenten mit den hier gefundenen Werten liefert.

Neben der Glättung verbleibt noch zu klären, warum es bei einigen Fluenzen eine Aufrauung gibt. Die mit der kleinsten Fluenz bestrahlte Probe zeigt eine etwas größere Rauigkeit als die unbestrahlte Probe. Der Aufrauungseffekt ist allerdings nicht sehr groß $\left(\sigma\left(\Phi=0\right.\right.$ Ionen $\left./ \mathrm{cm}^{2}\right)=1.78 \pm 0.03 \mathrm{~nm}, \sigma\left(\Phi=5 \cdot 10^{14}\right.$ Ionen $\left.\left./ \mathrm{cm}^{2}\right)=2.03 \pm 0.02 \mathrm{~nm}\right)$, so dass an dieser Stelle nicht eindeutig geklärt ist, ob es sich hier um einen signifikanten Effekt handelt. Eine vorübergehende Aufrauung vor Eintreten eines Glättungseffekts durch Änderung der die Oberflächenprozesse bestimmenden Bedingungen (hier: vom Wachstum zur Bestrahlung) erinnert an Ergebnisse aus Simulationen von Kontinuumsmodellen [Str04; SSM06]. Dort wird durch Modifikation des Beitrags der krümmungsinduzierten Oberflächendiffusion ein kurzfristiger Anstieg der Rauigkeit und eine Abnahme der Rauigkeit für längere Zeiten beobachtet und als Einschwingvorgang bzw. Überschwingen beim Wechsel der Koeffizienten der Wachstumsgleichung interpretiert. Ob ein solcher Effekt auch hier relevant sein kann, wird an späterer Stelle im Zusammenhang mit den Ergebnissen der Simulationen von Kontinuumsmodellen diskutiert.

Auffällig bei der Entwicklung der Oberflächenstrukturen ist, dass sich bei der höchsten untersuchten Fluenz eine neue Struktur entwickelt und die Rauigkeit zunimmt, nachdem zuvor die Oberfläche bis auf eine langwellige Modulation sehr glatt war. Dieser Effekt lässt sich dadurch erklären, dass die glättenden Mechanismen um so mehr an 
Effektivität verlieren müssen, je glatter die Oberfläche bereits ist. Unabhängig von der Oberflächenmorphologie trägt der durch die Bestrahlung bedingte Rauschprozess zur Strukturentwicklung bei. Bei den strukturierten, rauen Oberflächen ist der Beitrag des Rauschens vergleichsweise klein, so dass die Glättung dominieren kann. Je glatter die Oberfläche ist, umso mehr wird die Auswirkung des Rauschprozesses sichtbar. Dass für lange Bestrahlungszeiten bzw. für hohe Fluenzen der Rauschprozess an Einfluss gewinnt, lässt sich mit Gleichung (4.11) erklären. Die ursprüngliche Struktur geht mit der Zeit verloren und man nähert sich dem stationären Grenzfall an. Das bedeutet, dass die Rauigkeit monoton abnehmen und sich einem unteren Grenzwert, der sich aus den Werten der Rauschstärke und den Koeffizienten der beschreibenden Gleichung (siehe Gl. (4.12), (4.33)) bestimmen lässt, annähern müsste. Ein erneutes Ansteigen (und damit verbunden die Existenz eines lokalen Minimums) sagt die lineare Theorie aber nicht voraus, wenn die Koeffizienten $b_{j}$ allesamt positiv sind, wie es für glättende Prozesse gilt. Das wird klar, wenn man Gleichung (4.11) nach der Zeit ableitet, denn für $\frac{d C(q, t)}{d t}$ ergibt sich in Abhängigkeit der Zeit keine Nullstelle.

Vorstellbar ist, dass nach dem Erreichen annähernd glatter Schichten die Struktur als Funktion der Fluenz Fluktuationen unterworfen ist. Wenn der Rauschprozess mit konstanter Rauschstärke der einzige aufrauende Prozess ist, führt er bei glatten Schichten nämlich zu einem deutlicheren Rauigkeitsanstieg als bei strukturierten Oberflächen. Dieses Verhalten wird in Anhang 11.3 genauer erläutert.

Die Entwicklung des typischen Hügelradius, der durch die Korrelationslänge $R_{e}$ beschrieben ist, lässt sich quantitativ weiter analysieren. Durch doppeltlogarithmische Auftragung zeigt sich, dass auch diese Korrelationslänge einem Potenzgesetz entsprechend verläuft. Für beide Bildauschnittsgrößen ergeben sich ungefähr gleiche Exponenten. Dass die Korrelationslänge im Wesentlichen als Funktion der Fluenz ansteigt, zeigt, dass es sich beim Glättungsprozess nicht um eine Rückbildung der durch das Wachstum entstandenen Strukturen handelt. Es müssen während der Bestrahlung andere Prozesse beteiligt sein, denn während des Wachstums geht eine Zunahme der Rauigkeit mit einer Zunahme und anschließender Sättigung der Korrelationslänge einher, wohingegen sich während der Bestrahlung eine Abnahme der Rauigkeit bei weiterer Zunahme der Korrelationslänge zeigt. Während des Wachstums lässt sich der Wert der Korrelationslänge im Bereich der Sättigung durch die Wachstumsinstabilität erklären, die sich aus dem Zusammenspiel von aufrauenden und glättenden Prozessen ergibt. Wenn bei anschließender Bestrahlung für alle beitragenden Terme im linearen Modell insgesamt $b_{j}>0$ gilt, wie es zuvor gezeigt worden ist, gibt es keine Wachstumsinstabilität und somit keinen Grund für eine ausgezeichnete Korrelationslänge. Deshalb ist zu erwarten, dass die durch den Wachstumsprozess entstandene Korrelationslänge durch fortlaufende Bestrahlung verschwindet. Da nach den Aussagen des linearen Modells kleine Strukturen schneller verschwinden als große (siehe Gl. 4.11), dominieren zurückbleibende große Strukturen mehr und mehr die Struktur und die Korrelationslänge wächst. Dieses Verhalten zeigen die Ergebnisse, wenn man von der Aufrauung bei der höchsten Fluenz absieht.

Da die experimentellen Untersuchungen insgesamt eine klare Dominanz glättender 
Prozesse gegenüber aufrauenden Prozessen zeigen, ist die atomistische Untersuchung verschiedener glättender Prozesse in den folgenden MD-Simulationen Gegenstand dieser Arbeit (siehe Kap. 7).

\subsubsection{Schichtdicke, Zusammensetzung und Struktur der Probe}

Die nach der Bestrahlung durchgeführte Bestimmung der Schichtdicke ermöglicht eine Abschätzung der Sputterausbeute. Die Analyse der Schichtdicke nach der Bestrahlung hat ergeben, dass bei einer Fluenz von $\Phi=4 \cdot 10^{17}$ Ionen $/ \mathrm{cm}^{2}$ ungefähr $60 \mathrm{~nm}$ abgetragen worden sind. Nimmt man die atomare Dichte im $\mathrm{Zr}_{65} \mathrm{Al}_{7.5} \mathrm{Cu}_{27.5}$ als entsprechend der Zusammensetzung gewichtetes Mittel der Dichte der einzelnen Komponenten im jeweiligen reinen Material nach [Kit93] an, so ergibt sich daraus als Sputterausbeute $Y=0.77$. Bei der Berechnung der atomaren Dichte aus dem gewichteten Mittel der Dichten der Komponenten ergibt sich ein Wert, der vom tatsächlichen Wert der Legierung abweichen kann. Zum einen zeigt amorphes $\mathrm{Zr}_{65} \mathrm{Al}_{7.5} \mathrm{Cu}_{27.5}$ eine kleinere Dichte als kristallines [Wei98]; dies ist in Übereinstimmung mit dem Verhalten des CuTi Systems in den durchgeführten MD-Simulationen (siehe Kap. 6.1.1.1). Andererseits könnte man aufgrund günstigerer Packung für die kristalline Struktur der Legierung eher eine höhere Dichte als die dem gewichteten Mittel der Komponenten entsprechende erwarten. Insgesamt liegen solche Abweichungen im Bereich von nicht mehr als einigen Prozent. Der wesentliche Beitrag zum Fehler der Sputterausbeute resultiert aus dem Fehler, mit dem die Dicke der gewachsenen Schicht behaftet ist, denn die Schichtdicke ist groß gegenüber der Differenz dieser Dicke zur nach der Bestrahlung bestimmten. Der Wert $Y=0.77$ ist für die bestehenden Bedingungen (senkrechter Beschuss, $\mathrm{Kr}^{+}$Ionen, $\mathrm{E}=3 \mathrm{keV}$ ) im Rahmen der Erwartung, jedoch sind für die reinen Materialien in polykristalliner Form im Durchschnitt höhere Werte bekannt: Für Cu liegt der Wert etwa bei $Y=5$ und für Al bei etwa $Y=1.5$ bei einer Bestrahlung mit $\mathrm{Kr}^{+}$der Energie 3keV [AB81]. Für Zr ist ein Wert zu erwarten, der kleiner als der der anderen beiden Materialien ist, wie es Messungen mit $\mathrm{Ar}^{+}$Ionen der Energie $1.05 \mathrm{keV}$ zeigen [Oec73]. Da Zr den Hauptbestandteil des hier verwendeten Materials bildet, ist somit eine Sputterausbeute der Größenordnung $Y=1$ zu erwarten. Abweichungen zwischen dem gewichteten Mittelwert der Sputterausbeuten der polykristallinen Einzelkomponenten und der amorphen Legierung lassen sich mit den unterschiedlichen Bindungsverhältnissen erklären. Simulationen mit SRIM [ZB03] liefern dagegen einen etwas höheren Wert für die Sputterausbeute $(Y \approx 2.6)$, wenn die Standardwerte für Bindungsenergien von Oberflächenatomen verwendet werden. Da die Sputterausbeute sehr sensitiv auf Abweichungen dieser Bindungsenergien ist, sind große Abweichungen der Ergebnisse für die Sputterausbeute vom tatsächlichen Wert möglich. Abweichungen der Bindungsenergien sind insbesondere zu erwarten, weil sich die Bindungsstruktur in der amorphen Legierung von den reinen Komponenten unterscheidet.

Durch die Bestimmung der Sputterausbeute ist die Umrechnung zwischen der Fluenz und der abgetragenen Schichtdicke möglich. Diese Umrechnung ist hilfreich, weil oftmals die Dicke der abgetragenen Schicht die relevante Skala ist.

Die Abweichungen der RBS-Daten von der zugehörigen simulierten Kurve im Bereich kleiner Rückstreuenergien (vgl. Abb. 5.13) lassen sich zum Teil darauf zurückführen, dass 
Streuprozesse stattfinden, die im Simulationsprogramm nicht enthalten sind. Ein Beispiel ist Mehrfachstreuung, die besonders bei kleinen Rückstreuenergien auftritt, weil Stöße mit weit von der Oberfläche entfernten Atomen die Ursache sind. Ein weiterer Grund für Abweichungen in diesem Energiebereich ist die begrenzte Detektorauflösung. Die Abweichungen im Energiebereich von etwa $400 \mathrm{keV}$ bis $580 \mathrm{keV}$ könnten durch Abweichungen von der gewünschten Zusammensetzung im Bereich der Oberfläche bedingt sein.

Da die RBS-Messung die gewünschte Zusammensetzung der Legierung bestätigt, kann davon ausgegangen werden, dass die Schicht amorph aufgewachsen ist, wie es viele frühere Untersuchungen des Wachstums dieser Legierung bei Raumtemperatur gezeigt haben[RMS97; Rei97; MMS99]. Die nach der Bestrahlung durchgeführte Weitwinkelröntgenmessung kann bestätigen, dass die Probe auch nach der Bestrahlung weitestgehend amorph ist, denn es ergeben sich zwei Maxima, die sich über einen Bereich von mindestens $10^{\circ}$ erstrecken und in ihren Positionen denen früherer Ergebnisse von amorphen $\mathrm{Zr}_{65} \mathrm{Al}_{7.5} \mathrm{Cu}_{27.5}$ Schichten entsprechen [Rei97], und der zusätzliche schmale Peak bei $34.7^{\circ}$ liefert im Vergleich zu den anderen beiden Maxima einen kleinen Beitrag, wenn man die Fläche unter der Kurve betrachtet. Messungen zu durch Erhöhung der Substrattemperatur hergestelltem $\mathrm{Zr}_{65} \mathrm{Al}_{7.5} \mathrm{Cu}_{27.5}$ liefern kristalline Peaks an anderen Positionen [Rei97], so dass hier eine Kristallisation nicht begründet ist. Für $34.855^{\circ}$ ist ein Peak für $\mathrm{ZrO}_{2}$ bekannt [Teu62]. Die oben genannten Sputterausbeuten für die einzelnen Elemente haben gezeigt, dass die relative Sputterausbeute für Zr am kleinsten ist, so dass der relative Zr-Anteil im Oberflächenbereich erhöht wird. Das kann zu einer verstärkten Bildung von $\mathrm{ZrO}_{2}$ an der Oberfläche führen, wenn, wie hier geschehen, die Probe vor der Röntgenmessung lange an Luft gelagert war. 


\section{Thermisch aktivierte Prozesse in der MD-Simulation}

In diesem Kapitel sollen die Ergebnisse der Molekulardynamik-Simulationen zu thermisch aktivierten Prozessen dargestellt werden. Dabei beziehen sich sämtliche Ergebnisse auf Proben, die wie in Kap. 3.4 beschrieben hergestellt sind. Für die Untersuchungen werden Temperaturen gewählt, die zwar unterhalb der sich aus den Herstellungsbedingungen ergebenden Glastemperatur liegen, aber dennoch hoch genug sind, damit wesentliche Prozesse auf der Zeitskala der Simulation stattfinden können. Zunächst wird das metallische Glas CuTi und daran anschließend amorphes Si untersucht. Beide Systeme werden in einem späteren Kapitel bezüglich der Bestrahlung durch energetische Ionen behandelt und analysiert, so dass ein Vergleich zwischen thermisch aktivierten und strahlungsinduzierten Prozessen für beide Systeme möglich ist.

Für beide Materialklassen soll am Beginn des jeweiligen Kapitels gezeigt werden, dass in der MD-Simulation ein Glas hergestellt wird. An diese notwendige Vorarbeit schließt sich im weiteren Verlauf die Untersuchung der Dynamik in gläsernen dünnen Schichten an, die für dieses Kapitel von zentraler Bedeutung ist.

In dem nun folgenden Kapitel über die CuTi Legierungen wird in jedem Abschnitt zunächst die Analysemethode der Daten im Zusammenhang mit dem theoretisch erwarteten Verhalten erläutert, bevor anschließend die entsprechenden Ergebnisse vorgestellt werden. Im anschließenden Kapitel über Si werden dieselben Methoden verwendet, so dass dort direkt auf die Ergebnisse eingegangen werden kann.

Die wesentlichen Ergebnisse zum dynamischen Verhalten der CuTi Schichten sind in verkürzter Darstellung auch in [VM05] zu finden.

\subsection{CuTi Legierungen}

Als Modellsystem für ein metallisches Glas wird hier CuTi in den Zusammensetzungen $\mathrm{Cu}_{40} \mathrm{Ti}_{60}, \mathrm{Cu}_{50} \mathrm{Ti}_{50}$ und $\mathrm{Cu}_{60} \mathrm{Ti}_{40}$ verwendet. Neben der in Kap. 3.3.2 gelieferten Begründung für die Wahl dieses Materialsystems ist zu erwähnen, dass es auch experimentelle Untersuchungen für verschiedene Zusammensetzungen glasartiger CuTi Legierungsschichten gibt [Hü198; GHK98], so dass der Einfluss der Zusammensetzung der Legierung in Verbindung mit hier durchgeführten Simulationen interpretiert werden kann. Bei Vergleichen zwischen Experiment und Simulation ist aber stets zu beachten, dass im für $\mathrm{CuTi}$ verwendeten Wechselwirkungspotential (siehe Kap. 3.3.2.1) keine kovalenten Bindungsanteile vorhanden sind, während in der Realität solche gerichteten Bindungsanteile nicht auszuschließen sind. Deshalb ist das simulierte Glas im Vergleich zu den 
realen Gläsern in seinem Verhalten sicherlich als fragiler einzuschätzen.

\subsubsection{Herstellung, Struktur und Glasübergang}

Die Probenherstellung in der MD-Simulation erfolgt wie in Kap. 3.4 beschrieben. Zunächst soll nun dargelegt werden, dass dabei tatsächlich glasartige Proben hergestellt werden. Als Indikatoren dafür werden verschiedene Eigenschaften von Gläsern herangezogen. Der Glasübergang spiegelt sich in thermodynamischen Größen wider, die strukturelle Unordnung in der Paarkorrelationsfunktion und einer geänderten Dichte gegenüber dem Kristall.

\subsubsection{Thermodynamische Größen und Glasübergang}

Begriffe und Erwartungen Ein Glasübergang macht sich dadurch bemerkbar, dass bei der entsprechenden Temperatur das spezifische Volumen und die Enthalpie einen Knick bzw. ihre ersten Ableitungen nach der Temperatur einen Sprung zeigen [Zar91]. Die Temperatur, bei der dieser Knick stattfindet, entspricht der Glastemperatur $T_{g}$. Wegen der Nichtgleichgewichtsprozesse stellt es einen Unterschied dar, ob das Volumen bzw. die Enthalpie als Funktion der Temperatur während des Abkühlens aus der Flüssigkeit in den Glaszustand oder während des Heizens aus dem Glaszustand in den flüssigen Zustand hinein aufgenommen wird. In einem Übersichtsartikel von Faupel et al. [FFM ${ }^{+} 03$ ] wird daher zwischen beiden Übergangstemperaturen $T_{g}^{K}$ (Kühlen) und $T_{g}^{H}$ (Heizen) unterschieden. Außerdem sind beide Übergangstemperaturen von der Kühl- bzw. der Heizrate abhängig [El190; Zar91]. Experimentell ist in der Regel $T_{g}^{H}$ leichter zugänglich, indem man die spezifische Wärme während eines Aufheizvorganges bestimmt. Man nutzt dabei aus, dass die spezifische Wärme der Ableitung der zugeführten Wärme (also bei isobaren Prozessen der Enthalpie) nach der Temperatur entspricht, und erwartet daher einen Sprung beim Glasübergang. Beim Abkühlen aus der Schmelze müssen die Kühlraten hoch sein, um den Glaszustand zu erreichen, so dass ein gleichzeitiges Messen thermodynamischer Größen schwierig ist. In der Simulation lassen sich dagegen auch bei hohen Raten Enthalpie und Volumen sowohl für das Heizen als auch für das Abkühlen einfach beobachten.

Die unterschiedliche strukturelle Anordnung einer gläsernen Probe gegenüber der kristallinen Anordnung im Gleichgewichtszustand äußert sich außerdem durch unterschiedliche Massendichten. Dabei ist in der Regel die Dichte im kristallinen Zustand etwas höher als im Glas [Ell90].

Ergebnisse Die Proben der CuTi Legierungen werden wie in Abschnitt 3.4 beschrieben hergestellt. Während des Heizens und Abkühlens bei konstantem Druck werden die momentane Größe der Simulationsbox und die Temperatur ausgegeben. Daher ergibt sich die Möglichkeit, das Volumen in Abhängigkeit der Temperatur darzustellen, wie es in Abb. 6.1 für die $\mathrm{Cu}_{50} \mathrm{Ti}_{50}$ Probe zu sehen ist. Beim Abkühlen aus der Schmelze ergibt sich im Bereich unterhalb von $T=1000 \mathrm{~K}$ eine Änderung der Steigung. Um 

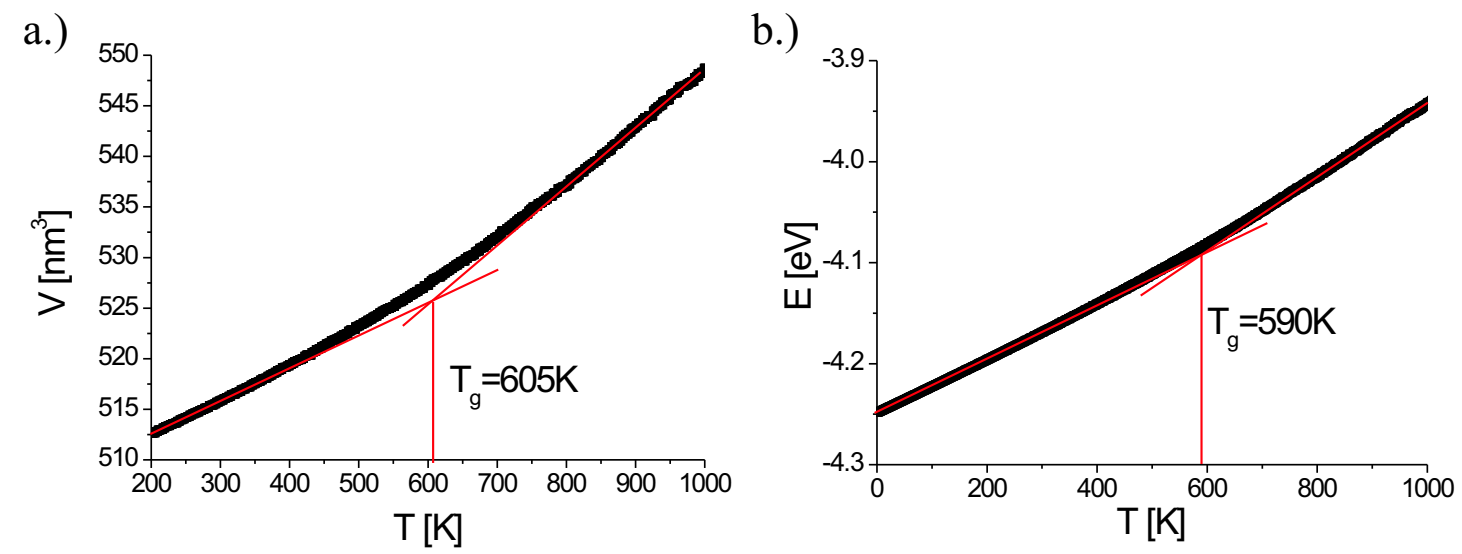

Abbildung 6.1: a.) Volumen und b.) Energie jeweils in Abhängigkeit der Temperatur für $\mathrm{Cu}_{50} \mathrm{Ti}_{50}$ im Bereichs $\mathrm{T} \leq 1000 \mathrm{~K}$ beim Abkühlen mit linearen Fits (Rate: $\dot{T}=-20 \mathrm{~K} / \mathrm{ps}$ ).

diese Änderung herum kann man die Kurve linearisieren und erhält so aus dem Schnittpunkt beider Geraden eine Temperatur als Position des Knicks. Diese Temperatur kann als Glastemperatur des Kühlprozesses $T_{g}^{K}$ interpretiert werden. Nach diesem Heiz- und Kühlzyklus wird die Probe nochmals von $T=0 \mathrm{~K}$ auf $T=2000 \mathrm{~K}$ geheizt und zwar mit derselben Temperaturrate, mit der zuvor abgekühlt wurde. Die Position des Knicks wird wieder auf die gleiche Art bestimmt und daraus ergibt sich eine Glastemperatur des Heizprozesses $T_{g}^{H}$. Man erhält ganz ähnliche Kurvenverläufe, wenn man diese Prozedur für die $\mathrm{Cu}_{40} \mathrm{Ti}_{60}$ und $\mathrm{Cu}_{60} \mathrm{Ti}_{40}$ Proben durchführt. Die Ergebnisse für die entsprechenden Glastemperaturen sind in Tab. 6.1 zusammengefasst.

Außerdem macht sich ein Glasübergang auch durch einen Knick in der Auftragung der Enthalpie als Funktion der Temperatur bemerkbar [Zar91]. Für alle Probenherstellungen der CuTi Legierungen gibt es aber keinen Unterschied zwischen der Energie und der Enthalpie, weil der Prozess jeweils bei $p=0$ mbar durchgeführt wird. Für die $\mathrm{Cu}_{50} \mathrm{Ti}_{50}$ Probe ist der entsprechende Verlauf in Abb. 6.1 zu sehen. Dort ist die Energie pro Atom als Funktion der Temperatur aufgetragen. Mit sinkender Temperatur zeigt sich während der Abkühlung ein linearer Bereich, bis unterhalb von $T=1000 \mathrm{~K}$ wieder ein Knick auftritt, der in Abb.6.1 dargestellt ist. Die diesem Knick entsprechende Temperatur kann man als aus der Enthalpie bestimmte Glastemperatur des Kühlprozesses $T_{g}^{K}$ bezeichnen. Auch während des anschließenden Heizens bei gleicher Rate wird die Enthalpie bzw. Energie als Funktion der Temperatur aufgetragen und die entsprechende Glastemperatur $T_{g}^{H}$ aus der Position des Knicks bestimmt. Die anderen Zusammensetzungen werden analog behandelt und sämtliche Ergebnisse für die Glastemperaturen sind in Tab. 6.1 dargestellt.

Damit liegen die Werte für $T_{g}^{K}$ und $T_{g}^{H}$ für alle Zusammensetzungen relativ weit auseinander. Andererseits ist für jede Zusammensetzung der Wert von $T_{g}^{K}$ und $T_{g}^{H}$ weitestgehend unabhängig davon, ob er über das Volumen oder die Enthalpie bestimmt 


\begin{tabular}{|l|l|l|l|}
\hline & $\mathrm{Cu}_{40} \mathrm{Ti}_{60}$ & $\mathrm{Cu}_{50} \mathrm{Ti}_{50}$ & $\mathrm{Cu}_{60} \mathrm{Ti}_{40}$ \\
\hline$T_{g}^{K}[\mathrm{~K}]$ aus $V(T)$ & 595 & 605 & 585 \\
\hline$T_{g}^{H}[\mathrm{~K}]$ aus $V(T)$ & 761 & 758 & 742 \\
\hline$T_{g}^{K}[\mathrm{~K}]$ aus $E(T)$ & 595 & 590 & 604 \\
\hline$T_{g}^{H}[\mathrm{~K}]$ aus $E(T)$ & 757 & 768 & 767 \\
\hline
\end{tabular}

Tabelle 6.1: Glasübergangstemperaturen der CuTi Legierungen während des Kühl- und Heizprozesses bestimmt aus $V(T)$ und $E(T)$ (Rate: $\dot{T}= \pm 20 \mathrm{~K} / \mathrm{ps}$ ).

wird. Außerdem unterscheiden sich die Übergangstemperaturen in Abhängigkeit von der Zusammensetzung nicht signifikant. Die zugehörigen Abweichungen liegen im Rahmen des durch die Bestimmungsmethode bedingten Fehlers, der als etwa $10 \mathrm{~K}$ angenommen werden kann.

Um die Erwartung bezüglich der Massendichten von glasartigen gegenüber kristallinen Proben hier zu überprüfen, wird zum einen die auf $T=0 \mathrm{~K}$ abgeschreckte amorphe $\mathrm{Cu}_{50} \mathrm{Ti}_{50}$ Probe verwendet. Zum anderen wird die $\mathrm{Cu}_{50} \mathrm{Ti}_{50}$ Ausgangsprobe in der kristallinen B11 Struktur der $\gamma$-Phase (siehe Kap. 3.4) bei derselben Temperatur für einige Zeit relaxiert, bis sich Gleichgewichtsabstände zwischen den Atomen eingestellt haben. Diese Probe dient nun als Referenzprobe. Als relative Änderung des atomaren Volumens erhält man $\frac{V_{\text {amorph }}-V_{\text {kristallin }}}{\frac{1}{2}\left(V_{\text {amorph }}+V_{\text {kristallin }}\right)}=1.54 \%$, d.h. das spezifische Volumen ist im Amorphen etwas größer als im Kristall.

Zusammenfassend kann man bis hierher sagen, dass die thermodynamischen Größen einen Glasübergang zeigen (siehe auch Diskussion in Kap. 6.2.1). Somit ist davon auszugehen, dass die Probe deutlich unterhalb der entsprechenden Temperatur für die relevanten Zeitskalen der im Folgenden durchgeführten Untersuchungen im Glaszustand ist.

\subsubsection{Atomare Struktur und Ordnung}

Begriffe und Erwartungen Der amorphe Zustand unterscheidet sich in seiner Struktur deutlich vom kristallinen. Während im Kristall eine langreichweitige Ordnung und eine periodische Struktur vorliegen, ist beides im amorphen Zustand nicht vorhanden. Im Amorphen gibt es nur eine kurzreichweitige Nahordnung. Im Realraum werden diese unterschiedlichen Strukturen in der Paarkorrelationsfunktion wiedergegeben und im reziproken Raum durch den entsprechenden Strukturfaktor [Was80]. Bei der Paarkorrelationsfunktion wird gezählt, wie viele Atome es gibt, die einen gewissen Abstand $r$ zueinander haben. Im dreidimensionalen Raum nimmt die Anzahl bei homogener Verteilung entsprechend einer Kugeloberfläche mit $4 \pi r^{2}$ zu. Um diesen Effekt herauszufiltern und nur die für die Struktur relevante Informationen zu bekommen, verwendet man wie in [AT87] unter Berücksichtung endlicher Intervallbreiten $\Delta r$ bei der Berechnung [Rap95]

$$
g(r)=\frac{V}{N^{2}} \frac{1}{4 \pi r^{2} \Delta r} \sum_{i \neq j} \delta_{\Delta r}\left(r-\left|\vec{r}_{i}-\vec{r}_{j}\right|\right) \text { mit } \delta_{\Delta r}(x)= \begin{cases}1 & \text { für }|x| \leq \Delta r / 2 \\ 0 & \text { sonst }\end{cases}
$$


Dabei ist $\mathrm{N}$ die Anzahl der Atome und $\mathrm{V}$ das von ihnen eingenommene Volumen. Diese Paarkorrelationsfunktion sollte sich dann für große Abstände dem Grenzwert $\lim _{r \rightarrow \infty} g(r)=1$ annähern. Während im Experiment eher der Strukturfaktor durch Streuexperimente zugänglich ist, nutzt man in der atomistischen Simulation die direkte Kenntnis der Atompositionen im Realraum.

Für den Kristall erwartet man aufgrund der Fernordnung viele schmale Peaks in der Paarkorrelationsfunktion, auch bei größeren Entfernungen. Sowohl in der Flüssigkeit als auch im Amorphen erwartet man verbreiterte Peaks aufgrund der Nahordnung durch die umgebenden Nachbarn, deren Nachbarn, usw.. Aufgrund der statistisch verteilten Anordnung sind die Peaks im Vergleich zur kristallinen Struktur allerdings verbreitert und von geringerer Intensität [El190]. Mit der Entfernung nimmt die Korrelation ab, so dass die Intensität der Peaks mit zunehmender Ordnung abnimmt. Der Unterschied zwischen einer amorphen und einer flüssigen Struktur wird dadurch widergespiegelt, dass die Paarkorrelationsfunktion für die Flüssigkeit eine glattere Kurve liefert, weil die thermischen Bewegungen der einzelnen Atome stärker sind. Für metallische Gläser ist außerdem aufgrund der bestehenden Nahordnung eine Aufteilung des zweiten Peaks in zwei einzelne Peaks typisch [Was80].

Zur Berechnung der Paarkorrelationsfunktionen wird ein Computercode von S. G. Mayr verwendet. Die damit erhaltenen Resultate werden Gleichung (6.1) entsprechend normiert.

Ergebnisse Beispielhaft ist in Abb. 6.2 die Paarkorrelationsfunktion der $\mathrm{Cu}_{50} \mathrm{Ti}_{50}$ Probe zu verschiedenen Zeitpunkten gezeigt: direkt nach dem Abschrecken aus der Schmelze bei $T=0 \mathrm{~K}$ und nach Abschluss der anschließend, bei $T=450 \mathrm{~K}$ durchgeführten und in Kap. 6.1.2 beschriebenen, Diffusionsuntersuchungen. Für die Zuordnung der Zeitpunkte zum Herstellungsprozess sei außerdem auf Kap. 3.4 verwiesen. In beiden Fällen entsprechen die Verläufe einer nichtkristallinen Struktur, denn die vorhandenen Peaks klingen mit dem Abstand deutlich ab und es sind keine scharfen Peaks bei größeren Abständen sichtbar. Bei der höheren Temperatur sind im Vergleich zur tieferen Temperatur alle Peaks etwas nach rechts verschoben, wie man es wegen thermischer Ausdehnung erwartet. Ansonsten ist der auffälligste Unterschied zwischen beiden Kurven, dass bei $T=0 \mathrm{~K}$ der zweite Peak deutlich in zwei Einzelpeaks zerfällt, während bei $T=450 \mathrm{~K}$ diese Teilung zwar noch sichtbar aber weniger stark ausgeprägt ist.

Die Proben der anderen Zusammensetzungen zeigen in den wesentlichen Punkten das gleiche Verhalten wie $\mathrm{Cu}_{50} \mathrm{Ti}_{50}$ (siehe Abb. 6.3). Für alle Zusammensetzungen spaltet der zweite Peak bei $T=0 \mathrm{~K}$ in zwei Maxima auf. Dieses Aufspalten ist bei $T=450 \mathrm{~K}$ je nach Zusammensetzung unterschiedlich stark zu sehen. Dabei nimmt die Deutlichkeit der Aufspaltung bei $T=450 \mathrm{~K}$ mit abnehmendem Kupferanteil ab. Trotzdem kann man bei allen Zusammensetzungen immer noch erkennen, dass der zweite Peak sich aus zwei Einzelpeaks zusammensetzt. Außerdem ändert sich im aufgespaltenen zweiten Peak die relative Höhe der Teilpeaks mit der Zusammensetzung. Je größer der Kupferanteil ist, desto höher ist der linke Teilpeak im Verhältnis zum rechten. Bei der $\mathrm{Cu}_{40} \mathrm{Ti}_{60}$ Probe ist bei $T=0 \mathrm{~K}$ im Gegensatz zu allen anderen Kurven auch eine Aufspaltung des ersten 


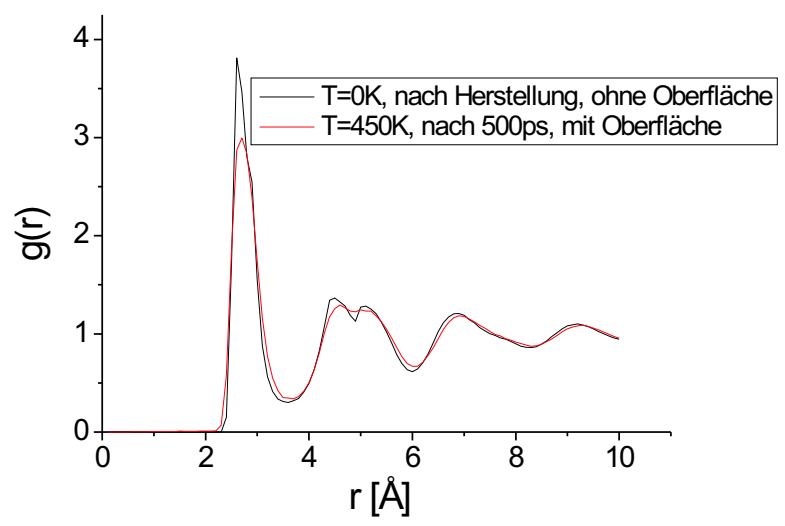

Abbildung 6.2: Paarkorrelationsfunktion von $\mathrm{Cu}_{50} \mathrm{Ti}_{50}$ direkt nach dem Abschreckprozess bei $T=0 \mathrm{~K}$ ohne Oberflächen (schwarze Linie) und nach Abschluss der Diffusionsuntersuchungen über 500 ps bei $T=450 \mathrm{~K}$ mit Oberflächen in einer Richtung (rote Linie).

Peaks zu erkennen.

Insgesamt zeigen die Paarkorrelationsfunktionen sowohl direkt nach der Herstellung ohne Oberflächen bei $T=0 \mathrm{~K}$ als auch nach Abschluss der Diffusionsuntersuchungen bei $T=450 \mathrm{~K}$ mit Oberflächen die typischen Merkmale eines metallischen Glases (siehe auch zugehörige Diskussion in Kap. 6.2). Deshalb wird im Folgenden davon ausgegangen, dass alle Proben über den gesamten Untersuchungszeitraum hinweg im Glaszustand sind.
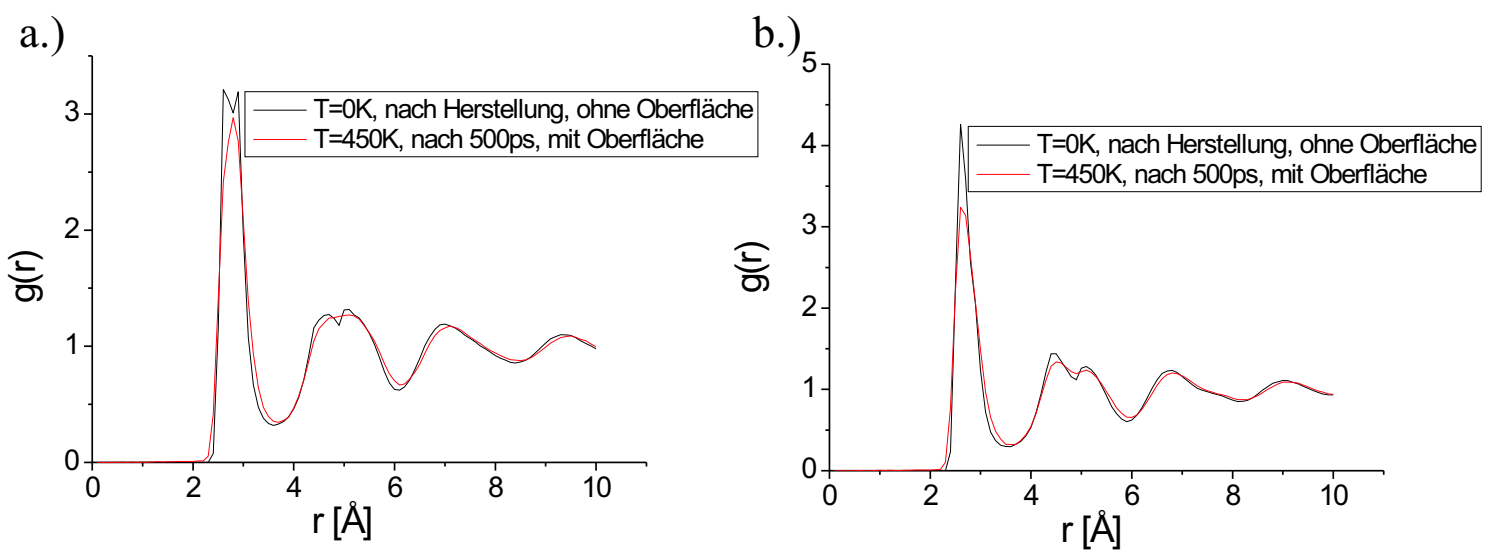

Abbildung 6.3: Paarkorrelationsfunktion von a.) $\mathrm{Cu}_{40} \mathrm{Ti}_{60}$ und b.) $\mathrm{Cu}_{60} \mathrm{Ti}_{40}$ jeweils direkt nach dem Abschreckprozess bei $T=0 \mathrm{~K}$ ohne Oberflächen (schwarze Linie) und nach Abschluss der Diffusionsuntersuchungen über 500 ps bei $T=450 \mathrm{~K}$ mit Oberflächen in einer Richtung (rote Linie). 


\subsubsection{Diffusionsprofile der CuTi Schichten}

Begriffe und Erwartungen Nachdem im vorangegangenen Abschnitt gezeigt wurde, dass glasartiges CuTi in der Simulation hergestellt wurde (siehe auch Diskussion in Kap. 6.2.1), soll nun auf die Dynamik in dünnen Filmen dieses Glases eingegangen werden. Die wesentliche Größe zur Beschreibung der atomaren Dynamik ist der Diffusionskoeffizient. Genauer gesagt soll im Folgenden der Koeffizient der Selbstdiffusion betrachtet werden. Da die hier untersuchten Proben homogen sind, gibt es kein Konzentrationsgefälle, das eine Diffusion in eine bestimmte Richtung verursacht. Als thermischen Prozess gibt es allerdings immer Brownsche Bewegung, die einer stochastischen Bewegung der Atome entspricht. Diese Bewegung führt dazu, dass zwar die mittlere Verschiebung eines Atoms null aber die mittlere quadratische Verschiebung ungleich null ist. Im Falle dreidimensionaler Bewegung gilt für den Diffusionskoeffizienten D [Haa94; KTH91]:

$$
D=\frac{\left\langle\left(\Delta \overrightarrow{r_{i}}\right)^{2}\right\rangle}{6 \Delta t} .
$$

Dabei ist $\Delta \overrightarrow{r_{i}}$ die Verschiebung des mit i indizierten Atoms im Zeitraum $\Delta t$ und \langle\rangle beschreibt die Mittelung über alle durch i indizierten Atome. Eigentlich ist der Diffusionskoeffizient gemäß Gleichung (6.2) im Grenzfall $\Delta t \rightarrow \infty$ definiert. Dieser Grenzfall kann in der Simulation prinzipiell nicht realisiert werden. Hinzu kommt, dass man davon ausgehen muss, dass sich der so definierte Diffusionkoeffizient als Funktion der Zeit ändert, da im Glas auf verschiedenen Zeitskalen Relaxationsprozesse stattfinden, die die Dynamik bestimmen [FFM ${ }^{+} 03$; KS04; Tei05]. Trotzdem ist der in Gleichung 6.2 für endliche Zeitintervalle $\Delta t$ definierte Diffusionskoeffizient ein Maß für die atomare Mobilität und wird daher im Folgenden verwendet. Die Gleichung (6.2) ist außerdem nur dann richtig, wenn sich die Gesamtprobe in Ruhe befindet, d.h die Summe aller atomaren Verschiebungen null ergibt. Falls die Probe jedoch in Bewegung ist, muss als allgemeinere Form

$$
D=\frac{\left\langle\left(\Delta \overrightarrow{r_{i}}-\left\langle\Delta \overrightarrow{r_{i}}\right\rangle\right)^{2}\right\rangle}{6 \Delta t}
$$

verwendet werden. Für die in Kapitel 6 enthaltenen Ergebnisse liefern beide Ausdrücke gleiche Ergebnisse, weil die Proben sich nicht merklich bewegen.

Weil in dieser Arbeit gerade die Oberflächendynamik im Vordergrund steht, ist es sinnvoll einen Diffusionskoeffizienten in Abhängigkeit von der Entfernung des Atoms zur Oberfläche zu berechnen. Dabei sollen ähnliche Methoden wie in [BT99; Böd99] verwendet werden. In der Geometrie der Simulationszelle liegt die z-Achse in der Richtung der Oberflächennormalen. Es werden jeweils die Atome, die zu Beginn in einem bestimmten z-Intervall liegen, zusammengefasst und für die Berechnung des entsprechenden Diffusionskoeffizienten verwendet. Dadurch kann eine unterschiedliche Mobilität in Abhängigkeit von der Entfernung zur Oberfläche sichtbar gemacht werden. Das funktioniert allerdings nur, wenn $\Delta t$ nicht so groß ist, dass sich viele Atome von ihrer Ausgangsposition so weit entfernen, dass sie im Verlauf der Zeit ihren Abstand zur Oberfläche deutlich ändern. In diesem Abschnitt wird als Zeitintervall für die Berechnung des 
Diffusionskoeffizienten $\Delta t=500$ ps gewählt. Der tiefenaufgelöste Diffusionskoeffizient berechnet sich dann analog zum Vorgehen in [BT99; Böd99] gemäß

$$
D(z)=\frac{\sum_{i, z_{i}(t=0) \in[z-\Delta z / 2, z+\Delta z / 2]}\left(\Delta \vec{r}_{i}\right)^{2}}{6 N_{z} \Delta t}
$$

Dabei ist $N_{z}$ die Anzahl der Atome in dem entsprechenden z-Intervall. Neben einer Abhängigkeit von der Entfernung zur Oberfläche muss man für eine Legierung auch eine Abhängigkeit des Diffusionskoeffizienten von der Atomsorte erwarten. Deshalb werden die Diffusionskoeffizienten getrennt für $\mathrm{Cu}$ und Ti Atome berechnet und mit $D^{C u}(z)$ bzw. $D^{T i}(z)$ bezeichnet. In der Berechnung werden dann bei der Summation in Gleichung (6.4) nur die Atome der entsprechenden Sorte verwendet und $N_{z}$ wird dementsprechend durch die Anzahl der Atome der entsprechenden Sorte im jeweiligen z-Intervall ersetzt.

Tiefenaufgelöste Diffusionsprofile für dünne Filme metallischer Gläser wurden bereits für NiZr Schichten bestimmt [BT99; Böd99]. In den zitierten Arbeiten wurde ein exponentieller Anstieg des über beide Atomsorten gemittelten Diffusionkoeffizienten im Oberflächenbereich gefunden, der dort mit einer Landautheorie der Phasenübergänge, angewendet auf den Einfluss von Oberflächen, erklärt werden konnte. Nun sollen aber für dünne Schichten von CuTi Gläsern neben dem Einfluss der Oberfläche erstmals auch das unterschiedliche Verhalten der zwei Atomsorten sowie der Einfluss einer veränderten Zusammensetzung der Legierung untersucht werden.

Ergebnisse Die Proben der Zusammensetzungen $\mathrm{Cu}_{40} \mathrm{Ti}_{60}, \mathrm{Cu}_{50} \mathrm{Ti}_{50}$ und $\mathrm{Cu}_{60} \mathrm{Ti}_{40}$ sind wie in in Kap. 3.4 beschrieben hergestellt und in Abschnitt 6.1.1 charakterisiert. Nach dem Abkühlen aus der Schmelze werden alle Proben bei gleicher Temperaturrate $(20 \mathrm{~K} / \mathrm{ps})$ auf $T=450 \mathrm{~K}$ geheizt, so dass sich entsprechend Kap. 6.1.1 alle Proben unterhalb der Glastemperatur befinden. Wie in Kap. 3.4 beschrieben werden dann Proben mit zwei Oberflächen hergestellt. Die Relaxationszeit der Proben bei $T=450 \mathrm{~K}$ beträgt hier 70 ps, um die wesentlichen Relaxationsprozesse, die sich aufgrund der Änderungen am System durch Erzeugung der Oberflächen ergeben, abzuwarten. Die darauf folgenden 500 ps werden für die Auswertung der Diffusionskoeffizienten verwendet.

Zunächst wird geprüft, ob sich die chemischen Zusammensetzungen der Legierungen an den Probenoberflächen im Vergleich zum Probeninneren ändern, da eine Oberflächensegregation nicht von vornherein auszuschließen ist. Dazu dient das Verhältnis der Anzahl von Atomen der zwei Sorten für alle z-Intervalle der Größe $\Delta z=1 \AA$, welches in Abb. 6.4 gezeigt ist. Die Oberflächen der Proben befinden sich jeweils ungefähr bei $z= \pm 40 \AA$ A. Die äußeren Intervalle werden dann nicht berücksichtigt, wenn die Anzahl der darin enthaltenen Atome sehr klein $(<10)$ ist, da in diesem Fall der statistische Fehler sehr groß wäre. In dem Graphen wird deutlich, dass die Zusammensetzung zwar von Intervall zu Intervall erkennbaren Schwankungen unterliegt, aber kein deutlicher Einfluss der Oberflächen sichtbar ist, wenngleich die Schwankungen in Oberflächennähe etwas größer sind. Allenfalls ist eine Oszillation, aber keine monotone Zu- oder Abnahme eines Elements, zu erkennen. Es lässt sich festhalten, dass es keine starken Änderungen in der 


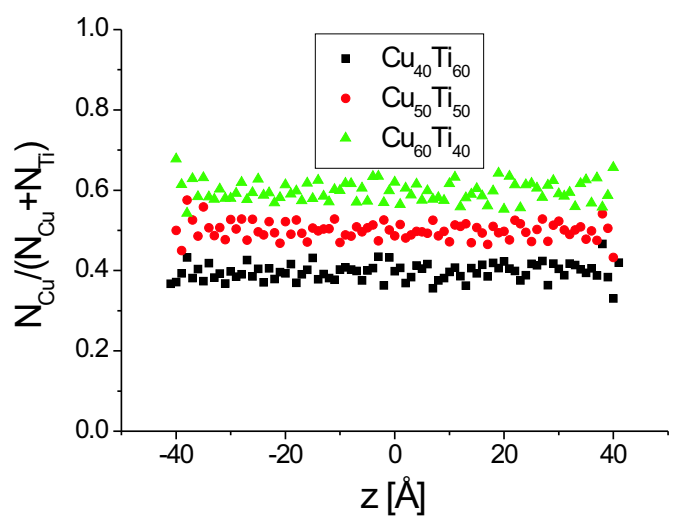

Abbildung 6.4: Tiefenprofile der Zusammensetzung der CuTi Schichten.

Zusammensetzung in Abhängigkeit von der Entfernung zur Oberfläche gibt, wenngleich für genauere Aussagen, die statistischen Fluktuationen zu groß sind.

Mit einer Intervallgröße von $\Delta z=1 \AA$ werden für $\Delta t=500 \mathrm{ps}$ die Diffusionskoeffizienten für $\mathrm{Cu}$ und Ti einzeln berechnet. Die Ergebnisse hierzu sind in Abb. 6.5 aufgetragen. Im Probeninneren befinden sich ca. 220 Atome jeder Sorte in jedem z-Intervall der Breite $1 \AA$. Für die Oberfläche werden die äußeren Intervalle wiederum nicht verwendet, falls sich dort weniger als 10 Atome befinden, weil dann die statistischen Schwankungen zu groß wären. Dieses Kriterium bleibt hier das gleiche wie bei der zuvor besprochenen Untersuchung der Zusammensetzung. Hier sind zunächst zwei Effekte deutlich zu erkennen. Zum einen gibt es an den Oberflächen der Proben einen Anstieg des Diffusionskoeffizienten. An den Oberflächen liegt der Diffusionskoeffizient um etwa eine Größenordnung über dem Wert im Inneren der Probe. Dieser Anstieg betrifft nur einen schmalen Bereich der Probe, nämlich jeweils ungefähr die äußeren $10 \AA$. In der Mitte sind die Diffusionskoeffizienten abgesehen von statistischen Schwankungen über einen Bereich von mindestens $40 \AA$ konstant. Nach dieser Einteilung gibt es noch einen etwa $10 \AA$ breiten Übergangsbereich zwischen Probeninnerem und Oberflächenbereich, in dem der Einfluss der Oberfläche sehr schwach einzusetzen beginnt. Zum anderen liegen die für die $\mathrm{Cu}$ Atome berechneten Werte immer über denen für Ti. Dieser Unterschied zwischen den Elementen wird nicht durch die Oberflächen beeinflusst. Dass der Diffusionskoeffizient in allen analysierten Zusammensetzungen für $\mathrm{Cu}$ Atome größer als für Ti ist, erkennt man auch, wenn man die über die Gesamtprobe gemittelten Werte betrachtet (siehe Tab. 6.2). Aus diesen über die gesamte Probe gemittelten Werten ergibt sich außerdem die Tendenz, dass die Diffusionskoeffizienten für beide Elemente mit zunehmendem Kupferanteil in der Probe abnehmen.

Der Anstieg der einzelnen Diffusionskoeffizienten für $\mathrm{Cu}$ und Ti lässt sich sich sehr gut durch ein exponentielles Verhalten beschreiben, wie es bereits in der Vergangenheit für den gemittelten Diffusionskoeffizienten in gläsernem NiZr gezeigt werden konnte [BT99; Böd99]. Die in Abb. 6.5 eingetragenen Linien entsprechen einem Fit der Daten gemäß 

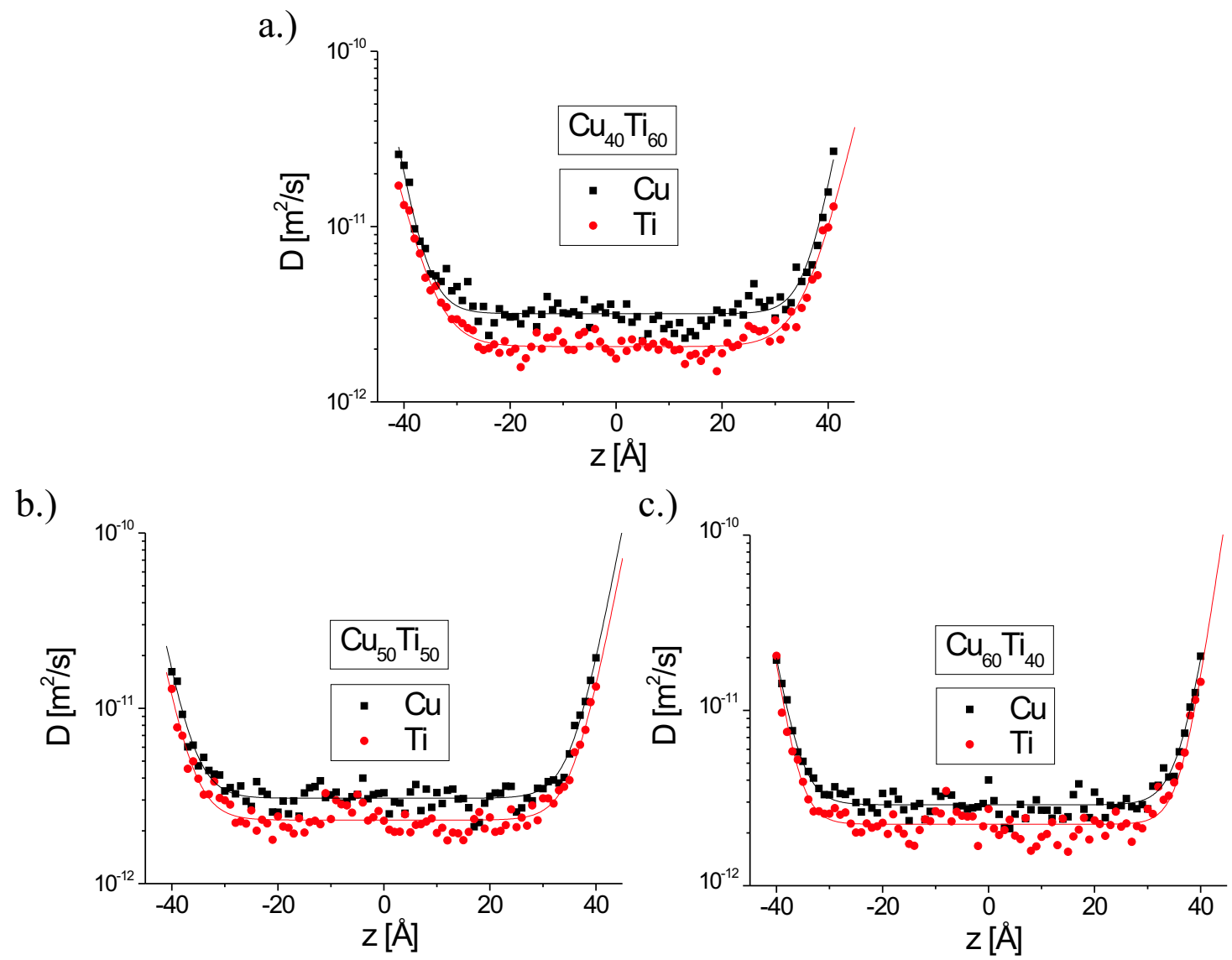

Abbildung 6.5: Tiefenprofile der Diffusionskoeffizienten der CuTi Schichten: a.) $\mathrm{Cu}_{40} \mathrm{Ti}_{60}$, b.) $\mathrm{Cu}_{50} \mathrm{Ti}_{50}$ und c.) $\mathrm{Cu}_{60} \mathrm{Ti}_{40}$.

einer Funktion der Form

$$
D(z)=D_{\infty}\left(1+A e^{-\left(z-d_{l}\right) / z_{0}}+A e^{-\left(d_{r}-z\right) / z_{0}}\right)
$$

wie sie auch in [BT99; Böd99] verwendet wird. Dabei beschreibt $D_{\infty}$ den Diffusionskoeffizienten weit weg von einer Oberfläche, $d_{l}$ bzw. $d_{r}$ sind die Positionen der Oberflächen, $z_{0}$ ist ein Maß für die Breite des Bereichs, in dem ein durch die Oberfläche induzierter Anstieg stattfindet, und $A$ ist ein Maß für die Höhe dieses Anstiegs. Diese Gleichung kann man entweder als empirisch begründete Gleichung auffassen oder aus einem Landaumodell für Phasenübergänge, das sich auch auf eingeschränkte Geometrien ausdehnen lässt, herleiten. Dieser Zusammenhang ist in [BT99; Böd99] ausführlich beschrieben. Gleichung (6.5) lässt sich noch etwas umordnen, so dass man erkennt, dass tatsächlich nur vier unabhängige Parameter zur Verfügung stehen:

$$
D(z)=D_{\infty}\left(1+A e^{d_{l} / z_{0}}\left(e^{-z / z_{0}}+e^{-\left(d_{l}+d_{r}-z\right) / z_{0}}\right)\right) .
$$

Die an dieser Stelle für die Probencharakterisierung wichtigen Parameter sind $D_{\infty}$ als Maß für den Diffusionkoeffizienten im Probeninneren und $z_{0}$ als Maß für den Einflussbe- 


\begin{tabular}{|l|l|l|l|}
\hline & $\mathrm{Cu}_{40} \mathrm{Ti}_{60}$ & $\mathrm{Cu}_{50} \mathrm{Ti}_{50}$ & $\mathrm{Cu}_{60} \mathrm{Ti}_{40}$ \\
\hline$D^{C u}\left[10^{-12} \cdot \mathrm{m}^{2} / \mathrm{s}\right]$ & $4.51 \pm 0.03$ & $4.17 \pm 0.06$ & $3.78 \pm 0.05$ \\
\hline$D^{T i}\left[10^{-12} \cdot \mathrm{m}^{2} / \mathrm{s}\right]$ & $3.13 \pm 0.04$ & $3.08 \pm 0.05$ & $2.92 \pm 0.05$ \\
\hline
\end{tabular}

Tabelle 6.2: Diffusionskoeffizienten für $\mathrm{Cu}$ und Ti bei verschiedenen Zusammensetzungen als Mittelung über die gesamte Probe.

\begin{tabular}{|l|l|l|l|}
\hline & $\mathrm{Cu}_{40} \mathrm{Ti}_{60}$ & $\mathrm{Cu}_{50} \mathrm{Ti}_{50}$ & $\mathrm{Cu}_{60} \mathrm{Ti}_{40}$ \\
\hline$D_{\infty}^{C u}\left[10^{-12} \cdot \mathrm{m}^{2} / \mathrm{s}\right]$ & $3.19 \pm 0.11$ & $3.07 \pm 0.07$ & $2.89 \pm 0.06$ \\
\hline$D_{\infty}^{T i}\left[10^{-12} \cdot \mathrm{m}^{2} / \mathrm{s}\right]$ & $2.07 \pm 0.06$ & $2.30 \pm 0.05$ & $2.24 \pm 0.07$ \\
\hline$z_{0}^{C u}[\AA]$ & $2.55 \pm 0.12$ & $2.74 \pm 0.11$ & $2.42 \pm 0.08$ \\
\hline$z_{0}^{T i}[\AA]$ & $3.42 \pm 0.12$ & $2.78 \pm 0.13$ & $2.13 \pm 0.11$ \\
\hline
\end{tabular}

Tabelle 6.3: Fitparameter $D_{\infty}$ und $z_{0}$ aus Gleichung (6.6) für $\mathrm{Cu}$ und Ti bei verschiedenen Zusammensetzungen.

reich der Oberfläche. Die entsprechenden Werte sind in Tab 6.3 eingetragen. Die Werte für $D_{\infty}$ zeigen wiederum, dass die $\mathrm{Cu}$ Atome im Probeninneren einen höheren Diffusionskoeffizienten als die Ti Atome aufweisen.

\subsubsection{Verteilungsfunktion atomarer Verschiebungen}

Begriffe und Erwartungen Um Aussagen über die Mechanismen atomarer Dynamik treffen zu können, kann die Analyse der Verteilungsfunktion atomarer Verschiebungen hilfreich sein. Diese Verteilungsfunktion beschreibt die Wahrscheinlichkeit eines Atoms, in der vorgegebenen Zeit eine Verschiebung bestimmter Größe zu erfahren. Es werden also die Positionen der einzelnen Atome zu verschiedenen Zeitpunkten analysiert. Im isotropen System sind wiederum nur die Beträge der Verschiebungen von Interesse, so dass die Verteilung als Funktion dieser Größe dargestellt werden soll. Daher berechnet man mit der Diracschen $\delta$-Funktion

$$
G(r, t)=\frac{1}{N_{p}} \sum_{i=1}^{N_{p}}\left\langle\delta\left(\left|\overrightarrow{r_{i}}(t)-\overrightarrow{r_{i}}(0)\right|-r\right)\right\rangle_{\Delta r}
$$

als Histogramm der auftretenden Verschiebungsweiten mit einer Intervallbreite $\Delta r$, wobei \langle\rangle$_{\Delta r}$ eine räumliche Mittelung im Intervall $[r-\Delta r / 2, r-\Delta r / 2]$ bedeutet, d.h. $\left\langle\delta\left(\left|\overrightarrow{r_{i}}(t)-\overrightarrow{r_{i}}(0)\right|-r\right)\right\rangle_{\Delta r}=\frac{1}{\Delta r} \int_{r-\Delta r / 2}^{r+\Delta r / 2} \delta\left(\left|\overrightarrow{r_{i}}(t)-\overrightarrow{r_{i}}(0)\right|-r^{\prime}\right) d r^{\prime} . N_{p}$ ist die Anzahl der Atome, die in die Summation eingehen. Diese Summation läuft je nach Bedarf über diejenigen Atome, die bezüglich ihrer Atomsorte und z-Koordinate die gewünschten Bedingungen erfüllen. Falls im betrachteten System Sprünge diskreter Weiten auftreten, ergeben sich im Histogramm an den entsprechenden Stellen Maxima. Für eine kooperative Dynamik, an der eine Vielzahl von Atomen beteiligt ist, erwartet man dagegen eine Gaußverteilung der Verschiebungen [Cra75; Wah91]. Die Gaußverteilung wird allgemein für eine kooperative Dynamik erwartet, unabhängig davon ob es sich um Transport in 
a.)

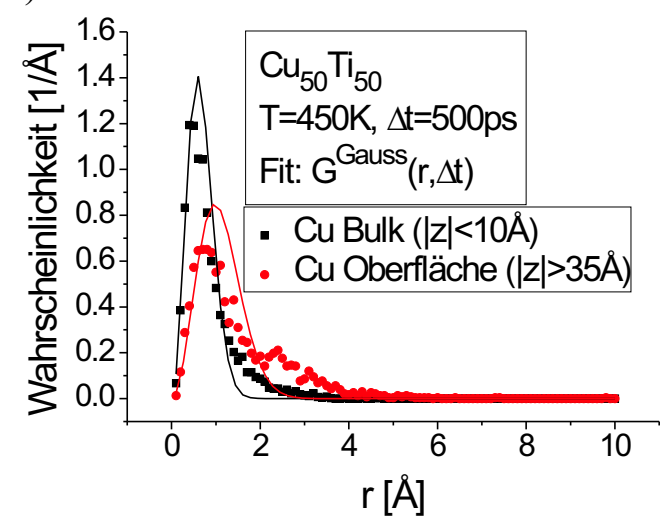

b.)

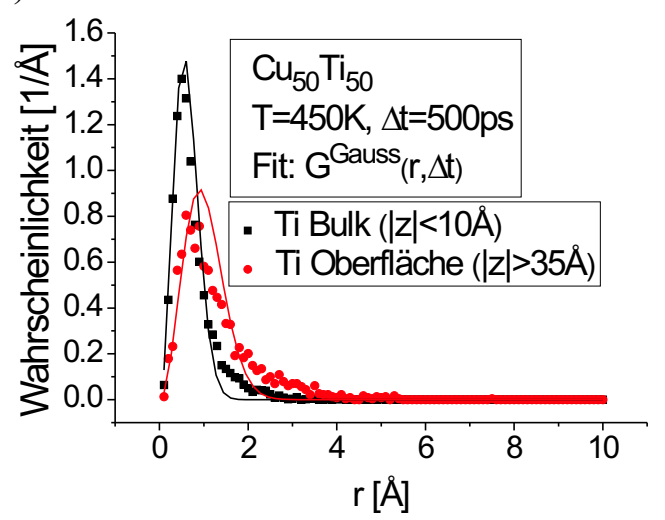

Abbildung 6.6: Verteilung atomarer Verschiebungen in $\mathrm{Cu}_{50} \mathrm{Ti}_{50}$ im Probeninneren und im Oberflächenbereich für a.) $\mathrm{Cu}$ und b.) Ti.

einer Flüssigkeit im hydrodynamischen Grenzfall oder um thermische Schwingungen im eingefrorenen Zustand handelt [Wah91]. Bei einer radialen Mittelung im dreidimensionalen Fall bedeutet das für den hydrodynamischen Grenzfall

$$
G^{\mathrm{Gauß}}(r, t)=\frac{4 \pi r^{2}}{(4 \pi D t)^{3 / 2}} \exp \left(-r^{2} / 4 D t\right)
$$

mit dem Diffusionskoeffizienten $D$. Die mathematische Analyse dieser Gleichung liefert die Erkenntnis, dass ein Maximum an der Position $r_{\max }=\sqrt{4 D t}$ existiert. Für thermische Schwingungen in einem eingefrorenen System ist der Kurvenverlauf im Prinzip gleich mit dem Unterschied, dass anstatt der Parameter $D$ und $t$ die Temperatur und eine Materialkonstante in die Formel eingehen. Damit ändert sich mathematisch nichts an der anzupassenden Funktion. Die physikalische Interpretation ist allerdings eine andere. Gemäß der verwendeten Definitionen liefern sowohl $G$ als auch $G^{\text {Gauß }}$ Wahrscheinlichkeiten für eine bestimmte Verschiebungsweite mit einer reziproken Längeneinheit als Einheit.

Ergebnisse In Kap. 6.1.2 hat sich anhand der Diffusionsprofile gezeigt, dass im Inneren der Probe die Atome ein bulk-ähnliches, d.h. nicht von Oberflächen beeinflusstes, Verhalten zeigen, während sich Abweichungen hierzu im Bereich der Oberflächen ergeben. Das bulk-ähnliche Verhalten gilt sicherlich für Atome, für deren z-Koordinate $|z|<10 \AA$ erfüllt ist. Solche Atome bilden den Bereich, der im Folgenden als Inneres der Probe bezeichnet wird. Als Oberflächenatome werden hingegen solche bezeichnet, für die $|z|>35 \AA$ gilt, da für diese in den Diffusionsprofilen der Oberflächeneinfluss deutlich ausgeprägt ist.

Die $\mathrm{Cu}_{50} \mathrm{Ti}_{50}$ Probe wird nun gemäß Gleichung (6.7) getrennt für $\mathrm{Cu}$ bzw. Ti Atome im Inneren der Probe und im Oberflächenbereich ausgewertet. Die Ergebnisse sind in Abb. 6.6 als Punkte dargestellt und die durchgezogenen Linien stellen Fits der Gleichung (6.8) an die Ergebnisse dar. Im Probeninneren lassen sich sowohl für die $\mathrm{Cu}$ 
als auch für die Ti Atome die Ergebnisse relativ gut mit der Gaußverteilung gemäß Gleichung (6.8) beschreiben. Im Vergleich zum Inneren der Probe ist für Atome aus dem Oberflächenbereich bei beiden Atomsorten die Verteilung der atomaren Verschiebungen deutlich zu größeren Werten verschoben. Es gibt also deutlich mehr weite atomare Verschiebungen auf Kosten der kürzeren. Dieser Unterschied entspricht zunächst der Zunahme der Mobilität für beide Atomsorten, die auch schon in Abb. 6.5 sichtbar ist. Ein weiterer Unterschied im Verhalten der Oberflächenatome gegenüber denen im Probeninneren liegt darin, dass sich die Ergebnisse weniger gut durch die Gaußverteilung beschreiben lassen. Neben dem ersten Maximum bildet sich im Falle von Ti eine deutliche Schulter heraus, während beim $\mathrm{Cu}$ sogar die Ausbildung eines zweiten Maximums sichtbar ist. Dieses zweite Maximum für $\mathrm{Cu}$ Oberflächenatome liegt ungefähr bei $r=2.4 \AA$ und damit sehr nahe am Wert für den Abstand nächster Nachbarn in Kupfer, der laut [Kit93] bei $a_{C u}=2.56 \AA$ Aliegt. Das zusätzliche Maximum ist nicht sehr scharf, sondern eher breit um den Abstand nächster Nachbarn herum verteilt.

Um weiter zu klären, worin die Unterschiede zwischen dem Probeninneren und dem Oberflächenbereich bestehen, sollen im folgenden Abschnitt die Verteilungen der Energie der Atome untersucht werden.

\subsubsection{Energieverteilung}

Für den Zusammenhang der atomaren Dynamik mit der lokalen atomaren Umgebung der sich bewegenden Atome ist die potentielle Energie dieser Atome von großer Bedeutung, da sich diese gerade aus den Bindungen zu benachbarten Atomen ergibt (siehe Kap. 3.3.2.1). Die Verteilung der potentiellen Energie ist für alle $\mathrm{Cu}$ und alle Ti Atome der $\mathrm{Cu}_{50} \mathrm{Ti}_{50}$ Probe mit Oberflächen bei $T=450 \mathrm{~K}$ nach Abschluss der Diffusionsuntersuchungen in Abb. 6.7 dargestellt. Mit eingetragen ist jeweils eine angepasste Gauß-Normalverteilung mit zwei variablen Parametern, die zum einen der Position des Maximums auf der Ordinate $E_{M}$ und zum anderen der die Breite der Verteilung charakterisierenden Standardabweichung $\sigma_{E}$ entsprechen. Der Wert im Maximum ist durch die bekannte Anzahl von Atomen und die verwendete Intervallbreite bereits festgelegt.

Bei den $\mathrm{Cu}$ Atomen fällt eine deutliche Schulter im Bereich hoher (also vom Betrag her kleiner) Energien auf. Bei kleinen Energien gibt es eine solche Schulter nicht, so dass die Daten insgesamt eine asymmetrische Verteilung liefern. Der Grenzwert, ab dem die Abweichung bei hohen Energien deutlich wird, liegt ungefähr bei dem Wert, der sich aus $E_{M}+2 \sigma_{E}$ ergibt. Als Orientierungshilfe ist deshalb bei $E_{M} \pm 2 \sigma_{E}$ im Graphen jeweils eine vertikale Linie eingetragen. Die Asymmetrie der Verteilung wird auch dadurch bestätigt, dass es $1043 \mathrm{Cu}$ Atome mit $E_{\text {pot }}>E_{M}+2 \sigma_{E}$ aber nur $250 \mathrm{Cu}$ Atome mit $E_{\text {pot }}<E_{M}-2 \sigma_{E}$ gibt. Von den $1043 \mathrm{Cu}$ Atomen oberhalb der Grenze $E_{M}+2 \sigma_{E}$ befinden sich 722 im Oberflächenbereich $|z|>35 \AA$. Aus geometrischen Überlegungen lässt sich die Anzahl der Cu Oberflächenatome grob zu $N_{S}^{C u}=2 \cdot \frac{1}{2} \cdot N^{2 / 3}$ abschätzen ( $\mathrm{N}=$ Anzahl von Atomen in der gesamten würfelförmigen Probe, Faktor 2 wegen zwei Oberflächen, Faktor 1/2 entsprechend dem $\mathrm{Cu}$ Anteil). Damit erhält man für die betrachtete Probe $N_{S}^{C u}=1073$. Diese Ergebnisse zeigen zum einen, dass die erkennbare Schulter vorwiegend durch die Oberflächenatome gebildet wird. Zum anderen ist die Anzahl der Cu 
a.)

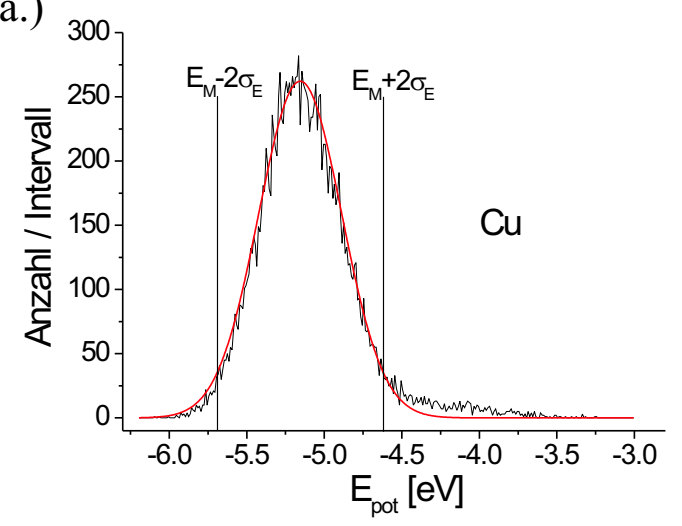

b.)

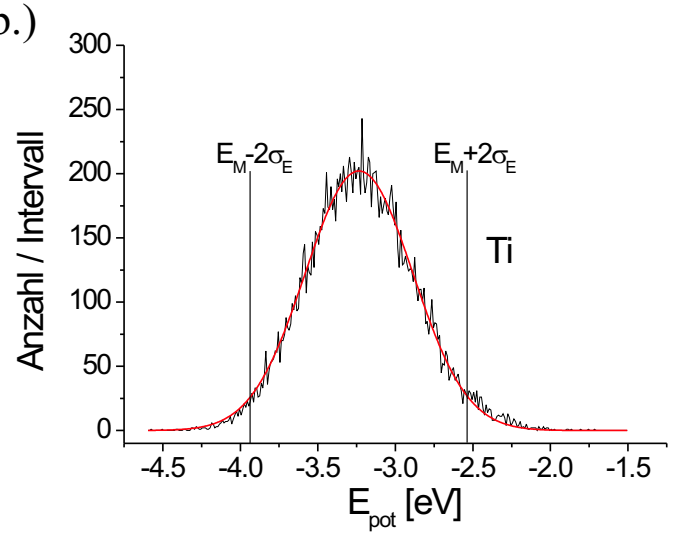

Abbildung 6.7: Verteilung potentieller Energien der einzelnen Atome in der gesamten $\mathrm{Cu}_{50} \mathrm{Ti}_{50}$ Probe bei $\mathrm{T}=450 \mathrm{~K}$ für a.) $\mathrm{Cu}$ und b.) $\mathrm{Ti}$ (jeweils schwarze Linie). Die rote Linie stellt eine angepasste Gauß - Normalverteilung dar.

Atome, die eine erhöhte potentielle Energie aufweisen und sich im Oberflächenbereich befinden, von ähnlicher Größenordnung wie die Gesamtzahl von $\mathrm{Cu}$ Oberflächenatomen. Diese beiden Resultate lassen sich durch eine Projektion der Atome auf die xz-Ebene illustrieren, wie sie in Abb. 6.8 gezeigt ist.

Bei der Verteilung der Ti Atome gibt es eine solch deutliche Asymmetrie in der Verteilung nicht. Allerdings gilt auch hier, dass die Anzahl der Ti Atome mit $E_{p o t}>E_{M}+2 \sigma_{E}$ größer ist als die der Ti Atome mit $E_{p o t}<E_{M}-2 \sigma_{E}$, denn es ergeben sich 557 Ti Atome mit hoher und 306 mit niedriger Energie. Diese leichte Asymmetrie ist auch daran erkennbar, dass die Daten im Bereich hoher Energien etwas oberhalb der angepassten Gaußverteilung und für kleine Energien etwas darunter liegen. Für die Ti Atome, die $E_{\text {pot }}>E_{M}+2 \sigma_{E}$ erfüllen, kann man allerdings nicht feststellen, dass sich diese vorwiegend im Oberflächenbereich befinden. Diese Ti Atome sind über die gesamte Probe relativ gleichmäßig verteilt, wie in Abb. $6.8 \mathrm{zu}$ sehen ist. Quantitativ ergibt sich, dass von den $557 \mathrm{Ti}$ Atomen, die $E_{\text {pot }}>E_{M}+2 \sigma_{E}$ erfüllen, sich nur 72 im Oberflächenbereich $|z|>35 \AA$ befinden. Damit ist die Dichte von Ti Atomen mit erhöhter Energie im Oberflächenbereich gegenüber der Gesamtprobe nicht wesentlich erhöht.

Der Vergleich der beiden Atomsorten zeigt außerdem, dass die durchschnittliche potentielle Energie der Ti Atome größer ist als die der $\mathrm{Cu}$ Atome $\left(E_{M}^{C u}=(-5.155 \pm 0.002) \mathrm{eV}\right.$, $\left.E_{M}^{T i}=(-3.234 \pm 0.002) \mathrm{eV}\right)$. Weiterhin liefert eine Analyse der Proben mit anderen Zusammensetzungen, dass die Energie für beide Atomsorten ansteigt, wenn der $\mathrm{Cu}$ Anteil zunimmt. Es ergibt sich nämlich für $\mathrm{Cu}_{40} \mathrm{Ti}_{60} E_{M}^{C u}=(-5.413 \pm 0.002) \mathrm{eV}$ bzw. $E_{M}^{T i}=(-$ $3.589 \pm 0.002) \mathrm{eV}$ und für $\mathrm{Cu}_{60} \mathrm{Ti}_{40} E_{M}^{C u}=(-4.884 \pm 0.002) \mathrm{eV}$ bzw. $E_{M}^{T i}=(-2.875 \pm$ $0.002) \mathrm{eV}$. Das qualitative Verhalten mit der Existenz einer Schulter bei hohen Energien für $\mathrm{Cu}$ Atome und einer recht guten Übereinstimmung mit einer Gaußverteilung für Ti Atome ist bei allen untersuchten Zusammensetzungen gleich. 

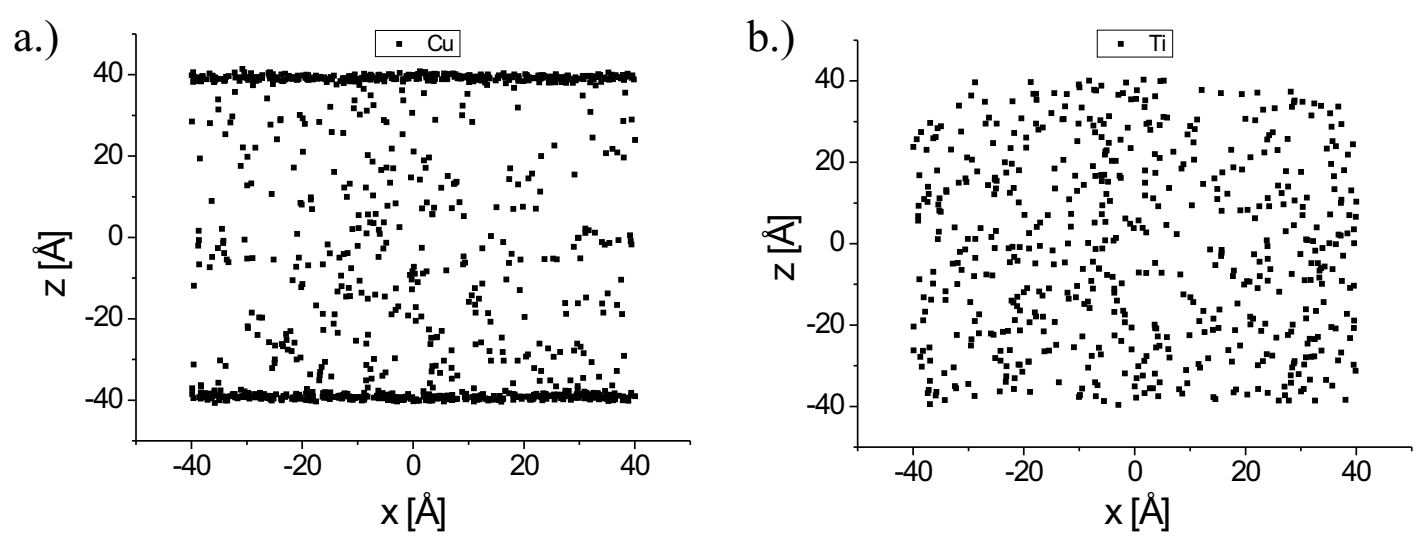

Abbildung 6.8: Positionen der Atome mit potentieller Energie $E_{\text {pot }}>E_{M}+2 \sigma_{E}$ als Projektion auf die xz-Ebene in der $\mathrm{Cu}_{50} \mathrm{Ti}_{50}$ Probe bei $T=450 \mathrm{~K}$ für a.) $\mathrm{Cu}$ und b.) $\mathrm{Ti}$.

\subsubsection{Atomare Dynamik bei unterschiedlichen Verschiebungsweiten}

Um weiter zu klären, wie sich Atome, die sich relativ weit bewegen, von solchen mit einer kleinen Verschiebung unterscheiden, sollen in diesem Abschnitt beispielhaft Vertreter entsprechender Atome betrachtet werden. Es soll gezeigt werden, was die Unterschiede und Gemeinsamkeiten von Atomen, die während der Simulation eine kurze bzw. eine weite Strecke zurücklegen, sind. Dazu werden beispielhaft zwei $\mathrm{Cu}$ Atome aus dem Oberflächenbereich der Probe betrachtet. Atom 1 legt eine weite Strecke zurück und Atom 2 eine kurze.

Die zugehörigen Trajektorien sind in Abb. 6.9 mit einer Zeitschrittweite von $\Delta t=5 \mathrm{ps}$ dargestellt. Während Atom 2 für die gesamte Zeit in der Nähe seines Ursprungsortes bleibt, wechselt Atom 1 zweimal den Aufenthaltsbereich, nachdem es sich dort jeweils für längere Zeit befunden hat. Dieses Verhalten wird auch durch die Auftragung der Varianz als Funktion der Zeit in Abb. 6.10.a deutlich. Nach einer ersten Bewegung zum Beginn der Simulation sieht man, dass Atom 1 den Aufenthaltsort bei ca. 110 ps und bei ca. 310 ps deutlich ändert. Die Änderung der Varianz in der Nähe der Zeit 110 ps entspricht außerdem ungefähr der Änderung, die ein Sprung der Länge des Abstandes nächster Nachbarn bewirkt. Bildet man den Mittelwert der Beträge der atomaren Verschiebungen für die Zeitintervalle von $\Delta t=5 \mathrm{ps}$, so zeigt sich, dass dieser Wert für beide Atome ungefähr gleich ist (Atom 1: $\frac{1}{100} \sum_{i=1}^{100}|\vec{r}(t=i \Delta t)-\vec{r}(t=(i-1) \Delta t)|=0.48 \AA$, Atom 2: $\left.\frac{1}{100} \sum_{i=1}^{100}|\vec{r}(t=i \Delta t)-\vec{r}(t=(i-1) \Delta t)|=0.55 \AA\right)$. Insbesondere ist der Wert also nicht größer für das Atom, das sich insgesamt weiter bewegt. Das bedeutet, dass beide Atome sozusagen immer in Bewegung sind. Der Unterschied beider Atome besteht aber in der Richtung der Verschiebungen. Außerdem liegt die Verschiebungslänge zu den Zeitpunkten, an denen das Atom den Aufenthaltsbereich wechselt, deutlich über dem Durchschnittswert.

Um Informationen über die Dynamik in der Probe zu gewinnen, spielt neben der 
a.)

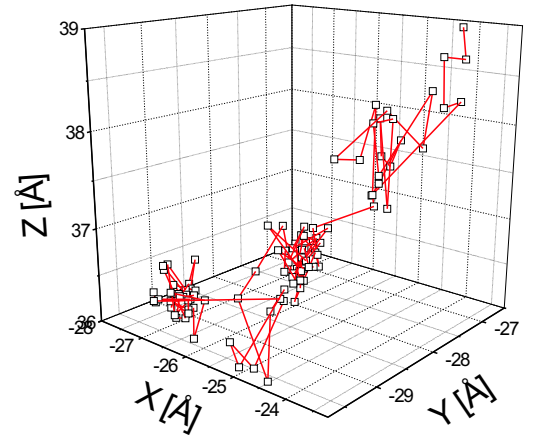

b.)

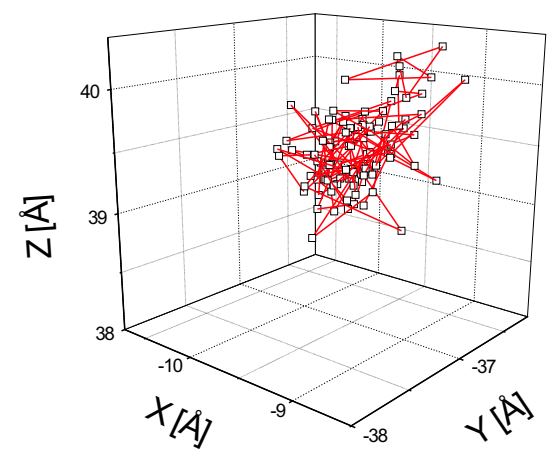

Abbildung 6.9: Trajektorien für a.) Atom 1 und b.) Atom 2.
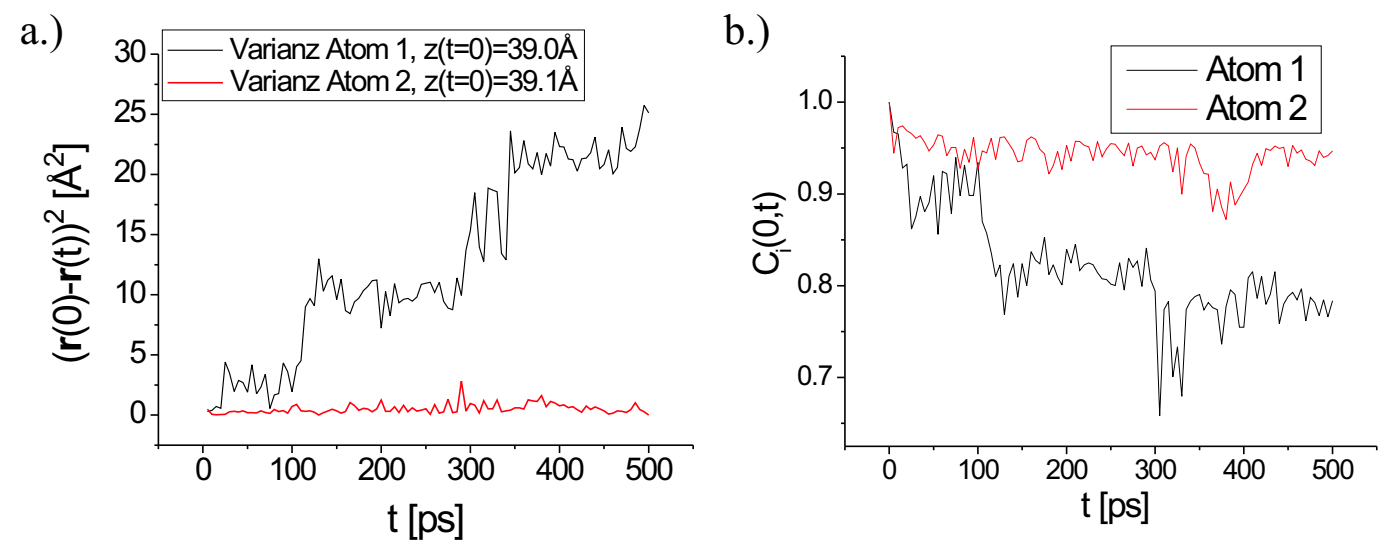

Abbildung 6.10: a.) Varianz als Funktion der Zeit für Atom 1 und Atom 2, b.) NachbarKorrelationsfunktion für Atom 1 und Atom 2 für die Zeitpunkte $t_{1}=0$ und $t_{2}=t$.

beschriebenen absoluten Bewegung einzelner Atome auch eine wesentliche Rolle, wie sich diese Atome relativ zu ihren Nachbarn bewegen. Es stellt sich also die Frage, ob sich das betrachtete Atom während der Simulation von seinen Nachbarn zum Teil trennt, oder ob die Nachbarn beibehalten werden. Über diese Frage kann eine NachbarKorrelationsfunktion Aufschluss geben, durch die die Nachbarn zu zwei Zeitpunkten $t_{1}$ und $t_{2}$ verglichen werden. Eine solche Funktion lässt sich ähnlich wie in [Tei92] hier für ein festgelegtes Bezugsatom $i$ folgendermaßen definieren:

$C_{i}\left(t_{1}, t_{2}\right)=\frac{\sum_{j=1}^{N} B_{i, j}\left(t_{1}\right) B_{i, j}\left(t_{2}\right)}{\left(\sum_{j=1}^{N} B_{i, j}\left(t_{1}\right) \sum_{j=1}^{N} B_{i, j}\left(t_{2}\right)\right)^{1 / 2}}$ mit $B_{i, j}(t)= \begin{cases}1 & \text { für }\left|\vec{r}_{i}(t)-\vec{r}_{j}(t)\right| \leq r_{c} \\ 0 & \text { sonst }\end{cases}$

Dabei ist $r_{c}$ ein Radius innerhalb dessen die Nachbarn bestimmt werden. Um nur die 


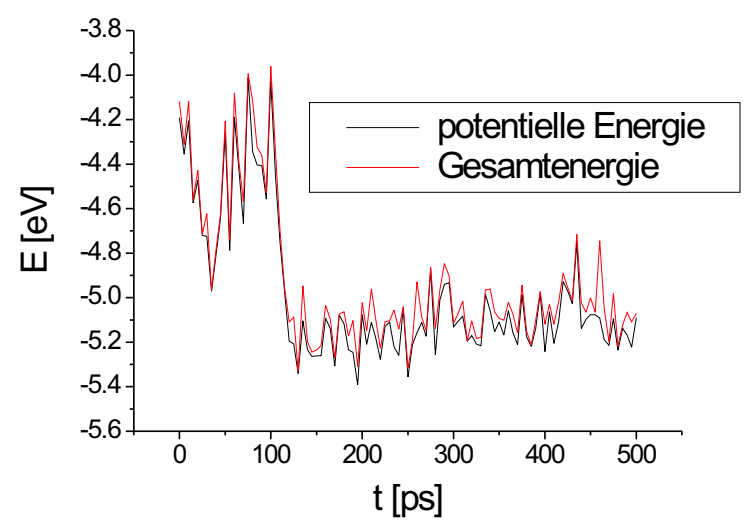

Abbildung 6.11: Potentielle Energie und Gesamtenergie von Atom 1 im Verlauf der Simulation.

Korrelation zu den nächsten Nachbarn zu betrachten, wird der Wert von $r_{c}$ durch die Position des zwischen dem ersten und zweiten Maximum liegenden Minimums in der Paarkorrelationsfunktion aus Abb. 6.2 gewählt. Daraus ergibt sich $r_{c}=3.7 \AA$. Für die zwei zuvor diskutierten Atome ist die Nachbar-Korrelationsfunktion in Abb. 6.10.b dargestellt. Hier ist erkennbar, dass Atom 1 zu den Zeitpunkten, zu denen sich Sprünge in der Varianz ergeben (siehe Abb. 6.10.a), auch jeweils einen Teil seiner ursprünglichen Nachbarn verliert. Für Atom 2 gibt es dagegen nach etwa 350 ps einen reversiblen Verlust von Nachbarn, nach dem der Wert der Korrelationsfunktion wieder auf den zuvor bestehenden Wert zurückkehrt.

Weiterhin stellt sich die Frage, aus welchem Grund Atom 1 sich so weit bewegt. Dafür kommen zwei mögliche Gründe in Frage: Zum einen könnte es durch Zufall im Sinne der "random walk" Bewegung sein und zum anderen könnte es durch einen Gradienten im chemischen Potential bedingt sein. In Abb. 6.11 ist sowohl die potentielle als auch die gesamte Energie von Atom 1 als Funktion der Zeit dargestellt. In diesem Graphen wird deutlich, dass Atom 1 durch die Bewegung bei ca. 110 ps (siehe auch Abb. 6.10.a) seine Energie deutlich absenkt. Dagegen ist bei der Bewegung nach ca. 310 ps keine deutliche Änderung der Energie erkennbar. Bewegung kann also sowohl mit als auch ohne deutliche Änderung der Energie des sich bewegenden Atoms stattfinden, d.h beide oben vorgestellten Möglichkeiten sind hier von Bedeutung.

\subsection{Diskussion der Ergebnisse zum thermisch aktivierten Verhalten von CuTi Schichten}

\subsubsection{Probenherstellung und Charakterisierung}

Zusammenfassend kann man sagen, dass die Ergebnisse aus Kap. 6.1.1 zeigen, dass mit der gewählten Herstellungsmethode, nämlich dem raschen Abkühlen aus der Schmelze, 
ein metallisches Glas hergestellt werden kann.

Die thermodynamischen Größen Volumen und Enthalpie zeigen das für einen Glasübergang erwartete Verhalten, indem sie eine über einen gewissen Temperaturbereich verschmierte Steigungsänderung als Funktion der Temperatur aufweisen. Dass die Übergangstemperaturen weitestgehend unabhängig davon sind, ob sie aus dem Verlauf des Volumens oder der Enthalpie als Funktion der Temperatur bestimmt wurden, entspricht der Erwartung, wie sich aus der Analyse des Zusammenhangs der Zustandsgrößen Volumen und Enthalpie ergibt. Das hier behandelte System entspricht einem Modell positiv geladener Atomrümpfe mit einem Hintergrund elektronischer Ladungsträger. Dort besteht ein linearer Zusammenhang zwischen der Energie und der elektronischen Ladungsdichte, die wiederum umgekehrt proportional zum Probenvolumen ist [PNM81]. Auch der Energiebeitrag der Atomrümpfe ändert sich stetig durch eine Volumenänderung und somit besteht eine stetige Abhängigkeit zwischen Energie und Volumen. Daher werden sich Unstetigkeiten im Volumen auf Unstetigkeiten in der Energie übertragen und umgekehrt. Ähnlich verhält es sich für den 'verschmierten' Knick beim Glasübergang.

Markant ist, dass die Übergangstemperatur während des Heizens deutlich größer als während des Kühlens ist. In der Literatur wird dagegen meist davon ausgegangen, dass beide Übergangstemperaturen zumindest sehr nahe beieinander liegen [Zar91; $\left.\mathrm{FFM}^{+} 03\right]$. Dass im Gegensatz dazu hier die Temperaturen um etwa $160 \mathrm{~K}$ differieren, lässt sich als mit der Art der Temperaturkontrolle und den hohen Raten der Simulation erklären: Eine Temperaturänderung erfolgt nach dem Berendsen-Algorithmus [BPG $\left.{ }^{+} 84\right]$ instantan durch Reskalierung. Dabei wird außerdem immer die aus der kinetischen Energie bestimmte Temperatur ausgewertet und die unterscheidet sich leicht von der in der Thermodynamik definierten Temperatur $T=\partial E / \partial S$ [Bre96]. Die Atome brauchen daher immer noch etwas Zeit, um ihre Konfiguration den thermischen Änderungen anzupassen. Demnach würde die atomare Konfiguration, die sich sowohl im spezifischen Volumen als auch in der Energie pro Atom widerspiegelt, der vorgegebenen Temperatur immer zeitverzögert folgen. Daher ist zu erwarten, dass die beim Abkühlen bestimmte Temperatur zu niedrig und die beim Aufheizen bestimmte Temperatur zu hoch ist. Weil die Kühl- bzw. Heizraten gleich sind, entspräche der tatsächliche Wert gerade dem Mittelwert beider Übergangstemperaturen. Die Zeit, die die Atome benötigen, um sich in ihrer Konfiguration tatsächlich auf die geänderte Temperatur einzustellen, sollte im Bereich einiger thermischer Schwingungen liegen. Die kürzeste Schwingungsdauer im System kann aus Phononenspektren gewonnen werden, die durch Experimente mit inelastischer Neutronenstreuung vermessen werden können. Für metallische Gläser ergibt sich mit dieser Methode $\tau \approx 100$ fs [SGB80; SR83]. Berechnungen von Phonenspektren metallischer Gläser aus MD-Simulationen liefern sehr ähnliche Werte [HK94]. In den Phononenspektren tauchen kontinuierlich weitere Moden bis Schwingungsdauern über 1 ps hinaus auf. Bedenkt man nun, dass die verwendete Kühlrate $20 \mathrm{~K} /$ ps beträgt, wird klar, dass sich die in der Simulation vorgegebene Temperatur während einiger Schwingungen in einer Größenordnung geändert hat, die der Abweichung der Übergangstemperaturen aus Heizund Kühlprozess vom Mittelwert entspricht.

Die bestimmten Übergangstemperaturen sind weitgehend unabhängig von der Zusammensetzung innerhalb des betrachteten Bereichs. Das bedeutet, dass das thermody- 
namische Verhalten sich nicht stark ändert, wenn man die Konzentrationsverhältnisse innerhalb von zehn Prozent variiert.

Ein Vergleich der hier bestimmten Glastemperaturen mit experimentell bestimmten, zeigt im Fall von $\mathrm{Cu}_{50} \mathrm{Ti}_{50}$, dass die Ergebnisse aus dem Experiment und der Simulation nicht sehr weit auseinanderliegen. Je nach Heizrate wird in $\left[\mathrm{KDD}^{+} 93\right]$ aus Messungen der Wärmekapazität ein Bereich $593 \mathrm{~K}<T_{g}<705 \mathrm{~K}$ angegeben, wobei die Heizraten für die untere Grenze $10^{-2} \mathrm{~K} / \mathrm{s}$ und für die obere Grenze $5 \cdot 10^{6} \mathrm{~K} / \mathrm{s}$ sind. Dagegen wird in [KMAB93] für eine Heizrate von $1.3 \mathrm{~K} / \mathrm{s}$ der Wert $T_{g}=690 \mathrm{~K}$ angegeben. Aufgrund der deutlich höheren Heiz- und Kühlraten in der MD-Simulation (siehe Kap. 3.4) wäre für die Simulationsergebnisse zunächst eine höhere Übergangstemperatur zu erwarten, wobei hier aus den oben beschriebenen Gründen stets der Mittelwert aus den Ergebnissen für das Heizen und Abkühlen verwendet werden muss. Gemäß der allgemeinen Erwartung sollte die Glastemperatur nämlich mit sinkender Heiz- bzw. Kühlrate kleiner werden und im Grenzfall unendlich kleiner Rate in die Kauzmann-Temperatur münden [Ell90]. Dies trifft für den Vergleich zwischen experimentellen Daten und Simulationsdaten allerdings nicht zu. Die experimentellen Ergebnisse für die höchsten Heizraten sind sogar größer als die Werte aus der Simulation, obwohl dort deutlich größere Heiz- bzw. Kühlraten verwendet werden. So gesehen zeigen die Simulationen, wenn man den Effekt der unterschiedlichen Raten mit einrechnet, einen Glasübergang bei zu niedrigen Temperaturen. Ein Grund dafür ist wahrscheinlich, dass das verwendete Wechselwirkungspotential keine kovalenten Bindungsanteile beinhaltet, die aber in der Realität durchaus vorhanden sind. Starke kovalente Bindungsanteile zeichnen ein starkes Glas, das je nach Stärke dieser Bindungen eine Netzwerkstruktur eingehen kann, im Gegensatz zu einem fragilen Glas aus. Daher würden zusätzliche kovalente Bindungen die Fähigkeit zur plastischen Deformation verringern und somit eine Erhöhung der Glastemperatur bedeuten. Genauer gesagt, hängt die Fähigkeit zur Glasbildung vom Verhältnis der kovalenten Bindungsenergie zur Schmelztemperatur ab [Ell90]. Das führt dazu, dass im Experiment ein stärkerer Glasbildner als in der Simulation vorhanden ist, so dass bei gleichen Heiz- bzw. Kühlraten in der Simulation eine kleinere Glastemperatur als im Experiment erwartet werden kann.

Die Paarkorrelationsfunktionen der Proben zeigen mit den verbreiterten Peaks, deren Intensität mit der Entfernung abnimmt, den typischen Verlauf für eine ungeordnete Struktur [Was80]. Kristalline Anteile in der Probe können somit ausgeschlossen werden. Dass sich die Peaks für die höhere Temperatur ein wenig zu größeren Abständen verschieben, lässt sich mit thermischer Ausdehnung erklären. Insbesondere die Proben ohne Oberfläche bei $T=0 \mathrm{~K}$ zeigen sehr deutlich die Aufspaltung des zweiten Peaks, wie er aufgrund der vorhandenen Nahordnung für metallische Gläser typisch ist [Was80]. Dass sich die relative Höhe der Teilpeaks in diesem zweiten Hauptmaximum mit der $\mathrm{Zu}-$ sammensetzung ändert, lässt sich durch die unterschiedlichen Atomgrößen erklären. Je mehr von den größeren Ti Atomen enthalten ist desto größer ist nämlich der Teilpeak bei größerem Abstand. Dass die Aufspaltung bei erhöhter Temperatur und in Anwesenheit von Oberflächen undeutlicher wird, lässt sich zum einen durch eine Verschmierung der atomaren Abstände durch thermische Schwingungen erklären. Thermische Schwingungen tragen nämlich in Form des Debye-Waller-Faktors neben der statischen Unordnung in einer amorphen Struktur zur Verbreiterung der Peaks bei [Ell90]. Zum anderen sind 
die Gleichgewichtsabstände im Oberflächenbereich etwas anders als im Probeninneren, was auch eine Verschmierung der Peaks bewirken kann. Weiterhin sind die thermischen Schwingungsamplituden im Oberflächenbereich noch größer als im Probeninneren, so dass die Oberflächen das thermische Verschmieren noch weiter verstärken.

Die Proben zeigen also bei $T=0 \mathrm{~K}$ deutlich die Struktur metallischer Gläser und auch bei $T=450 \mathrm{~K}$ in Anwesenheit von Oberflächen bilden sich keine kristallinen Teilbereiche, so dass anders gesagt die Glasstruktur weiterhin erhalten ist.

\subsubsection{Untersuchung der atomaren Dynamik}

Die Auswertung der Diffusionsprofile in Abb. 6.5 zeigt sehr deutlich den Einfluss der Oberfläche auf die atomare Dynamik. Dabei bewirkt die Oberfläche keine deutliche Änderung der Zusammensetzung, die ebenfalls für eine Änderung des Diffusionsverhaltens verantwortlich sein könnte. Eine deutliche Änderung der Zusammensetzung könnte wiederum auch eine Änderung der Ordnung bewirken, da reines Kupfer bzw. Titan sicherlich eine deutlich stärkere Tendenz zur Kristallisation hätte. Deshalb passt dieses Ergebnis gut zu der im vorigen Kapitel diskutierten Tatsache, dass die Probe auch mit Oberflächen bei $T=450 \mathrm{~K}$ vollständig amorph ist.

Die Diffusionsprofile zeigen insbesondere auch, dass der Einfluss der Oberfläche auf wenige Ångström begrenzt ist. Bereits in [BT99; Böd99] wurde für das metallische Glas NiZr für die über die Elemente gemittelten Diffusionsprofile ein sehr ähnliches Verhalten gesehen. In diesen Arbeiten konnte auch gezeigt werden, dass die Werte der Diffusionskoeffizienten im Probeninneren mit denen von Bulkproben übereinstimmen. Diese Tatsache sollte auch hier gelten, da für den inneren Bereich $(|z|<20 \AA)$ kein Einfluss der Entfernung zur Oberfläche besteht. Die gute Übereinstimmung des Tiefenprofils mit der Fitfunktion Gl. (6.6) zeigt, dass die Ergebnisse sehr gut mit den aus allgemeinen thermodynamischen Betrachtungen im Rahmen des Landaumodells für Phasenübergänge resultierenden Erwartungen übereinstimmen. Dementsprechend scheint es sich hier um keinen materialspezifischen sondern um einen allgemeinen Oberflächeneffekt zu handeln. Experimentell ist ein solcher Oberflächeneffekt direkt nur schwierig zu untersuchen. Die erhöhte Beweglichkeit zeigt sich aber beispielsweise durch Einfügen zusätzlicher Grenzflächen in Form von Poren $\left[\mathrm{ASG}^{+}\right.$97].

In allen Bereichen der Probe zeigen die $\mathrm{Cu}$ Atome einen höheren Diffusionskoeffizienten als die Ti Atome unabhängig von der Anwesenheit von Oberflächen. Eine mögliche Erklärung dieses Verhaltens bezieht sich auf die unterschiedliche Atomgröße der Elemente. Im betrachteten System sind die $\mathrm{Cu}$ Atome die kleineren und die Ti Atome die größeren (Abstand nächster Nachbarn: $a_{C u}=2.56 \AA, a_{T i}=2.89 \AA$ ) [Kit93]. So zeigen die meisten metallischen Gläser zumindest für Fremddiffusion den Effekt, dass kleinere Atome für die Diffusion eine kleinere Aktivierungsenthalpie $H$ besitzen und das führt wiederum zu einem höheren Diffusionskoeffizienten $\left[\mathrm{FFM}^{+} 03\right]$. In dem zitierten Artikel wird erörtert, dass sich der Größeneffekt im Wesentlichen über die Aktivierungsenthalpie und weniger über den Faktor $D_{0}$ äußert $\left(D=D_{0} e^{-H /\left(k_{B} T\right)}\right)$. Als Erklärung für den Größeneffekt im Amorphen wird angeführt, dass die Abhängigkeit der Diffusion von der Atomgröße ähnliche Ursachen wie in einer kristallinen Struktur hat, weil die Nahord- 
nung ähnlich ist. Dort gibt es einen elastischen Beitrag der Matrix während der Diffusion. Dieser Beitrag führt zu einer erhöhten Aktivierungsenthalpie bei wachsender Größe des diffundierenden Atoms. Des Weiteren gibt es einen elektronischen Beitrag, der mit der chemischen Bindung zusammenhängt und in der Regel eine verminderte Aktivierungsenthalpie bei wachsender Valenz bewirkt $\left[\mathrm{FFM}^{+}\right.$03]. In vielen Bereichen des Periodensystems entspricht wachsende Valenz innerhalb einer Periode auch einer wachsenden Atomgröße und damit sinkt in diesem Fall die Aktivierungsenthalpie mit wachsender Atomgröße. Somit würden beide Effekte entgegengesetzte Wirkung haben. Im Falle der hier betrachteten Selbstdiffusion in einer binären Legierung sind die Eigenschaften des diffundierenden Atoms relativ zu denen in der umgebenden Matrix zu betrachten. Der elastische Effekt wäre dann eine mögliche Erklärung dafür, dass $\mathrm{Cu}$ Atome eine kleinere Aktivierungsenthalpie benötigen als die größeren Ti Atome. Bezüglich des elektronischen Effekts verhält es sich im betrachteten System so, dass $\mathrm{Cu}$ eine höhere Zahl an Valenzelektronen als Ti hat. Für die Übergangsmetalle der Periode, zu der beide Atomsorten gehören, beeinflussen sowohl die 3d als auch die 4s Elektronen die chemischen Bindungen und zählen somit zu den Valenzelektronen [Mor96]. Höhere Valenz bedeutet nach $\left[\mathrm{FFM}^{+} 03\right]$ eine kleinere Aktivierungsenthalpie, so dass dieser Effekt wiederum eine kleinere Aktivierungsenthalpie für $\mathrm{Cu}$ vorhersagt. Das Außergewöhnliche bezüglich des elektronischen Effekts ist hier, dass das Atom mit höherer Valenz die kleinere Atomgröße aufweist. Für die meisten metallischen Gläser ist der elastische Effekt der dominierende und der elektronische ist oft vernachlässigbar [FFM+03]. Im betrachteten System sprechen also alle Tatsachen dafür, dass $\mathrm{Cu}$ eine kleinere Aktivierungsenthalpie und damit verbunden einen höheren Diffusionskoeffizienten als Ti besitzt, so dass die Tendenzen der Modelle mit den Simulationsergebnissen übereinstimmen.

Weiterhin haben die Simulationen ergeben, dass sich, je nach dem Konzentrationsverhältnis von $\mathrm{Cu}$ und Ti Atomen, die Diffusionskoeffizienten beider Elemente ändern. Dabei werden die über die Probe gemittelten Werte für beide Elemente mit steigendem $\mathrm{Cu}$ Anteil kleiner (siehe Tab. 6.2). Die Beweglichkeit verringert sich also mit Zunahme des Anteils der Atomsorte, die innerhalb der Legierung die beweglichere ist. Eine Erklärung dafür könnte sein, dass bei einem großen Anteil der kleineren Atomsorte die Packungsdichte der kleineren Atome weiter erhöht ist. Wenn ein großer Anteil von großen Atomen eine schwer bewegliche Matrix bildet, können sich die kleinen Atome relativ leicht hindurchbewegen. Sind dagegen viele kleine Atome vorhanden, ist das nicht mehr möglich, weil sie dann selbst eine weniger leicht bewegliche Matrix bilden. Dass für ein binäres System, bei dem sich die Atomgrößen nur schwach unterscheiden $\left(a_{C u} / a_{T i}=2.56 / 2.89=0.89\right)$ eine Erhöhung des Anteils kleiner Atome zu einer verlangsamten Dynamik führt, konnte bereits im Rahmen von MD-Simulationen für ein Modell harter Kugeln gezeigt werden $\left[\mathrm{FGS}^{+}\right.$03]. Diese Übereinstimmung rechtfertigt die Argumentation über die Atomgröße, um Effekte in der Dynamik zu erklären. Es gibt auch experimentelle Untersuchungen von dünnen $\mathrm{CuTi}$ Schichten mit variabler Zusammensetzung, die einen Aufschluss über das diffusive Verhalten zulassen [Hül98; GHK98]. In diesen Arbeiten wurden insbesondere mechanische Spannungen während des Filmwachstums durch Kokondensation untersucht, die wiederum mit dem Diffusionsverhalten in Verbindung stehen. Beim Wachstum der amorphen CuTi Schichten zeigt sich, 
dass während der ersten Atomlagen (einige nm) Zugspannungen herrschen. Diesem Bereich folgt ein Bereich, in dem Druckspannungen vorherrschen, während die Oberflächen eine relativ glatte Struktur aufweisen. Daran schließt sich je nach Zusammensetzung nach einigen hundert Nanometern ein Bereich mit Zugspannungen an, in dem die Oberflächenstruktur hügel- bzw. säulenartig ist. Der Zusammenhang zwischen der Spannungsentwicklung und der Oberflächenstruktur bzw. den Wachstumsmechanismen wird in [May00] ausführlich untersucht und diskutiert. Dabei ergibt sich, dass der Druckspannungsbereich mit relativ glatten Oberflächen und anwachsender Strukturgröße einhergeht. Daran schließt sich eine Sättigung der Strukturgröße an und es treten Zugspannungen auf, die durch die Koaleszenz von Hügeln - die im Druckspannungsbereich gewachsen sind - bedingt sind. Ist die Beweglichkeit kleiner, würde man erwarten, dass sich nur kleinere Strukturen bilden und demzufolge die Sättigung der Strukturgröße und der Einsatz von Zugspannungen bereits bei kleineren Schichtdicken stattfindet. Die $\mathrm{Cu}_{40} \mathrm{Ti}_{60}$ Schichten zeigen einen ausgedehnten Druckspannungsbereich, während die beiden anderen Zusammensetzungen von den anfänglich starken Zugspannungen zu sehr schwachen Zugspannungen, die sich dann im Fall von $\mathrm{Cu}_{50} \mathrm{Ti}_{50}$ bei dicken Schichten nochmals zu stärkeren Zugspannungen entwickeln, übergehen [Hü198]. Ein ausgedehnter Druckspannungsbereich, der mit einer glatten Oberflächenstruktur verbunden ist, kann nur existieren, wenn die Diffusivität der Oberflächenatome groß genug ist, um die Oberfläche während des Wachstums zu glätten, bevor die nächsten Atome auftreffen. Diese Interpretation der gemessenen Spannungen liefert also für $\mathrm{Cu}_{40} \mathrm{Ti}_{60}$ einen höheren Diffusionskoeffizienten als für $\mathrm{Cu}_{50} \mathrm{Ti}_{50}$ und $\mathrm{Cu}_{60} \mathrm{Ti}_{40}$. Betrachtet man die Spannungsentwicklung im Anfangsstadium des Schichtwachstums, so wird deutlich, dass sich für $\mathrm{Cu}_{60} \mathrm{Ti}_{40}$ die Zugspannungen bis zu etwas größeren Schichtdicken fortsetzen, als es bei $\mathrm{Cu}_{50} \mathrm{Ti}_{50} \mathrm{zu}$ beobachten ist. Bei den $\mathrm{Cu}_{40} \mathrm{Ti}_{60}$ Schichten findet der Übergang bei noch geringeren Schichtdicken als bei $\mathrm{Cu}_{50} \mathrm{Ti}_{50}$ statt. Diese anfänglichen Zugspannungen sind wahrscheinlich durch ein anfängliches Inselwachstum und die dann stattfindende Koaleszenz dieser Inseln bedingt und halten daher an, bis sich eine geschlossene glatte Schicht gebildet hat [May00]. Bei höherer Beweglichkeit sollte dementsprechend die Zugspannung schon bei kleineren Schichtdicken abnehmen. Das spricht wiederum für eine abnehmende Beweglichkeit von $\mathrm{Cu}_{40} \mathrm{Ti}_{60}$ über $\mathrm{Cu}_{50} \mathrm{Ti}_{50}$ zu $\mathrm{Cu}_{60} \mathrm{Ti}_{40}$, so dass insgesamt bei den drei betrachteten Zusammensetzungen der Trend aus den experimentellen Spannungsmessungen mit den hier in der Simulation gefundenen Daten in Einklang gebracht werden kann.

Die Zunahme des Diffusionskoeffizienten im Oberflächenbereich gegenüber dem Probeninneren belegt den Einfluss der Oberfläche auf eine veränderte Dynamik. Allerdings ist dadurch noch nicht geklärt, ob und wie sich die Mechanismen der Diffusion ändern. Über diese Fragestellung geben die Verteilungen der Verschiebungsweiten Aufschluss. Die Unterschiede in Abb. 6.6 zwischen den Atomen im Probeninneren und im Oberflächenbereich deuten für beide Elemente einen unterschiedlichen Diffusionsmechanismus in den verschiedenen Probenbereichen an, da im Inneren die Gaußfunktion die Simulationsdaten relativ gut beschreiben kann, während das im Oberflächenbereich nicht möglich ist. Die Gaußfunktion beschreibt den Verlauf, der bei einem kollektiven Mechanismus erwartet wird. Insofern zeigen die Ergebnisse, dass im Probeninneren im 
Wesentlichen kooperative Prozesse ablaufen, weil im Bereich atomarer Abstände keine gegenüber der Umgebung erhöhte Verschiebungswahrscheinlichkeit erkennbar ist. Als kooperative Effekte können Umordnungsprozesse größerer Atomgruppen sowie auch thermische Schwingungen beitragen [Wah91]. Da auch im Inneren atomare Bewegungen von $2 \AA$ vorhanden sind, lässt sich der Peak in der Verteilung sicherlich nicht nur durch thermische Schwingungen erklären. Insofern muss sich der Hauptpeak aus thermischen Schwingungen und kleinen lokalen Umordnungen von Atomgruppen ergeben. Im Glas ist es allerdings nicht eindeutig möglich, thermische Schwingungen und Änderungen der Gleichgewichtsposition anhand der Größe der Verschiebung oder auch anhand einer Zeitskala scharf voneinander zu trennen, da sich im Gegensatz zum Kristall im Glas Umordnungen auf sehr breit verteilten Zeit- und Längenskalen abspielen können [Tei05]. Für beide Elemente wird beim Vergleich von Oberflächenbereich und Probeninnerem klar, dass die Form der Verteilung der Verschiebungsweiten unterschiedlich ist, weil an der Oberfläche der Anteil weiter Sprünge deutlich größer ist als es der Gaußverteilung nach Gleichung (6.8) entspricht. Insbesondere das für $\mathrm{Cu}$ Atome auftretende zweite Maximum im Oberflächenbereich, das seine Position ziemlich genau beim Nachbarabstand von $\mathrm{Cu}$ Atomen hat $\left(a_{C u}=2.56 \AA\right.$ [Kit93] $)$, zeigt die Änderung in der atomaren Dynamik. Die Position deutet darauf hin, dass sich eine signifikante Anzahl von $\mathrm{Cu}$ Atomen um den $\mathrm{Cu}-\mathrm{Cu}$ Nachbarabstand verschiebt. Diese Tatsache spricht wiederum dafür, dass sich diese Atome in Form von Einzelsprüngen bewegen, da in der Nahordnung die Entfernung der Plätze noch einigermaßen genau definiert ist. Möglich wäre auch eine Bewegung von Ketten, bei der sich jedes Atom um den Nachbarabstand verschiebt. Während solche Kettenbewegungen in Bulkproben auftreten [OS99; Tei01], sind an Oberflächen auch echte Einzelsprünge möglich. Bei anderen Bewegungen größerer Atomgruppen gäbe es dagegen keine Begründung für eine solche ausgezeichnete Verschiebungslänge. Dass dieser zweite Peak relativ breit ist, stimmt mit der Erwartung überein, weil im Glas trotz der existierenden Nahordnung die Abstände nächster Nachbarn um einen Mittelwert herum verteilt sind, wie in den Paarkorrelationsfunktionen (vgl. Abb. 6.2) erkennbar ist. Das Hauptmaximum bei kleineren Abständen existiert weiterhin, weil nicht alle Oberflächenatome notwendigerweise Einzelsprünge durchführen. Die Einzelsprünge sind den weiterhin existierenden kooperativen Prozessen also überlagert. Außerdem ist die Verteilung der Atome aus der äußeren $5 \AA$ dicken Schicht dargestellt und in dieser Schicht befinden sich auch noch viele Atome, die ausgehend von ihren Bindungsverhältnissen nicht als Oberflächenatome im engeren Sinn bezeichnet werden können und daher in der Regel nur an kooperativen Prozessen beteiligt sind. Allerdings ist auch für sie die Beweglichkeit gegenüber dem Probeninneren bereits erhöht (siehe Abb. 6.5). Als Illustration dieser Ergebnisse zeigt auch das Verhalten einzelner ausgewählter Atome, dass es tatsächlich solche $\mathrm{Cu}$ Atome im Oberflächenbereich gibt, die sich durch Einzelsprünge fortbewegen (siehe Kap. 6.1.5). Grund für diese unterschiedliche Dynamik im Inneren und an der Oberfläche ist sicherlich die zusätzliche Bewegungsfreiheit an der Oberfläche. Die Atome auf der Oberfläche können sich aufgrund der Bindungsverhältnisse in ihrer Umgebung bewegen, ohne das sich andere Atome notwendigerweise mitbewegen müssen. Im Probeninneren ist diese Möglichkeit dagegen nicht vorhanden. Das Auftreten eines zweiten Maximums zeigt eine zusätzliche Dynamik an, indem Sprünge zu Nachbarplätzen 
zusätzlich zu kleineren Bewegungen stattfinden.

Beim Vergleich der beiden Elemente wird klar, dass die $\mathrm{Cu}$ Atome eine wesentlich deutlichere Tendenz zu Einzelsprüngen zeigen als die Ti Atome, für die sich kein zweites Maximum sondern lediglich eine Schulter in der Verteilung ausbildet. Ein Erklärungsansatz bezieht sich, wie schon bei der Begründung der erhöhten Diffusionskoeffizienten der $\mathrm{Cu}$ Atome, auf die unterschiedlichen Atomgrößen. Auch im Oberflächenbereich müssen die Atome für einen Sprung Energiebarrieren, die sich durch die Anordnung benachbarter Atome ergeben, überwinden. Solche Energiebarrieren für das Durchdringen der Matrix aus umgebenden Atomen sind aus geometrischen Gründen kleiner für die kleinere Atomsorte, denn sie benötigen ein kleineres Aktivierungsvolumen. Dass die kleinere Atomsorte eher die Tendenz für ein zweites Maximum zeigt, gilt auch für Bulkproben metallischer Gläser im Temperaturbereich der unterkühlten Schmelze [GKS99]. Aufgrund der Oberfläche reichen bei den in diesem Kapitel gezeigten Ergebnissen bereits kleinere Temperaturen deutlich unterhalb der Bulk-Glastemperatur aus, um ähnliche Effekte zu sehen.

Die Betrachtungen bezüglich der Energieverteilung haben gezeigt, dass $\mathrm{Cu}$ Atome an der Oberfläche im Vergleich zum Probeninneren eine erhöhte (also vom Betrag her kleinere) potentielle Energie aufweisen, während dies bei den Ti Atomen nicht der Fall ist. Dieser Unterschied zwischen beiden Elementen passt qualitativ zu dem Ergebnis, dass für $\mathrm{Cu}$ Atome Einzelsprünge an der Oberfläche eine deutlich höhere Wahrscheinlichkeit haben als es für Ti Atome der Fall ist. Im Vergleich zum Probeninneren sind an der Oberfläche die $\mathrm{Cu}$ Atome gegenüber den Ti Atomen bezüglich der potentiellen Energie auf einem höheren Niveau, so dass wegen einer daher gesunkenen Aktivierungsenthalpie die erhöhte Mobilität zu erwarten ist. Diese erhöhte $\mathrm{Cu}$ Mobilität kann nun wiederum auch eine erhöhte Beweglichkeit der Ti Atome induzieren, weil sich die lokale Umgebung der $\mathrm{Ti}$ Atome an den Orten, wo $\mathrm{Cu}$ Einzelsprünge vorkommen, ändert. Wegen dieser Abhängigkeit sind die Ti Atome bei ihrer Bewegung weiterhin einem kooperativen Prozess unterworfen.

Es bleibt nun noch die Frage, warum sich $\mathrm{Cu}$ und Ti Atome bezüglich der Verteilung ihrer potentiellen Energien unterschiedlich verhalten. Generell würde man erwarten, dass Oberflächenatome weniger stark gebunden sind als Atome aus dem Probeninneren, denn durch diesen Unterschied zwischen Oberfläche und Probeninnerem ergibt sich eine von null verschiedene Oberflächenenergie, die sowohl für reines $\mathrm{Cu}$ als auch für reines Ti experimentell mit den Werten $\gamma_{C u}=1.77 \mathrm{~J} / \mathrm{m}^{2}$ und $\gamma_{T i}=1.92 \mathrm{~J} / \mathrm{m}^{2}$ beobachtet wird [TM77]. So gesehen entsprechen die $\mathrm{Cu}$ Atome den Erwartungen und die Ti Atome nicht.

Eine Erklärungsmöglichkeit für das unterschiedliche Verhalten ist, dass die oberflächennahen $\mathrm{Ti}$ Atome stets von $\mathrm{Cu}$ Atomen umgeben sind, die dann die eigentlichen Oberflächenatome wären. Nach einem Blick auf die Oberfläche (siehe Abb. 6.12) kann man diesen Erklärungsansatz allerdings ausschließen, weil beide Atomsorten zu gleichen Anteilen an der Oberfläche vorhanden sind. Dass die Ti Atome im Wesentlichen an der Oberfläche keine erhöhte potentielle Energie gegenüber dem Probeninneren aufweisen, ist zunächst überraschend, weil aufgrund der Geometrie weniger Nachbarn vorhanden sind. Es könnte sich hier also um eine Unzulänglichkeit im Ti Anteil des verwendeten Potentials handeln. Allerdings gibt es bei Mehrkörperpotentialen der hier verwendeten Art einen 


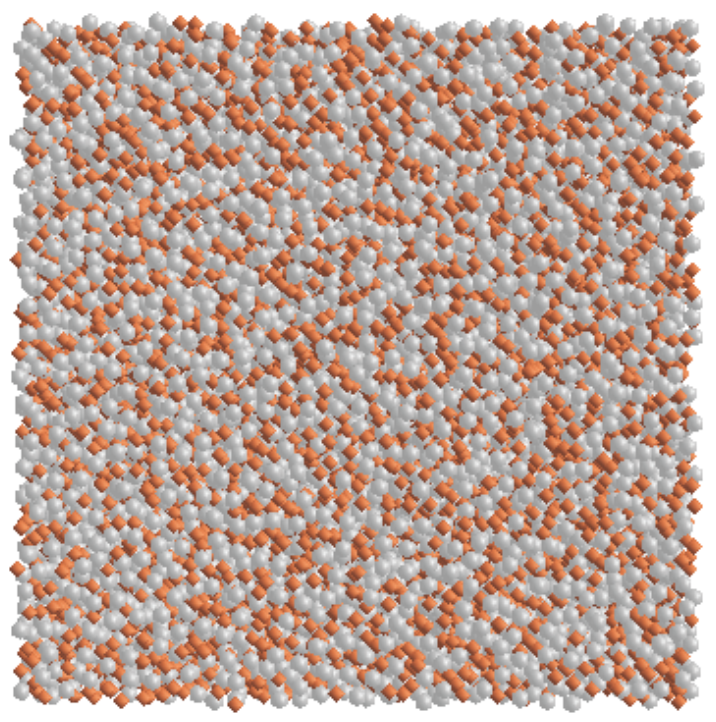

Abbildung 6.12: Oberfläche der bezüglich der Energieverteilung analysierten $\mathrm{Cu}_{50} \mathrm{Ti}_{50}$ Probe; braun: Cu Atome, grau: Ti Atome

Effekt, der erklären kann, dass die Bindung eines Oberflächenatoms nicht zwangsläufig schwächer ist als im Probeninneren: Oberflächenatome haben zwar weniger Nachbarn, aber dafür können die einzelnen Bindungen, die die Oberflächenatome ausbilden, stärker sein. Im Gegensatz zu einem Paarpotential ist bei einem Mehrkörperpotential, wie es hier verwendet wird, die Bindungsstärke zwischen zwei Atomen nämlich von der lokalen Umgebung abhängig. Diese Eigenschaft ist auch dafür verantwortlich, dass in vielen mit einem EAM Potential modellierten Übergangsmetallen wie auch $\mathrm{Cu}$, die obere Atomlage im Durchschnitt einen kleineren Abstand zur benachbarten Lage hat als es im Probeninneren zu beobachten ist [FBD86]. Im Oberflächenbereich ist nämlich die Elektronendichte gegenüber dem Probeninneren erhöht und das führt zu stärkeren Bindungen. Dieser Effekt ist auch experimentell beobachtet [LWRW84], was für eine gute Näherung des EAM Modells an die Realität auch für Oberflächen spricht.

Eine weitere Erklärungsmöglichkeit ist, dass sich die erwartete Energieerhöhung an der Oberfläche durch eine verstärkte Energieerhöhung der $\mathrm{Cu}$ Atome bei nahezu unverändertem Energieniveau der Ti Atome äußert. Dieser Erklärungsansatz erscheint sinnvoll, wenn man die Oberflächenenergien betrachtet. In Kap. 7 werden diese für CuTi 
bei $\mathrm{T}=10 \mathrm{~K}$ und $\mathrm{T}=300 \mathrm{~K}$ berechnet mit dem identischen Ergebnis $\gamma=1.14 \mathrm{~J} / \mathrm{m}^{2}$. Für das reine $\mathrm{Cu}$ System ergibt sich bei kristallinen Oberflächen je nach ihrer Orientierung im EAM Potential nach $\left[\right.$ FBD86] $\gamma(111)=1.17 \mathrm{~J} / \mathrm{m}^{2}, \gamma(100)=1.28 \mathrm{~J} / \mathrm{m}^{2}$ und $\gamma(110)=1.40 \mathrm{~J} / \mathrm{m}^{2}$. Insofern sind die Oberflächenenergien für $\mathrm{CuTi}$ und $\mathrm{Cu}$ sehr ähnlich. Da im CuTi die Ti Atome aber im Inneren und an der Oberfläche annähernd gleiche potentielle Energie aufweisen, heißt das, dass die $\mathrm{Cu}$ Atome in der Legierung ihre Energie zwischen Oberfläche und Probeninnerem umso mehr ändern, da sich die Zusammensetzung zwischen beiden Bereichen der Probe nicht wesentlich unterscheidet. Die Abhängigkeit der Bindungseigenschaften von der lokalen Umgebung bei Mehrkörperpotentialen lässt insbesondere auch zu, dass sich die Energien der beiden Komponenten relativ zueinander unterschiedlich verhalten, da die Elektronendichte im Oberflächenbereich gegenüber dem Probeninneren erhöht ist.

Dass bei den variierenden Zusammensetzungen die Energien beider Atomsorten mit wachsendem $\mathrm{Cu}$ Anteil ansteigen, passt zu dem Resultat, dass reines $\mathrm{Cu}$ in einer EAM Modellierung eine höhere potentielle Energie aufweist als reines Ti [BSS96]. Dass in der Legierung dagegen die $\mathrm{Cu}$ Atome stets eine kleinere Energie als die Ti Atome aufweisen, muss durch die veränderte Nachbarschaft der einzelnen Atome im Vergleich zum reinen Material begründet sein. Den Ergebnissen entsprechend ist sowohl für die $\mathrm{Cu}$ als auch für die Ti Atome eine Bindung zu einem Ti Atom energetisch günstiger als zu einem $\mathrm{Cu}$ Atom. So lässt sich begründen, dass $\mathrm{Cu}$ Atome in der Legierung eine kleinere und Ti Atome eine größere potentielle Energie aufweisen als im reinen Material. Die Abbildung 6.12 zeigt, dass insgesamt die Nachbarschaft verschiedener Atomsorten bevorzugt wird, da es zu keiner Phasenseparation kommt.

\subsection{Amorphes Si}

In diesem Abschnitt sollen Ergebnisse zu amorphem Silizium vorgestellt werden. Dabei sind insbesondere Unterschiede und Gemeinsamkeiten zwischen diesem starken Glas mit kovalenten Bindungsanteilen und dem eher fragilen metallischen Glas $\mathrm{CuTi}$, das in Kap. 6.1 besprochen wurde, von Interesse.

Die Analysemethoden und zum Teil die grundlegenden Erwartungen, wie sie in Kap. 6.1 beschrieben sind, gelten hier ebenso und werden daher nicht wiederholt.

\subsubsection{Herstellung, Struktur und Glasübergang}

\subsubsection{Thermodynamische Größen und Glasübergang}

Die Si Proben werden wie in Abschnitt 3.4 beschrieben hergestellt. Insbesondere wird der Druck während der Herstellung auf $p=0$ mbar geregelt, so dass Enthalpie und Energie im System identisch sind. Während des Abkühlens aus der Schmelze werden das Volumen und die Energie der Probe aufgenommen, so dass sich beide thermodynamischen Größen als Funktion der Probentemperatur darstellen lassen. Beide Größen werden in Abb. 6.13 für den Kühlprozess im Bereich des möglichen Glasübergangs gezeigt. In der 

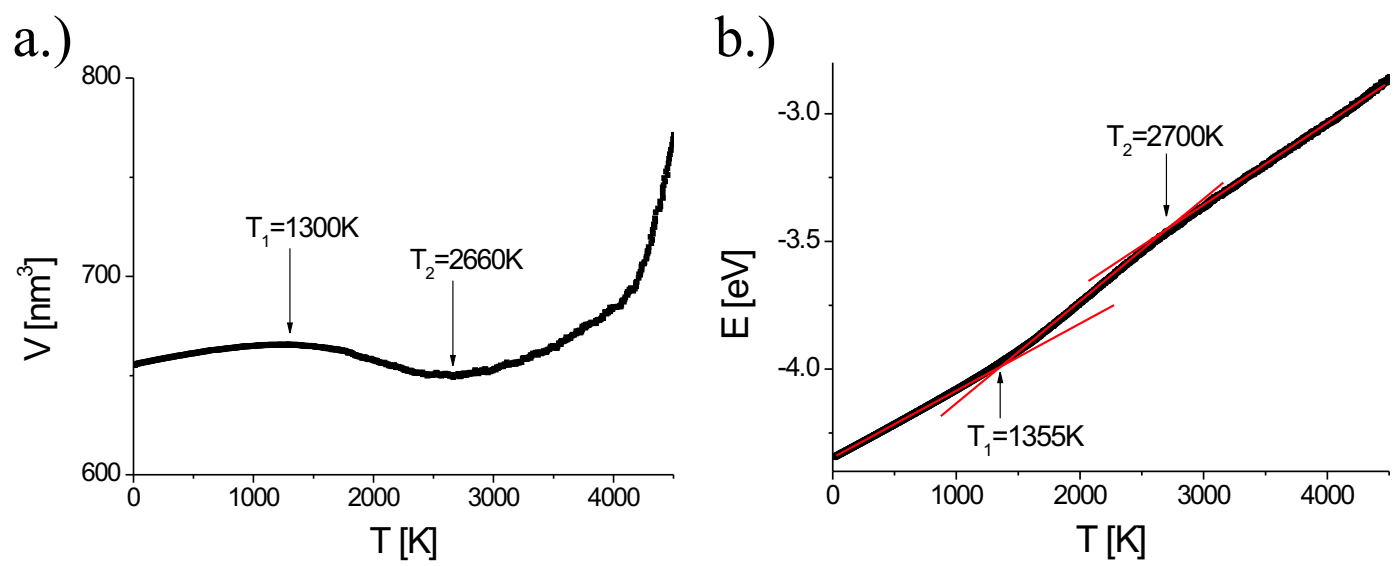

Abbildung 6.13: a.) Volumen und b.) Energie jeweils in Abhängigkeit der Temperatur beim Abkühlen (mit linearen Fits für die Energie) (Rate: $\dot{T}=-50 \mathrm{~K} / \mathrm{ps}$ ).

Auftragung des Volumens ergibt sich ein Maximum bei $T_{1}=1300 \mathrm{~K}$ und eine Minimum bei $T_{2}=2660 \mathrm{~K}$. Bei sehr ähnlichen Temperaturwerten, nämlich bei $T_{1}=1355 \mathrm{~K}$ bzw. $T_{2}=2700 \mathrm{~K}$, zeigt die Energie als Funktion der Temperatur jeweils eine markante Änderung der Steigung, also einen verschmierten Knick. Insofern sind diese beiden Temperaturbereiche und das dazwischenliegende Intervall Bereiche eines thermodynamischen Übergangs im System. Bemerkenswert ist an dieser Stelle zunächst der sehr unterschiedliche Verlauf des Volumens bzw. der Energie als Funktion der Temperatur. Dabei ist zu beachten, dass die beobachteten Knicke in der Auftragung der Energie verschiedene Richtungen haben. Der bei der tieferen Temperatur ist ein Knick zu größerer Steigung, während es bei der höheren Temperatur umgekehrt ist. Zwischen den zwei Temperaturbereichen ist die Steigung also am größten. Gerade in diesem Temperaturintervall zeigt die Auftragung des Volumens als Funktion der Temperatur einen negativen thermischen Ausdehnungskoeffizienten. Außerhalb dieses Intervalls ist der Ausdehnungskoeffizient dagegen positiv. Somit lässt sich bisher sagen, dass es nicht eindeutig nur eine Temperatur gibt, bei der ein Übergang stattfindet und die folglich als Glastemperatur bezeichnet werden soll (siehe Diskussion in Kap. 6.4.1).

\subsubsection{Strukturanalyse der Si Proben}

Um weitere Erkenntnisse über die Vorgänge in den Si Proben zu gewinnen, soll ihre Struktur mit Hilfe der Paarkorrelationsfunktion (zur Definition siehe Kap. 6.1.1.2) analysiert werden. Diese ist in Abb. 6.14 für Proben bei verschiedenen Temperaturen und Auslagerungszeiten dargestellt. Dabei ist die Abfolge so, dass die Paarkorrelation zuerst bei $T=10 \mathrm{~K}$ direkt nach dem Herstellungsprozess, also dem Abschrecken aus der Schmelze, berechnet wird. Danach wird die Probe mit einer Rate von $20 \mathrm{~K} / \mathrm{ps}$ auf $T=1400 \mathrm{~K}$ geheizt, um dann wiederum die Paarkorrelationsfunktion zu berechnen. Bei dieser Temperatur wird die Probe für eine Zeit von weiteren 4 ns ausgelagert, um danach erneut die Paarkorrelation auszuwerten. Im Anschluss daran wird die Probe mit 


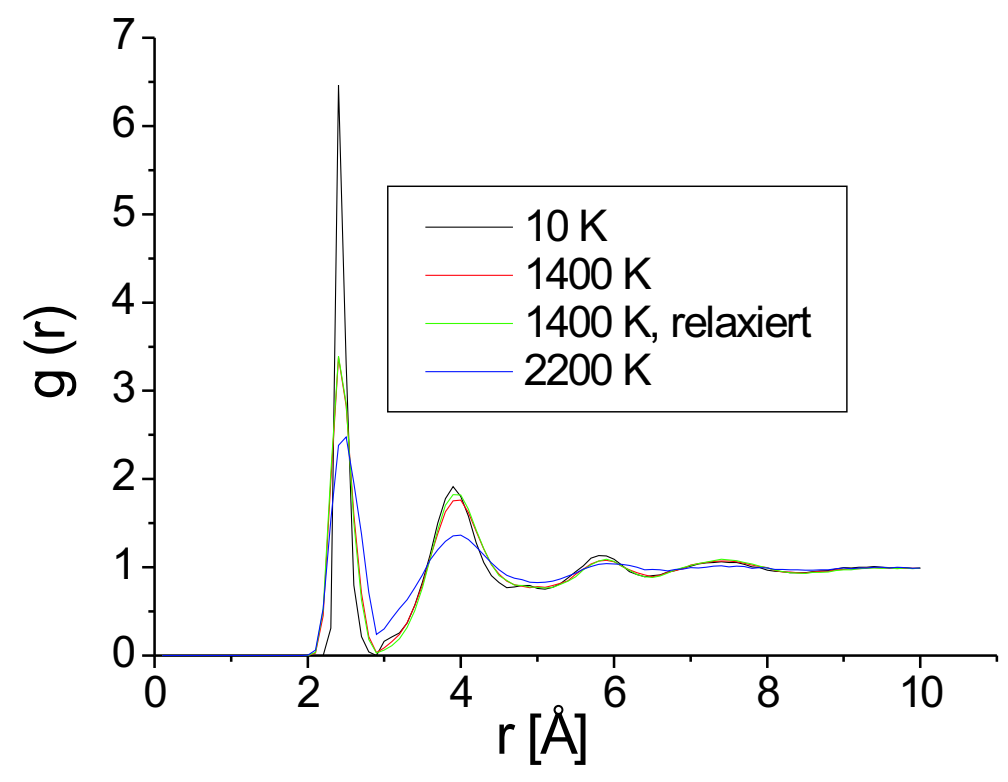

Abbildung 6.14: Paarkorrelationsfunktion der Si Bulk-Proben bei $T=0 \mathrm{~K}, T=1400 \mathrm{~K}$, $T=1400 \mathrm{~K}$ mit zusätzlicher Relaxation und bei $T=2200 \mathrm{~K}$.

gleicher Rate auf $T=2200 \mathrm{~K}$ geheizt und eine erneute Berechnung der Paarkorrelation findet statt. Deutlich erkennbar ist hier, dass mit steigender Temperatur die Intensität im ersten Maximum abnimmt und zugleich die Breite dieses Peaks zunimmt. Diese Tendenz trifft in abgeschwächter Form auch auf das zweite und dritte Maximum zu. Bei der Intensität im Bereich der Minima fällt auf, dass die Probe bei $T=2200 \mathrm{~K}$ deutlich größere Werte als die anderen, die untereinander diesbezüglich sehr ähnlich sind, zeigt. Die Probe bei $T=10 \mathrm{~K}$ zeigt als einzige ein kleines Nebenmaximum im Bereichen eines Hauptminimums. Alle Proben haben zwischen $r=9 \AA$ und $r=10 \AA$ bereits einen nahezu konstanten Wert erreicht und außerdem ist allen Proben gemein, dass für zunehmenden Abstand die Peaks an Intensität verlieren. Nachdem in Abb. 6.14 somit der Einfluss von Temperatur und Relaxation auf die Struktur dargestellt ist, soll nun der Einfluss von Oberflächen dargestellt werden. In Abb. 6.15 ist daher die Paarkorrelationsfunktion der bei $T=1400 \mathrm{~K}$ relaxierten Probe aus Abb. 6.14 zusammen mit der Paarkorrelationsfunktion der aus dieser Probe durch Öffnen der Oberflächen und weitere 300 ps Relaxation entstandenen Probe aufgetragen. Dabei ist zu beachten, dass in beiden Fälle alle Atome berücksichtigt werden und somit auch die Probe mit den Oberflächen zum größten Teil aus Bulk-Atomen besteht. Es ist kein wesentlicher Unterschied zwischen beiden Kurven vorhanden. 


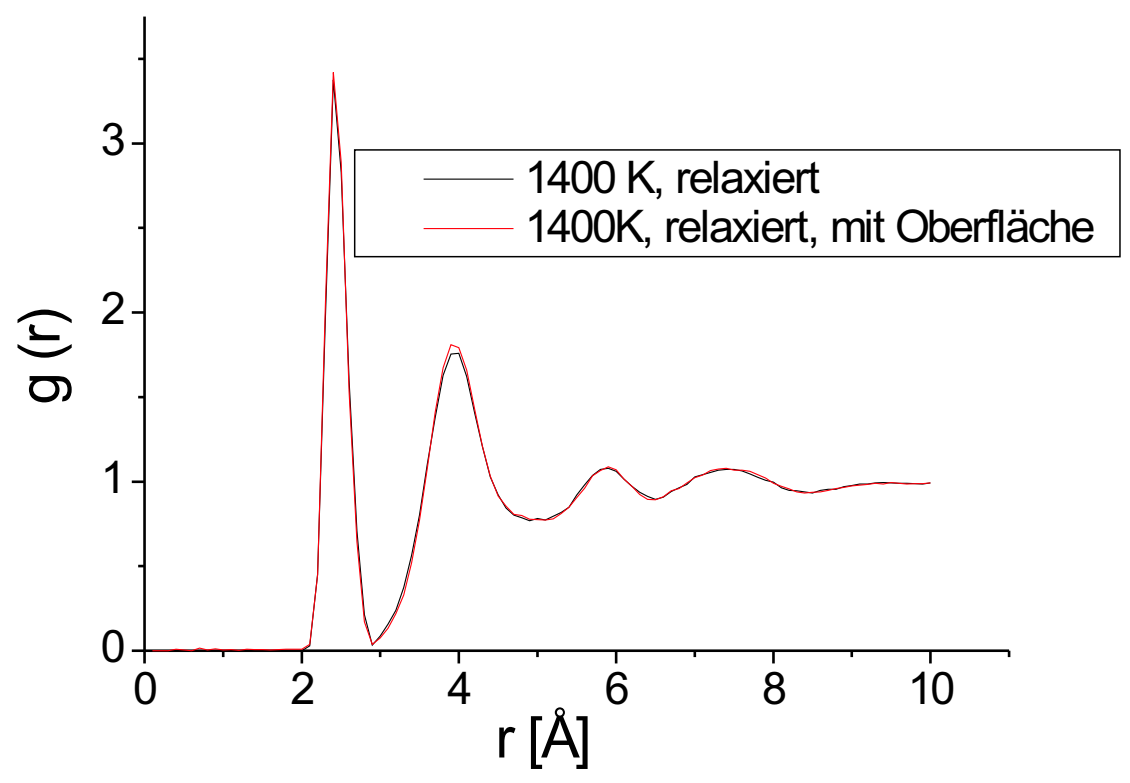

Abbildung 6.15: Paarkorrelationsfunktion der relaxierten Si Proben bei $T=1400 \mathrm{~K}$ als Bulk-Probe und mit zwei Oberflächen, die für einen Zeitraum von 300 ps relaxiert sind.

\subsubsection{Dynamik der Si Schichten}

Wie bereits für das metallische Glas geschehen, soll auch für das amorphe Si der Einfluss der Oberfläche anhand von tiefenabhängigen Diffusionsprofilen und der Verteilung von Verschiebungsweiten untersucht werden. Dafür werden zunächst die bei $T=1400 \mathrm{~K}$ relaxierten Proben analysiert. Sie sind bei $T=1400 \mathrm{~K}$ vor dem Erzeugen der Oberflächen für $4 \mathrm{~ns}$ und danach für weitere 300 ps relaxiert. Die Proben bei $T=1000 \mathrm{~K}$ bzw. $T=1700 \mathrm{~K}$ sind aus den bei $1400 \mathrm{~K}$ relaxierten entstanden und nach Öffnen der Oberflächen ebenfalls für weitere 300 ps relaxiert. Die Diffusion wird bei allen Temperaturen gemäß Gleichung (6.2) aus den Bewegungen in einem Zeitraum von 500 ps berechnet. Für die Intervallgröße in z-Richtung wird auch hier $\Delta z=1 \AA$ verwendet. Im Oberflächenbereich sind nur die Werte für die z-Intervalle aufgetragen, in denen mehr als 10 Atome liegen, weil sonst die statistischen Schwankungen für den Wert des Diffusionskoeffizienten in diesem Bereich zu groß wären. Im Inneren der Probe befinden sich durchschnittlich ungefähr 370 Atome in jedem Intervall. In Abb. 6.16 ist ein Anstieg des Diffusionskoeffizienten im Oberflächenbereich gegenüber dem Probeninneren für alle Temperaturen klar erkennbar. Der Diffusionskoeffizient ist in den äußeren Intervallen gegenüber dem Probeninneren im Falle der Schichten bei $T=1000 \mathrm{~K}$ und $T=1400 \mathrm{~K}$ um fast eine Größenordnung erhöht. Bei $T=1700 \mathrm{~K}$ ist die Erhöhung zur Oberfläche hin etwas geringer. Der Diffusionskoeffizient nimmt mit steigender Temperatur deutlich zu. Außerdem wird der Bereich, der durch die Oberfläche beeinflusst wird, mit steigender 


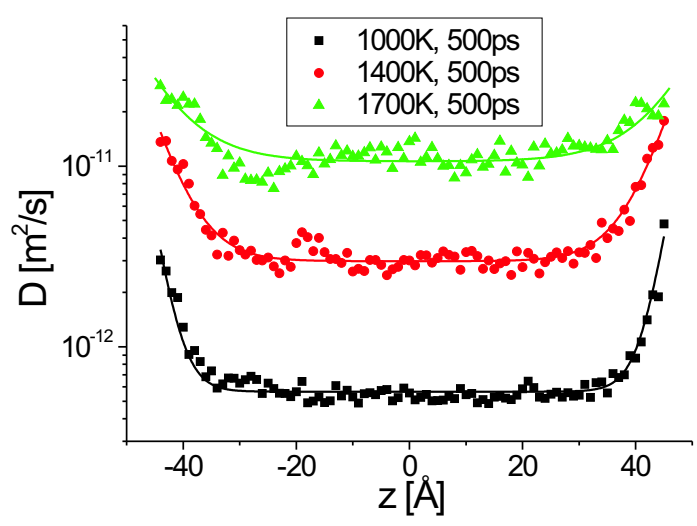

Abbildung 6.16: Tiefenprofil des Diffusionskoeffizienten der relaxierten Si Probe bei $T=1000 \mathrm{~K}, T=1400 \mathrm{~K}$ und $T=1700 \mathrm{~K}$ jeweils in einem Zeitintervall von $\Delta t=500 \mathrm{ps}$ mit zwei Oberflächen in z-Richtung.

\begin{tabular}{|c|c|c|c|}
\hline & $1000 \mathrm{~K}$ & $1400 \mathrm{~K}$ & $1700 \mathrm{~K}$ \\
\hline$D_{\infty}\left[10^{-12} \cdot \mathrm{m}^{2} / \mathrm{s}\right]$ & $0.565 \pm 0.019$ & $2.98 \pm 0.08$ & $10.6 \pm 0.3$ \\
\hline$z_{0}[\AA]$ & $2.29 \pm 0.15$ & $4.35 \pm 0.20$ & $6.42 \pm 0.83$ \\
\hline
\end{tabular}

Tabelle 6.4: Fitparameter $D_{\infty}$ und $z_{0}$ gemäß Gleichung (6.6) zu Abb. 6.16 bei verschiedenen Temperaturen.

Temperatur größer. Die Daten aus der Simulation lassen sich durch den in Abb. 6.16 eingetragenen Fit gemäß Gleichung (6.6) recht gut beschreiben. Die physikalisch interessanten Fitparameter aus Gleichung (6.6), die zum einen den Diffusionskoeffizienten im Probeninneren beschreiben und zum anderen ein Maß für die charakteristische Breite des exponentiellen Anstiegs im Oberflächenbereich sind, sind in Tab. 6.4 zusammengefasst. Sie bestätigen die oben beschriebenen Temperaturabhängigkeiten für die tiefenabhängigen Diffusionskoeffizienten und die Größe des Einzugsbereichs der Oberfläche. Auffällig ist, dass bei $T=1400 \mathrm{~K}$ und noch mehr bei $T=1700 \mathrm{~K}$ die Streuung der Diffusionskoeffizienten der einzelnen Intervalle größer ist als bei $T=1000 \mathrm{~K}$. Bei $T=1400 \mathrm{~K}$ gibt es eine deutliche Abweichung von der Fitkurve im Bereich $-20 \AA<z<-15 \AA$ zu großen Werten. Bei $T=1700 \mathrm{~K}$ gibt es mehrere Bereiche mit deutlichen Schwankungen.

Die Histogramme der Verschiebungsweiten sind für die drei bisher behandelten Temperaturen jeweils für den Oberflächenbereich $(|z|>40 \AA)$ und das Probeninnere $(|z|<20 \AA)$ in Abb. 6.17 dargestellt. Die Achsen der drei Graphen sind unterschiedlich skaliert, um die entscheidenden Charakteristika möglichst gut sichtbar zu machen. Bei $T=1000 \mathrm{~K}$ passt für das Probeninnere der Fit gemäß der Gaußkurve aus Gl. (6.8) nahezu perfekt. Im Oberflächenbereich bildet sich im Gegensatz dazu auf der rechten Flanke des Maximums eine kleine Schulter heraus, die aber nur sehr schwach ausgeprägt ist. Daher gibt auch für den Oberflächenbereich die Gaußkurve den Verlauf recht gut wieder. Bei $T=1400 \mathrm{~K}$ passt die Gaußkurve im Probeninneren immer noch sehr gut zu den Daten, 
a.)

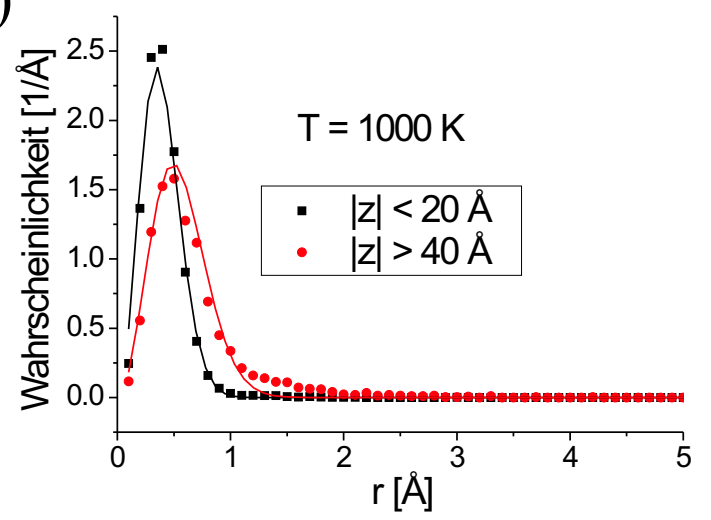

b.)

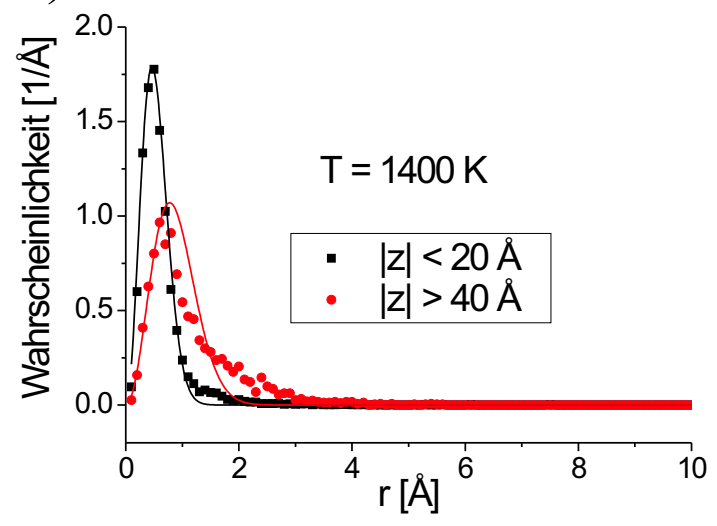

c.)

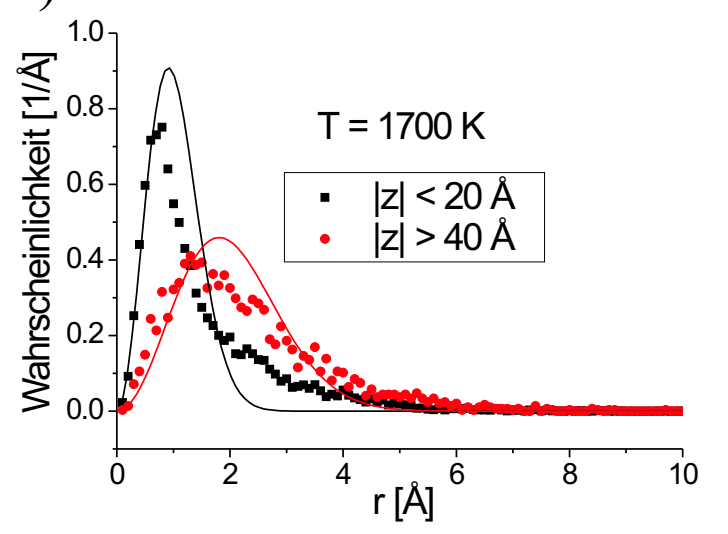

Abbildung 6.17: Histogramme der Verschiebungsweiten nach jeweils $\Delta t=500$ ps für dieselben Proben wie in Abb. 6.16 bei a.) $T=1000 \mathrm{~K}$, b.) $T=1400 \mathrm{~K}$, c.) $T=1700 \mathrm{~K}$. Die Linien entsprechen einem Fit nach Gleichung (6.8).

wenngleich sich nun auch hier auf der rechten Seite des Hauptpeaks eine kleine Schulter bildet. Im Oberflächenbereich ist diese Schulter dagegen bereits deutlich verstärkt. Hier weicht nun außerdem auch insgesamt die angepasste Gaußkurve deutlich von den Daten ab. Das Gewicht des Beitrags aus dem Bereich der Schulter ist hier also signifikant. Bei $T=1700 \mathrm{~K}$ zeigen sowohl die Daten im Probeninneren als auch im Oberflächenbereich deutliche Abweichungen von den angepassten Gaußkurven. Das Maximum der Daten liegt hier immer bei kleineren Werten als das der Gaußkurve. Beim Vergleich der Kurvenverläufe als Funktion der Temperatur fällt auf, dass sich alle Peaks mit steigender Temperatur verbreitern und zu größeren Abständen verschieben. Dabei verringert sich der Wert der Wahrscheinlichkeit im Maximum.

Neben dem Hauptmaximum ist wie oben beschrieben in einigen Fällen eine Schulter zu erkennen. In keinem der betrachteten Fälle ist allerdings ein zweites Maximum sichtbar. Insbesondere wäre von Interesse, ob sich unter gewissen Bedingungen ein solches zweites Maximum für einen Abstand in der Nähe des Abstandes nächster Nachbarn, der für Si bei $a_{S i}=2.35 \AA$ [Kit93] liegt, ausbildet. Bei $T=1700 \mathrm{~K}$ und $\Delta t=500$ ps ist dieser Bereich 

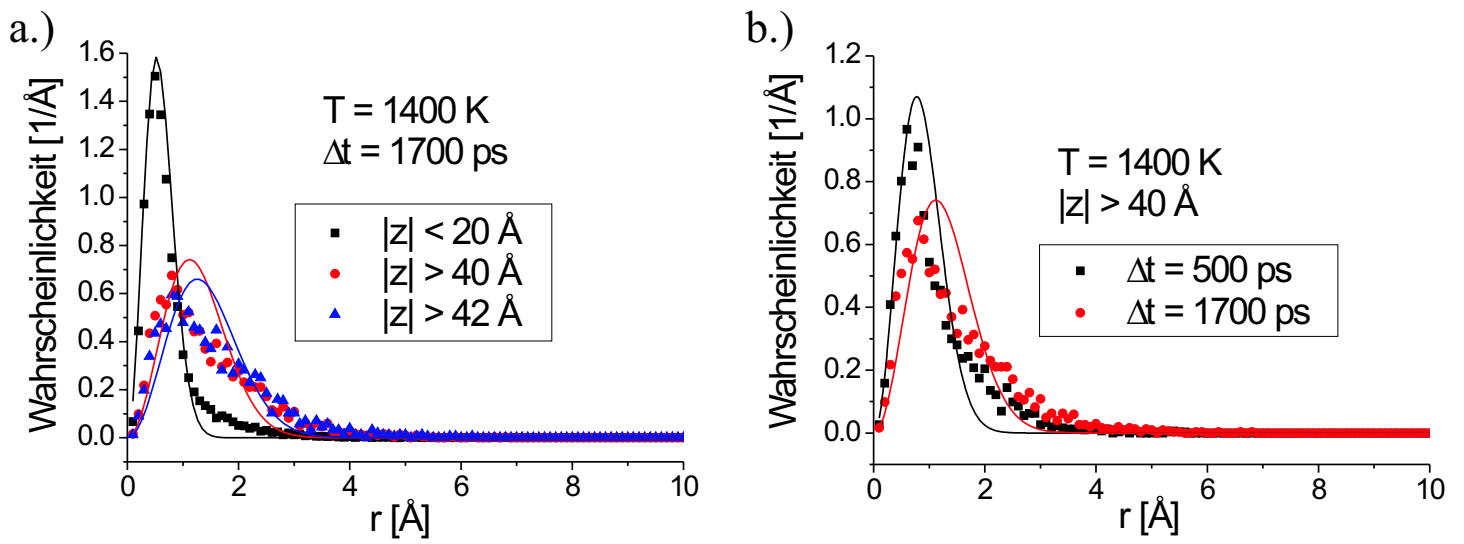

Abbildung 6.18: Histogramme der Verschiebungsweiten bei $T=1400 \mathrm{~K}$ a.) für einen Zeitraum $\Delta t=1700 \mathrm{ps}$ in verschiedenen Bereichen der Probe und b.) für einen Oberflächenbereich als Vergleich zwischen $\Delta t=500 \mathrm{ps}$ und $\Delta t=1700 \mathrm{ps}$.

zumindest für den Oberflächenbereich bereits deutlich vom Hauptmaximum überdeckt. Im Probeninneren befindet sich gerade hier die Schulter auf der Flanke des Hauptpeaks. Bei $T=1400 \mathrm{~K}$ fällt die Schulter auf der Flanke des Hauptpeaks der Atome aus dem Oberflächenbereich mit diesem Abstand zusammen. Bei $T=1000 \mathrm{~K}$ ist die Intensität für diesen Abstand nicht signifikant größer als null.

Da in dieser Arbeit eher die Vorgänge bei tieferen Temperaturen bezogen auf die drei Temperaturbereiche aus Abbildung 6.13 im Mittelpunkt stehen, werden die Vorgänge bei $T=1000 \mathrm{~K}$ und $T=1400 \mathrm{~K}$ nun für längere Zeiten als bisher verfolgt. In Abb. 6.18 sind die Verteilungen der Verschiebungsweiten bei $T=1400 \mathrm{~K}$ für den Zeitraum $\Delta t=1700 \mathrm{ps}$ für das Probeninnere und zwei unterschiedlich dicke Oberflächenbereiche zu sehen. Der dünnere Oberflächenbereich $|z|>42 \AA$, der etwa einer Monolage entspricht ist hier mit hinzu genommen, da in diesem Bereich die höchste Sensitivität auf eventuelle Einzelsprünge an der Oberfläche zu erwarten ist. Für beide Oberflächenbereiche ist die Abweichung von der angepassten Gaußkurve deutlich und im Bereich zwischen $1.7 \AA$ und $2.4 \AA$ existiert jeweils eine relativ stark ausgeprägte Schulter. Damit befindet sich die Schulter im Bereich des Abstandes nächster Nachbarn, der laut [Kit93] bei $a_{S i}=2.35 \AA$ liegt. Die Wahrscheinlichkeit für solche Sprünge liegt hier, wie auch für alle kleineren Abstände, noch oberhalb von $0.2 \AA^{-1}$. Insofern schließt sich die Verteilung im Bereich des Abstandes nächster Nachbarn direkt an den Bereich des Hauptmaximums an, ohne dass eine Trennung beider Bereiche sichtbar ist. Durch die Existenz der Schulter ergibt sich als Fit der Gaußkurve an die Daten eine Kurve, die ihr Maximum bei größeren Abständen hat als die Datenpunkte selbst. Dass dieses Hautpmaximum für den Zeitraum von 1700 ps gegenüber der Verteilung nach 500 ps zu größeren Abständen verschoben ist, ist ebenfalls in Abb. 6.18 anhand der entsprechenden Verteilungen im Oberflächenbereich $|z|>40 \AA$ dargestellt. Da für die Probe bei $T=1000 \mathrm{~K}$ der Diffusionskoeffizient deutlich kleiner 
a.)

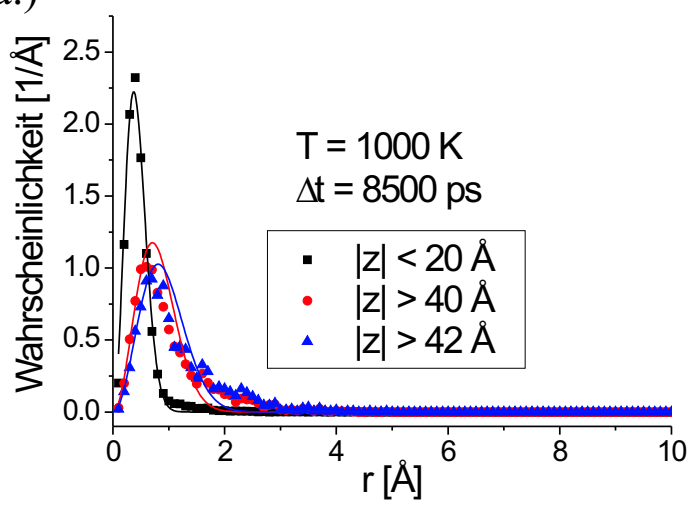

b.)

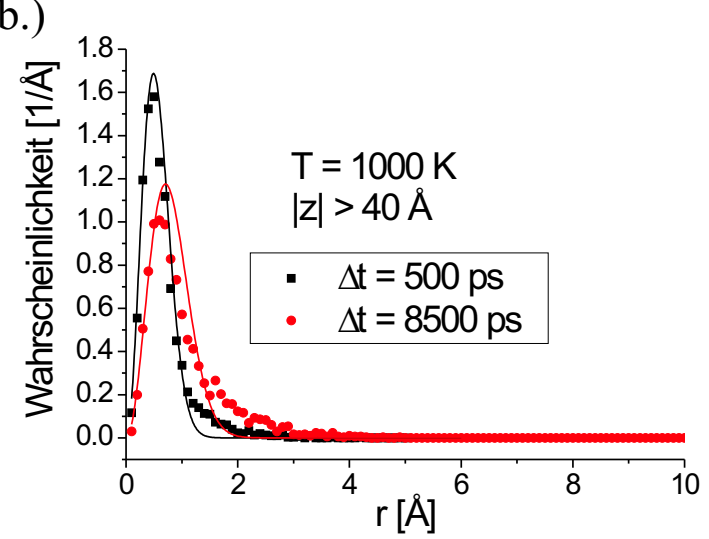

Abbildung 6.19: Histogramme der Verschiebungsweiten bei $T=1000 \mathrm{~K}$ a.) für einen Zeitraum $\Delta t=8500 \mathrm{ps}$ in verschiedenen Bereichen der Probe und b.) für einen Oberflächenbereich als Vergleich zwischen $\Delta t=500 \mathrm{ps}$ und $\Delta t=8500$ ps.

ist, werden die Verschiebungsweiten in Abb. 6.19 in einem Zeitraum von 8500 ps für die verschiedenen Probenbereiche betrachtet und für einen Oberflächenbereich mit den Verschiebungen nach $500 \mathrm{ps}$ verglichen. Hier sind dieselben Tendenzen wie bei $T=1400 \mathrm{~K}$ zu erkennen. Allerdings ist die Schulter in der Verteilung jeweils schwächer ausgeprägt und das Hauptmaximum befindet sich trotz der deutlich längeren Zeit $\Delta t$ noch bei kleineren Abständen. Wie schon bei den anderen untersuchten Temperaturen und Zeitintervallen ergibt sich auch hier kein eindeutiges zweites Maximum.

\subsubsection{Energieverteilung in Si Schichten}

Um die beobachtete Dynamik in amorphen Si Schichten erklären zu können, kann wiederum die Analyse der Energieverteilung hilfreich sein. Daher ist in Abb. 6.20 die Verteilung der potentiellen Energie für die bei $T=1400 \mathrm{~K}$ befindliche Probe nach Abschluss der Berechnungen zu den Verschiebungsweiten dargestellt. Mit eingetragen in den Graphen ist eine angepasste Gauß-Normalverteilung mit den Parametern des Mittelwerts $E_{M}$ und der Standardabweichung $\sigma_{E}$. Hier ist eine deutliche Abweichung von einer Gaußverteilung festzustellen und die Verteilung ist asymmetrisch. Die Asymmetrie spiegelt sich durch die Tatsache wieder, dass die Bedingung $E_{p o t}>E_{M}+2 \sigma_{E}$ von 3085 Atomen erfüllt ist, während $E_{\text {pot }}<E_{M}-2 \sigma_{E}$ nur von 2 Atomen erfüllt ist. Dementsprechend liegt die aus der Simulation bestimmte Anzahl für große (also dem Betrag nach kleine) potentielle Energien oberhalb und für kleine Energien unterhalb der angepassten Gaußkurve. Auf der Seite großer Energien ist eine deutliche Schulter in der Verteilung zu erkennen, die es auf der Seite kleiner Energien nicht gibt. Die Darstellung der Positionen der Atome, für die $E_{\text {pot }}>E_{M}+2 \sigma_{E}$ gilt, zeigt, dass sich diese Atome in verstärktem Maß an der Oberfläche befinden (siehe Abb. 6.20). Allerdings gibt es auch eine erhebliche Anzahl dieser Atome hoher Energie im Probeninneren. Dieser Effekt lässt sich durch die poröse 
a.)

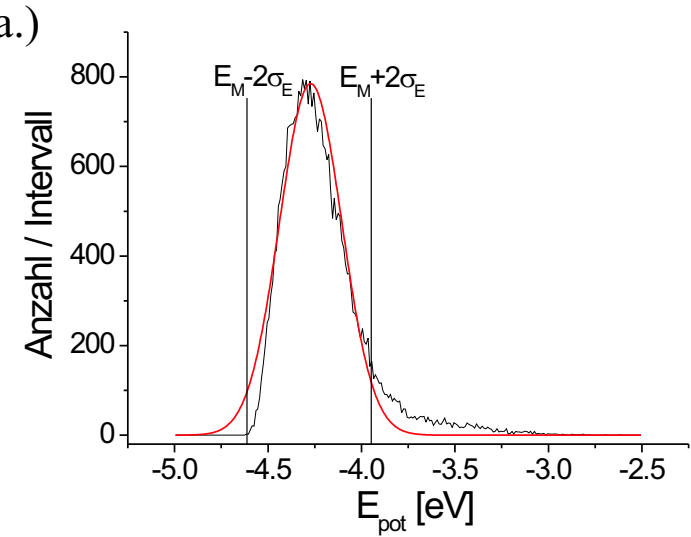

b.)

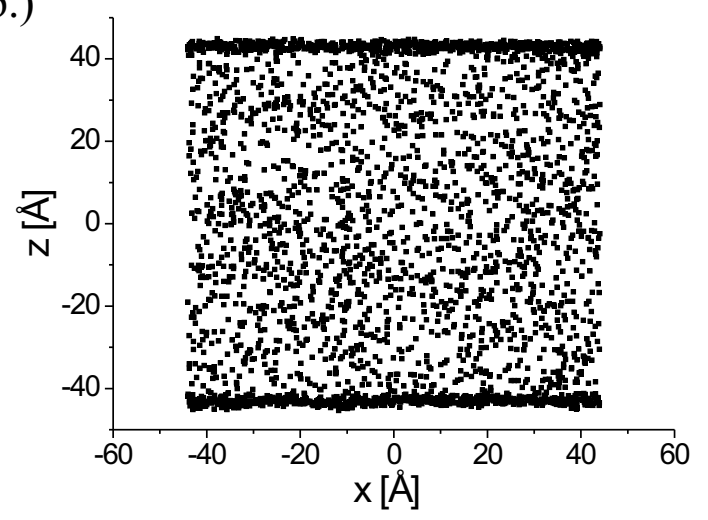

Abbildung 6.20: a.) Histogramm der potentiellen Energien der Atome in der Si Schicht bei $T=1400 \mathrm{~K}$ zum Zeitpunkt nach der Untersuchung aus Abb. 6.18, b.) Position der Atome mit $E_{\text {pot }}>\mu+2 \sigma$ als Projektion auf die xzEbene.

Struktur von Si und damit verbundene innere Oberflächen erklären. Von den 3085 Atomen hoher Energie befinden sich aber 1323 im Oberflächenbereich $|z|>40 \AA$ und damit ist gezeigt, dass die Dichte von Atomen mit hoher Energie im Oberflächenbereich gegenüber dem Probeninneren deutlich erhöht ist. Weiterhin ergibt sich aus diesen Zahlen umgekehrt, dass ein großer Anteil der Oberflächenatome eine gegenüber dem Mittelwert erhöhte Energie hat, denn 1323 Atome im Oberflächenbereich haben eine hohe Energie und die Gesamtzahl der Oberflächenatome lässt sich bei einer Gesamtzahl von $N$ Atomen in der würfelförmigen Probe zu $N_{S}=2 N^{2 / 3}$ abschätzen und das liefert für die verwendete Probe $N_{S}=2048$. Zusammenfassend ergibt sich also bezüglich der energetischen Eigenschaften ein Unterschied zwischen Atomen aus dem Oberflächenbereich und Atomen aus dem Probeninneren.

\subsection{Diskussion des thermisch aktivierten Verhaltens von amorphem Si}

\subsubsection{Probenherstellung und Charakterisierung}

Wie schon beim CuTi ist der Bereich weit oberhalb der Schmelztemperatur im weiteren Verlauf nicht von Interesse, so dass es ausreichend ist, den Verlauf von Volumen und Enthalpie als Funktion der Temperatur unterhalb von $T=4000 \mathrm{~K}$ zu diskutieren, denn oberhalb dieser Temperatur stellt sich lediglich eine ungeordnete Phase ein, in der keine Überreste aus der Struktur, in der sich die Probe zu Beginn bei tiefer Temperatur befand, mehr vorhanden sind. Der Verlauf des Volumens und der Enthalpie als Funktion der Temperatur beim Abkühlen zeigen im relevanten Bereich einen deutlich anderen Verlauf als das in Kap. 6.1 untersuchte metallische Glas CuTi. Beim CuTi 
kommt aus den thermodynamischen Größen nur ein Temperaturbereich als derjenige des Glasübergangs in Frage und der Verlauf der thermodynamischen Größen entspricht qualitativ den in der Literatur häufig angegeben schematischen Graphen zur Darstellung des Glasübergangs [El190; Zar91]. Beim Silizium sind die Verhältnisse komplexer, weil der thermische Ausdehnungskoeffizient für verschiedene Temperaturbereiche verschiedene Vorzeichen hat, wie es sich anhand des wechselnden Vorzeichens der Steigung in der Auftragung des Voluumens als Funktion der Temperatur ergibt. Ein qualitativ ähnliches Verhalten sowohl für das Volumen als auch für die Enthalpie während des Abkühlens aus der Schmelze wird für das Si Tersoff Potential bereits in [CC93] beschrieben, wenngleich dort das $\mathrm{Si}(\mathrm{C})$ Potential mit $\lambda_{3}=0$ anstatt des in dieser Arbeit eingesetzten $\mathrm{Si}(\mathrm{C})$ mit $\lambda_{3} \neq 0$ verwendet wird [Ter88a]. Der Unterschied bezüglich dieses Parameters im Potential wird aber ohnehin als einer mit eher schwachen Auswirkungen diskutiert [Ter88a]. Betrachtet man das Abkühlen aus der Flüssigkeit, so ist die Abnahme des Volumens mit der Abnahme der Temperatur im Bereich hoher Temperaturen zunächst der für einfache Flüssigkeiten erwartete Effekt. Dass sich dann ein Bereich anschließt, in dem mit weiterer Abnahme der Temperatur das Volumen zunimmt, erwartet man dagegen für eine einfache Flüssigkeit nicht. Eine Erklärungsmöglichkeit könnte sein, dass sich in diesem Temperaturbereich die kovalenten Bindungsanteile verstärkt bilden und die Struktur von einer dicht gepackten hin zu einer tetraedrischen beeinflussen. In einer zunehmend tetraedrisch gebundenen Struktur ist die Packungsdichte der Atome nämlich geringer (im „Continuous random network“-Modell für Si ähnlich zur Diamantstruktur: 0.34 [Ell90; Kit93]) als in einer Struktur dicht gepackter Atome ("dense random packingModell': 0.64 [El190]). Hat sich die tetraedrische Struktur voll ausgebildet, sollte es dann wieder eine Abnahme des Volumens bei sinkender Temperatur geben, da sich die Längen der einzelnen Bindungen mit abnehmender Temperatur verkürzen. Diese Interpretation ist auch insofern sinnvoll als sich danach die kovalenten Bindungsanteile verstärkt ab Temperaturen unterhalb des Minimums im Volumen bilden. Dieser Temperaturbereich liegt mit $T=2660 \mathrm{~K}$ in der Nähe der für den Phasenübergang zwischen kristallinem und flüssigem Zustand diskutierten Schmelztemperatur, die in [CC93] als $T_{m}=2547 \pm 22 \mathrm{~K}$ und in [Ter88a] als $T_{m}=3000 \pm 500 \mathrm{~K}$ angegeben wird. Dass sich kovalente Bindungen ausbilden und sich somit eine Netzwerkstruktur bilden kann, würde man allgemein auch nur im festen und nicht im flüssigen Zustand erwarten und diese Erwartung bestätigt sich auch in ab initio Molekulardynamik Rechnungen [SCP91]. Diese Rechnungen zeigen für die flüssige Phase metallisches Verhalten und gegenüber der Tetraederstruktur erhöhte Koordination, während die amorphe Phase tetraedrisch gebunden ist und die elektronische Struktur die eines Halbleiters ist. Insofern ist zu erwarten, dass nach der beschriebenen Interpretation das Minimum im Volumen in der Nähe des Glasübergangs liegt. Durch eine Analyse der Koordinationszahlen konnte außerdem gezeigt werden, dass im festen Zustand bei $T=500 \mathrm{~K}$ über $90 \%$ der Atome die Koordinationszahl 4 haben, während bei $T=2700 \mathrm{~K}$ dieser Anteil bei unter $50 \%$ liegt und auch andere Koordinationen - und zwar vorwiegend höhere - erheblich beitragen [IMM97]. Dieses Ergebnis bestätigt die Ausbildung einer Tetraederstruktur beim Abkühlen von $T=2700 \mathrm{~K}$ hin zu tieferen Temperaturen.

Insofern kann man zwei Beiträge zur Ausdehnung beim Abkühlen diskutieren: Zum 
einen verkürzen sich Bindungen eines Bindungstyps beim Abkühlen und daraus resultiert ein positiver thermischer Ausdehnungskoeffizient. Zum anderen verringert sich beim Übergang von der Flüssigkeit zum Festkörper die durchschnittliche Koordinationszahl und verringert damit die Packungsdichte, so dass sich ein negativer thermischer Ausdehnungskoeffizient ergibt. Der erste Effekt ist für alle Temperaturen relevant und der zweite nur im Bereich des Übergangs vom hochkoordinierten zum niedrigkoordinierten Zustand, so dass sich aus der Überlagerung beider Effekte der Verlauf in Abb. 6.13 qualitativ erklären lässt. In diesem Bild bedeutet das, dass die Enthalpie als Funktion der Temperatur eine höhere Steigung genau in dem Bereich, in dem sich die Koordination ändert, zeigt. Der Bereich, in dem die Strukturänderung stattfindet, zeichnet sich also durch ein geändertes energetisches Verhalten aus. Die Tatsache, dass sowohl das Volumen als auch die Enthalpie keinen abrupten Sprung, der für diskrete Daten einer Unstetigkeit im kontinuierlichen Fall entspricht, zeigen, ist ein Indiz dafür, dass die beobachtete Umwandlung kein Phasenübergang erster Ordnung ist. Damit handelt es sich also nicht um einen Erstarrungsprozess, der dem Übergang aus der Flüssigkeit in den Kristall äquivalent ist. Die Verschmierung des Übergangsbereichs ist eher typisch für einen Glasübergang. Für die Existenz eines Glasübergangs gibt es auch experimentelle Hinweise [HKW04]. Die zitierte Arbeit diskutiert weiterhin einen Übergang aus einer Flüssigkeit hoher Dichte zu einer festen amorphen Phase niedriger Dichte mit einem Glasübergang. Insofern kann bis hierhin festgehalten werden, dass ein Glasübergang bei $T_{1}, T_{2}$ oder im dazwischenliegenden Bereich (vgl. Abb. 6.13) stattfindet. Eine weitere Eingrenzung des Temperaturübergangsbereichs wird sich im Folgenden noch ergeben.

Dafür wurde die durch rasches Abkühlen hergestellte Bulk-Probe mit gleicher Rate geheizt und währenddessen eine Zugspannung von $p=5 \mathrm{kbar}$ in $\mathrm{x}$-Richtung angelegt. Beim Glasübergang ist dann zu erwarten, dass die Probe beginnt, sich in x-Richtung auszudehnen und wegen Volumenerhaltung in den dazu senkrechten Richtungen zusammenzuziehen, weil im Bereich des Glasübergangs die Viskosität stark abnimmt. Diesen Effekt gäbe es auch, wenn das System keinen Glasübergang durchlaufen sondern schmelzen würde. Wenn es aber einen Glasübergang gibt, kann mit diesem Experiment die (auf den hier betrachteten Zeitskalen) relevante Übergangstemperatur bestimmt werden. Das Ergebnis der Simulation ist in Abb. $6.21 \mathrm{zu}$ sehen. Der beschriebene Prozess setzt etwa bei $T=2400 \mathrm{~K}$ ein. Oberhalb dieser Temperatur ändert sich die Steigung der Ausdehnung in x-Richtung über einen sehr großen Temperaturbereich. Als Ergebnis daraus ist festzuhalten, dass der Glasübergang nicht unterhalb von $T=2400 \mathrm{~K}$ stattfindet. Weiterhin zeigt sich hier der Übergang ungefähr in der Temperaturregion, in der beim Abkühlen das Volumen als Funktion der Temperatur ein Minimum zeigt. Daher lässt sich übereinstimmend argumentieren, dass in diesem Temperaturbereich beim Abkühlen die Bildung kovalenter Bindungen einsetzt und beim Heizen diese Bindungen in einem solchen Maße wieder aufgelöst sind, dass Fließen möglich ist. Dementsprechend sollte $T_{g}$ in diesem Bereich liegen.

Die Strukturanalyse mittels Paarkorrelationsfunktion (siehe Kap. 6.3.1.2) zeigt, dass bei allen untersuchten Proben eine Struktur ohne langreichweitige Ordnung vorliegt, denn für Abstände oberhalb von $9 \AA$ nähern sich die Paarkorrelationsfunktionen schon sehr gut dem konstanten Grenzwert. Außerdem existieren bei einigen Abständen, für die 


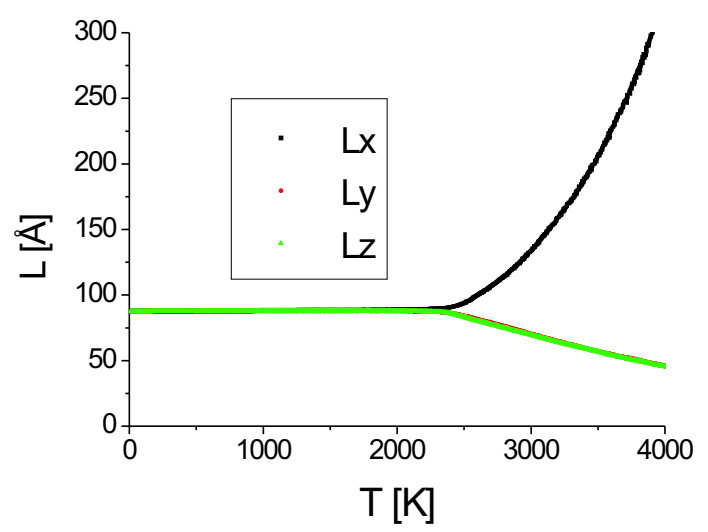

Abbildung 6.21: Kantenlänge $L$ der Simulationsbox in den drei Raumrichtungen während des Heizprozesses einer zu Beginn amorphen Si Probe mit angelegter Zugspannung von $p=5$ kbar in $\mathrm{x}$-Richtung.

in einer kristallinen Struktur Peaks erwartet werden, keine Peaks [LL88]. Das bedeutet, dass sämtliche Proben nicht kristallin sind. Des Weiteren stimmen die Paarkorrelationsfunktionen bezüglich ihrer qualitativen Struktur und der Position der Maxima und Minima mit Ergebnissen aus Neutronenstreuexperimenten an amorphen Si Schichten überein [FL89]. Die Breite ist zum einen durch die statistische Anordnung der Atome und zum anderen durch die thermischen Schwingungen dieser Atome bedingt. Wegen des ersten Beitrags sind die Peaks auch bei sehr tiefen Temperaturen sehr viel breiter als es bei kristallinen Strukturen der Fall ist. Die Verbreiterung der Peaks bei gleichzeitiger Abnahme der Intensitätsmaxima mit steigender Temperatur lässt sich durch verstärkte thermische Schwingungen, die die mittlere Abweichung von der Gleichgewichtsposition der Atome erhöhen, begründen. Als einzige der betrachteten Proben zeigt die bei $T=10 \mathrm{~K}$ ein Nebenmaximum im Bereich des zweiten Hauptminimums und außerdem Abweichungen im ersten Hauptminimum (siehe Abb. 6.14). Diese kleinen Nebenmaxima bzw. Abweichungen sind auch aus Neutronenstreuexperimenten an amorphem Si bekannt $\left[\mathrm{KMP}^{+} 89\right]$. Ansonsten fällt auf, dass bei $T=2200 \mathrm{~K}$ die Ordnung schon bei kürzeren Abständen verloren geht als es bei $T=10 \mathrm{~K}$ und $T=1400 \mathrm{~K}$, die sich diesbezüglich untereinander ähnlich verhalten, der Fall ist. Außerdem geht die Intensität sowohl bei $T=10 \mathrm{~K}$ als auch bei $T=1400 \mathrm{~K}$ im ersten Minimum fast auf null zurück, während bei $T=2200 \mathrm{~K}$ hier der Wert deutlich größer ist. Dieses Ergebnis passt damit zusammen, dass - zumindest beim Kühlen - gerade zwischen $T=1400 \mathrm{~K}$ und $T=2200 \mathrm{~K}$ der thermische Ausdehnungskoeffizient negativ ist und dieser Effekt lässt sich wie zuvor dargestellt durch die Erhöhung der Koordination erklären. Wenn die Tetraederstruktur verloren geht, gilt das auch für die Ordnung im Bereich des für das erste Minimum relevanten Abstands. Die Herstellung der Oberflächen bewirkt keine sichtbare Änderung der Paarkorrelationsfunktion gegenüber der Bulk-Probe, so dass auch für den Oberflächenbereich eine Kristallisation ausgeschlossen werden kann. Somit ist anhand der 
Strukturanalyse sichergestellt, dass die untersuchten Proben jeweils eine ungeordnete Struktur haben.

Insgesamt hat sich in diesem Kapitel gezeigt, dass mit den beschriebenen Methoden dünne Schichten glasartigen Siliziums hergestellt werden können.

\subsubsection{Dynamik dünner Si Schichten}

Die Tiefenprofile der Diffusionskoeffizienten in Abb. 6.16 zeigen beim Silizium, wie bereits für die zuvor besprochenen CuTi Schichten, einen deutlichen Oberflächeneffekt, indem der Wert des Diffusionskoeffizienten im Oberflächenbereich gegenüber dem Probeninneren deutlich ansteigt. Dass mit steigender Temperatur der Wert des Diffusionskoeffizienten für alle Probenbereiche ansteigt, ist zu erwarten, da es sich bei der Diffusion um einen thermisch aktivierten Prozess handelt. Die Zunahme des Einflussbereichs der Oberfläche, könnte sich dadurch begründen lassen, dass die Beweglichkeit bei der tiefsten Temperatur im Probeninneren so klein ist, dass kein wesentlicher Transport stattfinden kann und merklicher Transport nur an der Oberfläche stattfindet. Bei erhöhter Temperatur wird auch Bewegung für Atome, die weiter von einer Oberfläche entfernt sind, möglich. Es gilt aber weiterhin, dass die Beweglichkeit an der Oberfläche erhöht ist, weil weniger Nachbarn vorhanden sind. Dass sich die Tiefenprofile der Diffusionskoeffizienten auch für die Si Schichten durch Gleichung (6.6) beschreiben lassen (siehe Abb. 6.16, Tab. 6.4), untermauert die Aussage, dass es sich bei der Auswirkung der Oberfläche auf die Dynamik um einen sehr allgemeinen thermodynamischen Effekt handelt. Die allgemeine Form der Gleichung ist nämlich unabhängig vom Material und materialspezifische Eigenschaften gehen nur über die Konstanten ein. Trotzdem beschreibt sie die Ergebnisse für verschiedene Materialien, wie hier einen Halbleiter und zuvor ein Metall (siehe Kap. 6.1.2), korrekt.

Dass die Histogramme der Verschiebungsweiten sich für konstante Zeiten mit wachsender Temperatur zu größeren Verschiebungsweiten verlagern (siehe Abb. 6.17), entspricht in jedem Fall der Erwartung, unabhängig davon ob der Grund für die Verschiebungen in thermischen Schwingungen oder in Transportprozessen liegt. Im Oberflächenbereich gibt es bei $T=1400 \mathrm{~K}$ und $T=1700 \mathrm{~K}$ bereits nach $\Delta t=500$ ps Verschiebungen mit einer Weite im Bereich des Abstands nächster Nachbarn. Diese Verschiebungsweiten gehen über die für thermische Schwingungen typische hinaus und es stellt sich nun wiederum die Frage, welcher Prozess für diesen Transport verantwortlich ist. Wie bereits in Kap. 6.1.3 erwähnt, spricht eine reine Gaußverteilung für einen kooperativen Prozess, während sich Einzelsprünge durch Maxima bei den atomaren Abständen auszeichnen. Natürlich können auch beide Arten von Prozessen beitragen und sich somit überlagern. Dass kann dann beispielsweise dazu führen, dass zusätzliche durch Einzelsprünge hervorgerufene Maxima durch das auf kooperativen Prozessen beruhende Hauptmaximum überdeckt werden. Nach $\Delta t=500$ ps ist zwar bei keiner Temperatur und in keinem Probenbereich ein zweites Maximum beim Abstand nächster Nachbarn zu erkennen, aber das heißt im Umkehrschluss eben nicht eindeutig, dass keine Einzelsprünge stattfinden. Bei $T=1000 \mathrm{~K}$ und $T=1400 \mathrm{~K}$ ist nach $\Delta t=500$ ps die Intensität im Bereich der Entfernung für Einzelsprünge an der Oberfläche zu niedrig, um verlässliche Aussagen machen 
zu können. Bei der Untersuchung längerer Zeiträume ergibt sich beim Abstand nächster Nachbarn zwar eine leichte Erhöhung in der Intensität und die Existenz der Schulter ist weiter deutlich, aber ein zweites Maximum tritt nicht auf. Die Untersuchungen bei diesen Temperaturen über längere Zeiten, zeigen durch die allgemeine Verschiebung der entsprechenden Verschiebungsweiten zu größeren Abständen übrigens erneut, dass zu diesen Histogrammen tatsächlich Transportprozesse und nicht nur thermische Schwingungen beitragen. Bei rein thermischen Schwingungen sollte nämlich keine Zeitabhängigkeit der Verteilung bestehen, sofern die Zeiten deutlich oberhalb der Schwingungsdauer thermischer Schwingungen sind, was hier wiederum für beide Untersuchungszeiten gilt. Bei einer weiteren deutlichen Verlängerung der Beobachtungszeit, würde dann irgendwann der Hauptpeak den Bereich des Abstandes nächster Nachbarn überdecken, wie es sich bei $T=1400 \mathrm{~K}$ und $\Delta t=1700 \mathrm{ps}$ andeutet und bei $T=1700 \mathrm{~K}$ bereits bei $\Delta t=500 \mathrm{ps}$ der Fall ist. Insgesamt muss aber festgehalten werden, dass unter Bedingungen, bei denen die Beweglichkeit groß genug ist und der Hauptpeak nicht im Bereich des Abstands nächster Nachbarn liegt, eine Schulter beim Abstand nächster Nachbarn vorhanden ist. Insofern tragen hier auch Sprünge im Bereich des Nachbarabstandes zum Transport bei. Andererseits handelt es sich nicht um so deutlich ausgeprägte Einzelsprünge, wie sie für $\mathrm{Cu}$ Atome auf CuTi Oberflächen beobachtet werden (siehe Kap. 6.1). Aufgrund der porösen Netzwerkstruktur der bei $p=0$ mbar hergestellten Si Probe ist auch denkbar, dass die Entfernung bei Einzelsprüngen vergleichsweise stark um einen Mittelwert des Abstands nächster Nachbarn streut, weil die Abstände atomarer Positionen in einer solchen porösen Struktur größeren Streuungen unterworfen sind als es in einer dicht gepackten Struktur gilt. Weiterhin ist im amorphen kovalent gebundenen System auch eine Erklärungsmöglichkeit für Abweichungen von einer Sprunglänge, die dem Abstand nächster Nachbarn entspricht, dadurch gegeben, dass sich durch Änderung der atomaren Positionen auch die kovalenten Bindungsverhältnisse ändern. Im Sinne des „Continuous random networks" Strukturmodells [Ell90] gilt zwar die tetraedrische Anordnung bezüglich der Nahordnung als die energetisch günstigste, aber die relative Orientierung dieser tetraedrischen Strukturen untereinander ist nicht streng geordnet wie im Kristall. Wechseln nun kovalente Bindungen aufgrund kleiner Änderungen atomarer Positionen ihre atomaren Bindungspartner, so sind für die tetraedrische Nahordnung eventuell kleinere Korrekturen der atomaren Positionen nötig. Diesen Vorgang kann man sich bildlich als „Wackeln“ des Netzwerks vorstellen. Wenn sich solche kleinen Bewegungen an einen atomaren Einzelsprung anschließen, führt das dazu, dass die entsprechende Sprungweite statistisch um den durchschnittlichen Abstand nächster Nachbarn verteilt ist.

Die Tatsache, dass (insbesondere bei ausreichend langen Zeiten) bei $T=1400 \mathrm{~K}$ die Verteilung der Verschiebungsweiten deutliche Abweichungen von einer Gaußverteilung zeigen, ist ein Indiz dafür, dass sich das System im Glaszustand befindet und nicht eine Flüssigkeit ist. Für eine Flüssigkeit sind nämlich Transportprozese ohne ausgezeichnete Längenskala zu erwarten, die wiederum eine perfekte Gaußkurve im Histogramm bedingen würden. Insofern spricht ebenso die Abweichung des Histogramms für die Atome im Probeninneren bei $T=1700 \mathrm{~K}$ dafür, dass sich das System unterhalb der (Bulk)-Glastemperatur befindet. Dass die Abweichungen von einer Gaußkurve für die Oberflächenatome bei dieser Temperatur nicht so groß sind, lässt sich dadurch er- 
klären, dass das Hauptmaximum und ein eventuelles Nebenmaximum ungefähr an der gleichen Stelle liegen, so dass keine große Abweichung von der Gaußverteilung entstehen kann. Ein weiterer Erklärungsansatz wäre, dass für die Oberfläche die Glastemperatur einen kleineren Wert hat als es für eine Bulk-Probe bzw. das Probeninnere gilt und daher bereits flüssiges Verhalten an der Oberfläche stattfindet. Dass die erhöhte Mobilität an der Oberfläche mit einer erniedrigten kalorischen Glastemperatur $T_{g}$ in Verbindung steht, während die Übergangstemperatur $T_{c}$ des dynamischen Glasübergangs im Sinne der Modenkopplungstheorie sich allerdings nicht wesentlich ändert, wird z.B. in [Böd99] ausführlich diskutiert. Weil aber $T_{c}$ den Übergang zum flüssigen Verhalten beschreibt, scheint dieser Erklärungsansatz somit nicht der richtige zu sein.

Die Analyse der Energieverteilung in der Si Probe bei $T=1400 \mathrm{~K}$ entspricht der Erwartung, indem sich Oberflächenatome energetisch auf höherem Niveau befinden als die Atome im Probeninneren. Durch diesen Unterschied ergibt sich nämlich eine von null verschiedene Oberflächenenergie für das System. Weiterhin passen diese energetischen Eigenschaften zu den Ergebnissen aus den Untersuchungen der Dynamik, weil sich dort eine erhöhte Beweglichkeit im Oberflächenbereich ergibt. Eine solche erhöhte Beweglichkeit lässt sich dadurch erklären, dass die Atome energetisch nicht so stark gebunden sind wie die Atome aus dem Probeninneren.

\subsection{Zusammenfassende Diskussion}

Für beide untersuchten Materialien konnte gezeigt werden, dass in der MD-Simulation amorphe dünne Schichten hergestellt worden sind. Unterschiede zwischen beiden Materialien spiegeln sich in den strukturellen Eigenschaften wieder und äußern sich auch durch das thermodynamische Verhalten während der Herstellung.

Nahezu identisch ist für beide Materialien der Einfluss der Oberfläche auf das Verhalten des Diffusionskoeffizienten, der jeweils im Bereich der Oberfläche gegenüber dem Probeninneren ansteigt. Jedoch ist der Mechanismus, dem die atomaren Bewegungen zugrunde liegen, für die untersuchten Systeme verschieden, denn während im CuTi die $\mathrm{Cu}$ Atome an der Oberfläche recht deutlich die Existenz von atomaren Einzelsprüngen zeigen, ist ein solcher Mechanismus für die Si Oberflächen nicht erkennbar.

Es sollte erwähnt werden, dass mit der hier verwendeten Methode zur Berechnung der Diffusionskoeffizienten insbesondere bei Temperaturen deutlich unterhalb der Glastemperatur, tendenziell zu große Werte resultieren. Der Grund dafür ist, dass alle atomaren Verschiebungen in die Berechnung der Diffusionskoeffizienten eingehen. Daher sind also auch die atomaren Verschiebungen, die aus thermischen Schwingungen resultieren und nicht zu einem Transportprozess beitragen, berücksichtigt. Die mittleren quadratischen Amplituden thermischer Schwingungen $\left\langle\Delta \vec{x}_{T}^{2}\right\rangle$ fallen aber sehr viel schwächer als Funktion der Temperatur ab als die Verschiebungsquadrate $\left\langle\Delta \vec{x}_{D}^{2}\right\rangle$, die zur thermisch aktivierten Diffusion beitragen $\left(\left\langle\Delta \vec{x}_{T}^{2}\right\rangle \propto T,\left\langle\Delta \vec{x}_{D}^{2}\right\rangle \propto \exp \left(-\frac{H}{k_{B} T}\right)\right)$. Deshalb ergeben die bei verschiedenen Temperaturen berechneten Diffusionskoeffizienten für Si auch insbesondere bei tiefen Temperaturen Abweichungen vom Arrhenius-Verhalten. Diese Schwierigkeiten treten nicht auf, wenn man tatsächlich den Grenzfall $\Delta t \rightarrow \infty$ bei 
der Berechnung der Diffusionskoeffizienten betrachten würde, doch das ist in der MDSimulation prinzipiell nicht möglich, wenngleich längere Simulationszeiten den Fehler verringern.

Um den Einfluss der thermischen Schwingungen auf den Wert des Diffusionskoeffizienten zu verhindern, kann man einen Abstand $r_{c}$ einführen und nur solche atomaren Verschiebungen berücksichtigen, die diesen Wert überschreiten [KS04]. Der Wert für $r_{c}$ muss so gewählt werden, dass thermische Schwingungen eine kleinere und atomarer Transport eine größere Verschiebung bewirken. Während im Kristall ein entsprechender Wert für $r_{c}$ recht einfach festzulegen ist, gilt dass im Amorphen wiederum nicht, weil Transportprozesse kollektiv ablaufen können und es somit keine untere Grenze für eine atomare Verschiebungsweite bei Transportprozessen gibt. Somit gestaltet sich die Wahl des Parameters im Amorphen als relativ komplex [KS04]. Es wurden auch Tests zu Berechnungen von Diffusionskoeffizienten unter Berücksichtigung des beschriebenen Kriteriums durchgeführt, wobei dort der Wert von $r_{c}$ aus den thermischen Schwingungsamplituden bestimmt worden ist.

Andere Methoden zur Vermeidung des Einflusses thermischer Schwingungen auf die Diffusionskoeffizienten sind die Mittelung atomarer Positionen über den Zeitraum mehrerer Schwingungsperioden [KS04] oder ein rasches Abkühlen, durch das sich alle Atome in ihre Gleichgewichtsposition bewegen [Tei92]. Letztgenannte Methode bewirkt allerdings das Auftreten von Verzerrungen und ist daher nicht ohne Einschränkung geeignet.

Des Weiteren sind für die Diffusionskoeffizienten umso größere Werte zu erwarten, je kleiner die Relaxationszeiten vor der Diffusionsuntersuchung sind [FFM $\left.{ }^{+} 03\right]$. Somit sind aus den durchgeführten Simulationen größere Werte als in entsprechenden experimentellen Untersuchungen zu erwarten.

Da in dieser Arbeit aber nicht die absoluten Werte der Diffusionskoeffizienten im Vordergrund stehen, sondern die relativen Einflüsse von Oberflächen, und da die Festlegung des Parameters $r_{c}$ nicht eindeutig ist, wird in der Regel in dieser Arbeit das beschriebene Kriterium nicht verwendet. Genauso ist deshalb der Einsatz der anderen Methoden zur Vermeidung des Einflusses thermischer Schwingungen für die untersuchten Fragestellungen nicht notwendig und auch Einfluss der Relaxationszeiten ist nicht sehr kritisch, solange die relativen Unterschiede von Probeninnerem und Probenoberfläche untersucht werden. Vor allem die charakteristischen Merkmale in den Histogrammen der Verschiebungsweiten im Bereich des Abstands nächster Nachbarn sind nicht durch thermische Schwingungen beeinflusst, weil die Amplituden thermischer Schwingungen wesentlich kleiner als die Abstände nächster Nachbarn sind. 


\section{Bestrahlungsinduzierte Prozesse in der MD-Simulation}

In diesem Kapitel werden die Ergebnisse zu den durch atomaren Beschuss induzierten Prozessen, die im Rahmen von MD-Simulationen gewonnen werden konnten, dargestellt. Wie schon im Kapitel über thermisch aktivierte Prozesse in der MD-Simulation, werden auch hier die Untersuchungen sowohl für das metallische Glas CuTi (in der Zusammensetzung $\mathrm{Cu}_{50} \mathrm{Ti}_{50}$ ) als auch für das kovalent gebundene Glas Si durchgeführt. Eine zusammenfassende Darstellung der wesentlichen Ergebnisse dieses Kapitels findet sich in [VM07].

Das Ziel der MD-Simulationen zur Bestrahlung ist die Identifikation und Abgrenzung der für den atomaren Transport wichtigen Prozesse. Um dieses Ziel zu erreichen, werden verschiedene Ansätze gewählt. Zunächst werden die Eigenschaften der atomaren Dynamik mit Hilfe statistischer Analysen, wie der bereits bei der Untersuchung thermisch aktivierter Prozesse verwendeten Verteilung atomarer Verschiebungsweiten, untersucht. Mit dieser Methode können bereits Ergebnisse bezüglich der Art der bestrahlungsinduzierten Dynamik gewonnen werden. Im weiteren Verlauf wird dann die quantitative Analyse der im Rahmen der Kontinuumsmodelle in Kap. 4 diskutierten Terme, die jeweils einen physikalischen Prozess während der Bestrahlung beschreiben, im Mittelpunkt stehen. Dabei wird es darum gehen, die Koeffizienten der verschiedenen Terme durch die Analyse der aus der MD-Simulation gewonnenen Daten möglichst realistisch zu berechnen. Mit diesen Ergebnissen kann das relative Gewicht der verschiedenen Prozesse quantitativ berechnet werden. Diese Methode beruht also auf einer Kombination von Modellen auf mesoskopischer Skala (Kontinuumsmodelle) und einer Analyse der Prozesse auf atomistischer Skala (MD-Simulation). Im Mittelpunkt steht bei den Untersuchungen die Analyse der glättenden Prozesse, denn die experimentellen Ergebnisse in Kap. 5 zeigen, dass unter den gegebenen Bedingungen die Glättungseffekte dominieren.

Auch wenn Untersuchungen zu verschiedenen Bestrahlungsenergien durchgeführt worden sind, wird das Hauptaugenmerk im Folgenden auf der Bestrahlungsenergie $1 \mathrm{keV}$ liegen. Diese Energie ist von derselben Größenordnung wie die im experimentellen Teil der Arbeit verwendeten. Die Herstellung und Bestrahlung der dünnen Schichten erfolgt wie in Kap. 3.4 beschrieben.

Als Umgebungstemperaturen während der Bestrahlung werden zwei verschiedene Temperaturen untersucht, nämlich $T=10 \mathrm{~K}$ und $T=300 \mathrm{~K}$. Dabei sind mit Umgebungstemperaturen die Temperaturen gemeint, die an den Rändern der Temperaturkontrolle entsprechend vorgegeben werden (siehe Kap. 3.4). Simulationen bei $T=10 \mathrm{~K}$ haben den Vorteil, dass sich die strahlungsinduzierten Prozesse nicht mit thermisch aktivierten überlagern. Das erleichtert die direkte Analyse der strahlungsinduzierten Prozesse. Si- 
mulationen bei einer Umgebungstemperatur von $T=300 \mathrm{~K}$, also bei Raumtemperatur, haben wiederum den Vorteil, dass sie die in vielen Experimenten gültigen Bedingungen (so auch die im experimentellen Teil dieser Arbeit geltenden) wiedergeben.

Um Aussagen von statistischer Relevanz erlangen zu können, ist eine Vielzahl von Einschüssen nötig. Je nach der für eine bestimmte Fragestellung nötigen Anzahl werden direkt verschiedene Startpositionen des einzuschießenden Atoms gewählt oder die Startposition wird in einem automatisierten Verfahren (mit einem Computercode von S. G. Mayr) zufällig gewählt.

Die genauen Analysemethoden werden an gegebener Stelle erläutert und im Zusammenhang mit den Ergebnissen für CuTi vorgestellt. Die mit denselben Analysemethoden gewonnenen Ergebnisse für Si werden daran anschließend wiedergegeben.

Die Eigenschaften der in der MD-Simulation verwendeten Potentiale sowie weitere Details der Simulationstechnik sind in Kap. 3 beschrieben.

\subsection{Bestrahlung von amorphen CuTi Oberflächen}

In diesem Abschnitt geht es um die Bestrahlung von gläsernen CuTi Oberflächen, die wie in Kap. 3.4 beschrieben hergestellt werden. Nach der Relaxation der Volumenproben (d.h. periodische Randbedingungen für alle Richungen), werden durch Aufheben der periodischen Randbedingungen in z-Richtung zwei Oberflächen hergestellt, und unter diesen Bedingungen wird die Probe solange relaxiert, bis die Energie des Systems sich als Funktion der Zeit einem konstanten Wert genähert hat.

\subsubsection{Allgemeine Phänomene}

Begriffe und Erwartungen Beim Einschuss eines Atoms auf eine Oberfläche überträgt sich die kinetische Energie des ankommenden Atoms auf die Atome der beschossenen Schicht. Dabei ist bekannt, dass sich zunächst ballistische Stoßkaskaden entwickeln, bei denen einzelne Atome angestoßen werden und sich mit hoher kinetischer Energie in der Probe bewegen [SK56; AR98]. In dieser Phase entspricht die kinetische Temperatur, die aus den Geschwindigkeiten der Atome berechnet wird, nicht einer thermodynamisch sinnvoll definierten Temperatur, weil das System sehr weit von einem Gleichgewichtszustand entfernt ist. Nach einer gewissen Zeit verteilt sich die vorhandene Energie auf eine deutlich größere Anzahl von Atomen, so dass sich aufgeschmolzene Bereiche („thermal spikes") bilden können, die wiederum nach weiterer Zeit erstarren [SK56; AR98]. Außer den Anregungen im "thermal spike“ ist davon auszugehen, dass einige Atome aus der Oberfläche herausgeschlagen werden („Sputtern“) und zusätzlich Atome im Bereich der Oberfläche räumlich und energetisch angehoben werden, so dass sie danach eine höhere Beweglichkeit aufweisen („Adatome“) [AR98].

Im verwendeten Simulationscode ist die Möglichkeit enthalten, die kinetische Energie der Atome zu analysieren, um so einen Einblick zu gewinnen, ob es einen flüssigen Bereich gibt oder nicht. Die Flüssigkeitsanalyse wird in Intervallen einer wählbaren Zahl von Integrationsschritten durchgeführt [NA97; $\mathrm{NGA}^{+}$98]. Dabei werden die Atome als 
flüssig bezeichnet, bei denen ihre eigene kinetische Energie und die ihrer nächsten Nachbarn eine vorgebbare Energiegrenze überschreiten [NA97; NGA ${ }^{+} 98$ ]. Diese Energiegrenze entspricht über die Beziehung $E=\frac{3}{2} k_{B} T$ der Temperatur, bei der das System vom festen zu einem flüssigen Zustand übergeht und für diese Temperatur wird hier die Glastemperatur $T_{g}$ verwendet. Dabei muss allerdings immer berücksichtigt werden, dass die Glastemperatur von der Kühl- bzw. Heizrate abhängig ist. Es werden die in Kap. 6 berechneten Werte für die Glastemperatur aus dem Kühlprozess eingesetzt. Das ist deshalb physikalisch sinnvoll, weil die Kühlraten während der Probenherstellung ( $\dot{T}=20 \mathrm{~K} / \mathrm{ps}$ ) von ähnlicher Größenordnung sind wie die lokalen Kühlraten in einem aufgeschmolzenen Bereich nach dem Einschuss eines Atoms. Diese Tatsache wird im folgenden Ergebnisteil durch die Resultate der MD-Simulationen bestätigt werden. Zu beachten ist, dass sich die Eigenschaft flüssig in diesem Abschnitt immer auf die oben vorgestellte kinetische Definition bezieht. Die Anzahl der flüssigen Atome kann als Funktion der Zeit dargestellt werden, so dass ein Eindruck von der Anzahl flüssiger Atome im „thermal spike“ $N_{l i q}$ und von der Lebensdauer des „thermal spikes“ $\tau_{l i q}$ gewonnen werden kann. Mit angemessenen Kriterien können beide Größen berechnet werden (s.u.). Für feste Zeitpunkte können alle flüssigen Atome im Ortsraum dargestellt werden, so dass ein Eindruck von der räumlichen Ausdehnung des „thermal spike" vermittelt wird und diese Ausdehnung auch quantitativ bestimmt werden kann. Die Ausdehnung in z-Richtung, also entlang der Oberflächennormalen, ist insbesondere im Zusammenhang mit den Kontinuumskonzepten (siehe Kap. 4.5.4) von Bedeutung. Größen wie die Lebensdauer oder auch die räumliche Ausdehnung der „thermal spikes“ sind abhängig von der Einschussenergie und der gewählten Umgebungstemperatur. Durch Lösung der Wärmeleitungsgleichung ergibt sich, dass die Lebensdauer eines kugelförmigen „thermal spikes“ mit der Einschussenergie $E$ gemäß $\tau_{\text {liq }} \propto E^{2 / 3}$ und die eines zylindrischen „thermal spikes" gemäß $\tau_{l i q} \propto E$ skaliert [SK56; AR98]. Für die Anzahl flüssiger Atome sollte immer $N_{l i q} \propto E$ gelten, weil pro Atom eine feste Mindestenergie benötigt wird, um die kinetische Energie soweit anzuheben, dass es als flüssiges Atom gelten kann.

Die Anzahl gesputterter Atome entspricht der Anzahl von Atomen, die sich von der Probe getrennt haben. Auf die Berechnung der Anzahl von Adatomen wird an späterer Stelle in Kap. 7.1.3.2 eingegangen.

Ergebnisse Beim Einschuss eines $\mathrm{Cu}$ Atoms der Energie $E=1 \mathrm{keV}$ auf eine wie in Kap. 3.4 beschrieben hergestellte CuTi Schicht mit einer Umgebungstemperatur von $T=10 \mathrm{~K}$ ergibt sich ein typischer Verlauf für die Anzahl von (im Sinne der zuvor gegebenen Definition) flüssigen Atomen als Funktion der Zeit, wenn über mehrere Einschüsse mit verschiedenen Startpositionen gemittelt wird. Als Repräsentant ist der Verlauf für einen einzelnen Einschuss in Abb. 7.1 dargestellt. Nach dem steilen Anstieg zu Beginn ergibt sich ein Maximum. Im Anschluss daran bildet sich ein Plateau aus, in dem die Anzahl für einige Zeit konstant bleibt und unterhalb des Wertes im Maximum liegt. Nach einiger Zeit zerfällt dieses Plateau, bis am Ende keine flüssigen Atome mehr vorhanden sind. Bei diesem Verlauf ist zu beachten, dass während des Anstiegs und im Bereich des Maximums gerichtete Geschwindigkeiten auftreten und dementsprechend noch keine 
a.)

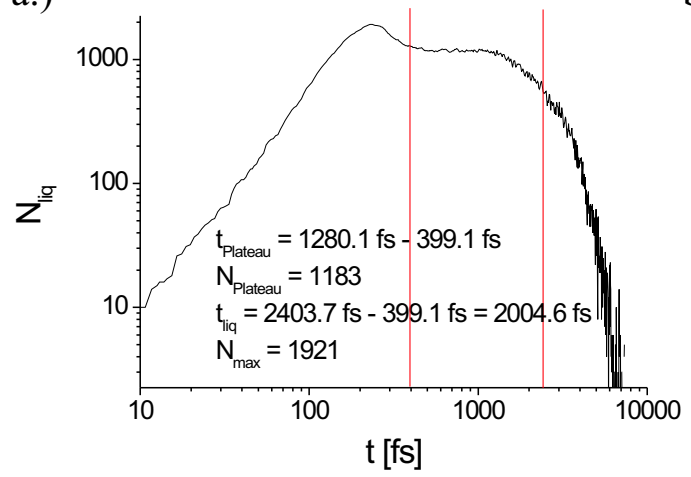

b.)

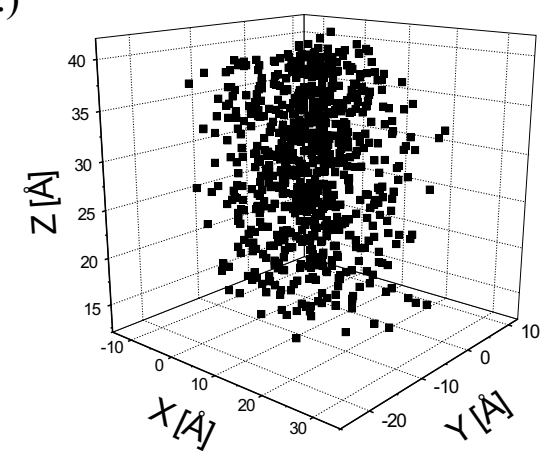

Abbildung 7.1: a.) Anzahl flüssiger Atome als Funktion der Zeit (Die roten Linien markieren zeitlich den Anfangs- und Endpunkt des flüssigen Bereichs.) und b.) räumliche Anordnung der flüssigen Atome zum Zeitpunkt $t=824 \mathrm{fs}$, jeweils für einen $\mathrm{Cu}$ Einschuss der Energie $E=1 \mathrm{keV}$ auf $\mathrm{CuTi}$ bei $T=10 \mathrm{~K}$ Umgebungstemperatur.

Temperatur definiert ist.

Im Plateau wird der durchschnittliche Wert der Anzahl flüssiger Atome bestimmt und liefert somit den Wert für $N_{l i q}$. Die Lebensdauer $\tau_{l i q}$ wird als die Zeit definiert, die zwischen dem Zeitpunkt, zu dem die Kurve vom Maximum in das Plateau übergegangen ist, und dem Zeitpunkt, zu dem die Anzahl flüssiger Atome auf die Hälfte des durchschnittlichen Werts im Plateau $\left(N_{l i q}\right)$ zurückgegangen ist, liegt. Anfangs- und Endzeitpunkt des so definierten flüssigen Bereichs sind in Abb. 7.1 durch rote Linien markiert.

Die Darstellung der räumlichen Anordnung der flüssigen Atome zu einem Zeitpunkt im Bereich des Flüssigkeitsplateaus zeigt, dass sich im hier betrachteten metallischen Glas ein im Wesentlichen zusammenhängender, dichter, flüssiger Bereich bildet (siehe Abb. 7.1).

Beschüsse der CuTi Schichten sind bei einer Umgebungstemperatur von $T=10 \mathrm{~K}$ für verschiedene Energien bis einschließlich $E=1 \mathrm{keV}$ durchgeführt worden. Für diese Einschüsse sind jeweils die Flüssigkeitsanalysen durchgeführt und die daraus resultierenden Anzahlen flüssiger Atome und Lebensdauern der flüssigen Bereiche bestimmt worden (siehe Abb. 7.2). Bei der im Folgenden im Zentrum stehenden Energie von $E=1 \mathrm{keV}$ ergibt sich $N_{l i q}=1182 \pm 22$ und $\tau_{l i q}=2216 \pm 186$ fs.

Für die quantitative Bestimmung der Größe des flüssigen Bereichs werden jeweils zu einem Zeitpunkt im Flüssigkeitsplateau (siehe Abb. 7.1) die maximalen und minimalen Positionen flüssiger Atome in den verschiedenen Richtungen bestimmt. Die Resultate werden über mehrere Einschüsse gemittelt, so dass sich als Ergebnis für die Ausdehnung in z-Richtung $a_{z}=34 \pm 2 \AA$ bei $T=10 \mathrm{~K}$ Umgebungstemperatur ergibt.

Bei einer Umgebungstemperatur von $T=300 \mathrm{~K}$ gibt es bezüglich der Flüssigkeitsanalyse prinzipiell dieselben Phänomene wie bei einer Umgebungstemperatur von 
a.)

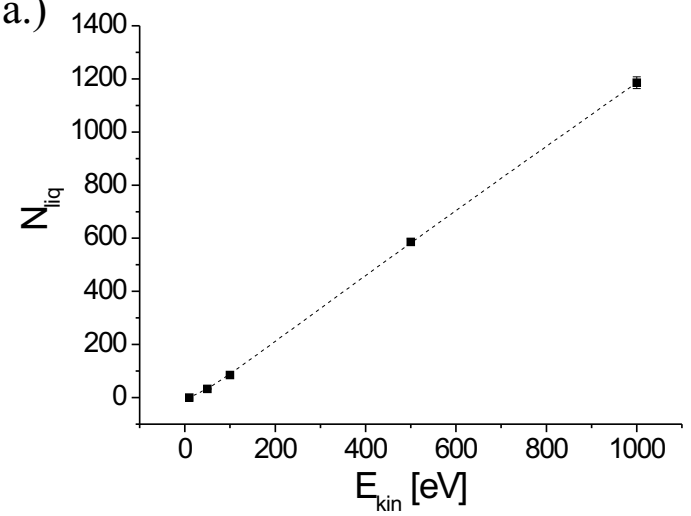

b.)

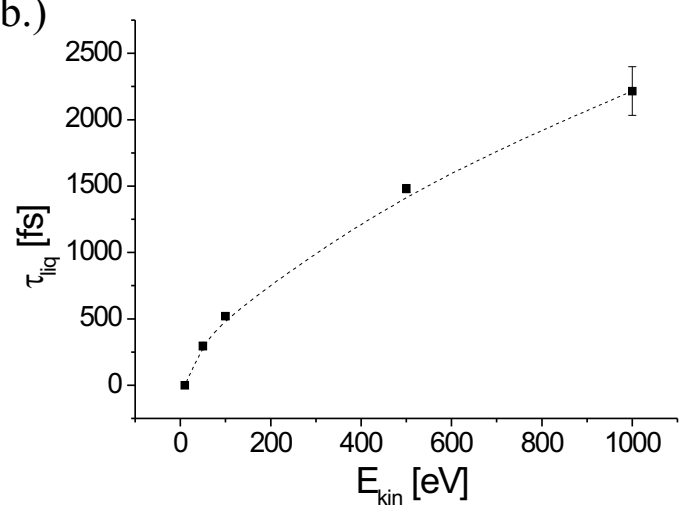

Abbildung 7.2: a.) Anzahl flüssiger Atome und b.) Lebensdauer flüssiger Bereiche jeweils als Funktion der Einschussenergie nach einem Einschuss eines $\mathrm{Cu}$ Atoms auf eine $\mathrm{CuTi}$ Schicht bei einer Umgebungstemperatur von $\mathrm{T}=10 \mathrm{~K}$. Gestrichelte Linien entsprechen einem Spline-Fit der Daten.

$T=10 \mathrm{~K}$. Erwartungsgemäß sind sowohl die Anzahl flüssiger Atome als auch die Lebensdauer der „thermal spikes“ größer (siehe Abb. 7.3). So ergeben sich beim Beschuss mit $\mathrm{Cu}$ Atomen der Energie $E=1 \mathrm{keV}$ als Werte $N_{\text {liq }}=2250 \pm 20, \tau_{\text {liq }}=5839 \pm 239 \mathrm{fs}$ und $a_{z}=42 \pm 2 \AA$.

\subsubsection{Analyse der atomaren Dynamik}

Methoden und Erwartungen Nachdem im vorangegangenen Abschnitt gezeigt worden ist, dass durch den Einschuss energiereicher $\mathrm{Cu}$ Atome auf eine $\mathrm{CuTi}$ Oberfläche flüssige Bereiche entstehen, wenn man eine einfache kinetische Definition benutzt, um ein Atom als flüssig zu bezeichnen, wird nun die atomare Dynamik in diesen Bereichen genauer untersucht. Damit soll geklärt werden, ob sich die Atome, die aufgrund von kinetischen Argumenten als flüssig bezeichnet werden, bezüglich ihrer Dynamik tatsächlich wie in einer Flüssigkeit verhalten.

Als quantitatives Maß zur Charakterisierung der atomaren Dynamik wird die Verteilung der atomaren Verschiebungsweiten, wie sie bereits in Kap. 6.1.3 definiert worden ist, verwendet. Auch für die hier untersuchten Einschüsse behält die theoretische Erwartung Gültigkeit, dass zusätzliche Maxima zum Hauptmaximum für die Anwesenheit einer Dynamik sprechen, bei der Einzelsprünge oder zumindest bevorzugt Sprünge mit der Sprungweite im Abstand von Nachbarn stattfinden, während ein Verlauf gemäß Gleichung (6.8) auf eine kooperative Dynamik schließen lässt. Um die Dynamik im zeitlich und räumlich begrenzten „thermal spike“ zu erfassen, wird ein hinreichend kleines Zeitintervall für die Analyse gewählt und es werden nur die Atome berücksichtigt, die gemäß der Definition flüssig sind. Um die Unterschiede zwischen bestrahlungsinduzierter und thermisch angeregter Dynamik sichtbar zu machen, wird bei dieser Untersuchung $T=10 \mathrm{~K}$ als Umgebungstemperatur verwendet, so dass thermisch angeregte Prozesse, 
a.)

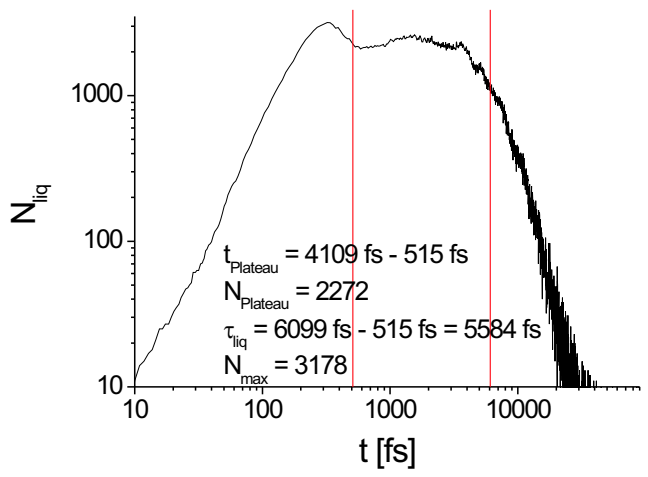

b.)

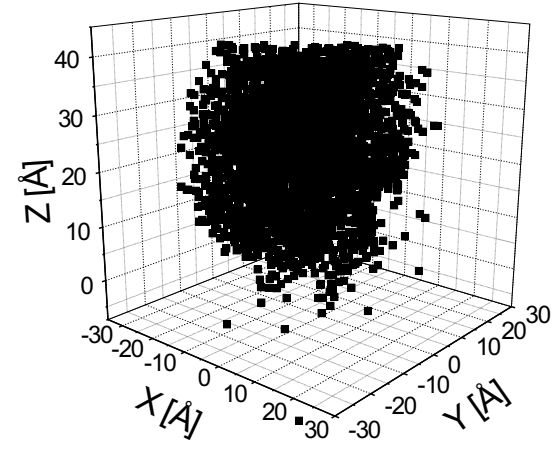

Abbildung 7.3: a.) Anzahl flüssiger Atome als Funktion der Zeit (Die roten Linien markieren zeitlich den Anfangs- und Endpunkt des flüssigen Bereichs.) und b.) räumliche Anordnung der flüssigen Atome zum Zeitpunkt $t=1501 \mathrm{fs}$, jeweils für einen $\mathrm{Cu}$ Einschuss der Energie $E=1 \mathrm{keV}$ auf $\mathrm{CuTi}$ bei $T=300 \mathrm{~K}$ Umgebungstemperatur.

deren Ursachen nicht mit dem Einschuss in Verbindung stehen, unterdrückt sind.

Ergebnisse In Abb. 7.4 ist die Verteilung der Verschiebungsweiten der $\mathrm{Cu}$ Atome aufgetragen, die sowohl zum „thermal spike“ als auch zum Oberflächenbereich der Probe nach Einschüssen von $\mathrm{Cu}$ Atomen der Energie $E=1 \mathrm{keV}$ gehören. Analysiert werden die Verschiebungsweiten zwischen den Zeiten $t_{1}=1$ ps und $t_{2}=2$ ps nach dem Einschuss, also innerhalb des Flüssigkeitsplateaus aus Abb. 7.1. Damit gehen ballistische Effekte, die sich vorher abspielen, nicht in die Analyse ein. Um statistisch aussagekräftige Resultate zu erreichen, wurde für diese Art der Analyse eine Vielzahl von Einschüssen simuliert. Die durchschnittlichen Wahrscheinlichkeiten für Verschiebungen über eine gegebene Distanz sind in Abb. 7.4 dargestellt. Mit eingetragen in den Graphen ist eine angepasste Funktion gemäß Gleichung (6.8). Diese einer radial gemittelten Gaußverteilung entsprechenden Kurve gibt die aus der Simulation gewonnenen Daten gut wieder. Es sind lediglich kleinere Abweichungen am rechten Ende des Hauptmaximums zu erkennen, aber an keiner Position deutet sich ein zweites Maximum an.

Besonders interessant ist der Vergleich der hier dargestellten Dynamik der Cu Atome im Bereich der Oberfläche nach dem Einschuss mit der Dynamik von $\mathrm{Cu}$ Atomen an der Oberfläche bei thermisch aktivierten Prozessen, wie sie in Kap. 6 untersucht wird. Deshalb sind in Abb. 7.5 die Verteilungen der Verschiebungsweiten für beide Arten der Anregung gemeinsam aufgetragen. Dort ist zu erkennen, dass bei thermischer Anregung bei $T=450 \mathrm{~K}$ und einer Beobachtungszeit $\Delta t=500$ ps für $\mathrm{Cu}$ Oberflächenatome ein zweites Maximum vorhanden ist und bei der strahlungsinduzierten Dynamik nicht. Des Weiteren fällt auf, dass die jeweils eingetragenen Fitkurven gemäß Gleichung (6.8) ihr Maximum für beide Arten der Anregung ziemlich genau an der gleichen Position haben. 


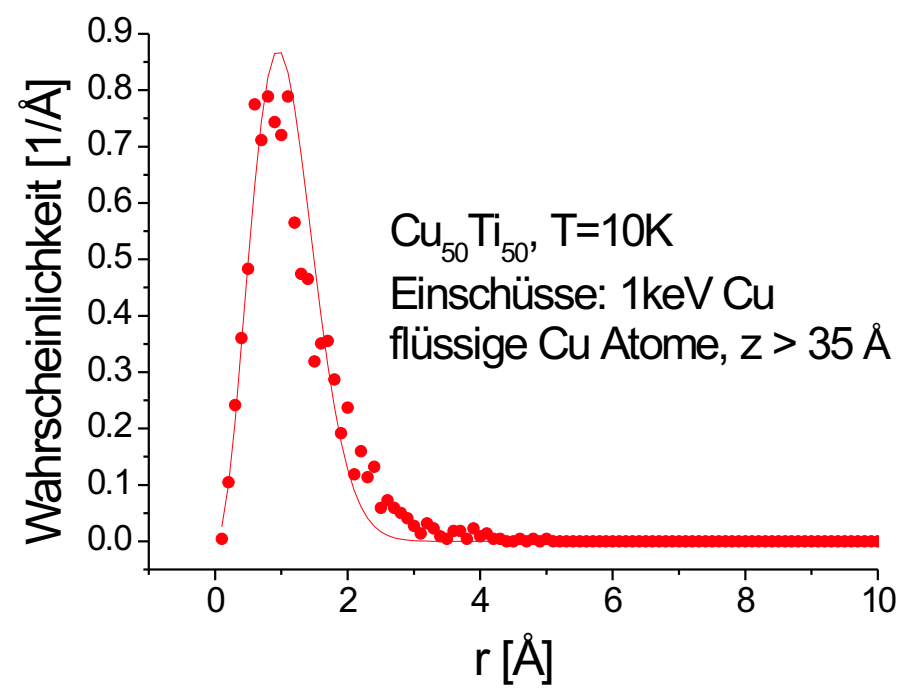

Abbildung 7.4: Verteilung der Verschiebungsweiten der flüssigen $\mathrm{Cu}$ Atome im Bereich der mit $\mathrm{Cu}$ Atomen der Energie $E=1 \mathrm{keV}$ beschossenen Oberfläche $(z>35 \AA)$ im Zeitraum $t_{1}=1$ ps bis $t_{2}=2$ ps bei einer Umgebungstemperatur von $T=10 \mathrm{~K}$ zusammen mit einem Fit entsprechend Gleichung (6.8).

Dieses Verhalten wird in Kap. 7.2.2 diskutiert.

\subsubsection{Identifikation der Parameter aus Kontinuumsmodellen}

In diesem Kapitel werden die in Kap. 4 besprochenen Prozesse bezüglich ihres Beitrags zur gesamten Entwicklung der Oberflächenstruktur analysiert. Dabei wird der Schwerpunkt auf glättenden Prozessen liegen, weil die unter sehr ähnlichen Bedingungen durchgeführten Experimente in Kap. 5 die klare Dominanz der glättenden Prozesse zeigen. Wie die Gewichte der einzelnen Prozesse miteinander quantitativ verglichen werden können, ist in Kap. 4.6 erläutert. Zunächst werden Ausdrücke bestimmt, die zwei Prozesse vergleichen. Ausgewertet werden die Ausdrücke zur Beschreibung der relativen Gewichte wiederum für Einschüsse der Energie $E=1 \mathrm{keV}$ bei Umgebungstemperaturen von $T=300 \mathrm{~K}$ und $T=10 \mathrm{~K}$.

\subsubsection{Physikalische Einheiten bei Bestrahlungsprozessen}

In der Literatur wird bei der Untersuchung von Bestrahlungsprozessen häufig die Größe der "displacements per atom" (dpa) verwendet, um die Fluenz zu ersetzen, weil weitere Größen wie die Ionensorte und die Bestrahlungsenergie allesamt in die Anzahl der Verschiebungen pro Atom (dpa) eingehen [AR98]. Deshalb erleichtert die Verwendung der Größe dpa den Vergleich von Bestrahlungsphänomenen bei verschiedenen Energien, 


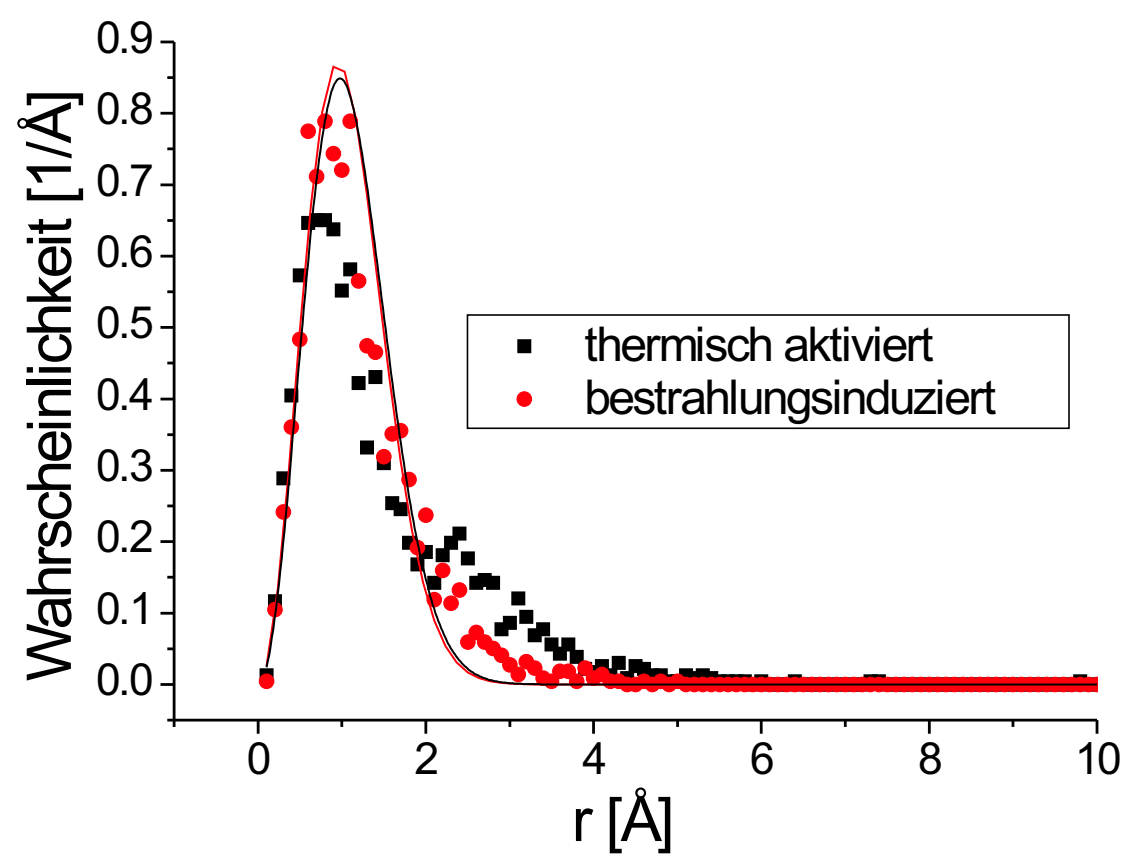

Abbildung 7.5: Verteilung der Verschiebungsweiten bei thermisch angeregter und bei strahlungsinduzierter Dynamik für $\mathrm{Cu}$ Atome im Oberflächenbereich aus Abb. 6.6.a bzw. Abb. 7.4 jeweils mit dem zugehörigen Fit gemäß Gleichung (6.8).

Ionensorten und Schichtmaterialien.

Bei der Untersuchung von dynamischen Prozessen, die durch Bestrahlungsereignisse induziert sind, ist es deshalb angebracht, die zur Fluenz proportionale Zeit durch die Anzahl der Verschiebungen pro Atom (dpa) zu ersetzen, wie es z.B. in [MAAA03] bei der Angabe von Viskositäten erfolgt ist. Dementsprechend werden in dieser Arbeit die Diffusionskonstanten für strahlungsinduzierte Prozesse in der Einheit $\mathrm{m}^{2} / \mathrm{dpa}$ angegeben.

Zur Berechnung der Anzahl von Verschiebungen pro Atom wird das Simulationsprogramm SRIM verwendet [ZB03]. Dieses Programm basiert auf auf einem Monte Carlo Modell, das im statistischen Mittel die physikalischen Größen im Rahmen der Näherung binärer Stöße (binary collision approximation: BCA), liefert. Die zu beschießende Schicht und die Sorte der eingeschossenen Atome sowie deren Energie können in SRIM vorgegeben werden und unter den gegebenen Bedingungen kann eine beliebige Zahl von Einschüssen simuliert werden. Eines der gelieferten Ergebnisse einer solchen Simulation ist die durchschnittliche Zahl von Verschiebungen (displacements) pro Längeneinheit und pro Ion $\sigma$ in der Einheit Verschiebungen/( $\AA$.Ion). Wenn man dieses Ergebnis mit dem in der MD-Simulation verwendeten Fluss $F$ und dem atomaren Volumen $\Omega$ multipliziert, ergibt sich daraus die Anzahl atomarer Verschiebungen pro Atom und Zeit, also 
die quantitative Beziehung zwischen den Einheiten dpa und s, so dass eine Umrechnung zwischen den entsprechenden Größen möglich ist:

$$
\begin{aligned}
\sigma \cdot F \cdot \Omega\left[\frac{\text { Verschiebungen }}{\AA \cdot \text { Ion }} \cdot \frac{\text { Ion }}{\AA^{2} \cdot \mathrm{s}} \cdot \frac{\AA^{3}}{\text { Atom }}\right] & =\sigma \cdot F \cdot \Omega\left[\frac{\text { Verschiebungen }}{\text { Atom }} \cdot \frac{1}{\mathrm{~s}}\right] \\
& =\sigma \cdot F \cdot \Omega\left[\frac{\mathrm{dpa}}{\mathrm{s}}\right] .
\end{aligned}
$$

\subsubsection{Viskoses Oberflächenfließen und Oberflächendiffusion}

Es gibt zwei glättende Terme mit derselben Abhängigkeit von der Oberflächenmorphologie, nämlich die für Oberflächendiffusion und viskoses Oberflächenfließen. Für das Verhältnis des Gewichts beider Prozesse $\Delta_{1}$ ergibt sich unter Verwendung der Gleichungen (4.22), (4.26) und (4.8)

$$
\Delta_{1}:=\frac{\left(b_{4} q^{4}\right)_{\text {Oberflächenfließen }}}{\left(b_{4} q^{4}\right)_{\text {Oberflächendiffusion }}}=\frac{\frac{\gamma a_{z}^{3}}{3 \eta} q^{4}}{\frac{D_{s} \gamma \Omega^{2} \nu}{k_{B} T} q^{4}}=\frac{a_{z}^{3} k_{B} T}{3 \eta D_{s} \Omega^{2} \nu}
$$

mit den in Kap. 4.5 definierten Größen. Es verbleibt also die Aufgabe, den Wert dieses Ausdrucks, der nicht abhängig von der Oberflächenmorphologie oder einer Wellenlänge ist, zu bestimmen. Das atomare Volumen $\Omega$ sowie die Oberflächendichte der zur Oberflächendiffusion beitragenden Atome $\nu$ ergeben sich direkt aus der Simulation. Die Dicke des flüssigen Bereichs $a_{z}$ ist bereits in Kap. 7.1.1 ausgewertet worden. Damit müssen noch die Viskosität $\eta$, die die Stärke des Oberflächenfließens beschreibt, und der Quotient $D_{s} / T$ bestimmt werden. Der Quotient $D_{s} / T$ ist ein Maß für die Beweglichkeit, die gemäß der Einstein-Relation durch $D_{s} /\left(k_{B} T\right)$ gegeben ist [KTH91], und beschreibt den Glättungsbeitrag der Oberflächendiffusion. Zunächst werden im Folgenden die Methoden beschrieben, mit denen eine solche Bestimmung möglich ist.

Die strahlungsinduzierte Viskosität wurde für das hier behandelte CuTi System bereits von S. G. Mayr mit Hilfe von MD-Simulationen berechnet [MAAA03]. Zur Bestimmung der Viskosität wird in der zitierten Arbeit während der Bestrahlung eine biaxiale mechanische Spannung angelegt und aus dem Verhältnis dieser mechanischen Spannung zur resultierenden Dehnung wird die Viskosität berechnet.

Zur Berechnung des Quotienten $D_{s} / T$ werden in dieser Arbeit drei verschiedene Verfahren verwendet. Allen Methoden gemein ist die Art der Simulation. Die wie in Kap. 3.4 beschrieben hergestellte Probe wird entlang der Substratnormalen mit einem Cu Atom beschossen. Solche Beschüsse werden an verschiedenen Orten der Probenoberfläche durchgeführt, um eine ausreichend gute Statistik der Ergebnisse zu gewährleisten. Die atomare Dynamik wird während der gesamten Zeit anhand der Orte der einzelnen Atome analysiert. Für sämtliche Analysen werden selbstgeschriebene IDL-Programme ${ }^{1}$ verwendet. Die Varianz der Atome wird zeitlich aufgelöst aufgenommen. Gemäß Gleichung (6.3) können damit die Diffusionskoeffizienten berechnet werden. An dieser Stelle ist diese allgemeinere Form der Gleichung nötig, weil der Einschuss eines Atoms auf die freistehende

\footnotetext{
${ }^{1}$ Interactive Data Language
} 


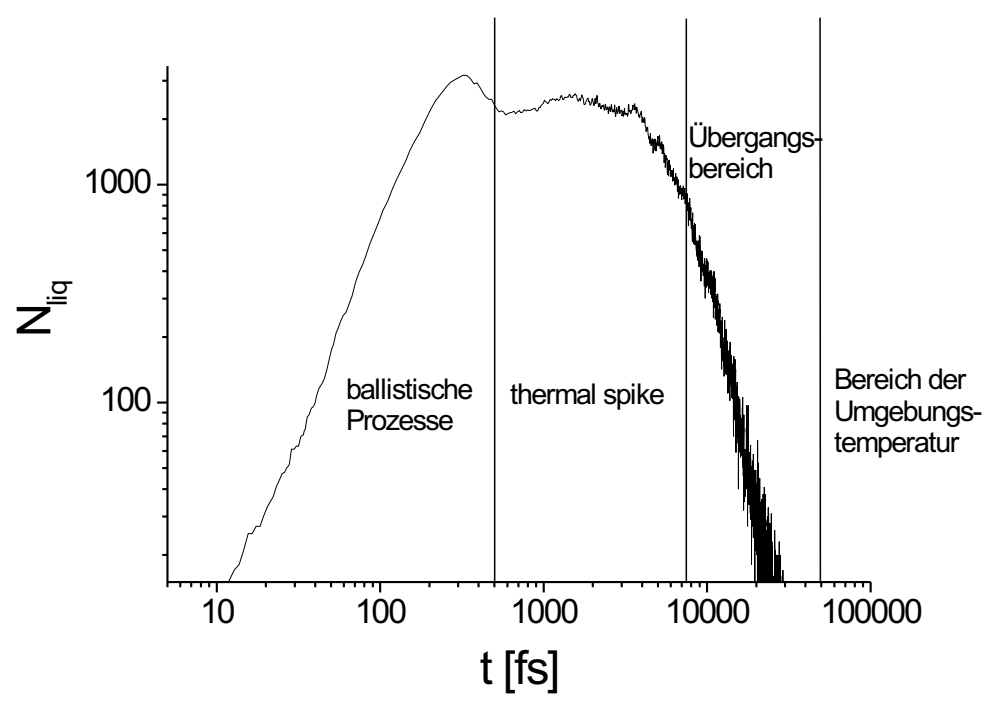

Abbildung 7.6: Anzahl flüssiger Atome als Funktion der Zeit für einen Cu Einschuss der Energie $E=1 \mathrm{keV}$ auf CuTi bei $T=300 \mathrm{~K}$ Umgebungstemperatur (aus Abb. 7.3) mit Kennzeichnung der verschiedenen Abschnitte.

dünne Schicht eine Gesamtbewegung der Probe bewirkt, die bei längeren Beobachtungszeiten in den atomaren Verschiebungen deutlich wird. Außerdem ist stets sichergestellt, dass bei der Berechnung von Diffusionskoeffizienten die Atome, die aufgrund des Beschusses die Probe verlassen, sowie das eingeschossene Atom selbst nicht berücksichtigt werden, da die Längen der Strecken, die außerhalb der Probe zurückgelegt werden, nicht mit einem Diffusionskoeffizienten verknüpft sind.

Methode 1 besteht darin, über den gesamtem simulierten Zeitraum die Varianz der Atome zu bestimmen und damit den Diffusionskoeffizienten zu berechnen. Um den durch den Beschuss beeinflussten Oberflächendiffusionskoeffizienten zu berechnen, werden nur die Atome berücksichtigt, die sich im Bereich der Oberfläche, auf der der Einschuss stattfindet, befinden. Wie bei den Untersuchungen zu thermisch aktivierten Prozessen in Kap. 6.1 werden die Atome als Oberflächenatome bezeichnet, die sich innerhalb der oberen $5 \AA$ der Probe befinden. Für $T$ wird die Umgebungstemperatur eingesetzt. Dadurch ergibt sich eine Abweichung von den realen Bedingungen, denn nach dem Beschuss liegt die Temperatur im Bereich des Einschusses 2 deutlich oberhalb der Umgebungstemperatur. Die tatsächliche Temperatur ist weder als Funktion der Zeit noch als Funktion des Ortes konstant. Trotzdem soll hier der konstante Wert der Umgebungstemperatur zunächst als erste Näherung verwendet werden. Weil diese Temperatur kleiner (oder gleich) im Vergleich zur tatsächlichen ist, ergibt sich daraus eine obere Grenze für das

${ }^{2}$ Da die Temperatur für Gleichgewichtszustände definiert ist, ist es frühestens nach dem Ende der ballistischen Prozesse, also mit Beginn des „thermal spikes“, sinnvoll, von einer Temperatur zu sprechen. 
Gewicht des Glättungsprozesses der Oberflächendiffusion.

Methode 2 besteht darin, die Analyse der atomaren Dynamik auf verschiedene Zeitbereiche aufzuteilen (siehe auch Diskussion in Kap. 7.2.1 zur Einordnung der verschiedenen Abschnitte). Wie bereits anhand der Analyse der Anzahl flüssiger Atome nach einem Einschuss in Kap. 7.1.1 dargestellt, dominieren zunächst ballistische Prozesse (Abschnitt 1). Anschließend bildet sich ein „thermal spike“ (Abschnitt 2). Nachdem dieser verschwindet, geht die Temperatur in einer Übergangsphase (Abschnitt 3) auf die Umgebungstemperatur zurück, um dort für den Rest der Simulation zu verharren (Abschnitt 4). Zur Verdeutlichung der Zuordnung sind die verschiedenen Abschnitte in die Darstellung der Anzahl flüssiger Atome als Funktion der Zeit in Abb. 7.6 eingetragen. Diese Aufteilung in verschiedene Abschnitte ermöglicht eine genauere Auswertung des Ausdrucks $D_{s} / T$. Die Gesamtzeit der Simulation pro Einschuss $t_{\text {ges }}$ entspricht der Summe der Zeiten der einzelnen Abschnitte $t_{i}$, d.h. $t_{g e s}=\sum_{i} t_{i}$. Es kann nun für jeden einzelnen Abschnitt $i$ der Ausdruck $D_{s, i} / T_{i}$ ausgewertet werden. Die Prozesse der einzelnen Abschnitte finden zeitlich nacheinander statt und sind statistisch unabhängig. Aufgrund der Struktur der Evolutionsgleichung (4.22) für Oberflächendiffusion ergibt sich der Gesamtausdruck gemäß

$$
D_{s} / T=\sum_{i} D_{s, i} / T_{i}
$$

wobei in der Berechnung der Diffusionskoeffizienten $D_{s, i}$ an dieser Stelle immer die Gesamtzeit der Simulation pro Einschuss eingesetzt werden muss. Diese Beziehung lässt sich verstehen, wenn man allein den deterministischen Glättungsmechanismus der Oberflächendiffusion betrachtet, für den sich nach Gleichung (4.9) und Gleichung (4.22) die Lösung

$$
\hat{h}\left(\vec{q}, t_{\text {ges }}\right)=\hat{h}_{0}(\vec{q}) \cdot \exp \left(-A q^{4} \frac{D_{s}}{T} t_{\text {ges }}\right)
$$

ergibt. Hier sind die konstanten Größen in $A=\frac{\gamma \Omega^{2} \nu}{k_{B}}$ zuammengefasst und $\hat{h}_{0}(\vec{q})=$ $\hat{h}(\vec{q}, t=0)$ beschreibt die Höhenfunktion zu Beginn der Simulation. Ersetzt man nun den gesamten Glättungsprozess durch die der zuvor festgelegten einzelnen Abschnitte, die zeitlich nacheinander erfolgen, so glättet der Prozess des ersten Abschnitts die ursprüngliche Struktur, die sich daraus ergebende Struktur wird durch den Prozess des zweiten Abschnitts geglättet, usw. Mathematisch formuliert liefert das die folgende erste 
Gleichung, die sich wie in den anschließenden Zeilen geschehen umformen lässt.

$$
\begin{aligned}
\hat{h}\left(\vec{q}, t_{\text {ges }}\right) & =\hat{h}_{0}(\vec{q}) \cdot \prod_{i} \exp \left(-A q^{4} \frac{\tilde{D}_{s, i}}{T_{i}} t_{i}\right) \\
& =\hat{h}_{0}(\vec{q}) \cdot \prod_{i} \exp \left(-A q^{4} \frac{\left\langle\left(\Delta \overrightarrow{r_{j}}-\left\langle\Delta \vec{r}_{j}\right\rangle_{s, i}\right)^{2}\right\rangle_{s, i}}{6 t_{i} T_{i}} t_{i}\right) \\
& =\hat{h}_{0}(\vec{q}) \cdot \prod_{i} \exp \left(-A q^{4} \frac{\left\langle\left(\Delta \overrightarrow{r_{j}}-\left\langle\Delta \overrightarrow{r_{j}}\right\rangle_{s, i}\right)^{2}\right\rangle_{s, i}}{6 t_{\text {ges }} T_{i}} t_{\text {ges }}\right) \\
& =\hat{h}_{0}(\vec{q}) \cdot \prod_{i} \exp \left(-A q^{4} \frac{D_{s, i}}{T_{i}} t_{\text {ges }}\right) \\
& =\hat{h}_{0}(\vec{q}) \cdot \exp \left(-A q^{4} \sum_{i} \frac{D_{s, i}}{T_{i}} t_{\text {ges }}\right) .
\end{aligned}
$$

Die Bedeutungen von $\tilde{D}_{s, i}$ und $D_{s, i}$ ergeben sich jeweils aus den benachbarten Zeilen und \langle\rangle$_{s, i}$ bedeutet die Mittelung über die durch $j$ indizierten Oberflächenatome während des Abschnitts $i$. Ein Vergleich der letzten Zeile in Gleichung (7.5) mit Gleichung (7.4) liefert die in Gleichung (7.3) angegebene Beziehung.

Die Werte für $D_{s, i}=\left\langle\left(\Delta \overrightarrow{r_{j}}-\left\langle\Delta \overrightarrow{r_{j}}\right\rangle_{s, i}\right)^{2}\right\rangle_{s, i} /\left(6 t_{\text {ges }}\right)$ werden direkt aus den Atompositionen zu den Zeitpunkten, an denen die Abschnitte wechseln, bestimmt. Weil ballistische Effekte nicht als Diffusionsprozesse angesehen werden können, wird der Beitrag aus dem ersten Abschnitt hier nicht mit einbezogen. Der Beitrag ballistischer Prozesse zur Glättung wird an späterer Stelle in dieser Arbeit noch untersucht (siehe Kap. 7.1.3.4). Während des Abschnitts des „thermal spike“ kann im Bereich der betrachteten Oberfläche die Glastemperatur für $T_{2}$ eingesetzt werden, denn in Kap. 7.1.1 hat sich gezeigt, dass diese Temperatur von vielen Atomen aus diesem Bereich überschritten ist. Im Zentrum des „thermal spike" liegt sie tatsächlich noch deutlich darüber, während sie in den Randbereichen der Oberfläche darunter liegt. Während der Übergangsphase der Probentemperatur zur Umgebungstemperatur und der anschließenden Phase des Verbleibens bei dieser Temperatur (Abschnitte 3,4) wird die Umgebungstemperatur für $T_{3}, T_{4}$ eingesetzt. Somit wird auch bei dieser Methode ein unterer Grenzwert für die benötigten Temperaturen verwendet, so dass sich für das Gewicht des Glättens durch Oberflächendiffusion ebenfalls ein oberer Grenzwert ergibt.

Methode 3 wird benötigt, weil die MD-Simulationen bezüglich des erfassbaren Zeitintervalls limitiert sind. Diese Begrenzung kann bei Umgebungstemperaturen, bei denen thermisch aktivierte Diffusion eine Rolle spielt, bewirken, dass Prozesse, die auf längeren Zeitskalen, als sie hier simuliert werden, stattfinden, nicht erfasst werden und somit der 
Diffusionskoeffizient als zu klein berechnet wird. Diese Unzulänglichkeit ist bei Umgebungstemperaturen von $T=300 \mathrm{~K}$ relevant, aber nicht bei $T=10 \mathrm{~K}$, wo keine thermisch aktivierte Diffusion mehr stattfindet [MT94; $\left.\mathrm{BEH}^{+} 01\right]$. Während in Methode 1 und 2 nur die Zeitskala der MD-Simulation (1 ns) erfasst wird, soll mit Methode 3 eine experimentell relevante Zeitskala erfasst werden.

Für $T=300 \mathrm{~K}$ soll deshalb von Beginn des Abschnitts 4 (vgl. Abb. 7.6) an die Diffusion auf eine andere Art berechnet werden. Von metallischen Gläsern ist bekannt, dass weit unterhalb der Glastemperatur Oberflächendiffusion nur dann eine messbare Rolle spielt, wenn weniger stark gebundene Atome, so genannte Adatome, vorhanden sind. Solche Adatome existieren z.B. während des Wachstums aufgedampfter dünner Schichten $\left[\mathrm{RML}^{+} 00\right]$. In der zitierten Arbeit wird auch eine durchschnittliche Diffusionslänge solcher Adatome angegeben mit $s=1 \mathrm{~nm}$ für $\mathrm{Zr}_{65} \mathrm{Al}_{7.5} \mathrm{Cu}_{27.5}$. Diese Diffusionslänge ist die auf experimenteller Zeitskala relevante. Es wird angenommen, dass ein Adatom im Mittel diesen Weg zurücklegt, bis es eine stärkere Bindung eingeht und somit kein Adatom mehr ist. Durch atomare Einschüsse werden ebenfalls Adatome erzeugt [AR98; $\left.\mathrm{BEH}^{+} 01\right]$. Wenn man annimmt, dass im hier simulierten $\mathrm{Cu}_{50} \mathrm{Ti}_{50}$ die Verhältnisse ähnlich wie im $\mathrm{Zr}_{65} \mathrm{Al}_{7.5} \mathrm{Cu}_{27.5}$ sind (siehe Kap. 3.3.2 zur Begründung), so kann mit Hilfe der durchschnittlichen Diffusionslänge ein Koeffizient für die durch Adatome induzierte Oberflächendiffusion bestimmt werden, wenn die Anzahl der pro eingeschossenem Ion produzierten Adatome bekannt ist. Für die Oberflächendiffusion gilt mit der Anzahl von Adatomen $N_{\text {Adatom }}$ und der Anzahl von Oberflächenatomen $N_{s}$

$$
D_{s}=\frac{s^{2}}{4 t} \frac{N_{\text {Adatom }}}{N_{s}}
$$

Im Nenner erscheint hier der Faktor 4, weil Adatomdiffusion per Definition 2-dimensional auf der Oberfläche stattfindet, während bei den zuvor betrachteten Diffusionskoeffizienten immer Bewegungen in allen drei Raumrichtungen berücksichtigt wurden, so dass dort immer der Faktor 6 erscheint. Die Zeit wird auch in dieser Formel in die für strahlungsinduzierte Prozesse relevante Einheit dpa umgerechnet; die Anzahl der pro Einschuss erzeugten Defekte wird wie in Kap. 7.1.3.1 beschrieben abgeschätzt. Die Anzahl der Oberflächenatome auf der Seite, die beschossen wird, steht für eine würfelförmige Simulationszelle mit der Gesamtzahl von Atomen $N$ über $N_{s}=N^{2 / 3}$ in Beziehung. Somit verbleibt noch die Bestimmung der Anzahl von Adatomen. Die entsprechenden Methoden werden im Folgenden erläutert.

Eine Methode, die Anzahl von Adatomen zu bestimmen, beruht auf einem geometrischen Kriterium. Wenn vor dem Einschuss die Oberfläche glatt ist, werden die Atome, die sich nach dem Einschuss oberhalb der ursprünglichen Oberfläche befinden, als Adatome bezeichnet $\left[\mathrm{BEH}^{+} 01\right]$. In der zitierten Arbeit werden genauer gesagt nur die Atome als Adatome gezählt, die sich mit einem geforderten Mindestabstand über der ursprünglichen Oberfläche befinden. Eine solche Definition ist für kristalline Oberflächen besonders gut geeignet, weil sich dort bezogen auf die glatte Oberfläche geometrisch einfach klären lässt, ob ein Atom mehr oder weniger stark gebunden ist und weil die glatte ursprüngliche Oberfläche gut definiert ist. Bei einer amorphen Oberfläche ist die 
Situation komplexer, weil die Oberflächen durch eine statistische atomare Rauigkeit gekennzeichnet sind, aber auch hier wird beobachtet (siehe nachfolgende Ergebnisse), dass Atome über die ursprüngliche Oberfläche angehoben werden. Diese Atome werden im Folgenden als Adatome bezeichnet, sofern sie die Oberfläche nicht verlassen und somit als gesputterte Atome gewertet werden.

Dass sich die in der Simulation verwendeten freistehenden Schichten durch den Impulsübertrag des eingeschossenen Atoms bewegen können, muss in die Definition der als Referenz geltenden ursprünglichen Oberfläche bedacht werden. Die Position der unteren Grenze der Schicht wird aus der durchschnittlichen $z$-Position der $N_{\text {min }}$ Atome mit den kleinsten z-Koordinaten zu $\langle z\rangle_{\min }$ bestimmt. Die Position einer virtuellen Oberfläche lässt sich dann mit einer konstanten Länge $d_{z}$ durch $z_{s}=\langle z\rangle_{\min }+d_{z}$ bestimmen. Oberhalb der durch $z_{s}$ markierten virtuellen Oberfläche befinden sich $\tilde{N}$ Atome. Dabei werden das eingeschossene sowie gesputterte Atome nicht mitgezählt. Die Werte von $N_{\min }$ und $d_{z}$ sollten so gewählt sein, dass $\tilde{N}$ für die ursprüngliche Oberfläche im Bereich der Anzahl von Oberflächenatomen ist. Dann lässt sich während der Simulation eines Einschusses die Anzahl der durch den Einschuss erzeugten Adatome gemäß $N_{\text {Adatom }}(t)=\tilde{N}(t)-\tilde{N}(t=0)$ zeitabhängig bestimmen. Durch Simulation mehrerer Einschüsse an verschiedenen Orten auf der Oberfläche kann der typische Verlauf der Anzahl so definierter Adatome als Funktion der Zeit ermittelt werden.

Die zweite Methode zur Bestimmung der Anzahl von Adatomen beruht auf einer Analyse der Energieverhältnisse. Wie bereits erwähnt zeichnen sich Adatome dadurch aus, dass sie schwächer gebunden sind als andere Oberflächenatome. Eine Analyse der potentiellen Energie in den Proben vor einem Einschuss wurde bereits in Kap. 6 besprochen, allerdings für höhere Temperaturen als $T=300 \mathrm{~K}$. Es ergeben sich aber auch für $T=300 \mathrm{~K}$ qualitativ gleiche Ergebnisse. Danach gibt es insbesondere für $\mathrm{Cu}$ energetisch höher liegende Atome im Oberflächenbereich, die mögliche Adatome darstellen. Deshalb können als durch einen Einschuss produzierte Adatome solche Atome gezählt werden, deren potentielle Energie nach dem Einschuss die Grenze $E_{M}+2 \sigma_{E}\left(E_{M}\right.$ und $\sigma_{E}$ sind Mittelwert und Standardabweichung der angepassten Gaußverteilung, siehe Kap. 6.1.4) überschreitet und vor dem Einschuss darunter liegt, und die sich außerdem innerhalb der oberen $5 \AA$ der Probe befinden. Dieser Methode entsprechend können solche Atome aus dem Oberflächenbereich, die vor dem Einschuss oberhalb der Energiegrenze und nach dem Einschuss darunter liegen, als vernichtete Adatome gezählt werden, so dass sich aus der Differenz von erzeugten und vernichteten Adatomen der Nettozuwachs pro Einschuss ergibt.

\subsubsection{Ergebnisse zum Vergleich von viskosem Oberflächenfließen und Oberflächendiffusion}

Die Werte für strahlungsinduzierte Fluiditäten $H$, die dem Inversen der Viskositäten entsprechen, sind von S. G. Mayr für das hier verwendete $\mathrm{CuTi}$ bereits berechnet worden [MAAA03], und zwar mit den Ergebnissen $H(10 \mathrm{~K})=3 \cdot 10^{-9}(\mathrm{~Pa} \cdot \mathrm{dpa})^{-1}$, $H(10 \mathrm{~K})=6 \cdot 10^{-9}(\mathrm{~Pa} \cdot \mathrm{dpa})^{-1}$ und $H(10 \mathrm{~K})=9 \cdot 10^{-9}(\mathrm{~Pa} \cdot \mathrm{dpa})^{-1}$. Interpolation zwischen diesen Fluiditäten liefert für die Viskosität bei Raumtemperatur 


\begin{tabular}{|l|l|l|l|l|}
\hline & $\eta[\mathrm{Pa} \cdot \mathrm{dpa}]$ & $a_{z}[\AA]$ & $\Omega\left[\AA^{3}\right]$ & $\nu\left[\AA^{-2}\right]$ \\
\hline$T=300 \mathrm{~K}$ & $1.25 \cdot 10^{8}$ & $42 \pm 2$ & 14.66 & 0.167 \\
\hline$T=10 \mathrm{~K}$ & $3.33 \cdot 10^{8}$ & $34 \pm 2$ & 14.41 & 0.169 \\
\hline
\end{tabular}

Tabelle 7.1: Viskosität, vertikale Ausdehnung des flüssigen Bereichs, mittleres atomares Volumen und atomare Oberflächendichte während der Bestrahlung von CuTi Proben.

$\eta(300 \mathrm{~K})=1.25 \cdot 10^{8} \mathrm{~Pa} \cdot \mathrm{dpa}$ und für $T=10 \mathrm{~K}$ gilt $\eta(10 \mathrm{~K})=3.33 \cdot 10^{8} \mathrm{~Pa} \cdot \mathrm{dpa}$.

Wie in Kap. 7.1.3.1 beschrieben erfolgt die Umrechnung zwischen den Einheiten s und dpa mit Hilfe der Ergebnisse aus SRIM Simulationen. Im Bereich der oberen $5 \AA$ der Probe, die jeweils für die Auswertung der Oberflächendiffusion berücksichtigt werden, ergibt sich für $T=10 \mathrm{~K}$ bei einer Simulationszeit $t_{\text {ges }}$ pro Einschuss der Umrechnungsfaktor $2.25 \cdot 10^{-3} \frac{\mathrm{dpa}}{t_{\text {ges }}}$ und für $T=300 \mathrm{~K}$ der Umrechnungsfaktor $2.28 \cdot 10^{-3} \frac{\mathrm{dpa}}{t_{\text {ges }}}$. Die sehr kleine Temperaturabhängigkeit beruht hier nur auf den unterschiedlichen temperaturabhängigen atomaren Dichten in der MD-Simulation.

Die Ausdehnung des flüssigen Bereichs in z-Richtung sowie das mittlere atomare Volumen und die mittlere Dichte von Oberflächenatomen sind zusammen mit den Viskositäten in Tab. 7.1 angegeben.

\begin{tabular}{|l|l|l|l|l|}
\hline & $D_{s}^{C u} / T\left[10^{-19} \frac{\mathrm{m}^{2}}{\mathrm{dpa} \cdot \mathrm{K}}\right]$ & $D_{s}^{T i} / T\left[10^{-19} \frac{\mathrm{m}^{2}}{\mathrm{dpa} \cdot \mathrm{K}}\right]$ & $D_{s} / T\left[10^{-19} \frac{\mathrm{m}^{2}}{\mathrm{dpa} \cdot \mathrm{K}}\right]$ & $\boldsymbol{\Delta}_{\mathbf{1}}$ \\
\hline $\begin{array}{l}T=300 \mathrm{~K} \\
\Delta t=1 \mathrm{~ns}\end{array}$ & $(12.6 \pm 1.8) / 300$ & $(9.9 \pm 1.4) / 300$ & $(11.3 \pm 2.3) / 300$ & $\mathbf{2 0 2} \pm \mathbf{5 0}$ \\
\hline $\begin{array}{l}T=300 \mathrm{~K} \\
\Delta t=50 \mathrm{ps}\end{array}$ & $(10.6 \pm 1.6) / 300$ & $(8.4 \pm 1.2) / 300$ & $(9.5 \pm 2.0) / 300$ & $\mathbf{2 4 3} \pm \mathbf{7 1}$ \\
\hline $\begin{array}{l}T=10 \mathrm{~K} \\
\Delta t=50 \mathrm{ps}\end{array}$ & $(7.5 \pm 1.0) / 10$ & $(5.6 \pm 0.7) / 10$ & $(6.5 \pm 1.2) / 10$ & $\mathbf{2 . 4} \pm \mathbf{0 . 7}$ \\
\hline
\end{tabular}

Tabelle 7.2: Gemäß Methode 1 bestimmte Diffusionskoeffizienten und resultierendes relatives Gewicht von viskosem Oberflächenfließen im Verhältnis zu Oberflächendiffusion bei verschiedenen Temperaturen und Simulationszeiten.

Die Quotienten aus Diffusionskoeffizienten und der Temperatur sind für Methode 1 und beide Umgebungstemperaturen in Tab. 7.2 eingetragen. Dort ist außerdem das zusammen mit den Daten aus Tab. 7.1 resultierende Ergebnis für $\Delta_{1}$, das sich bei der Verwendung des über die Elemente gemittelten Diffusionskoeffizienten ergibt, angegeben. Die Simulationen der Einschüsse sind bei $\mathrm{T}=10 \mathrm{~K}$ über einen Zeitraum von $\Delta t=50 \mathrm{ps}$ durchgeführt worden. Nach dieser Zeit sind die Effekte des Einschusses abgeklungen und die Temperatur ist so niedrig, dass keine Bewegung mehr stattfindet (siehe hierzu auch Kap. 7.1.1). Weil bei Raumtemperatur Bewegungen auch auf längeren Zeitskalen auftreten könnten, sind bei $T=300 \mathrm{~K}$ die Simulationen jeweils für $\Delta t=1 \mathrm{~ns}$ durchgeführt und sowohl für diese Zeit als auch für $\Delta t=50 \mathrm{ps}$ ausgewertet worden. Am Beispiel eines repräsentativen Einschusses ist der zeitliche Verlauf der Varianz in Abb. 7.7 dargestellt. Es zeigt sich, dass der wesentliche Beitrag zur Varianz direkt nach dem Einschuss erfolgt 


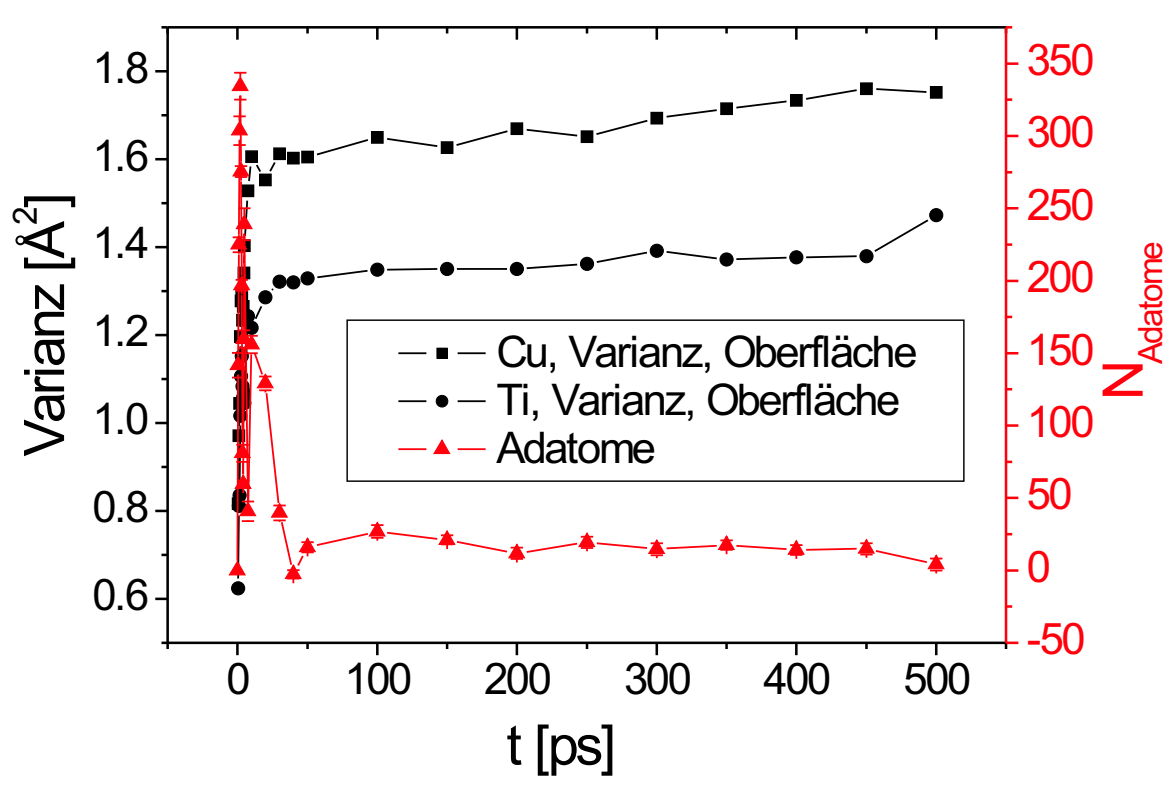

Abbildung 7.7: Varianz der $\mathrm{Cu}$ und Ti Atome im Oberflächenbereich nach Einschuss eines $\mathrm{Cu}$ Atoms der Energie $E=1 \mathrm{keV}$ und Anzahl der Adatome nach geometrischem Kriterium als Mittelung über mehrere Einschüsse jeweils bei einer Umgebungstemperatur von $T=300 \mathrm{~K}$.

(0 bis 10 ps). Daran schließt sich ein deutlich kleinerer Anstieg als Funktion der Zeit an.

Die entsprechenden Ergebnisse für die Auswertungen gemäß Methode 2 sind in Tab. 7.3 zu finden. Dabei sind die Zeitintervalle für $T=300 \mathrm{~K}$ Umgebungstemperatur in Übereinstimmung mit Abb. 7.6 so festgelegt, dass das ballistische Regime von 0 ps bis 0.5 ps, der thermal spike von 0.5 ps bis 7.5 ps, der Übergangsbereich von 7.5 ps bis 50 ps und der Bereich der Umgebungstemperatur von 50 ps bis 1000 ps festgelegt sind. Bei $T=10 \mathrm{~K}$ Umgebungstemperatur wird analog verfahren mit dem Unterschied, dass der letzte Abschnitt (50 ps bis $1000 \mathrm{ps}$ ) nicht berücksichtigt werden muss, weil bei $T=10 \mathrm{~K}$ keine thermisch aktivierte Diffusion stattfindet.

Im Rahmen von Methode 3 ist die zeitliche Entwicklung der über das zuvor beschriebene geometrische Kriterium bestimmten Adatome als Mittelung über mehrere Einschüsse in Abb. 7.7 zusammen mit der Entwicklung der Varianz eines typischen Einschusses dargestellt. Für die Anzahl von Adatomen ergibt sich direkt nach dem Einschuss ein deutliches Maximum, das aber nach weniger als 50 ps wieder zerfallen ist. Gesucht sind gerade solche Adatome, die auf langen Zeitskalen zur Diffusion beitragen. Deshalb wird die Anzahl von Adatomen als Mittelwert aus dem Bereich zwischen $t=50$ ps und $t=500 \mathrm{ps}$ bestimmt und dafür ergibt sich $N_{\text {Adatom }}=17 \pm 3$. Weil die durchschnittliche Diffusionslänge $s$ für Adatome sich aus einer Mittelung über die Elemente ergibt, kann hier ebenfalls nur ein gemittelter Wert angegeben werden und der lautet 


\begin{tabular}{|l|l|l|l|l|}
\hline & $D_{s}^{C u} / T\left[10^{-19} \frac{\mathrm{m}^{2}}{\mathrm{dpa} \cdot \mathrm{K}}\right]$ & $D_{s}^{T i} / T\left[10^{-19} \frac{\mathrm{m}^{2}}{\mathrm{dpa} \cdot \mathrm{K}}\right]$ & $D_{s} / T\left[10^{-19} \frac{\mathrm{m}^{2}}{\mathrm{dpa} \cdot \mathrm{K}}\right]$ & $\boldsymbol{\Delta}_{\mathbf{1}}$ \\
\hline $\begin{array}{l}T=300 \mathrm{~K} \\
\Delta t=1 \mathrm{~ns}\end{array}$ & $(11.8 \pm 0.8) / 300$ & $(9.8 \pm 0.7) / 300$ & $(10.8 \pm 1.1) / 300$ & $\mathbf{2 1 5} \pm \mathbf{3 7}$ \\
\hline $\begin{array}{l}T=10 \mathrm{~K} \\
\Delta t=50 \mathrm{ps}\end{array}$ & $(1.56 \pm 0.17) / 10$ & $(1.31 \pm 0.13) / 10$ & $(1.44 \pm 0.21) / 10$ & $\mathbf{1 0 . 9} \pm \mathbf{3 . 2}$ \\
\hline
\end{tabular}

Tabelle 7.3: Gemäß Methode 2 bestimmte Diffusionskoeffizienten und resultierendes relatives Gewicht von viskosem Oberflächenfließen im Verhältnis zu Oberflächendiffusion bei verschiedenen Temperaturen.

unter Verwendung von Gleichung (7.3) mit den eingesetzten Größen für Adatomdiffusion gemäß Gleichung (7.6) im Zeitintervall des Bereichs der Umgebungstemperatur $D_{s} / T=(25.1 \pm 2.3) \cdot 10^{-19} / 300 \frac{\mathrm{m}^{2}}{\mathrm{dpa} \cdot \mathrm{K}}$. Daraus ergibt sich für das relative Gewicht des viskosen Oberflächenfließens gegenüber dem der Oberflächendiffusion $\Delta_{1}=91 \pm 17$.

Die alternative Abschätzung der Anzahl von Adatomen über das beschriebene Energiekriterium liefert vergleichbare Ergebnisse. Demnach werden pro Einschuss im Mittel $14 \pm 1$ Ti Adatome und $37 \pm 3 \mathrm{Cu}$ Adatome, also insgesamt $51 \pm 4$ Adatome erzeugt. Zählt man mit dem beschriebenen Kriterium ebenfalls die als vernichtet geltenden Atome, so ergeben sich im Mittel sogar $46 \pm 3$ vernichtete $\mathrm{Cu}$ Adatome, $12 \pm 1$ vernichtete Ti Adatome und damit in der Summe $58 \pm 4$ vernichtete Adatome. Nach diesem Kriterium werden also durch den Einschuss nicht signifikant mehr Adatome erzeugt als vernichtet. Die zugehörigen Energieverteilungen vor und nach einem Einschuss sind für ein repräsentatives Beispiel in Abb. 7.8 dargestellt. Dort ist zu erkennen, dass sich die Energieverteilung nicht wesentlich ändert, insbesondere auch nicht in dem Energiebereich, für den ein Atom als Adatom in Betracht gezogen wird. Es entsteht also keine Verteilung, in der höhere Energien vertreten sind als vor dem Einschuss. Außerdem ist nicht zu beobachten, dass der Bereich der höchsten vorkommenden Energien signifikant stärker vertreten ist als vor dem Einschuss. Für eine obere Abschätzung der Anzahl von Adatomen kann die Anzahl der erzeugten Adatome verwendet werden. Sie liegt etwa bei dem dreifachen Wert des aus dem geometrischen Kriterium bestimmten Wertes. Damit würde sich für den entsprechenden Wert des Verhältnisses von viskosem Oberflächenfließen zur Oberflächendiffusion $\Delta_{1}=39 \pm 7$, also ebenfalls ein Wert, der deutlich größer als eins ist, ergeben.

Insgesamt führen alle Auswertungsmethoden zu dem Ergebnis, dass der Effekt des viskosen Oberflächenfließens den der Oberflächendiffusion überwiegt. Bei $\mathrm{T}=300 \mathrm{~K}$ liefern die Methoden 1 und 2, in denen die Diffusion direkt aus den atomaren Koordinaten der MD-Simulation und somit auf den der MD-Simulation zugänglichen Zeitskalen berechnet wird, eine deutlichere Dominanz des viskosen Oberflächenfließens als Methode 3 , bei der die Adatomdiffusion auf längeren Zeitskalen berücksichtigt wird. Zwischen den Methoden 1 und 2 besteht bei $T=300 \mathrm{~K}$ kein wesentlicher Unterschied. Bei $\mathrm{T}=10 \mathrm{~K}$ liefert Methode 2 eine stärkere Dominanz des viskosen Oberflächenfließens als Methode 1. Außerdem fällt auf, dass die Ergebnisse hier bei der tieferen Temperatur eine schwächere Dominanz des viskosen Oberflächenfließens besagen als die Ergebnisse bei 

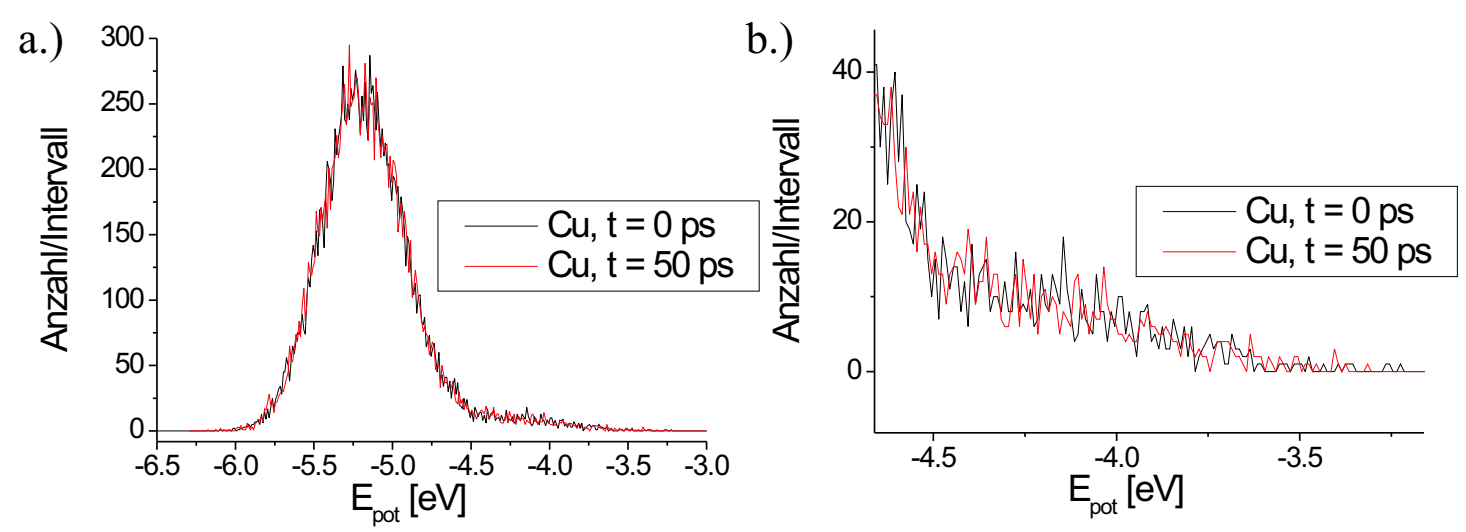

Abbildung 7.8: Verteilung der potentiellen Energie der $\mathrm{Cu}$ Atome vor $(t=0 \mathrm{ps})$ und nach $(t=50 \mathrm{ps})$ dem Einschuss eines $\mathrm{Cu}$ Atoms der Energie $E=1 \mathrm{keV}$. Teil a.) zeigt den gesamten Energiebereich und Teil b.) den Ausschnitt der Energie, für den Adatome in Betracht kommen.

der höheren Temperatur. Diese Abhängigkeit der Ergebnisse von der Analysemethode und der umgebenden Temperatur wird in Kap. 7.2.3 erläutert und diskutiert.

Aus den für Methode 1 und 2 berechneten Diffusionskoeffizienten der einzelnen Teilchensorten geht hervor, dass die $\mathrm{Cu}$ Atome unter allen untersuchten Bedingungen einen größeren Diffusionskoeffizienten als die Ti Atome aufweisen.

Insgesamt zeigen die dargestellten Ergebnisse, dass das viskose Oberflächenfließen die Oberflächendiffusion dominiert und das gilt unabhängig von der Wellenlänge der vorhandenen Strukturen.

\subsubsection{Viskoses Oberflächenfließen und ballistischer Transport}

Für das Verhältnis des Gewichtes dieser beiden Prozesse bezüglich der Glättung von Strukturen durch Bestrahlung ergibt sich mit Hilfe der Gleichungen (4.24) und (4.25):

$$
\Delta_{2}:=\frac{\left(b_{4} q^{4}\right)_{\text {Oberflächenfließen }}}{\left(b_{2} q^{2}\right)_{\text {ballistischer Transport }}}=\frac{\frac{\gamma a_{z}^{3}}{3 \eta} q^{4}}{F \Omega\left|\frac{\delta}{\tan (\alpha)}\right| q^{2}}=\frac{\gamma a_{z}^{3}}{3 \eta F \Omega}\left|\frac{\tan (\alpha)}{\delta}\right| q^{2} .
$$

Dieser Ausdruck ist abhängig von der Wellenlänge der Struktur, deren Glättung untersucht werden soll. Für Strukturen mit sehr großer Wellenlänge $\lambda=2 \pi / q$ dominiert der ballistische Transport, für kleine Wellenlängen das viskose Oberflächenfließen. Die Wellenlänge $\lambda_{c}$, an der der Übergang zwischen den beiden Bereichen stattfindet, hat genau den Wert, für den sich $\Delta_{2}=1$ ergibt. Das gilt für

$$
\lambda_{c}=2 \pi \sqrt{\frac{\gamma a_{z}^{3}}{3 \eta F \Omega}\left|\frac{\tan (\alpha)}{\delta}\right|} .
$$


Simulation

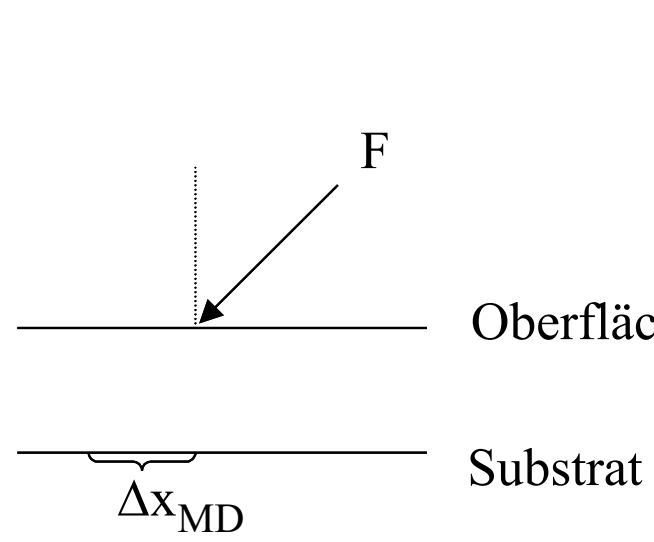

real

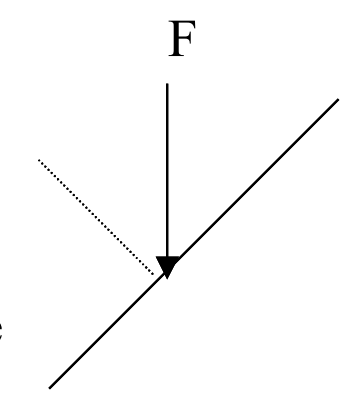

Abbildung 7.9: Schräger Beschuss in der Simulation und Beschuss von Hügelflanken im realen Fall.

Die Größen in diesem Ausdruck müssen nun aus entsprechenden MD-Simulationen bestimmt werden, um zu einem Ergebnis für die Übergangswellenlänge zu gelangen. Die Tiefe des flüssigen Bereiches $a_{z}$ sowie das atomare Volumen $\Omega$ und die Viskosität $\eta$ sind bereits in Tab. 7.1 aufgeführt. Der Fluss ist bekannt, da die Oberfläche der Simulationszelle und die Simulationszeit pro Einschuss bekannt sind. Entsprechend Kap. 7.1.3.1 wird die Zeit in dpa umgerechnet. Die freie Oberflächenenergie $\gamma$ soll in erster Näherung durch die Oberflächenenergie $\tilde{\gamma}$ ersetzt werden, die sich aus der Energiedifferenz einer Simulationszelle mit Oberflächen und einer Simulationszelle ohne Oberflächen berechnen lässt. Die Simulationszellen, die dünne Schichten beschreiben, haben zwei Oberflächen jeweils von der Größe $A$. Daher ergibt sich die Oberflächenenergie über

$$
\tilde{\gamma}=\frac{E_{\text {Schicht }}-E_{\text {Bulk }}}{2 A}
$$

Als wesentliche Aufgabe verbleibt nun, die Summe der lateralen atomaren Verschiebungen $\delta$ für verschiedene Winkel $\alpha$ zu bestimmen. Wie in Abb. 4.2 skizziert beschreibt $\alpha$ die Steigung der Hügelflanken, auf die das eingeschossene Atom trifft $(\tan (\alpha)=|\nabla h|)$. In der MD-Simulation wird diese Situation dadurch dargestellt, dass auf eine unverkippte Oberfläche unter einem Winkel $\alpha$ zur Oberflächennormalen die Atome eingeschossen werden. Beide Situationen unterscheiden sich lediglich durch eine Verkippung des Koordinatensystems (siehe Abb. 7.9). Zwischen dem der Realität entsprechenden System und dem aus der MD-Simulation ergeben sich für den Fluss und die Summe der atomaren Verschiebungen in der Substratebene die Beziehungen $F_{\mathrm{Re}}=F_{\mathrm{MD}} / \cos (\alpha)$ und $\delta_{\mathrm{Re}}=\delta_{\mathrm{MD}} \cos (\alpha)$. Daher ist das in den Gleichungen (7.7) und (7.8) relevante Produkt $F \cdot \delta$ unabhängig von der Wahl des Bezugssystems. Im weiteren Verlauf soll $\delta$ für die vorgestellten Ergebnisse $\delta_{M D}$ bedeuten, falls nicht anders erläutert.

Schräge Einschüsse sind in MD-Simulationen für die Winkel $\alpha=20^{\circ}, \alpha=45^{\circ}$ und $\alpha=65^{\circ}$ bei Umgebungstemperaturen von $T=10 \mathrm{~K}$ und $T=300 \mathrm{~K}$ durchgeführt worden. Da sich die Streuung der Ergebnisse für $\delta$ als sehr groß erwiesen hat, sind pro 
Parameterkombination mehrere hundert Einschüsse durchgeführt worden. Dabei wird die Startposition sowie die Sorte des eingeschossenen Atoms zufällig gewählt, was in einem Programmcode von S. G. Mayr realisiert ist, der hier eingesetzt wird. Die Zeit zwischen zwei Einschüssen beträgt 30 ps, falls nicht anders erläutert. Diese Zeit ist ausreichend, denn die ballistischen Prozesse finden nur in einem wesentlich kürzeren Zeitrahmen statt, der in der Größenordnung 1 ps liegt (vgl. Kap. 7.1.1). Für jeden Einschuss wird der Wert von $\delta$ berechnet und zwar für Oberflächenschichten dreier verschiedener Dicken, nämlich für die gesamte Schicht, die obere Hälfte und die oberen zehn Prozent. Bei den zunächst verwendeten Schichten mit einer Dicke von ungefähr $80 \AA$ entspricht das Dicken von $80 \AA, 40 \AA$ und $8 \AA$. In die Summation gehen stets nur Atome ein, die vor und nach dem Einschuss zur Schicht gehören, so dass das eingeschossene Atom selbst und die gesputterten Atome nicht berücksichtigt werden. Des Weiteren sind Simulationen zum schrägen Einschuss auf zwei verschiedene Arten durchgeführt worden. Eine Möglichkeit ist, auf jeweils dieselbe Startkonfiguration ein Atom zu schießen und zwar mit verschiedenen Startpositionen für das eingeschossene Atom. Die zweite Möglichkeit besteht darin, auf eine Startkonfiguration im Abstand der Simulationszeit pro Einschuss sukzessive viele Atome mit unterschiedlichen Startpositionen zu schießen.

Es sind verschiedene Randbedingungen getestet worden. Beiden gemeinsam ist, dass in x- und y-Richtung periodische Randbedingungen herrschen, während in z-Richtung freie Oberflächen vorhanden sind. Die erste Art der Randbedingungen besteht darin, eine frei schwebende Schicht zu simulieren und die Temperaturkontrolle an den periodischen Rändern wirken zu lassen. Das entspricht den Bedingungen, die auch bei den senkrechten Einschüssen gelten. Beim schrägen Einschuss kann dann wegen Impulsübertragung die gesamte Schicht einen Impuls entlang der x-Richtung erlangen. Dann muss bei der Berechnung der lateralen Verschiebung allerdings immer die mittlere Bewegung der Schicht berücksichtigt werden, die man aus der mittleren Bewegung der Atome am unteren Ende der Probe erhält, weil diese Atome von dem eingeschossenen Atom und der resultierenden Stoßkaskade nicht direkt beeinflusst sind. Die zweite Art der Randbedingungen beim schrägen Beschuss ist, den unteren Rand der Schicht (etwa 2 bis 4 Atomlagen) festzuhalten und die Temperaturkontrolle nur an diesem unteren Rand einzusetzen. Dabei muss die Temperaturkontrolle auf einige Atomlagen oberhalb der festgehaltenen Atome angewendet werden. Es ist überprüft worden, dass die Wahl der Randbedingungen keinen signifikanten Einfluss auf die Ergebnisse hat. Im weiteren Verlauf der Arbeit wird beim schrägen Beschuss die zweite Möglichkeit eingesetzt, wenn es nicht explizit anders beschrieben wird.

Um ausschließen zu können, dass die verwendete Simulationszelle zu klein ist, muss gewährleistet sein, dass das Ergebnis nicht durch Atome beeinflusst wird, die entlang der x-Achse eine Entfernung zurücklegen, die größer als die halbe Länge der Simulationszelle ist. Deshalb wird für jeden Einschuss analysiert, ob es solche Atome gibt. Zusätzlich wurde der schräge Einschuss mit größeren Zellen simuliert. Diese großen Simulationszellen haben eine Ausdehnung von $196 \AA$ in x- und y-Richtung und $98 \AA$ in z-Richtung. 


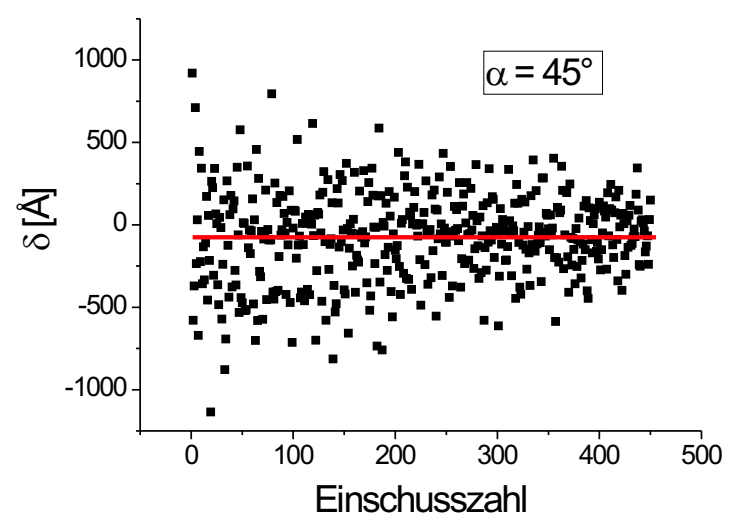

Abbildung 7.10: Summe der atomaren Verschiebungen $\delta$ im Bereich der oberen $8 \AA$ für sukzessiven Beschuss einer CuTi Oberfläche unter einem Winkel von $\alpha=45^{\circ}$ bei $T=300 \mathrm{~K}$.

\subsubsection{Ergebnisse zum Vergleich von viskosem Oberflächenfließen und ballistischem Transport}

Für die Oberflächenenergie ergibt sich mit Gleichung (7.9) für $T=10 \mathrm{~K}$ und $T=300 \mathrm{~K}$ ein übereinstimmender Wert von $\tilde{\gamma}=1.14 \mathrm{~J} / \mathrm{m}^{2}$.

Für die atomaren Verschiebungen $\delta$ sollen die Ergebnisse vorgestellt werden, die sich ergeben, wenn die Oberfläche sukzessive mit einer Vielzahl von Atomen beschossen wird. Für eine Begründung der Bevorzugung dieser Methode sei auf die Diskussion in Kap. 7.2.3 verwiesen. Dort wird auch auf die andere Methode eingegangen und erläutert, warum die Simulation der sukzessiven Bestrahlung die physikalisch sinnvollere Methode ist. Die einzelnen Werte für die Summe der durch einen Einschuss bedingten Verschiebungen in x-Richtung $\delta$ sind in Abb. 7.10 für jeden Einschuss aufgetragen. Dabei werden zwei Dinge deutlich: Zum einen ist die Streuung der Werte sehr groß, so dass eine Vielzahl von Einschüssen nötig ist, um verlässliche Resultate zu erhalten; zum anderen ist nicht erkennbar, dass sich der mittlere Wert von $\delta$ als Funktion der Anzahl von Einschüssen ändert. Für $T=300 \mathrm{~K}$ sind die Werte für $\delta$ mit den zugehörigen Fehlern der Mittelwerte, die sich nach 450 Einschüssen ergeben, für die verschiedenen Einschusswinkel und die verschiedenen analysierten Schichtdicken in Tab. 7.4 eingetragen. Die entsprechenden Ergebnisse bei einer Umgebungstemperatur von $T=10 \mathrm{~K}$ finden sich in Tab. 7.5. Dabei fällt auf, dass der Fehler umso größer wird, je dicker die Schicht ist, in der die Verschiebungen analysiert werden. Außerdem sind die Fehler bei $T=300 \mathrm{~K}$ stets größer als bei $T=10 \mathrm{~K}$. Bezogen auf die Einschusswinkel ist erkennbar, dass sich für $\alpha=20^{\circ}$ stets die betragsmäßig kleinsten Werte ergeben. Bei $\alpha=45^{\circ}$ sind die Werte betragsmäßig am größten. Beim Einfluss der analysierten Schichtdicke auf das Ergebnis ist festzustellen, dass sich dem Betrage nach die Werte zwischen der dünnsten und der mittleren Schichtdicke erhöhen und zwischen der mittleren und der größten Schichtdicke nahezu (bei Berücksichtigung der zugehörigen Fehler) konstant bleiben. Aus diesem Grund (siehe 


\begin{tabular}{|l|l|l|l|}
\hline & $\delta\left(20^{\circ}\right)[\AA]$ & $\delta\left(45^{\circ}\right)[\AA]$ & $\delta\left(65^{\circ}\right)[\AA]$ \\
\hline$d=8 \AA$ & $-46 \pm 16$ & $-71 \pm 13$ & $-50 \pm 9$ \\
\hline$d=40 \AA$ & $-80 \pm 55$ & $-99 \pm 45$ & $-81 \pm 40$ \\
\hline$d=80 \AA$ & $-79 \pm 68$ & $-96 \pm 61$ & $-81 \pm 53$ \\
\hline
\end{tabular}

Tabelle 7.4: Werte der Summen lateraler Verschiebungen $\delta$ für verschiedene Einschusswinkel und verschiedene Dicken $d$ der analysierten Bereiche als Durchschnittswerte nach jeweils 450 Einschüssen bei einer Umgebungstemperatur von $T=300 \mathrm{~K}$.

\begin{tabular}{|l|l|l|l|}
\hline & $\delta\left(20^{\circ}\right)[\AA]$ & $\delta\left(45^{\circ}\right)[\AA]$ & $\delta\left(65^{\circ}\right)[\AA]$ \\
\hline$d=8 \AA$ & $-46 \pm 10$ & $-76 \pm 9$ & $-72 \pm 6$ \\
\hline$d=40 \AA$ & $-69 \pm 27$ & $-107 \pm 24$ & $-80 \pm 16$ \\
\hline$d=80 \AA$ & $-69 \pm 32$ & $-107 \pm 28$ & $-78 \pm 20$ \\
\hline
\end{tabular}

Tabelle 7.5: Werte der Summen lateraler Verschiebungen $\delta$ für verschiedene Einschusswinkel und verschiedene Dicken $d$ der analysierten Bereiche als Durchschnittswerte nach jeweils 450 Einschüssen bei einer Umgebungstemperatur von $T=10 \mathrm{~K}$.

dazu auch die zugehörige Diskussion in Kap. 7.2.3) werden die Übergangswellenlängen gemäß Gleichung (7.8) nur für die kleinste und die mittlere Schichtdicke angegeben. Diese Resultate sind in Tab. 7.6 dargestellt. Als wesentliche Tendenz zeigt sich, dass sich die Übergangswellenlänge mit größerem Winkel zu größeren Werten verschiebt. Des Weiteren ergeben sich bei $T=10 \mathrm{~K}$ kleinere Werte für $\lambda_{c}$ als bei $T=300 \mathrm{~K}$. Während die Werte von $\delta$ im Rahmen der Genauigkeit nicht temperaturabhängig sind, zeigt die strahlungsinduzierte Viskosität, die in die Berechnung von $\lambda_{c}$ eingeht, größere Werte für kleinere Temperaturen (siehe Tab. 7.1).

Die Simulationen mit größeren Zellen ergeben im Rahmen des Fehlerbalkens gleiche Ergebnisse und es zeigt sich außerdem, dass sich nur selten Atome weiter bewegen als die halbe Kantenlänge der kleinen Zelle und somit die Ergebnisse nicht wesentlich beeinflussen. Daher sind die Simulationszellen mit ungefähr $80 \AA$ Kantenlänge auch für den schrägen Einschuss groß genug.

\subsubsection{Vergleich von glättenden und aufrauenden Mechanismen}

Methode Als einziger deterministischer aufrauender Mechanismus ist in Kap. 4.5.1 die Sputtererosion angesprochen worden. Da dieser Mechanismus in der mathematischen Formulierung dieselbe Strukturabhängigkeit wie das ballistische Glätten zeigt, ist das Verhältnis beider Effekt nicht von der Strukturgröße abhängig. Für das Verhältnis gilt unter Verwendung der Gleichungen (4.17) und (4.24):

$$
\Delta_{3}:=\left|\frac{\left(b_{2} q^{2}\right)_{\text {ballistischer Transport }}}{\left(b_{2} q^{2}\right)_{\text {Sputtererosion }}}\right|=\left|\frac{-F \Omega\left|\frac{\delta}{\tan (\alpha)}\right| q^{2}}{\frac{F a_{E} Y}{2 n} q^{2}}\right|=\left|\frac{\delta}{\tan (\alpha)}\right| \frac{2}{a_{E}} \frac{1}{Y} .
$$




\begin{tabular}{|l|l|l|l|}
\hline & $\lambda_{c}\left(20^{\circ}\right)[\mathrm{nm}]$ & $\lambda_{c}\left(45^{\circ}\right)[\mathrm{nm}]$ & $\lambda_{c}\left(65^{\circ}\right)[\mathrm{nm}]$ \\
\hline$d=8 \AA, T=10 \mathrm{~K}$ & $37 \pm 5$ & $48 \pm 5$ & $72 \pm 6$ \\
\hline$d=40 \AA, T=10 \mathrm{~K}$ & $31 \pm 7$ & $41 \pm 6$ & $69 \pm 9$ \\
\hline$d=8 \AA, T=300 \mathrm{~K}$ & $84 \pm 16$ & $111 \pm 13$ & $195 \pm 22$ \\
\hline$d=40 \AA, T=300 \mathrm{~K}$ & $63 \pm 22$ & $94 \pm 23$ & $152 \pm 39$ \\
\hline
\end{tabular}

Tabelle 7.6: Übergangswellenlängen $\lambda_{c}$ für verschiedene Einschusswinkel und verschiedene Dicken $d$ der analysierten Bereiche bei Umgebungstemperaturen von $T=10 \mathrm{~K}$ und $T=300 \mathrm{~K}$.

\begin{tabular}{|l|l|l|}
\hline & $Y$ & $\Delta_{3}$ \\
\hline$\alpha=20^{\circ}$ & 3.2 & $3.5 \pm 1.4$ \\
\hline$\alpha=45^{\circ}$ & 4.9 & $1.3 \pm 0.3$ \\
\hline$\alpha=65^{\circ}$ & 5.2 & $0.42 \pm 0.10$ \\
\hline
\end{tabular}

Tabelle 7.7: Sputterausbeute und Verhältnis von ballistischem Glätten zur Aufrauung durch Sputtererosion nach jeweils 450 Einschüssen bei einer Umgebungstemperatur von $T=10 \mathrm{~K}$.

Der Bereich $a_{E}$, in dem ein Energieeintrag auftritt, kann mit der Dicke des flüssigen Bereichs $a_{z}$ identifiziert werden. Die Sputterausbeute $Y$ wird für verschiedene Winkel bestimmt, indem bei der sukzessiven Bestrahlung (bei $T=10 \mathrm{~K}$ ) nach einer Vielzahl von Einschüssen abgezählt wird, wie viele Atome sich oberhalb der Probe befinden und somit als gesputtert gelten. Hier können die Ergebnisse der niedrigen Umgebungstemperatur verwendet werden, weil beide Effekte nicht temperaturabhängig sind und insbesondere bei der Berechnung des ballistischen Transports die Fehler bei der niedrigen Temperatur kleiner sind. Die Temperaturunabhängigkeit des ballistischen Effekts ist in Kap. 7.1.3.5 gezeigt. Dass sich die Sputterausbeute deutlich unterhalb der Schmelztemperatur nicht wesentlich als Funktion der Temperatur ändert, ist aus der Literatur für vergleichbare Systeme bekannt [AB81] und konnte hier auch durch die Simulationen bei beiden untersuchten Temperaturen bestätigt werden.

Ergebnisse Nach jeweils 450 Einschüssen ist der Fehler der durchschnittlichen Sputterausbeute so klein, dass er verglichen mit dem Fehler von $\delta$ nicht mehr wesentlich zum Gesamtfehler beiträgt. Es sind an dieser Stelle jeweils die Werte bei $T=10 \mathrm{~K}$ verwendet worden, wobei für $\delta$ die obere Probenhälfte ausgewertet worden ist. Die Resultate für die Sputterausbeute und das betragsmäßige Verhältnis von ballistischem Glätten zur Sputtererosion sind in Tab. 7.7 dargestellt. Demnach ist der Glättungseffekt allein durch den ballistischen Transport größer oder gleich dem Aufrauungseffekt durch Sputtererosion, solange Steigungswinkel von $20^{\circ}$ oder bis zu $45^{\circ}$ der Oberflächenmorphologie entsprechen. 


\subsection{Diskussion der Ergebnisse zur Bestrahlung amorpher CuTi Oberflächen}

\subsubsection{Allgemeine Phänomene}

Zunächst ist als Kriterium für die Zuordnung der Atome zur festen bzw. flüssigen Phase ein rein kinetisches Kriterium verwendet worden, dass nur auf den kinetischen Energien einzelner Atome und deren lokalen Umgebungen begründet ist. Die Interpretation der Entwicklung der Anzahl flüssiger Atome ist bereits in die Methode eingegangen, mit der die Anzahl flüssiger Atome im Spike $N_{l i q}$ sowie dessen Lebensdauer $\tau_{l i q}$ berechnet wird. Der Zeitpunkt des Übergangs vom Maximalwert in das Plateau in Abb. 7.1 stimmt ungefähr mit der Zeitskala, die in [AR98] für den Übergang vom ballistischen Regime zum „thermal spike“ gefunden wird, überein, denn in beiden Fällen liegt die Übergangszeit ungefähr bei 400 fs.

Der Zeitraum, in dem die Anzahl flüssiger Atome ungefähr konstant bleibt, liegt deutlich über dem für thermische Schwingungsdauern typischen. Thermische Schwingungen existieren mit Schwingungsdauern im Zeitbereich von etwa 100 fs [SGB80; SR83] (siehe auch Kap. 6.2.1). Bei der hauptsächlich untersuchten Einschussenergie $E=1 \mathrm{keV}$ liegt die Lebensdauer der aufgeschmolzenen Bereiche mit $\tau_{\text {liq }}(T=10 \mathrm{~K})=2216 \pm 186 \mathrm{fs}$ und $\tau_{l i q}(T=300 \mathrm{~K})=5839 \pm 239$ fs deutlich darüber. Für die durchgeführten Einschüsse bei verschiedenen Energien bei einer Umgebungstemperatur von $T=10 \mathrm{~K}$ zeigt die Lebensdauer der aufgeschmolzenen Bereiche bereits ab $E=50 \mathrm{eV}$ einen Wert oberhalb der Zeit von 100 fs, so dass im Rahmen des kinetischen Kriteriums von einem tatsächlich existierenden flüssigen Zustand gesprochen werden kann.

Dass die als flüssig identifizierten Atome einen zusammenhängenden, kompakten Bereich bilden, stimmt mit den Ergebnissen für die Bestrahlung kristalliner Metalle in MDSimulationen überein $\left[\mathrm{NGA}^{+} 98\right]$. Diese Eigenschaft führt neben der zuvor diskutierten Lebensdauer dazu, dass in metallischen Systemen im untersuchten Energiebereich von ausgedehnten (bezüglich der kinetischen Energie) flüssigen Bereichen gesprochen werden kann.

Die doppeltlogarithmische Auftragung der Lebensdauern als Funktion der Einschussenergie in Abb. 7.11 zeigt, dass die Lebensdauer mit der Energie im untersuchten Energiebereich gemäß $\tau_{l i q} \propto E^{\epsilon}$ skaliert mit $\epsilon=0.66 \pm 0.03$. Wie in Kap. 7.1.1 erwähnt wird $\epsilon=2 / 3$ für einen kugelförmigen und $\epsilon=1$ für einen zylinderförmigen "thermal spike“ erwartet [AR98]. Daher kann hier gefolgert werden, dass im untersuchten Energiebereich ein kugelförmiger „thermal spike“ entsteht. Dieser Fall wird eher im Bereich niedriger Einschussenergien erwartet, während bei hohen Energien die Probe entlang der Spur des eingeschossenen Atoms aufschmilzt, so dass sich ein zylinderförmiger flüssiger Bereich bildet. So konnte für reines $\mathrm{Cu}$ eine lineare Abhängigkeit im Bereich höherer Energien $(E>1 \mathrm{keV})$ gefunden werden [AR98]. Damit ist beim hier untersuchten CuTi die Einschussenergie $1 \mathrm{keV}$ noch eher dem Bereich der kugelförmigen Gestalt des „thermal spikes" zuzuordnen, wie es auch die Abbildungen 7.1 und 7.3 zeigen. 


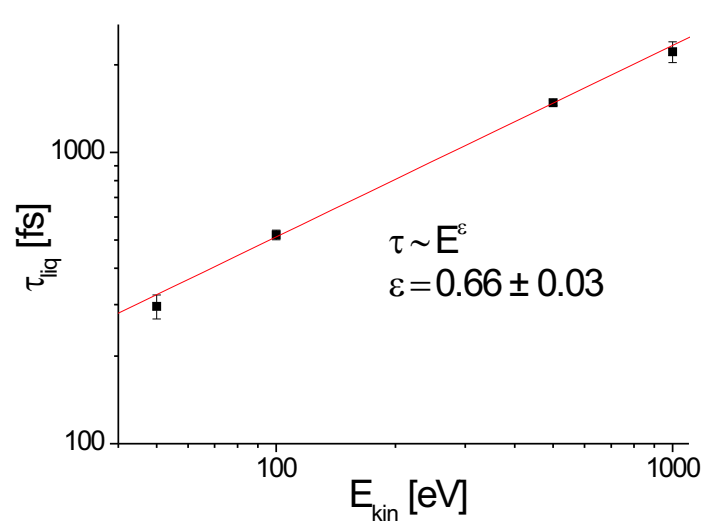

Abbildung 7.11: Doppeltlogarithmische Darstellung der Lebensdauern flüssiger Bereiche als Funktion der Einschussenergie für CuTi Schichten bei $T=10 \mathrm{~K}$ (aus Abb. 7.2).

\subsubsection{Analyse der atomaren Dynamik}

Die Verteilung der atomaren Verschiebungsweiten kann Informationen über die Art der Dynamik liefern. Welche Schlussfolgerungen aus der Verteilung gezogen werden können, ist bereits im Kapitel über thermisch angeregte Prozesse beschrieben worden (siehe Kap. 6.1.3). Wie dort erklärt ergibt sich eine Verteilung von der Form einer radial gemittelten Gaußverteilung, wie sie durch Gleichung (6.8) beschrieben wird, aus einer rein kooperativen Bewegung ohne bevorzugte Längenskala. Für die thermische Anregung bei $T=450 \mathrm{~K}$ konnte für $\mathrm{Cu}$ Atome im Bereich der Oberfläche ein zweites Maximum im Bereich des Abstands nächster Nachbarn gefunden werden, das sich als Indiz für die Existenz von Einzelsprüngen interpretieren lässt und sicher anzeigt, dass beim vorherrschenden Transportmechanismus der Abstand nächster Nachbarn eine bevorzugte Länge der Bewegung darstellt. Dass die Verteilung im Falle des Einschusses bei $T=10 \mathrm{~K}$ der Gaußverteilung deutlich näher kommt als die der thermisch angeregten Bewegung, zeigt, dass es sich bei der strahlungsinduzierten Dynamik um einen kooperativen Prozess handelt, bei dem keine ausgezeichnete Länge der Bewegung existiert, denn im Bereich des Nachbarabstandes ist keine Andeutung eines zusätzlichen Maximums erkennbar. Genau dieses Verhalten entspricht dem der Bewegung in einer Flüssigkeit [Wah91]. Die Art der Dynamik unterscheidet sich demnach für die verschiedenen Arten der Anregung. Mit diesem Ergebnis ist ein weiterer Hinweis dafür geliefert, dass durch die Bestrahlung nicht nur angeregte Atome existieren, die lediglich nach einem Energiekriterium als flüssig bezeichnet werden können, sondern sich auch in ihrer Dynamik wie eine Flüssigkeit verhalten.

Der Vergleich der strahlungsinduzierten und thermisch aktivierten Dynamik zeigt, dass die mittlere quadratische Verschiebung bei beiden Prozessen für die gewählten Parameter (Temperatur, Zeit, Einschussenergie) von gleicher Größe ist, weil sich die Hauptmaxima der angepassten Kurven an derselben Stelle befinden, und für deren Position 
gilt: $r_{\max }=\sqrt{4 D t}=\sqrt{\frac{1}{6}\left\langle\left(\Delta \overrightarrow{r_{i}}-\left\langle\Delta \overrightarrow{r_{i}}\right\rangle\right)^{2}\right\rangle}$ (siehe Kap. 6.1.3). Der Beitrag zum Transport ist also in beiden Fällen fast gleich, aber die Art der Dynamik ist unterschiedlich.

Die Untersuchung der Dynamik liefert somit das Ergebnis, dass während des „,thermal spikes" ein kooperativer Prozess wie Fließen vorherrscht. Somit sollten Fließprozesse im untersuchten Energiebereich von Bedeutung sein. Allerdings ist mit diesem Resultat noch nicht ausgeschlossen, dass im Anschluss an den „thermal spike“ beim Übergang des Systems zum Ausgangszustand noch weitere Prozesse beteiligt sind. Deshalb sind die Untersuchungen im Rahmen der Kontinuumsmodelle wichtig für quantitative Resultate. Dadurch, dass hier die Existenz flüssiger Bereiche gezeigt werden konnte, ist die Anwendung von Kontinuumsmodellen, die viskoses Fließen beschreiben, gerechtfertigt.

\subsubsection{Untersuchung der Prozesse im Rahmen von Kontinuumsmodellen}

Fließen und Oberflächendiffusion Zur Modellierung von Fließprozessen soll zunächst daran erinnert werden, dass es zwei Grenzfälle gibt, die durch das Verhältnis der Dicke des flüssigen Oberflächenfilms und der Größe der Oberflächenstrukturen gegeben sind (siehe Kap. 4.5.4). Da beide Fließprozesse auf einem Transport in zueinander senkrechten Richtungen basieren und die die Dynamik des viskosen Fließens beschreibende Navier-Stokes-Gleichung in der verwendeten Näherung linear ist, können beide Fließprozesse als sich linear überlagernd angesehen werden. Im betrachteten Energiebereich liegt die Dicke des flüssigen Bereichs gemäß Tab. $7.1 \mathrm{im}$ Bereich von $3 \mathrm{~nm}$ bis $4 \mathrm{~nm}$. Die Strukturgrößen, für die Glättung durch Ionenbestrahlung in Kap. 5 experimentell untersucht wird, liegen bei lateralen Korrelationslängen von ungefähr $20 \mathrm{~nm}$ und der vertikale Abstand von Tälern zu Hügeln liegt bei etwa 10 nm [RMS97; RML $\left.{ }^{+} 00\right]$ (siehe auch Kap. 5). Somit ist für die hier untersuchten Phänomene das Oberflächenfließen im Vergleich zum Volumenfließen der dominierende Prozess. Wenn also die Dominanz von Oberflächenfließen über Oberflächendiffusion oder ballistischen Transport gezeigt werden kann, so ist damit allgemein die Dominanz von Fließprozessen gezeigt.

Beim Vergleich von viskosem Oberflächenfließen und Oberflächendiffusion hat sich ergeben, dass für sämtliche Berechnungsmethoden der Diffusionskoeffizienten der Fließprozess dominiert. Dieses Ergebnis gilt unabhängig von der Strukturgröße, weil beide Prozesse in gleicher Weise von der Form des Höhenprofils abhängig sind.

Die drei verschiedenen Methoden zur Berechnung der strahlungsinduzierten Diffusionskoeffizienten haben jeweils ihre spezifischen Vorzüge und auch Nachteile. Beim Vergleich von Methode 1 und Methode 2 spricht für Methode 2, dass hier die Temperatur realistischer wiedergegeben wird. Allerdings muss man davon ausgehen, dass sich allein durch die Aufteilung in mehrere Zeitintervalle der effektive Diffusionskoeffizient erhöht, wenn er so wie hier geschehen berechnet wird. Der Grund dafür ist, dass alle atomaren Bewegungen - und somit auch thermische Schwingungen - berücksichtigt werden. Thermische Schwingungen liefern für jedes Intervall einen ähnlich großen Beitrag, so dass insgesamt der Beitrag durch thermische Schwingungen mit der Anzahl von Zeitintervallen wächst. Während der Simulation von Einschüssen und der Analyse der induzierten 
atomaren Dynamik ist es sehr schwierig, einen Diffusionskoeffizienten zu bestimmen, in den keine Beiträge thermischer Schwingungen eingehen. Für die Untersuchung thermisch angeregter Effekte gibt es die in Kap. 6.5 diskutierten Möglichkeiten zum Herausfiltern thermischer Schwingungen. So wurden für die Simulationen zur Bestrahlung zusätzlich auch Diffusionskoeffizienten unter Verwendung eines Abstands $r_{c}$ berechnet, unterhalb dessen Bewegungen als thermische Schwingungsbeiträge interpretiert werden und nicht zum Diffusionskoeffizienten beitragen, wenngleich diese Methode in amorphen Systemen problematisch ist (siehe Kap. 6.5 und [KS04]). Dafür wurde $r_{c}$ mit einem Abstand identifiziert, unterhalb dessen sich die wesentlichen thermischen Schwingungen bei thermischer Anregung abspielen. Da sich ohne Verwendung dieses Kriteriums eine obere Grenze für den Diffusionskoeffizienten und mit diesem Wert bereits die Dominanz des Oberflächenfließens gegenüber der Oberflächendiffusion ergibt, ist hier die Verwendung des Kriteriums nicht notwendig. Die Dominanz des Oberflächenfließens würde sich mit dem Kriterium lediglich weiter verstärken. Die anderen in Kap. 6.5 erwähnten Methoden zur Eliminierung thermischer Beiträge sind bei der Simulation der Bestrahlung von Oberflächen nicht anwendbar. So ist eine Mittelung der Atompositionen [KS04] über mehrere Schwingungsperioden nicht sinnvoll, weil die lokale Temperatur räumlich und zeitlich stark variiert und außerdem kurz nach dem Einschuss in kurzer Zeit relativ weite Bewegungen stattfinden können. Die Methode des raschen Abkühlens zur Bestimmung der atomaren Gleichgewichtspositionen [Tei92] ist unter den verwendeten Randbedingungen mit Oberflächen wegen der Druckregelung problematisch. Alles in allem ist für die erörterte Fragestellung die Berechnungsmethode, mit der die präsentierten Ergebnisse erzielt worden sind, genau genug, denn die berechneten Diffusionskoeffizienten können hinsichtlich der Beiträge thermischer Schwingungen als obere Grenzen angesehen werden und weitere Methoden zur Eliminierung thermischer Schwingungen müssen nicht untersucht werden, weil sich bereits mit diesen oberen Grenzen für die Diffusionskoeffizienten die Dominanz des Oberflächenfließens ergibt.

Für $T=300 \mathrm{~K}$ ist mit allen Berechnungsmethoden die Dominanz des Oberflächenfließens sehr deutlich, weil das Verhältnis der relativen Gewichte zwischen Oberflächenfließen und Oberflächendiffusion deutlich größer als eins ist. Bei $T=10 \mathrm{~K}$ ergibt sich zwar auch eine Dominanz des Oberflächenfließens, aber sie ist nicht so deutlich wie bei der höheren Temperatur. Begründen lässt sich diese Temperaturabhängigkeit mit der Form der Diffusion. Wie in Abb. $7.7 \mathrm{zu}$ erkennen ist, entwickelt sich die mittlere quadratische Verschiebung nicht linear als Funktion der Zeit. Stattdessen erfolgt zunächst ein sehr steiler Anstieg im Zeitbereich der anfänglichen ballistischen Effekte und des „thermal spikes" (vgl. auch Abb. 7.6). Danach ist nur noch ein sehr viel kleinerer Anstieg festzustellen, so dass sich die mittleren quadratischen Verschiebungen nach 50 ps und 1000 ps nur wenig unterscheiden. Der steile Anstieg zu Beginn lässt sich durch ballistische Effekte und Vorgänge im Bereich des „,thermal spike“ erklären und die sollten keine starke Abhängigkeit von der Umgebungstemperatur zeigen. Insgesamt zeigt die mittlere quadratische Verschiebung somit nur eine schwache Temperaturabhängigkeit und die Beweglichkeit $D_{s} /\left(k_{B} T\right)$ wächst mit sinkender hier eingesetzter Umgebungstemperatur. Deshalb ist es besonders bei niedrigen Umgebungstemperaturen wichtig, die tatsächliche Temperatur entsprechend Methode 2 realistischer wiederzugeben, als es mit Methode 1 
möglich ist. Hier ergibt sich mit Methode 2 dementsprechend ein deutlich größerer Wert für $\Delta_{1}$ als mit Methode 1. Die Ergebnisse aus beiden Methoden unterschieden sich umso stärker, je weiter die Umgebungstemperatur und die in Methode 2 eingesetzte Glastemperatur auseinander liegen. Insbesondere bei sehr tiefen Temperaturen ist Methode 2 sehr viel realistischer als Methode 1.

Die Methoden 1 und 2 haben bei der Berechnung der Diffusionskoeffizienten der einzelnen Atomsorten gezeigt, dass $\mathrm{Cu}$ Atome immer einen höheren Diffusionkoeffizienten als Ti Atome haben. Dieses Verhalten stimmt mit dem der Diffusion bei thermischer Aktivierung überein (siehe Kap. 6.1). Insofern sollten auch bei der strahlungsinduzierten Dynamik dieselben Begründungen zulässig sein, die darauf beruhen, dass die $\mathrm{Cu}$ Atome kleiner als die Ti Atome sind (siehe Diskussion 6.2.2).

Methode 3 ist nur bei der höheren Temperatur anwendbar, weil thermisch aktivierte Diffusion vorhandener Adatome auf Metalloberflächen bei $T=300 \mathrm{~K}$ stattfindet und bei $T=10 \mathrm{~K}$ nicht [MT94; $\left.\mathrm{BEH}^{+} 01\right]$. Deshalb ist es auch nur bei der höheren Temperatur notwendig, die thermisch aktivierte Adatomdiffusion zu berücksichtigen. Die Anzahl der über das geometrische Kriterium bestimmten Adatome liegt im Rahmen der Erwartungen. Mit einem sehr ähnlichen Kriterium, bei dem zusätzlich noch ein Mindestabstand eingeführt ist, um den ein Atom über der ursprünglichen Oberfläche liegen muss, um als Adatom bezeichnet zu werden, werden für den Beschuss von $\mathrm{Al}(111)$ Oberflächen in der MD-Simulation mit verschiedenen Edelgasen bei gleicher Einschussenergie etwas kleinere Werte als in dieser Arbeit gefunden $\left(N_{\text {Adatom }}=7.1 \pm 1.0\right.$ für $\mathrm{Ar}^{+}$und $N_{\text {Adatom }}=7.6 \pm 1.6$ für $\left.\mathrm{Xe}^{+}\right)\left[\mathrm{BEH}^{+} 01\right]$. Der etwas höhere Wert $N_{\text {Adatom }}=17 \pm 3$ in Kap. 7.1.3.3 lässt sich dadurch begründen, dass hier kein zusätzlicher Mindestabstand verwendet wird. Es gibt auch experimentelle Untersuchungen zur Abschätzung der Anzahl pro Einschuss produzierter Adatome. Dafür werden atomar glatte kristalline Oberflächen beschossen und mit dem STM untersucht, so dass die Dichte und Größe von Inseln, die sich aus Adatomen gebildet haben, vermessen werden können [MT94; $\mathrm{BEH}^{+}$01]. Mit dieser Methode wird eine obere Grenze für die Anzahl von Adatomen bestimmt, weil der endliche Spitzenradius die Inseln größer erscheinen lässt, als sie tatsächlich sind. Für die Bestrahlung von $\mathrm{Al}(111)$ wird als Abschätzung aus STM Untersuchungen $12<N_{\text {Adatom }}<50$ angegeben $\left[\mathrm{BEH}^{+} 01\right]$. Für amorphe Oberflächen ist diese experimentelle Bestimmungsmethode nicht anwendbar, da eine amorphe Oberfläche stets eine atomare Rauigkeit aufweist und Inseln von Adatomen nicht so deutlich identifizierbar sind wie bei Kristallen. Insgesamt liegt die aus der Bestrahlung von amorphen CuTi Schichten über das geometrische Kriterium bestimmte Ausbeute an Adatomen im Rahmen dessen, was in der Literatur wenngleich für andere Materialien und andere Struktur - bekannt ist. Somit kann das verwendete Kriterium als eine realistische Methode angesehen werden.

Bei der aus dem verwendeten Energiekriterium bestimmten Anzahl von Adatomen, die pro Ion produziert werden, fällt auf, dass danach die Anzahl hergestellter Adatome ähnlich groß wie die der vernichteten ist. Dieses Verhalten entspricht nicht den Ergebnissen für kristalline Oberflächen und auch nicht den Erwartungen für amorphe Oberflächen. Wenngleich gerade auf amorphen Oberflächen durchaus vorstellbar ist, dass einer Relaxation der Oberfläche entsprechend lokal schwach gebundene Atome nach dem Einschuss fester gebunden sind, sollte sich insgesamt durch den Einschuss die Anzahl 
angeregter Atome erhöhen. Außerdem ist das Ergebnis für die Anzahl produzierter Adatome abhängig von der gewählten Energiegrenze, durch die Adatome bestimmt werden. Ist die Energiegrenze zu klein gewählt, würden jeweils Atome gezählt, die zum Teil weder zu Beginn noch am Ende tatsächlich Adatome sind. Insofern hängen die Ergebnisse kritisch vom Wert dieser Energiegrenze ab. Insgesamt erscheint das geometrische Kriterium zur Bestimmung von $N_{\text {Adatom }}$ realistischer. Es würde versagen, wenn die Oberfläche durch die Einschüsse merklich rau würde, aber das ist hier nicht der Fall.

Unter Berücksichtigung aller durchgeführten Methoden hat sich gezeigt, dass der Glättungsbeitrag von Oberflächenfließen deutlich größer als der der Oberflächendiffusion ist. Bereits zuvor ist erläutert worden, dass unter den gegebenen Bedingungen Oberflächenfließen einen dominierenden oder zumindest einen wesentlichen Anteil der möglichen Fließprozesse darstellt. Zusätzlich lässt sich auch der Glättungseffekt von Volumenfließen mit dem der Oberflächendiffusion vergleichen. Da beide Effekte durch unterschiedliche Abhängigkeiten vom Wellenvektor $q$ gekennzeichnet sind, wird es von der Wellenlänge der betrachteten Struktur abhängen, welcher Effekt dominiert. Als Quotient beider Effekte ergibt sich unter Berücksichtigung von Gl. (4.22) und Gl. (4.27) und mit Verwendung der in $\Delta_{1}$ zusammengefassten Konstanten in Gl. (7.2):

$$
\Delta_{4}:=\frac{\left(b_{1} q^{1}\right)_{\text {Volumenfließen }}}{\left(b_{4} q^{4}\right)_{\text {Oberflächendiffusion }}}=\frac{\frac{\gamma}{2 \eta} q}{\frac{D_{S} \gamma \Omega^{2} \nu}{k_{B} T} q^{4}}=\frac{3}{2} \frac{1}{a_{z}^{3} q^{3}} \Delta_{1} .
$$

Als Übergangswellenlänge $\lambda_{c}^{V F D}$, für die beide Prozesse (Volumenfließen VF und Oberflächendiffusion D) gleichen Beitrag zeigen $\left(\Delta_{4}=1\right)$, folgt daraus

$$
\lambda_{c}^{V F D}=\frac{2 \pi}{q_{c}}=2 \pi\left(\frac{2}{3} \frac{a_{z}^{3}}{\Delta_{1}}\right)^{1 / 3} .
$$

Damit ergeben sich für $T=300 \mathrm{~K}$ mit den Berechnungsmethoden 1, 2 und 3 zur Abschätzung des Beitrags der Oberflächendiffusion als Übergangswellenlängen $\lambda_{c}^{V F D}=3.9 \pm 0.4 \mathrm{~nm}, \lambda_{c}^{V F D}=3.8 \pm 0.3 \mathrm{~nm}$ bzw. $\lambda_{c}^{V F D}=5.1 \pm 0.4 \mathrm{~nm}$. Für Wellenlängen $\lambda>\lambda_{c}^{V F D}$, also für die meisten relevanten Strukturen, dominiert der Fließprozess. Somit ist gezeigt, dass selbst unter Bedingungen, bei denen Volumenfließen einen größeren Beitrag als Oberflächenfließen hat, für die meisten Oberflächenstrukturen der Fließprozess dominieren würde.

Für hohe Bestrahlungsenergien im MeV-Bereich dominiert bei den experimentell relevanten Strukturgrößen das Volumenfließen das Oberflächenfließen und von Bestrahlungen des metallischen Glases $\mathrm{Zr}_{65} \mathrm{Al}_{7.5} \mathrm{Cu}_{27.5}$ im MeV-Bereich ist die Dominanz des Volumenfließens als Glättungsprozess bekannt [MA01]. Dieses Resultat ist in Übereinstimmung mit dem Simulationsergebnis, wenn man annimmt, dass sich die Größen in Gleichung (7.11) durch Erhöhung der Einschussenergie nicht wesentlich ändert. Für das Produkt $D_{S} \eta$ ist diese Annahme wiederum durch die Ergebnisse aus [MAAA03] bestätigt und für die anderen Größen gilt sie ebenfalls.

Fließen und ballistisches Glätten Die berechnete Oberflächenenergie ist etwas kleiner als die aus der Literatur bekannten Werte für die einzelnen Elemente. So werden 
in [TM77] für $T=0 \mathrm{~K}$ als Werte $1.78 \mathrm{~J} / \mathrm{m}^{2}$ für $\mathrm{Cu}$ und $1.98 \mathrm{~J} / \mathrm{m}^{2}$ für Ti bzw. für die Schmelztemperatur $1.57 \mathrm{~J} / \mathrm{m}^{2}$ für $\mathrm{Cu}$ und $1.75 \mathrm{~J} / \mathrm{m}^{2}$ für Ti angegeben. In der zitierten Arbeit wird weiter begründet, dass die Entropie zwischen $T=0 \mathrm{~K}$ und der Schmelztemperatur $T_{m}$ von einer Größenordnung ist, die nur einen geringen Unterschied zwischen der Oberflächenenergie und der freien Oberflächenenergie verursacht $(<15 \%)$. Deshalb liefert die aus der Simulation berechnete Oberflächenenergie eine gute Näherung der freien Oberflächenenergie. Eigentlich ist in Gleichung (7.8) die freie Oberflächenenergie bei einer höheren Temperatur als der Umgebungstemperatur einzusetzen, weil der Fließprozess als Folge des Einschusses bei einer lokal erhöhten Temperatur in einer flüssigen Phase stattfindet. Wie zuvor erwähnt weicht der entsprechende Wert aber nicht um mehr als $15 \%$ vom eingesetzten ab [TM77].

Bei der Auswertung der atomaren Verschiebungen ist auffällig, dass deren Summe $\delta$ einen sehr kleinen Wert hat, wenn man ihn mit atomaren Abständen vergleicht und die Zahl der Atome, die bei der Summation berücksichtigt werden, bedenkt. Die Berechnung von $\delta$ für Zellen derselben Größe haben gezeigt, dass sich Werte in ähnlicher Größenordnung ergeben können, wenn kein Atom eingeschossen wird. Ohne Einschuss sollte im Mittel $\delta=0$ gelten, aber für eine bestimmte Konfiguration der Zelle und ein festes Zeitintervall der Untersuchung gibt es immer Abweichungen. Wenn diese Abweichungen von ähnlicher Größenordnung wie der durch Einschuss induzierte Effekt sind, würde der Effekt des Einschusses stark verfälscht, wenn für jeden Einschuss dieselbe Konfiguration der Zelle und immer derselbe Untersuchungszeitraum gewählt würde. Die Methode der sukzessiven Bestrahlung einer Probe hat gerade den Vorteil, dass vor jedem einzelnen Einschuss die Zellenkonfiguration eine andere ist. Deshalb sollten sich die Effekte in der Summe von atomaren Verschiebungen, die nicht durch den Einschuss bedingt sind, nach einer Vielzahl von Einschüssen mit verschiedenen Zellenkonfigurationen kompensieren. Damit ergibt sich dann im Durchschnitt vieler sukzessiver Einschüsse ein Wert, der allein durch die Einschüsse begründet ist.

Dass die Fehler von $\delta$ bei der höheren Temperatur größer werden, entspricht der Erwartung. Auch die Abweichungen von $\delta=0$ für eine Zelle ohne Einschuss werden größer. Außerdem werden die Abweichungen bei einer Simulationszelle ohne Einschuss größer, wenn die Zelle größer ist. Im Wesentlichen sollten für diese Abweichungen thermische Schwingungen verantwortlich sein. Dies macht sich bemerkbar, wenn viele Teilchen berücksichtigt werden, auf die die gerichteten ballistischen Effekte keinen Einfluss haben. Das gilt bei den durchgeführten Einschüssen für die untere Hälfte der Probe. Die Ergebnisse für $\delta$ in Abhängigkeit von der Dicke des analysierten Bereichs bestätigen dies, denn die Ergebnisse sind gleich, wenn die gesamte Probe oder nur die obere Hälfte untersucht wird. Allerdings wird der Fehler größer, wenn die gesamte Probe berücksichtigt wird. Insofern ist es für die Auswertung der Größe $\delta$ sinnvoll, einen Bereich der Probe zu analysieren, in dem zum einen alle ballistisch bedingten Bewegungen stattfinden und der zum anderen möglichst klein ist.

Dass sich die Ergebnisse für $\delta$ als Funktion der Umgebungstemperatur nicht signifikant unterscheiden, entspricht der Erwartung, denn ein ballistischer Effekt beruht auf gerichteten Stößen durch Impulsübertrag, während thermische Bewegung nicht an die Einschussrichtung gekoppelt ist. Der ballistische Prozess mit gerichteten Stößen sollte 
sich solange fortsetzen, bis die Energie der gestoßenen Teilchen soweit gesunken ist, dass sie im Bereich der Bindungsenergie der Atome liegt. Dieses Kriterium ist unabhängig von der Temperatur, so dass ballistische Effekte temperaturunabhängig sind. Anders als in den Untersuchungen in $\left[\mathrm{MGC}^{+} 05\right]$ zu kovalent gebundenen Systemen steigt der Wert von $\delta \cdot \cos (\alpha)$ (entspricht $\delta$ in der zitierten Arbeit) hier nicht wie dort gesehen an. Allerdings wird dort auch ein anderer Bereich von Steigungswinkeln (nur $\alpha \leq 20^{\circ}$ ) untersucht als hier. Für metallische Systeme sind keine quantitativen Untersuchungen dieses Effekts bekannt, so dass für eine weitere Diskussion dieses Aspekts auf Kap. 7.4.2 verwiesen wird.

Dass die Übergangswellenlängen für die höhere Temperatur größer sind, ist dadurch bedingt, dass die Viskosität bei der höheren Temperatur kleiner ist. Das Gewicht des viskosen Oberflächenfließens ist demnach größer. Wegen der Temperaturunabhängigkeit des ballistischen Effekts bleibt dessen Gewicht hingegen konstant. Insgesamt erhöht sich damit das relative Gewicht des Oberflächenfließens gegenüber dem ballistischen Glätten und die Übergangswellenlänge $\lambda_{c}$ wird größer, so dass Oberflächenfließen für einen größeren Bereich von Strukturgrößen mit $\lambda<\lambda_{c}$ dominiert.

Auch wenn bei der hier verwendeten Bestrahlungsenergie und den experimentell relevanten Strukturgrößen Oberflächenfließen im Vergleich zum Volumenfließen der dominierende Prozess ist, sollte kurz der Vergleich des Glättungseffekts vom Volumenfließen mit des ballistischen Transports vorgestellt werden. Die folgenden Abschätzungen gelten für den Fall, dass Volumenfließen dominant wäre. Als Quotient des Beitrags vom Volumenfließen zum ballistischen Glätten ergibt sich

$$
\Delta_{5}:=\frac{\left(b_{1} q^{1}\right)_{\text {Volumenfließen }}}{\left(b_{2} q^{2}\right)_{\text {ballistischer Transport }}}=\frac{\frac{\gamma}{2 \eta} q}{F \Omega\left|\frac{\delta}{\tan (\alpha)}\right| q^{2}}=\frac{\gamma}{2 \eta F \Omega}\left|\frac{\tan (\alpha)}{\delta}\right| \frac{1}{q} .
$$

Als Übergangswellenlänge $\lambda_{c}^{V F B}$, für die beide Prozesse (Volumenfließen VF und ballistischer Transport B) gleichen Beitrag zeigen $\left(\Delta_{5}=1\right)$, folgt daraus

$$
\lambda_{c}^{V F B}=\frac{2 \pi}{q_{c}}=2 \pi \frac{2 \eta F \Omega}{\gamma}\left|\frac{\delta}{\tan (\alpha)}\right| .
$$

Hier ist es nun so, dass für Strukturen mit einer Größe oberhalb der Übergangswellenlänge das Volumenfließen und unterhalb der ballistische Transport dominiert. Diese Übergangswellenlänge lässt sich durch die Übergangswellenlänge, die sich beim Vergleich von Oberflächenfließen (OF) und ballistischem Glätten (B) ergeben hat, ausdrücken. In der folgenden Gleichung ist $\lambda_{c}^{O F B}$ die in Gleichung (7.8) bestimmte Wellenlänge. Es gilt:

$$
\lambda_{c}^{V F B}=\frac{2 a_{z}^{3}(2 \pi)^{3}}{3 \lambda_{c}^{O F B^{2}}} .
$$

Damit ergeben sich bei $\mathrm{T}=300 \mathrm{~K}$ unter Berücksichtigung der ballistischen Effekte in der oberen Probenhälfte, auf die sich die ballistischen Prozesse auch tatsächlich beschränken, mit den Werten aus Tab. 7.6 für die verschiedenen Einschusswinkel Werte von 
$\lambda_{c}^{V F B}\left(20^{\circ}\right)=3.1 \pm 2.2 \mathrm{~nm}, \lambda_{c}^{V F B}\left(45^{\circ}\right)=1.4 \pm 0.7 \mathrm{~nm}$ und $\lambda_{c}^{V F B}\left(65^{\circ}\right)=0.53 \pm 0.28 \mathrm{~nm}$. Diese Übergangswellenlängen sind sehr klein, so dass fast alle Strukturen durch das Volumenfließen geglättet würden. Somit hat ballistisches Glätten keine bedeutende Rolle, wenn Volumenfließen relevant ist. Dieses Ergebnis stimmt mit der Erwartung überein, dass gerichtete ballistische Stöße weniger Einfluss haben, wenn der aufgeschmolzene Bereich (im Verhältnis zur Strukturgröße) größer ist. Auch dieses Ergebnis stimmt mit der für die Bestrahlung des metallischen Glases $\mathrm{Zr}_{65} \mathrm{Al}_{7.5} \mathrm{Cu}_{27.5}$ im $\mathrm{MeV}$-Energiebereich bekannten Dominanz des Volumenfließens [MA01] überein.

Zusammenfassung der Glättungsmechanismen Die wesentlichen Resultate aus der Kombination von Kontinuumsmodellen und MD-Simulationen lassen sich somit kurz zusammenfassen. Im hier betrachteten Energiebereich, in dem nur eine dünne Oberflächenschicht flüssig wird und somit Fließprozesse in Form von Oberflächenfließen stattfinden, dominiert das viskose Oberflächenfließen den Prozess der Oberflächendiffusion. Dies gilt unabhängig von der Strukturgröße zumindest in dem Bereich, in dem Oberflächenfließen der relevante Fließprozess ist. Insofern spielt Oberflächendiffusion als Glättungsprozess nur eine untergeordnete Rolle. Der Vergleich des viskosen Oberflächenfließens mit dem ballistischen Glätten hat gezeigt, dass es eine Übergangswellenlänge gibt, unterhalb der viskoses Oberflächenfließen und oberhalb der ballistisches Glätten die vorherrschende Rolle spielt. Für die experimentell relevanten Strukturgrößen dominiert eher der Fließprozess. Würde Volumenfließen als der dominierende Fließprozess angenommen, so würde er für die experimentell relevanten Strukturgrößen sowohl gegenüber Oberflächendiffusion als auch gegenüber ballistischem Glätten dominieren. Bei den hier betrachteten Energien ist zwar Oberflächenfließen als der Fließprozess anzunehmen, aber mit steigender Einschussenergie gibt es einen kontinuierlichen Übergang zum Volumenfließen [Orc62].

Vergleich glättender und aufrauender Mechanismen Beim Vergleich des ballistischen Glättens mit der Aufrauung durch Sputtererosion - also beider Terme die mit unterschiedlichem Vorzeichen proportional zu $\nabla^{2} h$ sind - ist deutlich geworden, dass der Glättungseffekt dominiert, wenn die Steigungswinkel kleiner als $45^{\circ}$ sind. Das ist in der Regel in der experimentellen Situation erfüllt (vgl. Kap. 5). Je nach Struktur dominieren die sehr kleinen oder die mittleren Steigungswinkel. Die Sputterausbeuten steigen beim schrägen Beschuss mit dem Winkel zwischen $20^{\circ}$ und $65^{\circ}$ relativ zur Substratnormalen an. Dies entspricht sowohl allgemeinen theoretischen Erwartungen nach Sigmund [Sig69] als auch experimentellen Resultaten [AB81]. Dementsprechend ist zu erwarten, dass bei senkrechtem Beschuss die Sputterausbeute noch niedriger als bei $20^{\circ}$ ist. Die Werte der Sputterausbeuten liegen ebenfalls im erwarteten Bereich zwischen 1 und 10 [AB81]. Da im experimentell relevanten Bereich von Steigungswinkeln das ballistische Glätten allein bereits das Aufrauen durch Sputtererosion überwiegt und zusätzlich die weiteren diskutierten Glättungsterme hinzukommen, ist insgesamt bei der senkrechten Bestrahlung von strukturierten Oberflächen metallischer Gläser ein Glättungseffekt zu erwarten. 


\subsection{Bestrahlung amorpher Si Oberflächen}

Bei der Untersuchung des atomaren Beschusses von Si Oberflächen werden dieselben Methoden wie zuvor bei CuTi verwendet, so dass an dieser Stelle direkt mit der Darstellung der Ergebnisse begonnen werden kann. Bestrahlt werden Si Oberflächen, die wie in Kap. 3.4 beschrieben hergestellt worden sind. Die Auslagerungszeit im Bulk-Zustand liegt

etwa 4 ns bei einer Temperatur von $T=1400 \mathrm{~K}$. Danach wird die Probe auf die gewünschte Temperatur gebracht, bei der die Bestrahlung simuliert werden soll. Das sind wie schon beim CuTi $T=10 \mathrm{~K}$ und $T=300 \mathrm{~K}$. Nach Herstellung der Oberflächen werden die Proben solange relaxiert, bis die Energie des Systems als Funktion der Zeit nicht signifikant weiter sinkt. Beschossen wird die Oberfläche stets mit Si Atomen.

\subsubsection{Allgemeine Phänomene}

Für die Bestrahlung von Si Oberflächen wird dieselbe Flüssigkeitsanalyse wie zuvor für $\mathrm{CuTi}$ verwendet. Dabei hängt das Ergebnis entscheidend davon ab, welche Energie als Grenzwert verwendet wird. Auch beim Si soll die Energie verwendet werden, die der Glastemperatur $T_{g}$ über $E=\frac{3}{2} k_{B} T_{g}$ entspricht. Entsprechend der Ergebnisse und Diskussionen zum Glasübergang von $\mathrm{Si}$ in Kap. 6.3 wird als Näherung $T_{g}=2600 \mathrm{~K}$ verwendet. In diesem Abschnitt ist die Bezeichnung von Atomen oder Bereichen als flüssig nur im Sinne der verwendeten kinetischen Definition zu verstehen. Für einen repräsentativen Einschuss bei einer Umgebungstemperatur von $T=10 \mathrm{~K}$ ist in Abb. 7.12 die zeitliche Entwicklung der Anzahl flüssiger Atome sowie die räumliche Anordnung der flüssigen Atome zu einer Zeit im Bereich des Plateaus $(t=246 \mathrm{fs})$ gezeigt. Nach einer Mittelung über mehrere Einschüsse ergeben sich für die Lebensdauern und die Anzahl flüssiger Atome die Werte $\tau_{l i q}=276 \pm 22$ fs bzw. $N_{l i q}=112 \pm 10$. Die Flüssigkeitsanalyse ergibt somit sowohl für die Lebensdauer flüssiger Bereiche als auch für die Anzahl der daran beteiligten Atome deutlich kleinere Werte als es bei derselben Einschussenergie beim metallischen Glas CuTi der Fall ist (siehe Kap. 7.1.1). Außerdem fällt in Abb. 7.12 auf, dass die als flüssig identifizierten Atome nicht einen zusammenhängenden, kompakten Bereich bilden, sondern auf voneinander getrennte Gebiete verteilt sind.

Wenngleich die Struktur der flüssigen Bereiche nicht kompakt ist, lässt sich auch hier eine Dicke $a_{z}$ berechnen, bis zu der flüssiges Verhalten auftritt. Dabei ergibt sich bei $T=10 \mathrm{~K}$ Umgebungstemperatur der Wert $a_{z}=38 \pm 3 \AA$.

Bei einer Umgebungstemperatur von $T=300 \mathrm{~K}$ ergeben sich etwas größere Werte für die Lebensdauer der flüssigen Bereiche und die Anzahl der daran beteiligten Atome: $\tau_{l i q}=363 \pm 39 \mathrm{fs}, N_{l i q}=162 \pm 16$. Für die Dicke der flüssigen Oberflächenschicht lautet das Ergebnis $a_{z}=33 \pm 4 \AA$. In qualitativer Übereinstimmung mit dem Verhalten bei $T=10 \mathrm{~K}$ bilden die als flüssig identifizierten Atome keinen zusammenhängenden Bereich. 
a.)

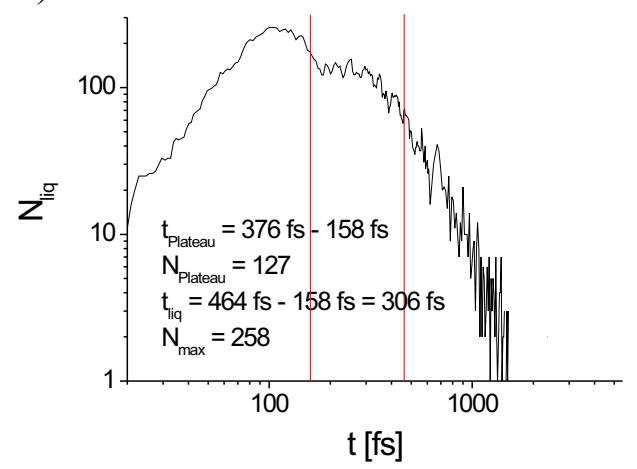

b.)

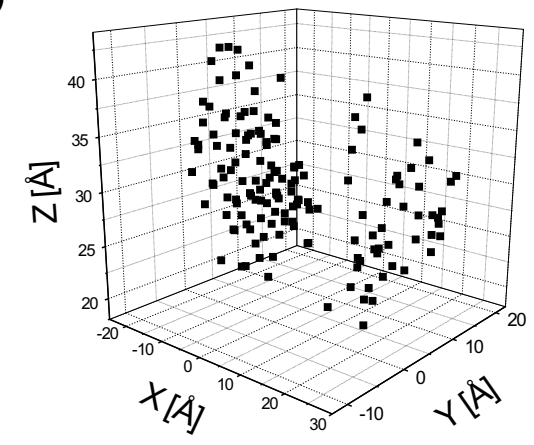

Abbildung 7.12: a.) Zeitliche Entwicklung der Anzahl flüssiger Atome (Die roten Linien markieren zeitlich den Anfangs- und Endpunkt des flüssigen Bereichs.) und b.) räumliche Anordnung der flüssigen Atome zum Zeitpunkt $t=246$ fs jeweils für denselben Einschuss der Energie $E=1 \mathrm{keV}$ bei einer Umgebungstemperatur von $T=10 \mathrm{~K}$.

\subsubsection{Analyse der atomaren Dynamik}

Eine Analyse der atomaren Dynamik anhand der Verteilung atomarer Verschiebungsweiten der (nach dem kinetischen Kriterium) flüssigen Atome im Oberflächenbereich kann beim Si nicht so aussagekräftig wie beim CuTi sein. Der Grund dafür ist, dass beim Si im Gegensatz zum CuTi bei thermischer Anregung kein eindeutiger Beweis der Existenz einer bevorzugten Verschiebungsweite gefunden werden konnte (vgl. Kap. 6). Insofern ist beim Si die Analyse der Verschiebungsweiten keine geeignete Methode, um das Verhalten einer Flüssigkeit im Unterschied zu thermisch aktivierter Diffusion nachzuweisen. Dass es tatsächlich ein durch den Beschuss induziertes flüssiges Verhalten im Si gibt, wird im späteren Kapitel 7.4 diskutiert und außerdem in Kap. 7.3.3.1 durch den Wert der strahlungsinduzierten Viskosität klar.

\subsubsection{Identifikation der Parameter aus Kontinuumsmodellen}

Analog zum entsprechenden Kapitel für das CuTi System werden im Folgenden die relativen Beiträge der glättenden Prozesse im Rahmen von Kontinuumsmodellen und Ergebnissen der MD-Simulationen quantitativ bestimmt.

\subsubsection{Viskoses Oberflächenfließen und Oberflächendiffusion}

Methoden Das Verhältnis des Glättungseffektes von viskosem Oberflächenfließen zu Oberflächendiffusion spiegelt sich im Wert des Parameters $\Delta_{1}$, der in Gleichung (7.2) definiert ist, wider. Die Werte der Diffusionskonstanten werden hier zunächst nur nach den in Kap. 7.1.3.2 als Methode 1 und Methode 2 erläuterten Verfahren berechnet. Der 


\begin{tabular}{|l|l|l|l|l|}
\hline & $\eta[\mathrm{Pa} \cdot \mathrm{dpa}]$ & $a_{z}[\AA]$ & $\Omega\left[\AA^{3}\right]$ & $\nu\left[\AA^{-2}\right]$ \\
\hline$T=300 \mathrm{~K}$ & $(9.66 \pm 0.02) \cdot 10^{8}$ & $33 \pm 4$ & 20.58 & 0.133 \\
\hline$T=10 \mathrm{~K}$ & $(12.35 \pm 0.03) \cdot 10^{8}$ & $38 \pm 3$ & 20.47 & 0.134 \\
\hline
\end{tabular}

Tabelle 7.8: Viskosität, vertikale Ausdehnung des flüssigen Bereichs, mittleres atomares Volumen und atomare Oberflächendichte während der Bestrahlung von Si Proben.

Grund dafür, dass das Verfahren nach Methode 3 für Si erst in der späteren Diskussion erläutert wird, ist, dass keine genauen Daten für eine mittlere Diffusionslänge von Adatomen auf amorphen Si Oberflächen bekannt sind.

Ergebnisse Die Werte für das atomare Volumen $\Omega$ und die Oberflächendichte von Atomen $\nu$ ergeben sich direkt aus den Atompositionen in der Simulation. Die mittlere Dicke der Oberflächenschicht, in der flüssige Bereiche auftreten, ist bereits in Kap. 7.3.1 vorgestellt worden. Die Berechnungen der strahlungsinduzierten Viskositäten $\eta$ wurden von S. G. Mayr nach demselben Prinzip, wie es für CuTi in [MAAA03] beschrieben ist, durchgeführt. Die Ergebnisse dieser Größen sind für die beiden untersuchten Umgebungstemperaturen in Tab. 7.8 dargestellt.

Für die Umrechnungen zwischen den Einheiten s und dpa sind Ergebnisse aus SRIM Simulationen herangezogen worden, bei denen Si Schichten mit Si Atomen der Energie $1 \mathrm{keV}$ beschossen worden sind. Für $T=10 \mathrm{~K}$ ergibt sich der Umrechnungsfaktor $1.6025 \cdot 10^{-3} \frac{\mathrm{dpa}}{t_{\text {ges }}}$ und für $T=300 \mathrm{~K}$ entsprechend $1.606 \cdot 10^{-3} \frac{\mathrm{dpa}}{t_{\text {ges }}}$ mit der Simulationszeit pro Einschuss $t_{\text {ges }}$. Berücksichtigt wird dabei der Oberflächenbereich von ca. $5 \AA$, weil in demselben Bereich auch die Daten für die Berechnung der Diffusionskoeffizienten berücksichtigt werden.

Die Ergebnisse für die Quotienten aus Diffusionskoeffizienten und Temperatur zusammen mit den resultierenden Werten des Parameters $\Delta_{1}$ sind für die Berechnungsmethoden 1 und 2 in den Tabellen 7.9 bzw. 7.10 aufgelistet. In allen untersuchten Fällen gilt $\Delta_{1}>1$ und das bedeutet, dass das viskose Oberflächenfließen die Oberflächendiffusion dominiert. Das Ergebnis ist in allen Fällen sehr deutlich außer bei $T=10 \mathrm{~K}$ unter Verwendung der Berechnungsmethode 1. In den Ergebnissen für diese Berechnungsmethode fällt auf, dass die Werte der Diffusionskoeffizienten $D_{s}$, die in der Einheit $\frac{\mathrm{m}^{2}}{\mathrm{dpa}}$ der Varianz proportional sind, innerhalb des Fehlerbalkens für beide Umgebungstemperaturen gleich sind. Bei $T=300 \mathrm{~K}$ sind für die Diffusionskoeffizienten gemäß Berechnungsmethode 1 Simulationszeiten von 50 ps und 1000 ps ausgewertet worden. Dabei ergibt sich für die deutlich längere Zeit ein kaum größerer Diffusionskoeffizient. Beide Werte liegen noch innerhalb der Fehlerbalkens des jeweils anderen Wertes. Für Berechnungsmethode 2 wird daher nur die maximale Simulationszeit ausgewertet. Die Ergebnisse zeigen beim Vergleich der beiden Berechnungsmethoden, dass sich bei der tiefen Temperatur mit Methode 2, in der die Temperatur wesentlich realistischer wiedergegeben wird, ein deutlich größerer Wert für $\Delta_{1}$ ergibt als mit Methode 1. Bei Raumtemperatur führt die Aufteilung in mehrere Zeitintervalle dagegen zu einer Vergrößerung von $D_{s} / T$ und somit 


\begin{tabular}{|l|l|l|}
\hline & $D_{s} / T\left[10^{-20} \frac{\mathrm{m}^{2} /}{\mathrm{dpa} \cdot \mathrm{K}}\right]$ & $\boldsymbol{\Delta}_{\mathbf{1}}$ \\
\hline$T=300 \mathrm{~K}, \Delta t=1 \mathrm{~ns}$ & $(14.6 \pm 2.3) / 300$ & $\mathbf{6 3} \pm \mathbf{2 0}$ \\
\hline$T=300 \mathrm{~K}, \Delta t=50 \mathrm{ps}$ & $(13.1 \pm 2.3) / 300$ & $\mathbf{7 0} \pm \mathbf{2 2}$ \\
\hline$T=10 \mathrm{~K}, \Delta t=50 \mathrm{ps}$ & $(12.7 \pm 2.1) / 10$ & $\mathbf{2 . 9} \pm \mathbf{0 . 7}$ \\
\hline
\end{tabular}

Tabelle 7.9: Ergebnisse für die Quotienten aus Oberflächendiffusion und Temperatur sowie den Parameter $\Delta_{1}$ gemäß Berechnungsmethode 1 bei der Bestrahlung von Si Oberflächen.

\begin{tabular}{|l|l|l|}
\hline & $D_{s} / T\left[10^{-20} \frac{\mathrm{m}^{2} /}{\mathrm{dpa} \cdot \mathrm{K}}\right]$ & $\boldsymbol{\Delta}_{\mathbf{1}}$ \\
\hline$T=300 \mathrm{~K}, \Delta t=1 \mathrm{~ns}$ & $(21.9 \pm 1.0) / 300$ & $\mathbf{4 2} \pm \mathbf{1 2}$ \\
\hline$T=10 \mathrm{~K}, \Delta t=50 \mathrm{ps}$ & $(1.1 \pm 0.1) / 10$ & $\mathbf{3 4} \pm \mathbf{6}$ \\
\hline
\end{tabular}

Tabelle 7.10: Ergebnisse für die Quotienten aus Oberflächendiffusion und Temperatur sowie den Parameter $\Delta_{1}$ gemäß Berechnungsmethode 2 bei der Bestrahlung von Si Oberflächen.

zu einer Verkleinerung von $\Delta_{1}$.

\subsubsection{Viskoses Oberflächenfließen und ballistischer Transport}

Methoden Um die Effekte des viskosen Oberflächenfließens und des ballistischen Transports auf Si Oberflächen gegeneinander abschätzen zu können, wird Gleichung (7.8) herangezogen, durch die genau die Wellenlänge $\lambda_{c}$ bestimmt ist, für die beide Prozesse gleiches Gewicht haben. Für größere Wellenlängen dominiert der ballistische Transport und für kleinere das viskose Oberflächenfließen. Die Berechnung der verschiedenen Parameter erfolgt analog zum Vorgehen beim CuTi System. Auch hier sind schräge Einschüsse mit den Winkeln $\alpha=20^{\circ}, \alpha=45^{\circ}$ und $\alpha=65^{\circ}$ zur Substratnormalen bei den Umgebungstemperaturen $T=300 \mathrm{~K}$ und $T=10 \mathrm{~K}$ durchgeführt worden. Dafür sind beide Methoden, nämlich das sukzessive Bestrahlen einer Oberfläche an verschiedenen Orten sowie das wiederholte Einschießen auf dieselbe Oberfläche an verschiedenen Orten durchgeführt worden. Die Zeit zwischen zwei Einschüssen beträgt ebenfalls 30 ps. Die Randbedingungen, für die im Folgenden die Ergebnisse gezeigt werden, sind die, bei denen die unteren Atomlagen der Probe festgehalten werden und in einigen weiteren Atomlagen die Temperatur der gewünschten Umgebungstemperatur entsprechend geregelt wird. Tests mit einer nicht festgehaltenen Zelle und Temperaturregelung an den periodisch fortgesetzten Rändern zeigen keine signifikant anderen Ergebnisse. Die atomaren Verschiebungen in x-Richtung $\delta$ werden für die ganze Probe, die obere Hälfte sowie die oberen zehn Prozent der Probe berechnet, wobei die Kantenlänge der Probe bei $87.54 \AA$ bzw. $87.70 \AA$ liegt für $T=10 \mathrm{~K}$ bzw. $T=300 \mathrm{~K}$.

Ergebnisse Die Dicke des flüssigen Bereichs, sowie das atomare Volumen und die strahlungsinduzierte Viskosität sind in Tab. 7.8 dargestellt. Als Oberflächenenergie ergeben sich aus der Simulation und Gleichung $(7.9) \tilde{\gamma}=1.36 \mathrm{~J} / \mathrm{m}^{2}$ bei $T=300 \mathrm{~K}$ bzw. 


\begin{tabular}{|l|l|l|l|}
\hline & $\delta\left(20^{\circ}\right)[\AA]$ & $\delta\left(45^{\circ}\right)[\AA]$ & $\delta\left(65^{\circ}\right)[\AA]$ \\
\hline$d=8.8 \AA$ & $-12 \pm 7$ & $-25 \pm 7$ & $-32 \pm 5$ \\
\hline$d=44 \AA$ & $-45 \pm 23$ & $-63 \pm 22$ & $-66 \pm 16$ \\
\hline$d=88 \AA$ & $-44 \pm 28$ & $-63 \pm 28$ & $-64 \pm 21$ \\
\hline
\end{tabular}

Tabelle 7.11: Werte der Summen lateraler Verschiebungen $\delta$ für verschiedene Einschusswinkel und verschiedene Dicken $d$ der analysierten Bereiche als Durchschnittswerte nach jeweils 600 Einschüssen bei $\alpha=45^{\circ}$ und $\alpha=65^{\circ}$ bzw. 850 Einschüssen bei $\alpha=20^{\circ}$ bei einer Umgebungstemperatur von $T=300 \mathrm{~K}$.

\begin{tabular}{|l|l|l|l|}
\hline & $\delta\left(20^{\circ}\right)[\AA]$ & $\delta\left(45^{\circ}\right)[\AA]$ & $\delta\left(65^{\circ}\right)[\AA]$ \\
\hline$d=8.8 \AA$ & $-11 \pm 7$ & $-27 \pm 6$ & $-43 \pm 5$ \\
\hline$d=44 \AA$ & $-29 \pm 18$ & $-65 \pm 14$ & $-73 \pm 9$ \\
\hline$d=88 \AA$ & $-28 \pm 19$ & $-64 \pm 14$ & $-73 \pm 9$ \\
\hline
\end{tabular}

Tabelle 7.12: Werte der Summen lateraler Verschiebungen $\delta$ für verschiedene Einschusswinkel und verschiedene Dicken $d$ der analysierten Bereiche als Durchschnittswerte nach jeweils 300 Einschüssen bei einer Umgebungstemperatur von $T=10 \mathrm{~K}$.

$\tilde{\gamma}=1.52 \mathrm{~J} / \mathrm{m}^{2}$ bei $T=10 \mathrm{~K}$.

Bei $T=300 \mathrm{~K}$ sind für die verschiedenen Winkel jeweils mindestens 600 Einschüsse ausgewertet worden. Die resultierenden Werte für die atomaren Verschiebungen $\delta$ sind in Tab. 7.11 und die entsprechenden Resultate für jeweils 300 Einschüsse bei $T=10 \mathrm{~K}$ sind in Tab. 7.12 dargestellt. Es zeigt sich, dass die Umgebungstemperatur keinen signifikanten Einfluss auf die Summe atomarer Verschiebungen hat. Allerdings ist der Fehler der Ergebnisse bei der höheren Temperatur deutlich größer, obwohl bei der höheren Temperatur bereits mindestens doppelt so viele Einschüsse berücksichtigt worden sind. Die Ergebnisse zeigen des Weiteren, dass es keinen signifikanten Unterschied zwischen den Ergebnissen für $\delta$ gibt, wenn bei der Summation der atomaren Verschiebungen in x-Richtung zum einen die gesamte Probe und zum anderen nur die obere Hälfte berücksichtigt wird. Allerdings sind die Werte für $\delta$ signifikant kleiner (dem Betrage nach), wenn nur die oberen $8.8 \AA$ der Schicht berücksichtigt werden. Deshalb werden die Werte der Übergangswellenlängen in Tab. 7.13 nur für die $\delta$-Werte berechnet, bei denen die obere Probenhälfte oder nur die oberen 10 Prozent der Probe berücksichtigt werden.

Die Werte der Übergangswellenlängen zeigen für beide analysierten Probenbereiche und beide Temperaturen einen Anstieg mit dem Einschusswinkel. Außerdem sind die Übergangswellenlängen bei $T=300 \mathrm{~K}$ etwas kleiner als bei $T=10 \mathrm{~K}$, aber dieser kleine Unterschied bewegt sich innerhalb der Fehlerbalken und ist somit nicht signifikant. Bei der höheren Temperatur sind deutlich mehr Einschüsse nötig, damit die Größe des Fehlers in einem ähnlichen Bereich wie bei der tieferen Temperatur liegt. 


\begin{tabular}{|l|l|l|l|}
\hline & $\lambda_{c}\left(20^{\circ}\right)[\mathrm{nm}]$ & $\lambda_{c}\left(45^{\circ}\right)[\mathrm{nm}]$ & $\lambda_{c}\left(65^{\circ}\right)[\mathrm{nm}]$ \\
\hline$d=8.8 \AA, T=10 \mathrm{~K}$ & $42 \pm 14$ & $44 \pm 6$ & $52 \pm 5$ \\
\hline$d=44 \AA, T=10 \mathrm{~K}$ & $26 \pm 8$ & $28 \pm 4$ & $40 \pm 4$ \\
\hline$d=8.8 \AA, T=300 \mathrm{~K}$ & $35 \pm 11$ & $40 \pm 8$ & $52 \pm 8$ \\
\hline$d=44 \AA, T=300 \mathrm{~K}$ & $18 \pm 5$ & $25 \pm 6$ & $36 \pm 7$ \\
\hline
\end{tabular}

Tabelle 7.13: Übergangswellenlängen $\lambda_{c}$ für verschiedene Einschusswinkel und verschiedene Dicken $d$ der analysierten Bereiche bei Umgebungstemperaturen von $T=10 \mathrm{~K}$ und $T=300 \mathrm{~K}$.

\begin{tabular}{|l|l|l|}
\hline & $Y$ & $\Delta_{3}$ \\
\hline$\alpha=20^{\circ}$ & 0.5 & $8.4 \pm 5.0$ \\
\hline$\alpha=45^{\circ}$ & 1.3 & $2.6 \pm 0.6$ \\
\hline$\alpha=65^{\circ}$ & 2.6 & $0.69 \pm 0.12$ \\
\hline
\end{tabular}

Tabelle 7.14: Sputterausbeute und Verhältnis von ballistischem Glätten zur Aufrauung durch Sputtererosion bei einer Umgebungstemperatur von $T=10 \mathrm{~K}$.

\subsubsection{Vergleich von glättenden und aufrauenden Mechanismen}

Wie beim CuTi kann auch für den Beschuss von Si der einzige in dieser Arbeit diskutierte deterministische Aufrauungsprozess mit dem Glättungsprozess, der dieselbe Strukturabhängigkeit zeigt, verglichen werden. Mit Gleichung (7.10), den aus den Simulationen zum schrägen Beschuss resultierenden Werten für die Sputterausbeuten und den Werten für den ballistischen Transport in Tab. 7.12 ergibt sich das betragsmäßige relative Verhältnis $\Delta_{3}$ beider Effekte für. Die Ergebnisse für $T=10 \mathrm{~K}$ sind in Tab. $7.14 \mathrm{zu}-$ sammengefasst. Für $T=300 \mathrm{~K}$ liefern die Simulationen im Rahmen des Fehlers gleiche Ergebnisse. Auch hier ergibt sich eine Zunahme der Sputterausbeute mit wachsendem Winkel gegenüber der Substratnormalen bei schrägem Einschuss und dementsprechend gewinnt der aufrauende Prozess an Einfluss. Für die experimentell relevanten Steigungswinkel, die in der Regel unterhalb von $45^{\circ}$ liegen, ist das ballistische Glätten stärker als die Aufrauung durch Sputtererosion.

\subsection{Diskussion der Ergebnisse zur Bestrahlung amorpher Si Oberflächen}

\subsubsection{Allgemeine Phänomene und atomare Dynamik}

Mit dem verwendeten Wert für die Glastemperatur ergibt sich beim amorphen Si mit Hilfe der Flüssigkeitsanalyse ein qualitativ anderes Verhalten als beim metallischen Glas $\mathrm{CuTi}$. Mit den verwendeten Kriterien ergeben sich Lebensdauern der flüssigen Bereiche, die zumindest nicht deutlich größer sind als thermische Schwingungsperioden. Während die Lebensdauer des flüssigen Bereichs bei $\tau_{l i q}=276 \pm 22$ fs liegt, haben sich aus Neu- 
tronenstreuexperimenten Phononenspektren für amorphes Silizium ergeben [KSM+ 84], die zeigen, dass der wesentliche Anteil thermischer Schwingungen bei Schwingungsperioden zwischen 60 fs und 420 fs stattfindet. Ganz ähnliche Grenzen für die Perioden thermischer Schwingungen ergeben sich anhand von ab initio Simulationen und auch von Simulationen mit dem Tersoff Potential für amorphes Silizium [VVARV06]. Neben der kurzen Lebensdauer bilden die als flüssig gekennzeichneten Atome keinen zusammenhängenden Bereich. Somit kann man bei der Bestrahlung von amorphem Si nicht unbedingt von einem Aufschmelzen eines lokalen Bereichs im Sinne der kinetischen Energien sprechen. Vielmehr gibt es mehrere verstreute kleine Bereiche mit dem Energiekriterium gemäß flüssigen Atomen. Dieses Ergebnis ist in qualitativer Übereinstimmung mit früheren Untersuchungen zum Beschuss von kristallinem Silizium bei einer etwas höheren Energie von $5 \mathrm{keV}$ im Rahmen klassischer MD-Simulationen [NGA ${ }^{+}$98; RG95]. Insbesondere wird in $\left[\mathrm{NGA}^{+} 98\right]$ diskutiert, dass sich die Bestrahlungskaskade und damit der Aufenthaltsort als flüssig gekennzeichneter Atome auch bei einer Energie von $2 \mathrm{keV}$ in Subkaskaden aufteilt, während das bei Einschüssen der Energie $400 \mathrm{eV}$ nicht zutrifft. Da für die hier untersuchte Energie $1 \mathrm{keV}$ ebenfalls ein Aufspalten der heißen Bereiche und damit auch der Kaskade beobachtet wird, muss die Energiegrenze, ab der eine Aufspaltung stattfindet, zwischen $400 \mathrm{eV}$ und $1 \mathrm{keV}$ liegen.

Für den Beschuss von amorphem Si kann aus der Verteilung von atomaren Verschiebungsweiten prinzipiell nicht auf die Existenz eines Fließverhaltens geschlossen werden (siehe Kap. 7.3.2). Im folgenden Kapitel wird sich aber zeigen, dass es andere eindeutige Hinweise auf ein strahlungsinduziertes Fließen auch für amorphes Silizium gibt.

\subsubsection{Untersuchung der Prozesse im Rahmen von Kontinuumsmodellen}

Dass es auch beim amorphen Silizium ein strahlungsinduziertes Flüssigkeitsverhalten gibt, zeigt sich bei der Methode zur Bestimmung der Viskosität. Während der Bestrahlung wird eine biaxiale Spannung angelegt und die Zelle dehnt sich in den entsprechenden Richtungen mit einer konstanten Dehnungsrate aus, wie es für eine Flüssigkeit zu erwarten ist. Dabei ist die strahlungsinduzierte Viskosität zwar etwas, aber nicht wesentlich, größer als beim CuTi. Damit ist erwiesen, dass amorphes Si durch Bestrahlung das Verhalten einer Flüssigkeit zeigt. Außerdem ist strahlungsinduziertes Fließen in amorphisiertem Silizium zumindest bei Bestrahlungsenergien im MeV Bereich auch experimentell bekannt [Vol91] und auch bei Ionenbestrahlung im niedrigen keV Energiebereich gibt es für den amorphen Halbleiter Germanium Hinweise auf viskoses Fließen [Edl06; EM07]. Die Ausdehnung des flüssigen Bereichs sollte dann auch bei diesem System durch die Ausdehnung des Bereichs, in dem sich Atome, die nach dem verwendeten Kriterium als flüssig bezeichnet werden, befinden, bestimmt sein. Die vertikale Ausdehnung der flüssigen Bereiche an der Oberfläche $a_{z}$ liegt in derselben Größenordnung wie beim metallischen Glas CuTi. Somit ist, wenn auch hier ähnliche Strukturgrößen wie beim experimentell bekannten metallischen Glas $\mathrm{Zr}_{65} \mathrm{Al}_{7.5} \mathrm{Cu}_{27.5}$ betrachtet werden (lateral $20 \mathrm{~nm}$, vertikal $10 \mathrm{~nm}$, siehe Kap. 5.1), das Oberflächenfließen gegenüber dem Volumenfließen 
dominant oder zumindest ein wesentlicher Anteil der Fließprozesse.

Fließen und Oberflächendiffusion Der Vergleich von Oberflächenfließen zu Oberflächendiffusion zeigt bei $T=300 \mathrm{~K}$ deutlich die Dominanz des Oberflächenfließens für beide Methoden zur Berechnung des Glättungsbeitrags der Oberflächendiffusion. Dass bei $T=300 \mathrm{~K}$ der Wert von $\Delta_{1}$ für die zweite Berechnungsmethode von $D_{s} / T$ kleiner ist als bei der ersten Berechnungsmethode, lässt sich dadurch erklären, dass thermische Schwingungen mit in die Berechnung des Diffusionskoeffizienten eingehen. Damit erhöht sich der Wert des Diffusionskoeffizienten, wenn er durch Summation über mehrere Intervalle berechnet wird, weil in jedem Intervall thermische Schwingungen einen Beitrag leisten (siehe hierzu auch entsprechende Diskussion für CuTi in Kap. 7.2.3). Die realistischere Einschätzung der Temperatur sollte dagegen zu einer Verkleinerung von $D_{s} / T$ und damit zu einer Erhöhung von $\Delta_{1}$ bei der zweiten Berechnungsmethode führen. Beim amorphen $\mathrm{Si}$ ist also bei der Umgebungstemperatur $T=300 \mathrm{~K}$ bei der zweiten Berechnungsmethode der Effekt der realistischeren Temperatureinschätzung weniger bedeutend als der Fehler, der durch die wiederholt eingehenden thermischen Schwingungen bei der Berechnung von $D_{s} / T$ als Summe der Ergebnisse aus den verschiedenen Intervallen entsteht. Beim CuTi hatte sich bei $T=300 \mathrm{~K}$ für Berechnungsmethode 2 ein etwas kleinerer Wert als für Methode 1 ergeben (allerdings gleich innerhalb des angegebenen Fehlerbalkens). Das bedeutet, dass dort der durch thermische Schwingungen bedingte Fehler im Verhältnis zu der Verbesserung durch die realistischere Temperatureinschätzung etwas kleiner ist als beim Si. Das entspricht insofern der Erwartung als die absoluten Werte der Diffusionskoeffizienten für CuTi um etwa einen Faktor 10 über denen für Si liegen. Da thermische Schwingungen in beiden Systemen von gleicher Größenordnung sind, ist ihr Anteil beim Si größer als beim CuTi und hat dementsprechend einen größeren Beitrag im Diffusionskoeffizienten.

Bei $T=10 \mathrm{~K}$ zeigt sich dagegen, dass der Effekt der realistischeren Temperatureinschätzung in der zweiten Berechnungsmethode mehr Gewicht hat als der dort entstehende Fehler durch den größeren Beitrag thermischer Schwingungen bei der Summation über die verschiedenen Intervalle. Die zweite Berechnungsmethode liefert hier nämlich für $D_{s} / T$ einen kleineren und somit für $\Delta_{1}$ einen größeren Wert. Der Grund dafür ist, dass bei $T=10 \mathrm{~K}$ der Fehler in Berechnungsmethode 1 sehr viel größer als bei $T=300 \mathrm{~K}$ ist. Wie schon in Kap. 7.2.3 für CuTi diskutiert ist auch für Si bei tiefen Temperaturen Berechnungsmethode 2 wesentlich sinnvoller als Berechnungsmethode 1.

Die in Kap. 7.1.3.2 vorgestellte und für $\mathrm{CuTi}$ auch angewendete Methode 3 zur Berechnung des Beitrags der Oberflächendiffusion zur Glättung ist für Si nicht angewendet worden, weil eine mittlere Diffusionslänge von Adatomen auf einer Oberfläche von amorphem Si nicht bekannt ist. Es finden sich zwar Werte typischer Aktivierungsenergien und Diffusionskoeffizienten für die Diffusion von Adatomen auf kristallinen Si Oberflächen [MKWL91], aber das Diffusionsverhalten auf kristallinen Oberflächen kann von dem auf amorphen Oberflächen stark abweichen. Es besteht der prinzipielle Unterschied zwischen Diffusionsbewegung auf kristallinen und amorphen Oberflächen, dass es für die Kristalloberfläche feste Aktivierungsenergien gibt, während es für amorphe Ober- 
flächen eine Verteilung von Aktivierungsenergien gibt. Das führt dazu, dass sich auf amorphen Oberflächen ein Adatom zunächst bewegt, bis es eine Position erreicht hat, für die die Aktivierungsenergie für einen weiteren Sprung sehr groß ist, so dass ein weiterer Sprung des Adatoms auf den relevanten Zeitskalen sehr unwahrscheinlich ist und somit das Adatom quasi gefangen und damit kein Adatom mehr ist. Deshalb ist es für amorphe Oberflächen auch sinnvoll von einer mittleren Diffusionslänge zu sprechen, während bei kristallinen Oberflächen der Diffusionskoeffizient die relevante Größe ist. Außerdem ist Diffusion auf kristallinen Oberflächen anisotrop [MKWL91] im Gegensatz zur Diffusion auf amorphen Oberflächen. Das Verhalten der Adatomdiffusion auf amorphen Oberflächen ist für metallische Gläser bekannt und die mittlere Diffusionslänge kann aus einer Modellierung der Entwicklung der Oberflächenmorphologie während des Wachstums abgeschätzt werden [RML $\left.{ }^{+} 00\right]$. Das Wachstum amorpher Si Schichten ist ebenfalls untersucht worden [YZWL96], jedoch ist eine ausreichende Modellierung des Wachstums, aus der auf die mittlere Diffusionslänge von Adatomen geschlossen werden kann, nicht bekannt. Bezüglich der kinetischen Aufrauung während des Schichtwachstums durch thermisches Verdampfen zeigen die amorphen Si Schichten [YZWL96] qualitative Ähnlichkeiten im Vergleich zum Wachstum amorpher metallischer Schichten $\left[\mathrm{RML}^{+} 00 ;\right.$ Rai00], so dass ähnliche dominierende Prozesse angenommen werden können, und insbesondere zeigt sich auch beim amorphen Si, dass Oberflächendiffusion eine wesentliche Rolle spielt [YZWL96]. Die Bestimmung einer mittleren Diffusionslänge von Adatomen sollte für Si mit derselben Methode wie für das metallische Glas prinzipiell möglich sein, ist aber bisher noch nicht durchgeführt worden. Somit stehen für Si beim Vergleich von Oberflächendiffusion und Oberflächenfließen zur Bestimmung des Anteils der Oberflächendiffusion bisher nur die Werte zur Verfügung, die sich auf den Zeitskalen der MD-Simulation ergeben. Die Untersuchungen zum metallischen Glas CuTi haben aber gezeigt, dass der Unterschied zwischen den auf der MD-Zeitskala (Methode 2) und den auf experimenteller Zeitskala bestimmten Diffusionskoeffizienten (Methode 3) bei einem Faktor von etwa 2.2 liegt mit dem größeren Wert für den auf experimenteller Zeitskala bestimmten Diffusionskoeffizienten. Falls auch beim Si ein ähnliches Verhältnis zwischen dem mit Methode 2 auf der Zeitskala der Simulation und einem auf experimenteller Zeitskala abgeschätzten Diffusionskoeffizienten bestünde, würde sich ein Wert für $\Delta_{1}$ ergeben, der deutlich größer als 1 ist, denn der Diffusionskoeffizient geht in $\Delta_{1}$ linear ein.

Mit dieser Argumentation ist abschließend klar geworden, dass auch beim amorphen Si das Oberflächenfließen für alle Wellenlängen über die Oberflächendiffusion dominiert, solange Oberflächenfließen ein wesentlicher Bestandteil der gesamten Fließprozesse ist, wie es bei der hier betrachteten Energie zutrifft.

Wäre der Fließprozess im Wesentlichen durch ein Volumenfließen beschrieben, so würde der Vergleich des Volumenfließens mit der Oberflächendiffusion eine Übergangswellenlänge gemäß Gl. (7.12) liefern, oberhalb der das Volumenfließen die Oberflächendiffusion dominiert. Für $T=300 \mathrm{~K}$ ergibt sich mit Berechnungsmethode $1 \mathrm{bzw} .2$ $\lambda_{c}^{V F D}=4.6 \pm 1.0 \mathrm{~nm}$ bzw. $\lambda_{c}^{V F D}=5.2 \pm 1.1 \mathrm{~nm}$. Auch dann wäre also der Fließprozess für die meisten relevanten Strukturgrößen gegenüber der Oberflächendiffusion dominierend. 
Fließen und ballistisches Glätten Die aus der MD-Simulation berechneten Werte für die Oberflächenenergie $\left(1.36 \mathrm{~J} / \mathrm{m}^{2}\right.$ bei $\left.T=300 \mathrm{~K}\right)$ liegen im Bereich der aus der Literatur bekannten Werte, wenngleich erwähnt werden muss, dass die in der Literatur vorhandenen Werte recht stark streuen. So werden in $\left[\mathrm{EWF}^{+}\right.$93] experimentell bestimmte Werte für die freie Oberflächenenergie angegeben, die je nach Kristallorientierung zwischen $1.23 \mathrm{~J} / \mathrm{m}^{2}$ und $1.43 \mathrm{~J} / \mathrm{m}^{2}$ liegen. Aus einer Kombination von klassischer MD-Simulation und ab initio Rechnungen ergibt sich in [HIKS05] eine Oberflächenenergie von $1.05 \pm 0.14 \mathrm{~J} / \mathrm{m}^{2}$, allerdings für höhere Temperaturen als Raumtemperatur. Während in den Simulationen (sowohl der eigenen als auch der zitierten) jeweils die Oberflächenenergie berechnet wird, entspricht der Wert der zitierten experimentellen Arbeit der freien Oberflächenenergie. Ein Vergleich der Daten legt nahe, dass der Entropiebeitrag zur freien Oberflächenenergie sehr klein ist, denn er führt dazu, dass die freie Oberflächenenergie einen kleineren Wert hat als die Oberflächenenergie, und diese Tendenz zeigen die Werte nicht eindeutig.

Für die verwendeten Methoden zur Berechnung des ballistischen Glättungseffekts gelten beim Si dieselben Argumente wie beim $\mathrm{CuTi}$, so dass auch hier die Ergebnisse vorgestellt worden sind, die sich aus der sukzessiven Bestrahlung der Probe ergeben. In qualitativer Übereinstimmung mit den Ergebnissen für $\mathrm{CuTi}$ ergibt sich auch beim $\mathrm{Si}$ nach Summation über die beteiligten Atome eine laterale Verschiebung in Richtung der lateralen Komponente des eingeschossenen Atoms. Wie schon beim CuTi äußert sich auch hier die rein ballistische Natur des Effekts der lateralen Verschiebung darin, dass die Ergebnisse für die zwei unterschiedlichen Umgebungstemperaturen nicht signifikant voneinander abweichen. Es sind bei diesem Effekt somit keine thermischen Prozesse beteiligt. Ebenfalls in Übereinstimmung mit den Ergebnissen zum CuTi ist der Fehler bei $T=300 \mathrm{~K}$ deutlich größer als bei $T=10 \mathrm{~K}$. Dieser Einfluss der Temperatur auf die Größe des Fehlers lässt sich dadurch erklären, dass thermische Schwingungen in wesentlichem Maß den Fehler bedingen. Für die Werte von $\delta$ ergibt sich kein Unterschied, wenn einerseits die gesamte Probe und andererseits nur die obere Hälfte der Probe berücksichtigt wird. Das bedeutet, dass in der unteren Probenhälfte keine gerichteten ballistischen Prozesse stattfinden. Allerdings ist der Unterschied zwischen diesen Ergebnissen und denen, die sich bei Berücksichtigung der oberen 10 Prozent der Probe ergeben, größer als es beim CuTi der Fall ist. Diese Tendenz stimmt mit der Beobachtung aus den Simulationsdaten überein, dass das eingeschossene Atom im Si tiefer eindringen kann als beim CuTi. Dieser Unterschied lässt sich durch die porösere Struktur des amorphen Siliziums erklären.

Die Werte für die Summen lateraler Verschiebungen bei $20^{\circ}$ Einschusswinkel sind größer als beim Beschuss von amorphem Kohlenstoff mit einer Energie von $100 \mathrm{eV}$ $\left[\mathrm{MGC}^{+} 05\right]$, wobei wie in Kap. 7.1.3.4 erläutert $\delta \cos (\alpha)$ den $\delta$-Werten aus $\left[\mathrm{MGC}^{+} 05\right]$ entspricht. Im zitierten Artikel wird der Effekt des ballistischen Glättens auch für mit dem Tersoff Potential modelliertes Si untersucht und es ergibt sich ein ähnlicher Wert wie beim Kohlenstoff, nämlich in der Notation dieser Arbeit $\frac{\delta \cdot \cos (\alpha)}{\tan (\alpha)}=2.7 \pm 0.2 \mathrm{~nm}$ für Steigungswinkel $\alpha<20^{\circ}$. Für die Einschussenergie von $1 \mathrm{keV}$ ergeben sich dagegen für $20^{\circ}, 45^{\circ}$ und $65^{\circ}$ die Werte $11.6 \pm 5.9 \mathrm{~nm}, 4.5 \pm 1.6 \mathrm{~nm}$ und $1.3 \pm 0.3 \mathrm{~nm}$. Da in $\left[\mathrm{MGC}^{+} 05\right]$ nur die Winkel $\alpha \leq 20^{\circ}$ untersucht wurden, kann hier nur das Ergebnis für 
$20^{\circ}$ verglichen werden. Der deutlich größere Wert in dieser Arbeit ist auf die höhere Bestrahlungsenergie zurückzuführen, weil ansonsten gleiche Systeme verwendet worden sind. Eine in $\left[\mathrm{MGC}^{+} 05\right]$ diskutierte Sättigung des Effekts ab einer Einschussenergie von $120 \mathrm{eV}$ kann damit nicht bestätigt werden. In dieser Arbeit sind die Fehler deutlich größer, weil eine deutlich größere Anzahl von Atomen bei jedem Einschuss berücksichtigt werden muss. Dass der Fehler bei $20^{\circ}$ in dieser Arbeit so groß ist, kann dadurch gemildert werden, dass sich bei $T=10 \mathrm{~K}$ ähnliche Ergebnisse ergeben. Anders als in $\left[\mathrm{MGC}^{+} 05\right.$ ] für Winkel $\alpha \leq 20^{\circ}$ ist in dieser Arbeit im Winkelbereich $20^{\circ} \leq \alpha \leq 65^{\circ}$ der Ausdruck $\frac{\delta \cdot \cos (\alpha)}{\tan (\alpha)}$ als Funktion des Winkels nicht konstant. Dieselbe Beobachtung wurde auch für das metallische System in Kap. 7.1.3.5 gemacht. Das spricht dafür, dass sich bei den größeren Winkeln der Mechanismus des ballistischen Glättens ändert. Dass der Ausdruck $\frac{\delta \cdot \cos (\alpha)}{\tan (\alpha)}$ nicht bis zu beliebig großen Winkeln konstant bleiben kann, ist schon deshalb einleuchtend, weil sonst im Grenzfall streifenden Einschusses $\left(\alpha \rightarrow 90^{\circ}\right) \delta$ divergieren müsste, und das ist bei einer begrenzten Einschussenergie nicht möglich. Für Si scheint der Wert von $\delta$ bereits zwischen $45^{\circ}$ und $65^{\circ}$ zu sättigen.

Die Werte der Übergangswellenlängen sind kleiner als beim zuvor untersuchten CuTi. Daraus kann gefolgert werden, dass beim porösen Si der ballistische Glättungseffekt im Vergleich zum Oberflächenfließen eine etwas größere Rolle spielt, als es beim CuTi der Fall ist. Die berechneten Übergangswellenlängen gelten für den bei der untersuchten Bestrahlungsenergie relevanten Fall, dass Oberflächenfließen der dominierende Fließprozess ist.

Falls das Volumenfließen der dominierende Fließprozess wäre, würden sich mit Hilfe von Gl. (7.15) entsprechende Übergangswellenlängen ergeben, oberhalb derer der Fließprozess dominieren würde. Die entsprechenden Werte lauten $\lambda_{c}^{V F}\left(20^{\circ}\right)=18 \pm 10 \mathrm{~nm}$, $\lambda_{c}^{V F}\left(45^{\circ}\right)=9.5 \pm 3.4 \mathrm{~nm}$ und $\lambda_{c}^{V F}\left(65^{\circ}\right)=4.6 \pm 1.2 \mathrm{~nm}$. Beide Prozesse wären somit von Bedeutung, wobei das Volumenfließen für den Großteil der relevanten Strukturgrößen dominiert.

Vergleich glättender und aufrauender Mechanismen Ebenso wie bei den CuTi Oberflächen ist auch beim Si die erwartete Zunahme der Sputterausbeute mit dem Einschusswinkel zu beobachten. Die Ergebnisse stimmen damit auch hier mit den Erwartungen [Sig69] überein. Da im experimentell relevanten Bereich von Steigungswinkeln bereits das ballistische Glätten das Aufrauen durch Sputtererosion überwiegt, sollte mit den außerdem diskutierten Glättungseffekten eine strukturierte amorphe Si Oberfläche insgesamt geglättet werden, solange kein stochastischer Rauschprozess oder ein bisher nicht berücksichtigter Aufrauungsprozess dominiert.

\subsection{Zusammenfassende Diskussion}

Insgesamt hat dieses Kapitel gezeigt, dass für beide untersuchten Materialien der Prozess des Oberflächenfließens den der Oberflächendiffusion dominiert und somit als der physikalische Prozess angesehen werden muss, der die Glättung einer Oberfläche gemäß $\partial h / \partial t \propto-\nabla^{4} h$ beschreibt. Dass dieses Resultat für zwei amorphe Systeme, die sehr 
unterschiedlichen Materialklassen zuzuordnen sind, gültig ist, legt nahe, dass es auch für eine Vielzahl weiterer Systeme zu erwarten ist. Auch der Vergleich von Oberflächenfließen und ballistischem Glätten zeigt für beide Systeme zumindest qualitativ gleiche Ergebnisse. Lediglich der genaue Wert der Übergangswellenlängen unterscheidet sich. Somit zeigen insgesamt die Ergebnisse, die sich aus der Kombination von Kontinuumsmodellen und MD-Simulationen ergeben, ähnliche Resultate für beide Materialsysteme. Dies steht nicht im Widerspruch dazu, dass die mikroskopische Struktur des bestrahlten Bereichs für beide Materialien unterschiedlich ist, wie die Verteilung flüssiger Atome es zeigt, denn die Kontinuumsbeschreibung findet auf einer Skala statt, die oberhalb der Atomgröße liegt. Somit ist zu erwarten, dass das Verhältnis der drei untersuchten Prozesse für viele amorphe Systeme sehr ähnlich ist, denn selbst die gewählten Extrembeispiele (starkes, kovalent gebundenes Glas Si bzw. fragiles metallisches Glas CuTi ohne kovalente Bindungsanteile) zeigen ähnliches Verhalten.

Die in diesem Kapitel gewonnenen Ergebnisse können zur Interpretation der experimentellen Ergebnisse herangezogen werden. Des Weiteren werden sie im Kapitel über numerische Lösungen von Kontinuumsmodellen für eine quantitative Abschätzung der Koeffizienten genutzt.

Abgesehen davon ist die quantitative Abgrenzung verschiedener Glättungseffekte für realistische Modellierungen mit Monte Carlo Simulationen hilfreich, da dort die Mechanismen als Voraussetzung in den Algorithmus eingehen. 


\section{Ergebnisse der Simulationen von Kontinuumsmodellen}

In diesem Kapitel werden die Ergebnisse zu numerischen Lösungen der in Kap. 4 vorgestellten Modelle gezeigt. Die resultierenden Ergebnisse, die durch die Höhenfunktion $h(\vec{x}, t)$ gegeben sind, werden in gleicher Weise wie die experimentell bestimmten STM Bilder dargestellt und lassen sich somit direkt vergleichen. Auch hier ist die Farbskala derart gewählt, dass der tiefste Punkt schwarz und der höchste Punkt weiß erscheint. Die Auswertungen der Höhenfunktionen erfolgen ebenso nach den in Kap. 2.3.4 vorgestellten Methoden.

Zunächst wird das Wachsen einer Schicht der Dicke $d=480 \mathrm{~nm}$, die mit der nominellen Dicke der experimentell hergestellten Schicht identisch ist, simuliert. Dies geschieht mit der in Kap. 4.4 vorgestellten Gleichung (4.13) und einer konstanten Höhenfunktion $h=0$ als Startkonfiguration. Die Parameter $a_{i}$ und $R$ entsprechen denen aus $\left[\mathrm{RML}^{+} 00\right]$ und sind in Tab. 8.1 wiedergegeben. Damit ergibt sich die in Abb. 8.1.a gezeigte Struktur, die der entsprechenden STM-Topographie aus Abb. 5.1, die hier zum Vergleich in Abb. 8.1.b erneut dargestellt ist, sehr ähnlich ist. Insbesondere sind horizontale und vertikale Strukturgröße (also Hügeldurchmesser und Hügelhöhe) und damit verbunden auch die Rauigkeit in guter Übereinstimmung $\left(\sigma_{\mathrm{STM}}=1.78 \pm 0.03 \mathrm{~nm}, \sigma_{\text {Simulation }}=1.77 \mathrm{~nm}\right)$ und außerdem in Übereinstimmung zu früheren Ergebnissen [RML $\left.{ }^{+} 00\right]$. Diese Struktur wird im weiteren Verlauf dieses Kapitels als Ausgangspunkt für die Untersuchung der Auswirkungen strahlungsinduzierter Prozesse verwendet, falls nicht anders erwähnt.

Im Folgenden werden Ergebnisse zu den verschiedenen Kombinationen der in Kap. 4 besprochenen Terme vorgestellt. Falls nicht anders beschrieben ist ein Rauschprozess miteinbezogen. Dabei werden die Ergebnisse aus Kap. 7 berücksichtigt, die quantitative Abschätzungen für den relativen Beitrag der verschiedenen Prozesse liefern. Die Kombinationen verschiedener Prozesse liefern Evolutionsgleichungen, die auf die Ausgangsstruktur aus Abb. 8.1.a angewendet werden. Falls die Verhältnisse der einzelnen Terme nicht quantitativ bekannt sind, werden qualitative Simulationen durchgeführt, in denen

die Koeffizienten in der Evolutionsgleichung nicht quantitativ physikalisch begründet sind.

Die Ergebnisse der simulierten Modelle werden mit den experimentellen Ergebnissen aus Kap. 5 verglichen. Dabei werden insbesondere die Entwicklungen der Oberflächentopographie (Abb. 5.1 - 5.5), der Rauigkeit (Abb. 5.7) und der spektralen Leistungsdichten (Abb. 5.11) aus den experimentellen Untersuchungen mit den entsprechenden Simulationsergebnissen verglichen.

Es werden in den folgenden Abschnitten sowohl Modelle gezeigt, die die experimentellen Ergebnisse relativ gut wiedergeben als auch solche, die die experimentellen Ergebnisse 


\begin{tabular}{|l|l|l|l|l|}
\hline$a_{1}\left[\mathrm{~nm}^{2} / \mathrm{s}\right]$ & $a_{2}\left[\mathrm{~nm}^{4} / \mathrm{s}\right]$ & $a_{3}\left[\mathrm{~nm}^{3} / \mathrm{s}\right]$ & $a_{4}[\mathrm{~nm} / \mathrm{s}]$ & $R\left[\mathrm{~nm}^{4} / \mathrm{s}\right]$ \\
\hline-0.0826 & -0.319 & -0.10 & 0.055 & 0.0174 \\
\hline
\end{tabular}

Tabelle 8.1: Parameter zur Simulation des Schichtwachstums gemäß Gleichung (4.13) nach $\left[\mathrm{RML}^{+} 00\right]$.

a.)

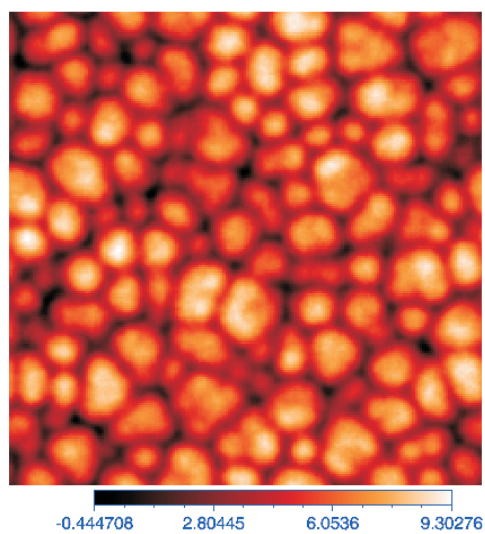

b.)

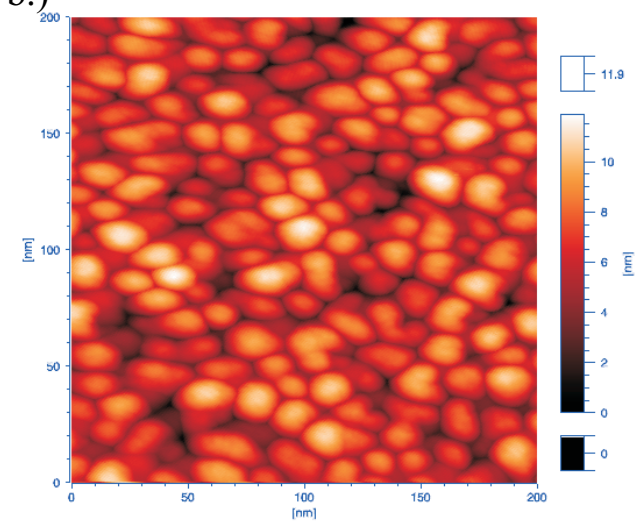

Abbildung 8.1: a.) Topographie der gemäß $\left[\mathrm{RML}^{+} 00\right]$ simulierten gewachsenen $480 \mathrm{~nm}$ dicken Schicht, b.) STM-Aufnahme der unbestrahlten $480 \mathrm{~nm}$ dicken Schicht aus Abb. 5.1 (Bildgröße: $L=200 \mathrm{~nm}$, Einheit der Höhenskala: nm).

nicht wiedergeben und somit ausgeschlossen werden können.

Für die numerische Lösung der Wachstumsgleichung hat sich für die Ortsauflösung $\Delta x=1 \mathrm{~nm}$ eine Zeitschrittweite von $\Delta t=0.001 \mathrm{~s}$ als hinreichend klein erwiesen, um mit dem Integrationsverfahren die richtige Lösung zu erreichen $\left[\mathrm{RML}^{+} 00\right]$. Für die im Folgenden gelösten Gleichungen sind in der Regel dieselben Werte für $\Delta x$ und $\Delta t$ verwendet worden, falls es nicht anders erwähnt wird. Falls Terme enthalten sind, die in der Wachstumsgleichung nicht auftreten, ist jeweils verifiziert worden, dass mit kleinerer Zeitschrittweite dieselben Ergebnisse erreicht werden. Die Größe der simulierten Bildausschnitte beträgt $200 \mathrm{~nm} \times 200 \mathrm{~nm}$, falls nicht anders angegeben.

\subsection{Oberflächenfließen und Sputtererosion}

Modell Zunächst wird simuliert, wie sich die Oberfläche entwickelt, wenn für die die Bestrahlung beschreibende Evolutionsgleichung angenommen wird, dass ein Glättungsprozess durch Oberflächenfließen sowie Aufrauung durch krümmungsabhängige Sputtererosion und stochastisches Rauschen stattfindet. Mathematisch entspricht das der Theorie nach Bradley und Harper [BH88] mit dem Unterschied, dass nach den Ergebnissen aus Kap. 7 bekannt ist, dass der zu $\nabla^{4} h$ proportionale Term Oberflächenfließen 
a.)

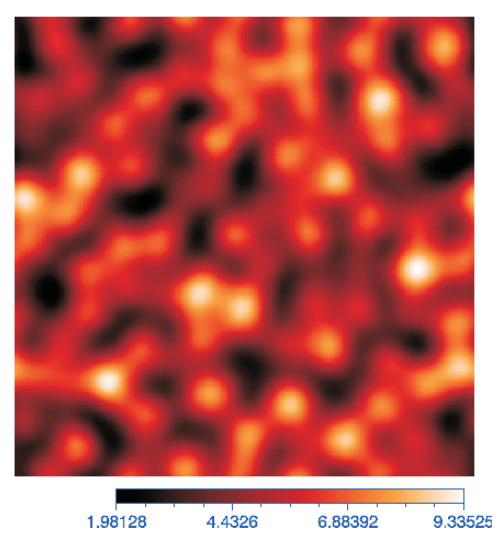

b.)

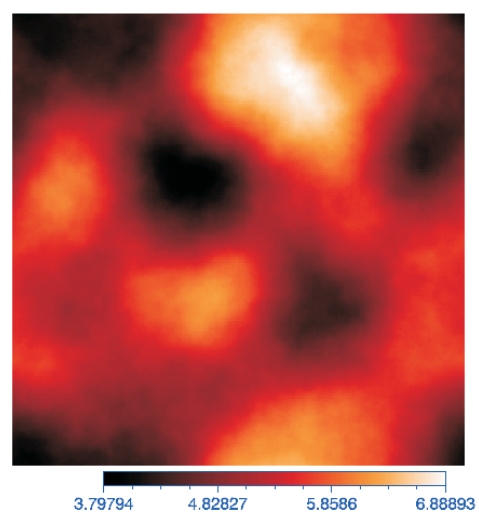

Abbildung 8.2: Topographie der gemäß Gleichung (8.1) simulierten Bestrahlung für Zeiten von a.) $5 \mathrm{~s}$ und b.) $1000 \mathrm{~s}$ (Bildgröße: $L=200 \mathrm{~nm}$, Einheit der Höhenskala: nm).

und nicht Oberflächendiffusion beschreibt. Die mathematische Form ist demnach

$$
\frac{\partial h}{\partial t}=c_{2} \nabla^{2} h+c_{4} \nabla^{4} h+\xi
$$

Zunächst sollen qualitative Ergebnisse gezeigt werden, da bereits unabhängig von den quantitativen Werten gezeigt werden kann, dass dieses Modell die experimentellen Ergebnisse nicht vollständig beschreiben kann. Die hier verwendeten Koeffizienten lauten $c_{2}=-0.1 \mathrm{~nm}^{2} / \mathrm{s}, c_{4}=-22 \mathrm{~nm}^{4} / \mathrm{s}$ und für die Rauschstärke gilt $R=0.02 \mathrm{~nm}^{4} / \mathrm{s}$.

Ergebnisse Die Entwicklung der Oberflächentopographie ist anhand von zwei Beispielen in Abb. 8.2 dargestellt. Als Funktion der Bestrahlungszeit ist die rms-Rauigkeit für die Struktur ausgewertet worden und ebenso sind für einige Bestrahlungszeiten die spektralen Leistungsdichten berechnet worden. Die entsprechenden Resultate finden sich in Abb. 8.3.

Der Vergleich mit den experimentellen Ergebnissen zeigt an Gemeinsamkeiten, dass es einen deutlichen Glättungseffekt gibt, der in beiden Fällen nach einem Potenzgesetz verläuft; qualitativ ähnlich vergrößern sich die Strukturen lateral und werden dabei flacher. In den spektralen Leistungsdichten nehmen in beiden Fällen die Intensitäten in dem Bereich, in dem die ursprünglich vorherrschende Strukturgröße liegt, ab. Allerdings zeigen sich quantitative Unterschiede. So ist der Exponent $\beta$, der den Zerfall der Rauigkeit beschreibt, im Experiment betragsmäßig größer. Andererseits ist der Exponent $\zeta$, der den Zerfall der spektralen Leistungsdichte im Bereich großer $q$-Werte beschreibt, im Experiment betragsmäßig kleiner. Die Ergebnisse ändern sich bezüglich der Rauigkeitsentwicklung kaum, wenn auf den Rauschprozess ganz verzichtet wird (dann gilt: $\beta=-0.180 \pm 0.001)$. Die spektralen Leistungsdichten ändern ihren Verlauf deutlich, wenn kein Rauschterm vorhanden ist. Anhand von Gleichung (4.11) wird klar, dass kein stationärer Grenzfall existiert und insbesondere für große $q$-Werte die Leistungsdichte 
a.)

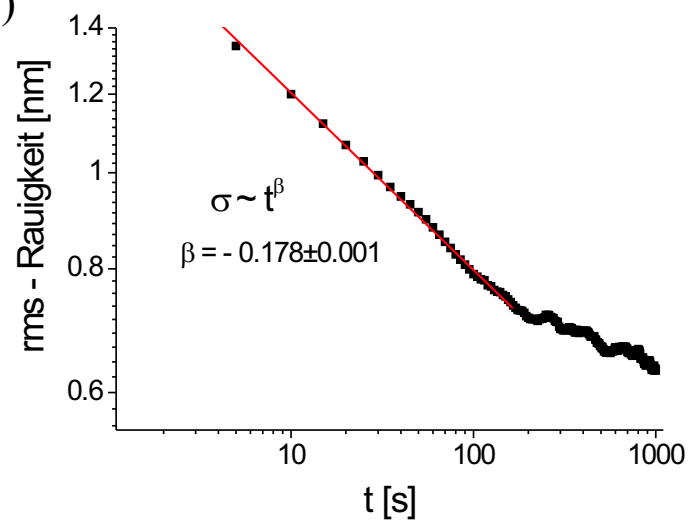

b.)

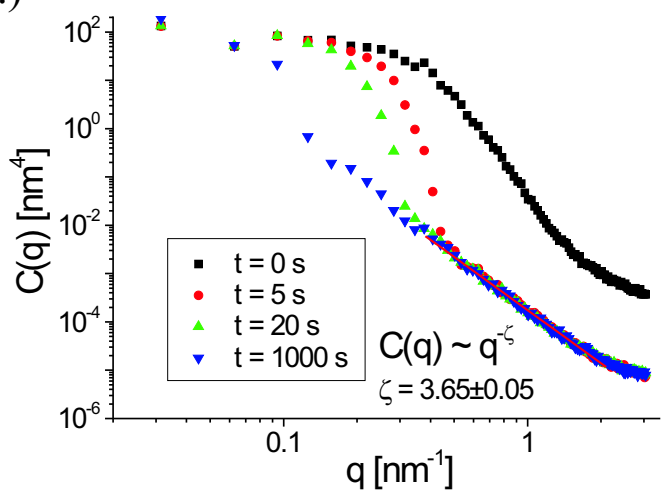

Abbildung 8.3: a.) Entwicklung der rms-Rauigkeit als Funktion der Bestrahlungszeit und b.) spektrale Leistungsdichten nach verschiedenen Bestrahlungszeiten; jeweils für das Modell aus Kap. 8.1.

gegen null geht. Genau dieses Verhalten zeigen Simulationen des Modells ohne einen Rauschprozess.

Diskussion Insgesamt wird damit deutlich, dass bei der Kombination von einem glättenden $\nabla^{4} h$ und einem aufrauenden $\nabla^{2} h$ Term gemäß der Theorie von Bradley und Harper [BH88] mit den gewählten Koeffizienten die Rauigkeit nicht so stark abfällt wie im Experiment. Um die Modellierung dem Experiment anzupassen, müsste daher der Beitrag des glättenden $\nabla^{4} h$ Terms relativ zum aufrauenden $\nabla^{2} h$ Term erhöht werden. Dann würde sich aber der Zerfall der spektralen Leistungsdichte noch mehr einem $q^{-4}$ Verhalten nähern und sich somit weiter vom experimentellen Ergebnis entfernen. Damit ist insgesamt erwiesen, dass die experimentell beobachtete Strukturentwicklung nicht durch die in diesem Abschnitt besprochene Gleichung beschrieben werden kann.

\subsection{Oberflächenfließen und ballistisches Glätten}

Modell In diesem Kapitel werden die Ergebnisse aus Kap. 7 verwendet, um die Glättungsprozesse quantitativ so zu beschreiben, wie es die Ergebnisse aus den MDSimulationen nahelegen. Demnach trägt sowohl ein glättender $\nabla^{4} h$ Term als auch ein glättender $\nabla^{2} h$ Term bei. In Kap. 7 konnte gezeigt werden, dass der $\nabla^{4} h$ Term auf viskosem Fließen im Oberflächenbereich beruht und dass der Beitrag dieses Fließprozesses um ein Vielfaches größer ist als der Beitrag der Oberflächendiffusion, der ebenfalls durch einen glättenden $\nabla^{4} h$ Term beschrieben wird. Beide Beiträge sollten addiert werden, um den gesamten Glättungsbeitrag proportional zu $\nabla^{4} h$ zu erhalten. Weil der Beitrag des Fließterms aber um etwa zwei Größenordnungen größer ist, ist es hinreichend genau, nur den Fließprozess zu berücksichtigen. Unter der Annahme, dass kleine Steigungswinkel $\left(\alpha(=\arctan (|\nabla h|))<20^{\circ}\right)$ vorherrschen, konnte in Kap. 7 gezeigt werden, dass 


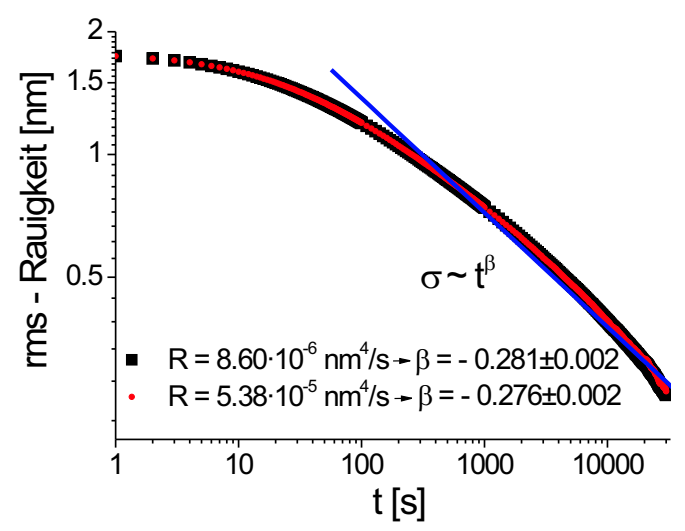

Abbildung 8.4: Entwicklung der rms-Rauigkeit als Funktion der Bestrahlungszeit für zwei verschiedene Rauschstärken im Modell aus Kap. 8.2 (Schwarze und rote Symbole liegen nahezu übereinander.).

bezüglich des $\nabla^{2} h$ Terms der glättende Beitrag des ballistischen Transports wesentlich größer als der aufrauende Beitrag der Sputtererosion ist. Auch hier müssten die Koeffizienten beider Beiträge addiert werden. Da der Glättungsbeitrag wesentlich größer als der der Aufrauung ist, wird der aufrauende Beitrag vernachlässigt. Zusammengefasst ist wieder eine Gleichung der Form

$$
\frac{\partial h}{\partial t}=c_{2} \nabla^{2} h+c_{4} \nabla^{4} h+\xi
$$

zu simulieren, wobei für die Parameter gilt:

$$
\begin{aligned}
2 R & =F Y \Omega^{2} \quad(\text { entspricht Gl. (4.31)) } \\
c_{2} & =F \Omega\left|\frac{\delta}{\tan (\alpha)}\right|=\frac{2 R\left|\frac{\delta}{\tan (\alpha)}\right|}{Y \Omega} \text { (nach Gl. (4.23) und Gl. (4.31)) } \\
c_{4} & =-\frac{\gamma a_{z}^{3}}{3 \eta}=-\left(\frac{\lambda_{c}}{2 \pi}\right)^{2} \cdot c_{2} \quad \text { (nach Gl. (4.26) und Gl. (7.8)) } .
\end{aligned}
$$

Mit einem durchschnittlich im Experiment verwendeten Fluss einfallender Ionen von $F=2.5 \cdot 10^{12} \frac{1}{\mathrm{~cm}^{2} \cdot \mathrm{s}}$ (entspricht $0.4 \mu \mathrm{A} / \mathrm{cm}^{2}$, siehe Kap. 5), der für einen mittleren Steigungswinkel von $20^{\circ}$ bestimmten Sputterausbeute $Y=3.2$ aus Tab. 7.7 und dem mittleren atomaren Volumen $\Omega=14.66 \AA^{3}$ ergeben sich als Parameter der Gleichung (8.2) $R=8.60 \cdot 10^{-6} \mathrm{~nm}^{4} / \mathrm{s}, c_{2}=8.06 \cdot 10^{-3} \mathrm{~nm}^{2} / \mathrm{s}$ und $c_{4}=-0.810 \mathrm{~nm}^{4} / \mathrm{s}$.

Ergebnisse Für diese Gleichung zeigt die numerische Lösung, dass die Rauigkeit für große Werte der Zeit einem Potenzgesetz folgend abnimmt (siehe Abb. 8.4). Der zugehörige Exponent $\beta$ für den Zeitbereich, in dem die wesentliche Abnahme der Rauigkeit stattfindet, liegt mit seinem Wert $\beta=-0.281 \pm 0.002 \mathrm{~nm}$ deutlich näher am 
a.)

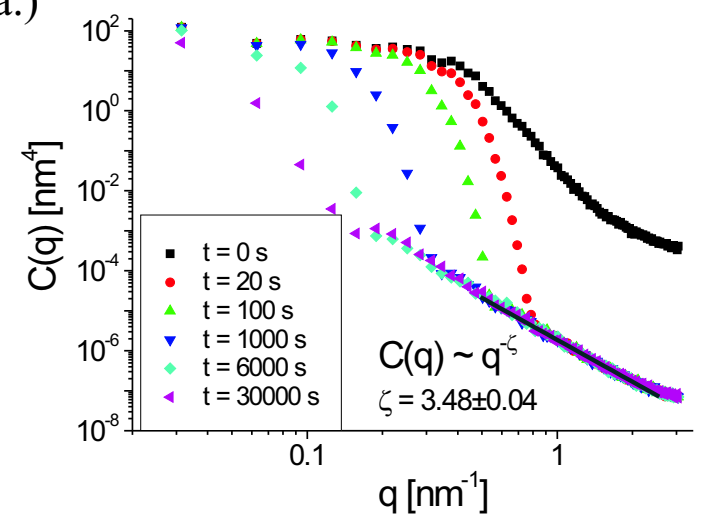

b.)

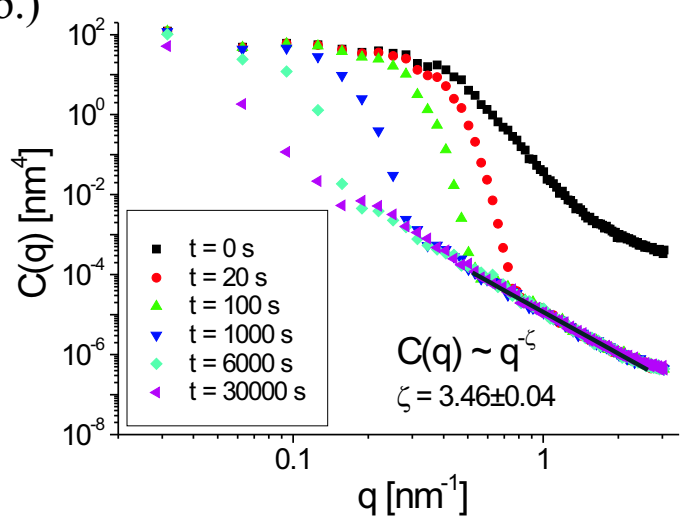

Abbildung 8.5: Spektrale Leistungsdichten nach verschiedenen Bestrahlungszeiten im Modell aus Kap. 8.2 für a.) $R=8.60 \cdot 10^{-6} \mathrm{~nm}^{4} / \mathrm{s}$ und b.) $R=5.38$. $10^{-5} \mathrm{~nm}^{4} / \mathrm{s}$.

experimentellen Resultat als der im vorigen Kapitel erhaltene. Für den gesamten Zeitbereich lässt sich allerdings nicht ein Potenzgesetz mit einem Exponenten anpassen. Des Weiteren zeigt die Analyse der Daten, dass auch ein exponentieller Abfall nicht den Daten entspricht. Die Näherung durch das Potenzgesetz im Bereich des hauptsächlichen Zerfalls der Rauigkeit entspricht dem Bereich, aus dem fast alle experimentellen Daten in Abb. 5.7 stammen und ebenfalls durch ein Potenzgesetz beschrieben werden können. Die in Abb. 8.5.a dargestellten spektralen Leistungsdichten zeigen in ihrem Verlauf für große $q$-Werte einen Zerfall gemäß $C(q) \propto q^{-\zeta}$. Dabei ist der Wert des Exponenten $\zeta$ etwas kleiner als im vorigen Abschnitt und damit näher am experimentellen Ergebnis. Übereinstimmend mit den experimentellen Ergebnissen ist erkennbar, dass die Intensität im Spektrum für die Größen, die der ursprünglich vorherrschenden Strukturgröße entsprechen, am stärksten abnimmt. Mit fortschreitender Zeit dehnt sich dieser Bereich zu kleineren $q$-Werten, entsprechend größeren Strukturen, aus.

Auffällig ist allerdings, dass im Bereich großer $q$-Werte die Intensität der spektralen Leistungsdichten im Vergleich zu der durch die Wachstumsgleichung hergestellten Struktur deutlich kleiner ist. Eine solch starke Abnahme der Intensität in diesem Bereich ist im Experiment nicht beobachtet worden (vgl. Abb. 5.10).

Diskussion Ein Grund für die im Vergleich zum Experiment zu starke Abnahme der Intensität im Bereich großer $q$-Werte könnte sein, dass die Rauschstärke zu klein angenommen worden ist. Diese Begründung ist durch die Form der Gleichung (4.11) gerechtfertigt, denn sie zeigt, dass im Grenzfall kleiner $q$-Werte entsprechend lange Zeiten nötig sind, um die ursprüngliche Struktur zu entfernen. In diesem Bereich dominiert somit der erste Summand in Gleichung (4.11) die spektrale Leistungsdichte und die für die Bestrahlung angenommene Rauschstärke hat fast keinen Einfluss. Im Bereich großer $q$-Werte wird dagegen die ursprüngliche Struktur sehr schnell vernichtet und dementspre- 
$\mathrm{t}=0 \mathrm{~s}$

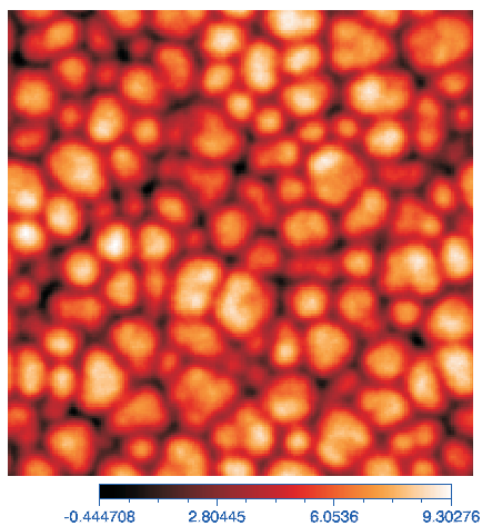

$\mathrm{t}=1000 \mathrm{~s}$

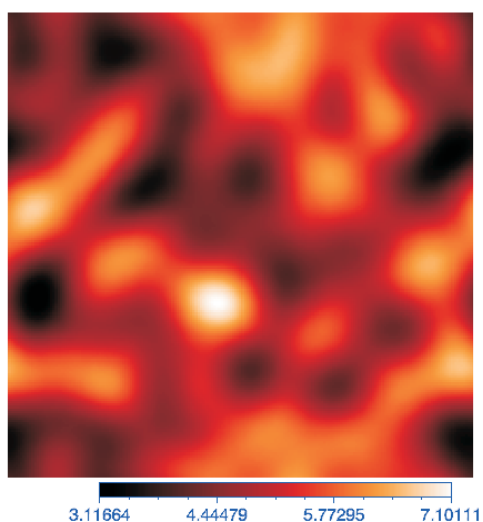

$\mathrm{t}=20 \mathrm{~s}$

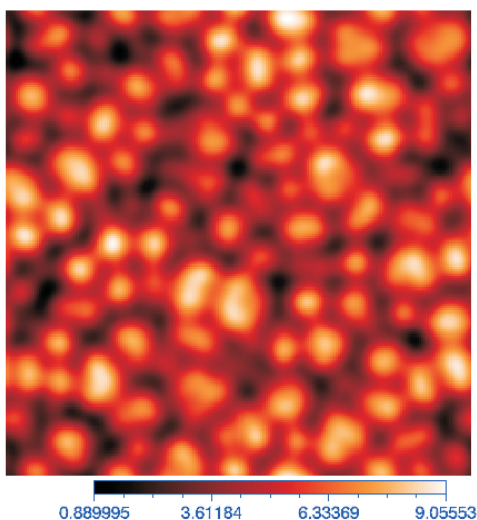

$t=6000 s$

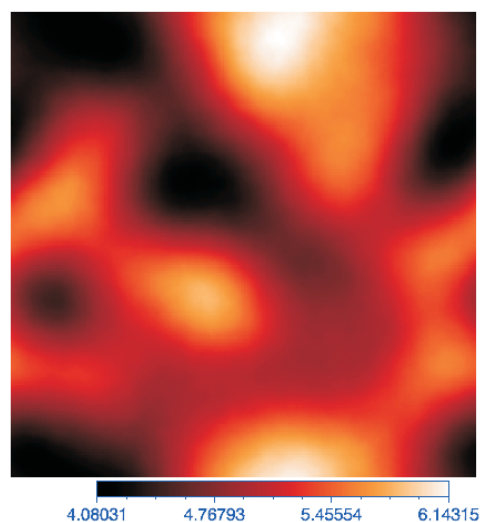

$\mathrm{t}=100 \mathrm{~s}$

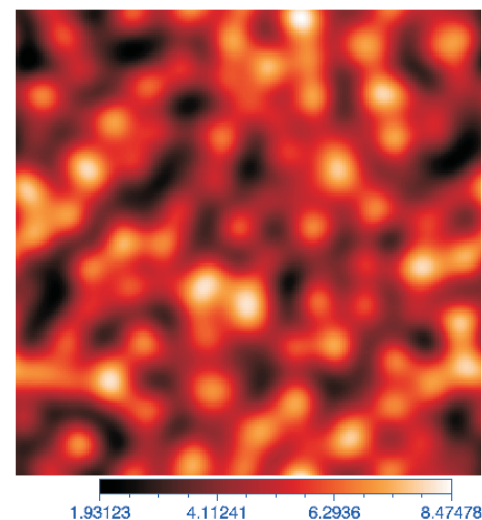

$\mathrm{t}=30000 \mathrm{~s}$

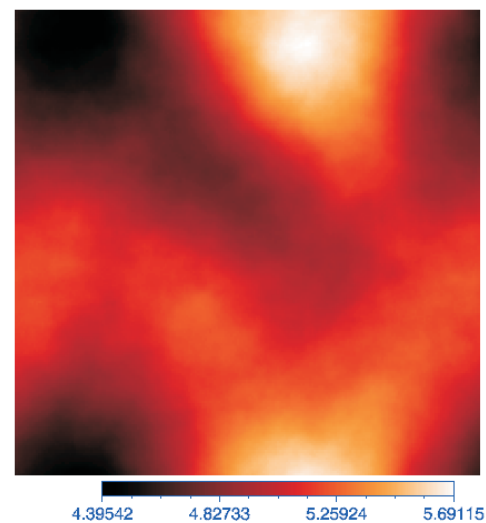

Abbildung 8.6: Entwicklung der Oberflächentopographie als Funktion der Bestrahlungszeit für $R=5.38 \cdot 10^{-5} \mathrm{~nm}^{4} / \mathrm{s}$ im Modell aus Kap. 8.2 (Bildgröße: $L=200 \mathrm{~nm}$, Einheit der Höhenskala: nm).

chend dominiert der zweite Summand in Gleichung (4.11), so dass die für die Bestrahlung angenommene Rauschstärke wesentlichen Einfluss hat.

Neben der Aufrauung durch stochastisches Entfernen von Atomen im Rahmen von Sputterprozessen, ist denkbar, dass auch durch die Produktion von Adatomen, die auf die Oberfläche angehoben werden, vor dem Ablauf eventueller Relaxationsprozesse zunächst ebenfalls ein stochastischer Rauigkeitsbeitrag entsteht. Dieser sollte ebenfalls durch Gleichung (4.31) beschrieben werden, wobei $Y$ dann der Anzahl erzeugter Adatome entspricht. Um beide auf atomarer Skala aufrauenden Prozesse zu berücksichtigen, muss $Y$ der Summe aus pro Einschuss gesputterten Atomen und erzeugten Adatomen entsprechen. Wiederum mit den Ergebnissen aus Kap. 7 für die Anzahl erzeugter Adatome und die Sputterausbeute ergibt sich $Y \approx 20$ und damit als Rauschstärke $R=5.38 \cdot 10^{-5} \mathrm{~nm}^{4} / \mathrm{s}$. Mit unveränderten Koeffizienten $c_{2}$ und $c_{4}$ resultieren die in Abb. 8.4 gezeigte Entwicklung der Rauigkeit und die spektralen Leistungsdichten in Abb. 8.5.b.

Die Entwicklung der Rauigkeit wird durch die Erhöhung der Rauschstärke bis auf kleine Abweichungen bei großen Zeiten nicht sichtbar verändert. Die Rauigkeit ist nur 
unwesentlich größer. Der zugehörige Exponent $\beta$ für den Zeitbereich, in dem die wesentliche Abnahme der Rauigkeit stattfindet, liegt mit seinem Wert $\beta=-0.276 \pm 0.002 \mathrm{~nm}$ sehr dicht am experimentell bestimmten Wert. Somit ist dieses Modell dem Experiment deutlich näher als das im vorigen Kapitel 8.1 untersuchte. In den spektralen Leistungsdichten führt die Erhöhung der Rauschstärke zu höheren Werten der Intensität im Bereich großer $q$-Werte. Jedoch ergibt sich im Bereich großer q-Werte durch die Simulation der Bestrahlung mit erhöhter Rauschstärke immer noch eine stärkere Reduktion der Intensität gegenüber der durch das Wachstum bedingten Struktur, als es im Experiment beobachtet wird (vgl. Abb. 5.10). Mit der größeren Rauschstärke sind die Simulationsergebnisse aber etwas näher an den experimentellen Ergebnissen. Die Entwicklung der Oberflächentopographie für die Simulation mit erhöhter Rauschstärke ist in Abb. 8.6 gezeigt. Sie zeigt gute Übereinstimmung mit der im Experiment beobachteten Glättung.

Dass die Rauigkeitsentwicklung für die zwei simulierten Rauschstärken fast gleich ist, lässt sich dadurch erklären, dass die Rauigkeit zu allen simulierten Zeitpunkten durch Strukturen größerer lateraler Ausdehnung dominiert ist. Das bestätigen auch die Oberflächentopographien. Der qualitative Einfluss der Rauschstärke auf die spektralen Leistungsdichten stimmt mit den Erwartungen aus dem linearen Modell gemäß Gleichung (4.11) überein.

Abschließend ist zu sagen, dass mit den Termen, die durch MD-Simulationen genauer untersucht worden sind, die experimentellen Ergebnisse bezüglich der Rauigkeitsentwicklung und der spektralen Leistungsdichten recht gut wiedergegeben werden können, wenngleich noch keine völlige Übereinstimmumg erreicht ist. Daher sollen im Folgenden noch weitere Terme untersucht werden, die als mögliche Beiträge in Frage kommen.

\subsection{Nichtlokale Prozesse}

Modell Da in der Literatur nichtlokale Terme diskutiert werden, soll insbesondere der Einfluss eines Terms der Form aus Gleichung (4.29) untersucht werden. Zunächst wird ein Dämpfungsterm ohne zusätzliche Mittelung zusammen mit einem Rauschterm verwendet, d.h. es wird der Gleichung

$$
\frac{\partial h}{\partial t}=-\kappa h+\xi
$$

entsprechend die Evolution der Ausgangsstruktur berechnet. Dabei werden die zunächst beliebigen Parameter $\kappa=0.1 \mathrm{~s}^{-1}$ und $R=0.0174 \mathrm{~nm}^{4} / \mathrm{s}$ verwendet.

Ergebnisse Die Entwicklung der Rauigkeit zeigt dabei im Anfangsstadium ein exponentielles Abfallen (siehe Abb. 8.7) und stimmt somit nicht mit den experimentellen Ergebnissen überein. In diesem Kapitel ist nicht exakt die Ausgangsstruktur aus Abb. 8.1.a verwendet worden, sondern eine mit anderen Zufallszahlen simulierte, die eine etwas höhere Rauigkeit aufweist. Solche stochastisch bedingten Unterschiede in der Ausgangsstruktur haben aber keinen Einfluss auf die hier diskutierten Ergebnisse zu den untersuchten Termen. In den spektralen Leistungsdichten in Abb. 8.7 ist zu erkennen, dass 
a.)

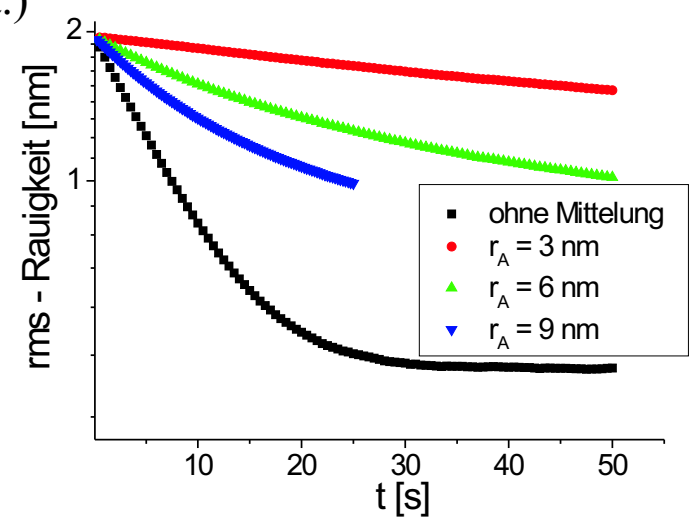

b.)

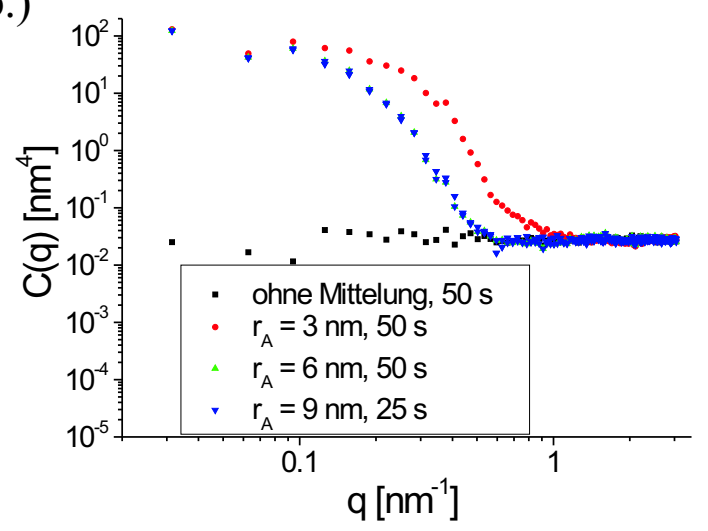

Abbildung 8.7: a.) Entwicklung der rms-Rauigkeit als Funktion der Bestrahlungszeit und b.) spektrale Leistungsdichten jeweils mit verschiedenen lokalen Mittelungen, jeweils nach Kap. 8.3.

$C(q)$ eine Konstante in Abhängigkeit von $q$ ist. Bis auf die stochastische Rauigkeit, die als Grenzfall erreicht wird, ist der Glättungsprozess bereits abgeschlossen.

Modell Um eine Dämpfung, die der Abweichung des Höhenprofils von einer über einer Fläche $A$ gemittelten Höhe proportional ist, zu betrachten, wurde die allgemeine Form der Gleichung (4.29), d.h.

$$
\frac{\partial h}{\partial t}=-\kappa\left(h-\langle h\rangle_{A}\right)+\xi
$$

mit denselben Parametern wie in Gleichung (8.5) simuliert. Für die Fläche $A$, über die gemittelt wird, wird eine Größe angenommen, die ungefähr der Größe des flüssigen Bereichs an der Oberfläche entspricht. Die Mittelung ist im Simulationscode derart implementiert worden, dass für jeden $(\mathrm{x}, \mathrm{y})$ Wert der diskretisierten Substratkoordinaten innerhalb eines Kreises vom Radius $r_{A}$ der Mittelwert der Höhenfunktion in diesem Bereich $\langle h\rangle_{A}$ berechnet wird. In Anlehnung an die in Kap. 7 bestimmte Größe aufgeschmolzener Bereiche und unter Berücksichtigung der etwas höheren Bestrahlungsenergie im Experiment wurden für die lokale Mittelung Radien von $r_{A}=3 \mathrm{~nm}, r_{A}=6 \mathrm{~nm}$ bzw. $r_{A}=9 \mathrm{~nm}$ verwendet.

Ergebnisse Mit diesen Werten ergibt sich ebenso wie zuvor ohne zusätzliche Mittelung im Anfangsstadium ein exponentieller Abfall der Rauigkeit, wobei hier allerdings Abweichungen zu erkennen sind. In den spektralen Leistungsdichten ergibt sich ein Bereich für große $q$-Werte, in dem $C(q)$ konstant ist (siehe hierzu jeweils Abb. 8.7). Ein Unterschied, der sich durch die lokale Mittelung ergibt, ist, dass die Glättung langsamer erfolgt und zwar umso langsamer, je kleiner die Fläche der lokalen Mittelung ist. Je nachdem, wie weit die Struktur bereits geglättet ist, ergibt sich für die spektralen Leistungsdichten ein konstantes Verhalten oberhalb des $q$-Wertes, ab dem die Strukturen entsprechen- 
der Größe bereits geglättet sind. Auch hier deutet sich damit ein Verhalten wie beim Dämpfungsterm ohne lokale Mittelung an.

Diskussion In den Simulationen der Gleichung (8.5) entspricht der exponentielle Abfall der Rauigkeit den theoretischen Erwartungen für die Auswirkung eines linearen Dämpfungsterms aus Kap. 4.7. Ebenso entspricht die Annäherung der spektralen Leistungsdichte an eine Konstante den Erwartungen aus dem linearen Modell gemäß Gleichung (4.11) und steht gleichzeitig im Widerspruch zu den experimentellen Resultaten. Die Einführung der Mittelung gemäß Gleichung (8.6) ändert das Verhalten nicht qualitativ, sondern verlangsamt den Glättungsprozess lediglich.

Mit diesen Ergebnissen ist erwiesen, dass ein linearer Dämpfungsterm (mit oder ohne zusätzliche Mittelung) nicht als dominanter Term die Prozesse beschreibt, die im Experiment für die Strukturentwicklung verantwortlich sind. Weil die spektralen Leistungsdichten im Bereich großer $q$-Werte konstant sind, ist von dem Term auch nicht zu erwarten, dass eine Kombination mit dem Modell aus Kap. 8.2 die dort beobachtete Form des Zerfalls der spektralen Leistungsdichten, also die Steigung in doppeltlogarithmischer Darstellung, im Bereich großer $q$-Werte ändert.

\subsection{Modellierungsansätze zum Volumenfließen}

Modell Zunächst wird eine Gleichung der Form (4.28)

$$
\frac{\partial h}{\partial t}=c_{1}|\nabla h|+\xi
$$

(mit $c_{1}=-\frac{\gamma}{2 \eta}$ ), wie sie in Kap. 4.5.4.2 besprochen worden ist, numerisch gelöst. An dieser Stelle haben sich qualitative Simulationen (also ohne eine quantitative Begründung der Parameter) als ausreichend erwiesen, wie im Folgenden klar werden wird. Die entsprechende Simulation ist mit und ohne den Rauschterm durchgeführt worden. Für den deterministischen Term ist als Koeffizient $c_{1}=-1.0 \mathrm{~s}^{-1}$ verwendet worden. In den Simulationen, in denen ein Rauschterm enthalten ist, gilt $R=0.0174 \mathrm{~nm}^{4} / \mathrm{s}$. Als Ausgangsstruktur dient dieselbe wie in Kap. 8.3. Um zu gewährleisten, dass die entsprechende Strukturentwicklung durch die Form der Gleichung und nicht durch numerische Instabilitäten bedingt ist, ist die Gleichung zusätzlich mit einer um den Faktor 10 kleineren Zeitschrittweite gelöst worden. Im Simulationscode ist der $|\nabla h|$-Term durch einen im Ort symmetrischen Differenzenquotienten im Rahmen des Euler-Verfahrens (siehe Kap. 4.8) analog zur Diskretisierung von Ableitungen erster Ordnung im Ort in [May00; Vau02] implementiert worden.

Ergebnisse Zunächst zeigt sich für die Simulation von Gleichung (8.7), dass die Entwicklung der Rauigkeit eine deutliche Aufrauung zeigt. Um auszuschließen, dass der Rauschprozess der Grund für diese Aufrauung ist, wird zusätzlich der deterministische 


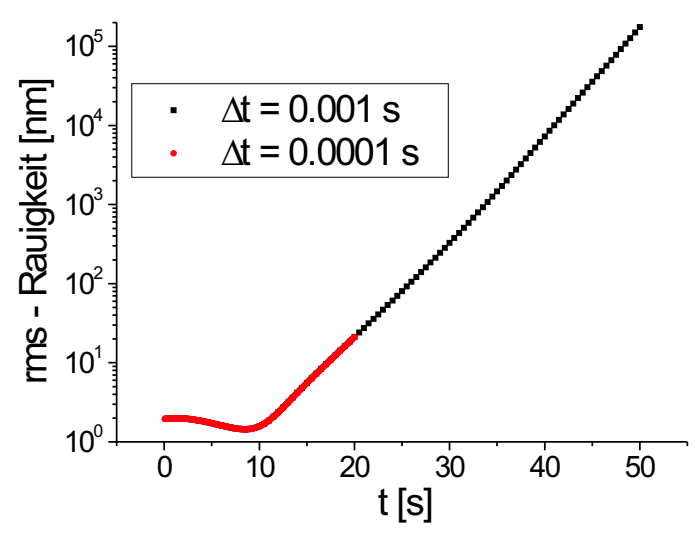

Abbildung 8.8: Entwicklung der rms-Rauigkeit als Funktion der Bestrahlungszeit für einen Glättungsterm der Form $|\nabla h|$. Bis $t=20 \mathrm{~s}$ liegen schwarze und rote Symbole übereinander.

Fall gelöst. Die Rauigkeitsentwicklung im deterministischen Fall ist in Abb. 8.8 dargestellt. Für $\Delta t=0.001 \mathrm{~s}$ zeigt sie keine erkennbare Abweichung von der Rauigkeitsentwicklung, die sich bei Berücksichtigung des Rauschterms ergibt. Weiterhin zeigt die Simulation mit der Zeitschrittweite $\Delta t=0.0001 \mathrm{~s}$ keinen erkennbaren Unterschied zur größeren Zeitschrittweite, so dass beide Werte von $\Delta t$ eine stabile Lösung liefern. Daraus ergibt sich, dass der zu $|\nabla h|$ proportionale Term die Ursache der Aufrauung ist. In der zugehörigen Rauigkeitsentwicklung in Abb. 8.8 zeigt sich, dass die Rauigkeit zwar zunächst etwas abnimmt, aber stets bei Werten oberhalb von $1 \mathrm{~nm}$ bleibt. Daran anschließend wächst sie exponentiell an und divergiert. Dieses Verhalten kann nicht dem Verhalten entsprechen, was zu erwarten ist, wenn viskoses Volumenfließen der einzige Prozess ist, denn der ist glättend. Abgesehen von der Rauigkeitsentwicklung zeigen für die hier simulierte Gleichung die Oberflächentopographien auch in dem Bereich, in dem die Rauigkeit ein Minimum erreicht hat, bereits qualitativ ein deutlich anderes Verhalten, als es für einen glättenden Fließprozess zu erwarten ist. Ein Vergleich der Oberfläche zum Zeitpunkt des Rauigkeitsminimums mit der Ausgangsstruktur in Abb. 8.9 zeigt, dass die Hügelspitzen übrig bleiben, während an den Hügelflanken die Werte der Höhenfunktion stark abnehmen. Dadurch ergeben sich große Steigungen, die den Effekt weiter verstärken. Die Simulation bestätigt, dass die Gleichung die Teilchenzahl nicht erhält, denn die über die gesamte Struktur gemittelte Höhe nimmt als Funktion der Zeit ab, wie es in Kap. 4.5.4.2 bereits begründet worden ist.

Diskussion Die Ergebnisse deuten darauf hin, dass die Gleichung (8.7) unrealistisch ist, weil die Höhe an den steilsten Stellen der Oberfläche am stärksten abnimmt. Entspricht die Höhe in einem steilen Bereich aber gerade der mittleren Höhe des aufgeschmolzenen Bereichs, so ist an dieser Stelle für einen Fließprozess keine Höhenänderung zu erwarten (siehe Abb. 8.10), aber die Gleichung (8.7) bewirkt gerade dort eine star- 
a.)

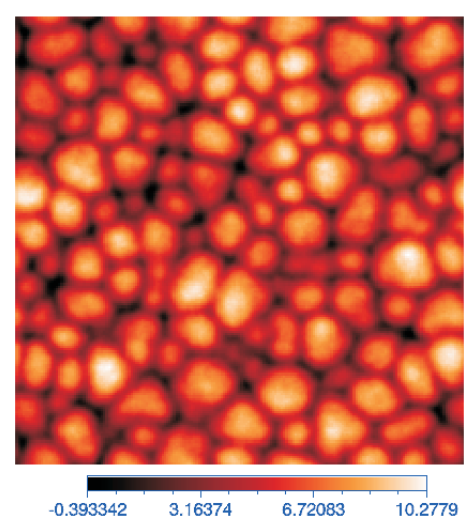

b.)

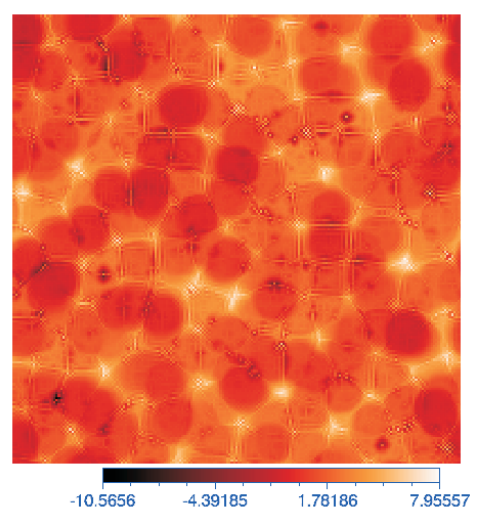

Abbildung 8.9: Oberflächentopographien der Ausgangsstruktur sowie im Zeitbereich des Rauigkeitsminimums für die Simulation eines Glättungsterms der Form $|\nabla h|$ (Bildgröße: $L=200$ nm, Einheit der Höhenskala: nm).

ke Höhenänderung. Dass die mittlere Höhe mit der Zeit abnimmt, entspricht der in Kap. 4.5.4.2 begründeten Eigenschaft der Gleichung, dass stets $\partial h / \partial t \leq 0$ gilt. Damit ist gezeigt, dass Gleichung (8.7) keinen Fließprozess beschreibt, wie es bereits in Kap. 4.5.4.2 anhand der Form der Gleichung begründet worden ist.

Modell Da der bisher besprochene Term offensichtlich kein viskoses Volumenfließen beschreiben kann, wurden weitere Terme untersucht, die für eine angemessene Beschreibung des Volumenfließens in Frage kommen. Ein solcher Term sollte in linearisierter Form durch Fouriertransformation die Form von Gleichung (4.27) erfüllen. Dafür ist es notwendig, dass im Ortsraum eine räumliche Ableitung erster Ordnung enthalten ist. Da es Hinweise gibt, dass viskoses Volumenfließen nicht durch einen lokal bestimmten Term beschrieben werden kann [UHC01], müsste auch ein nichtlokaler Beitrag in den Term, der viskoses Volumenfließen korrekt beschreibt, eingehen. Außerdem sollte durch viskoses Volumenfließen die Teilchenzahl erhalten bleiben. Ein Term, der sowohl den nichtlokalen Charakter als auch eine räumliche Ableitung erster Ordnung beinhaltet, ist durch die folgende Gleichung gegeben:

$$
\frac{\partial h}{\partial t}=\tilde{c}_{1}\left(h-\langle h\rangle_{A}\right) \cdot|\nabla h|+\xi, \quad \tilde{c}_{1}<0 .
$$

Diese Gleichung ist allerdings nichtlinear und liefert durch Fouriertransformation nicht die Gleichung (4.27). Gegenüber Gleichung (8.7) hat sie mehrere Vorteile. Bereiche, die bereits mit ihrer Höhe dem Mittelwert des aufgeschmolzenen Bereichs entsprechen, werden nicht mehr bezüglich ihrer Höhe geändert. Für eine um die mittlere Höhe symmetrische Höhenfunktion erhält sie außerdem die Teilchenzahl, weil die Höhenänderungsrate zu gleichen Anteilen positiv und negativ ist. Die Teilchenzahlerhaltung gilt für Gleichung (8.8) sogar für eine beliebige Höhenfunktion, wie sich durch Berechnung der Eigenschaft $\int_{\text {Oberfläche }} \frac{\partial h}{\partial t} d x d y=0$ ergibt (siehe Anhang 11.4). 


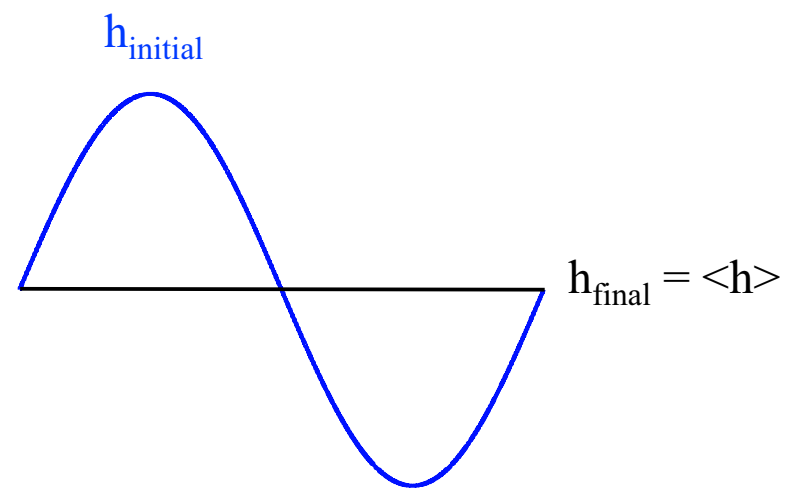

Abbildung 8.10: Erwarteter Einfluss eines Fließprozesses auf die Entwicklung eines sinusförmigen Höhenprofils.

Ergebnisse Eine Gleichung entsprechend (8.8) führt mit den Parametern $\tilde{c}_{1}=-1.01 / \mathrm{s}$, dem Radius der Mittelung $r_{A}=3 \mathrm{~nm}$ und der Rauschstärke $R=0.02 \mathrm{~nm}^{4} / \mathrm{s}$ für die Ausgangsstruktur aus Abb. 8.1.a zu einer Glättung. Um einen stärkeren Glättungseffekt $\mathrm{zu}$ erreichen, ist Gleichung (8.8) auch mit dem Parameter $\tilde{c}_{1}=-10.01 / \mathrm{s}$ simuliert worden. Die Entwicklung der Rauigkeit für beide Parameter sowie die Entwicklung der spektralen Leistungsdichten für den betragsmäßig größeren Wert von $\tilde{c}_{1}$ sind in Abb. 8.11 dargestellt. Die Entwicklung der Rauigkeit nähert sich für größere Zeiten bzw. für einen betragsmäßig größeren Wert von $\tilde{c}_{1}$ einem Potenzgesetz, während zu Beginn ein schwächerer Abfall zu verzeichnen ist. Mit Zunahme der Zeit bildet sich in den spektralen Leistungsdichten im Bereich $0.4 \mathrm{~nm}^{-1}<q<2 \mathrm{~nm}^{-1}$ immer deutlicher ein Bereich heraus, in dem sich der Verlauf dem Verhalten $C(q) \propto q^{-1}$ annähert. Die Topographien in Abb. 8.12 zeigen eine ähnliche Strukturentwicklung wie die zuvor gezeigten Glättungsterme: Die Strukturen werden lateral größer und verlieren gleichzeitig an Höhe in der Richtung der Substratnormalen. Die Simulation zeigt außerdem, dass die über die gesamte Struktur gemittelte Höhe bei Anwendung dieser Gleichung konstant bleibt, was der oben erläuterten Teilchenzahlerhaltung entspricht.

Diskussion Der visuelle Eindruck der Topographien zeigt eine Strukturänderung, wie sie für ein Zerfließen der ursprünglichen Strukturen zu erwarten ist. Zusammen mit dem für die spektralen Leistungsdichten in einem begrenzten Wellenlängenbereich beobachteten Verhalten gemäß $C(q) \propto q^{-1}$ deutet das darauf hin, dass der hier simulierte Glättungsterm Volumenfließen näherungsweise wiedergeben kann. Dagegen spricht aber, dass die Rauigkeit gemäß einem Potenzgesetz abfällt, wobei der Exponent dem Betrage nach deutlich kleiner als 1 ist, wohingegen nach den Überlegungen in Kap. 4.7 als Exponent $-1 \mathrm{zu}$ erwarten ist.

Dass für große $q$-Werte die spektrale Leistungsdichte für das Modell des Volumenfließens größer als bei der gewachsenen Schicht ist, lässt sich durch die Form der Glei- 
a.)

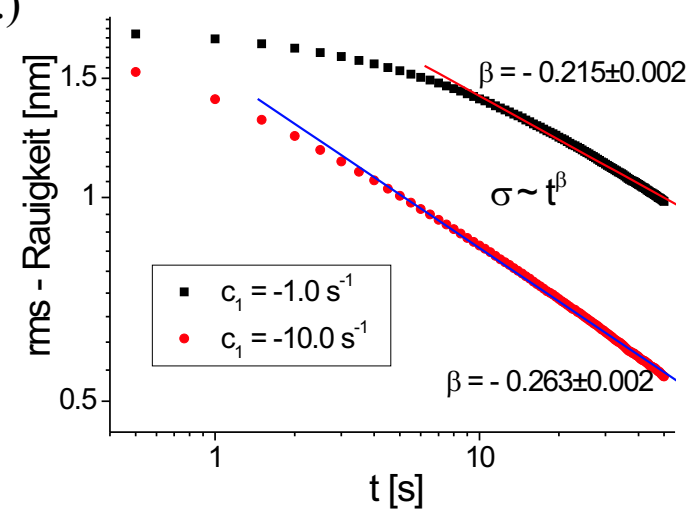

b.)

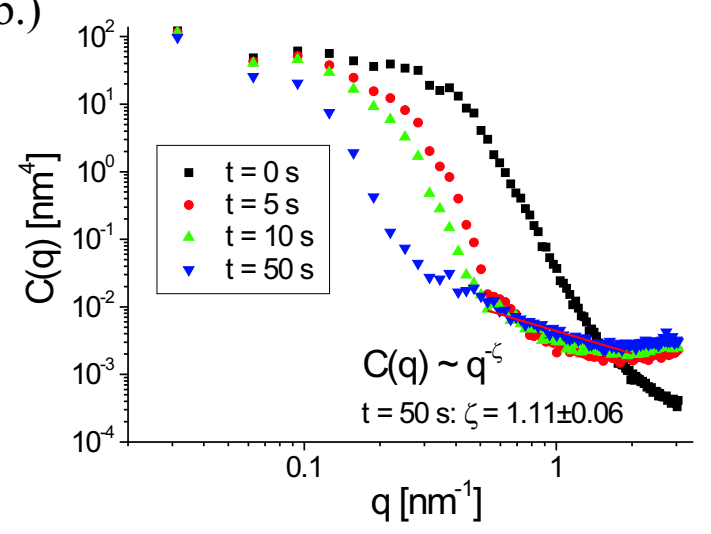

Abbildung 8.11: a.) Entwicklung der rms-Rauigkeit als Funktion der Bestrahlungszeit und b.) spektrale Leistungsdichten für $\tilde{c}_{1}=-10 \mathrm{~s}^{-1}$, jeweils nach Gleichung (8.8).

chung (4.12) erklären: Wenn nur ein Term erster Ordnung vorhanden ist, wird der Ausdruck im Nenner betragsmäßig kleiner. Auf kleinen Skalen im Ortsraum glättet ein Term erster Ordnung weniger effektiv als ein Term höherer Ordnung.

\subsection{Oberflächenfließen, ballistisches Glätten und Volumenfließen}

Modell In diesem Kapitel wird überprüft, ob sich das Modell aus Kap. 8.2 durch Kombination mit dem Glättungsterm aus Gleichung (8.8) hinsichtlich der Übereinstimmung mit den experimentellen Ergebnissen weiter verbessern lässt. Dafür wird eine Gleichung der Form

$$
\frac{\partial h}{\partial t}=c_{2} \nabla^{2} h+c_{4} \nabla^{4} h+\tilde{c}_{1}\left(h-\langle h\rangle_{A}\right) \cdot|\nabla h|+\xi
$$

auf die Ausgangsstruktur angewendet. Für die in Kap. 8.2 berücksichtigten Terme werden hier die dort quantitativ begründeten Werte für die zugehörigen Parameter verwendet, d.h. $c_{2}=8.06 \cdot 10^{-3} \mathrm{~nm}^{2} / \mathrm{s}, c_{4}=-0.810 \mathrm{~nm}^{4} / \mathrm{s}$ und $R=5.38 \cdot 10^{-5} \mathrm{~nm}^{4} / \mathrm{s}$. Für den zusätzlichen Term werden dieselben Parameter wie in Kap. 8.4 verwendet, d.h. $\tilde{c}_{1}=-1.0 \mathrm{~s}^{-1}$ und $r_{A}=3 \mathrm{~nm}$. Dabei ist zu berücksichtigen, dass es für den Wert von $\tilde{c}_{1}$ keine quantitative Rechtfertigung gibt.

Ergebnisse Die Entwicklung der Rauigkeit sowie der Verlauf der spektralen Leistungsdichten, die sich aus der numerischen Simulation des Modells ergeben, sind in Abb. 8.13 dargestellt. Die Entwicklung der Rauigkeit lässt sich im Bereich größerer Zeit durch ein Potenzgesetz annähern. Im Vergleich zu den in den vorhergegangenen Kapiteln durchgeführten Simulationen von Termen, aus denen die Gleichung in diesem Kapitel zusammengesetzt ist, liegt der Wert des Exponenten $\beta$ weiter vom experimentellen Wert 
a.)

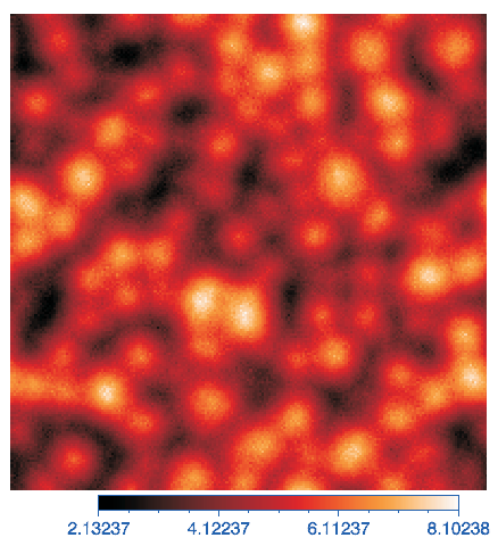

b.)

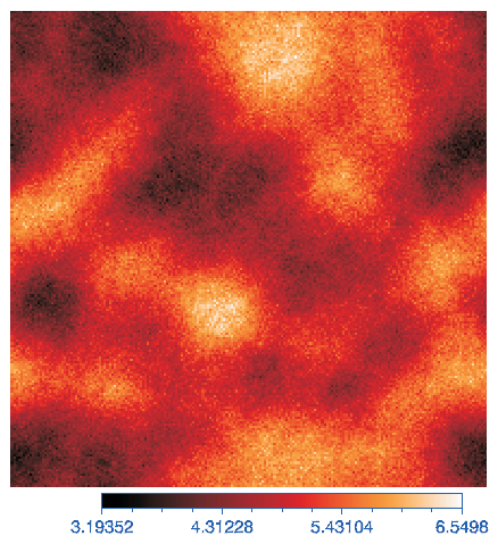

Abbildung 8.12: Topographie der gemäß Gleichung (8.8) simulierten Bestrahlung mit $\tilde{c}_{1}=-10 \mathrm{~s}^{-1}$ für Zeiten von a.) $5 \mathrm{~s}$ und b.) $50 \mathrm{~s}$ (Bildgröße: $L=200 \mathrm{~nm}$, Einheit der Höhenskala: nm).

entfernt als es die Ergebnisse aus Kap. 8.2 zeigen. Die spektralen Leistungsdichten sind in ihrem Verlauf dagegen sehr ähnlich zu denen aus Kap. 8.2. Der Exponent $\zeta$ ist durch Hinzunahme des Terms, der in Verbindung zum Prozess des Volumenfließens steht, nur wenig verkleinert worden. Im Bereich großer $q$-Werte liegen die Werte der spektralen Leistungsdichte unter denen, die sich in Kap. 8.2 mit derselben Rauschstärke und denselben Werten für $c_{2}$ und $c_{4}$ ergeben haben. Dies zeigt ein Vergleich der Abbildungen 8.5.b und 8.13.b.

Diskussion Durch die Hinzunahme des nichtlinearen Terms nähern sich die Ergebnisse der Simulationen denen der Experimente nicht eindeutig an. Die Rauigkeitsentwicklung in Kap. 8.2 ist näher am experimentellen Resultat als das in diesem Abschnitt simulierte Modell. Der in diesem Abschnitt bestimmte Wert von $\beta$ ist allerdings nicht direkt mit dem experimentellen vergleichbar, weil die Rauigkeit hier noch nicht so weit abgesunken ist wie im Experiment. Die im nichtlinearen Term enthaltene lokale Mittelung vergrößert die Berechnungszeit für einen Integrationsschritt, so dass die Simulationen nur bis $t=500 \mathrm{~s}$ durchgeführt worden sind.

Der Exponent $\zeta$ wird durch Hinzunahme des nichtlinearen Terms gegenüber den Ergebnissen au Kap. 8.2 kaum verändert. Dies entspricht der Dominanz der Terme mit Ableitungen höherer Ordnung, die gemäß Gleichung 4.12 zumindest in linearer Näherung gültig ist. Außerdem führt das Hinzufügen des nichtlinearen Terms dazu, dass im Bereich großer $q$-Werte die Amplitude der spektralen Leistungsdichte abnimmt. So würde man es für einen zusätzlichen linearen Glättungsterm im Rahmen des linearen Modells aus Kap. 4.3 erwarten, weil in Gleichung (4.11) der Nenner des zweiten Summanden durch einen zusätzlichen glättenden Term vergrößert wird. Zumindest qualitativ hat der nichtlineare Term denselben Effekt, wenngleich die Erklärung nicht direkt anwendbar ist. 
a.)

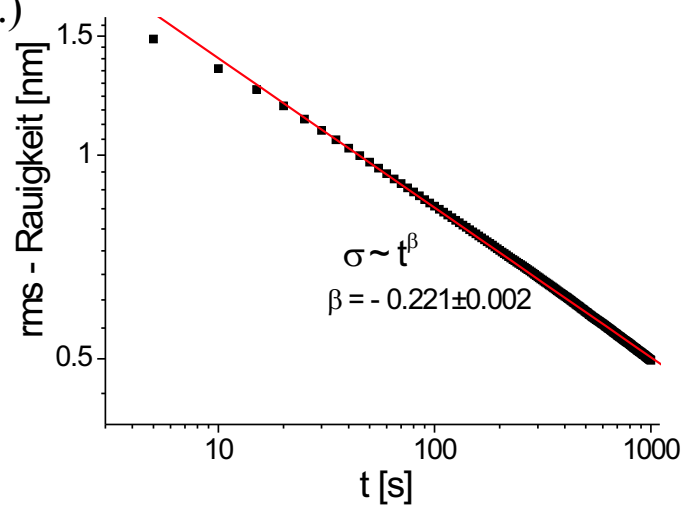

b.)

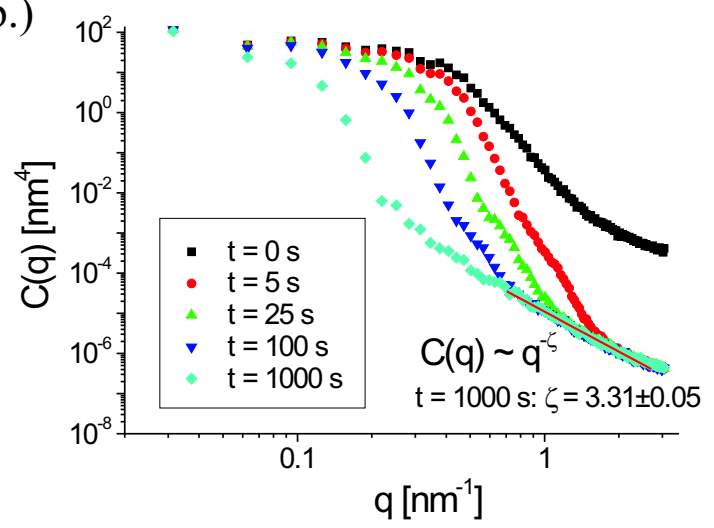

Abbildung 8.13: a.) Entwicklung der rms-Rauigkeit als Funktion der Bestrahlungszeit und b.) spektrale Leistungsdichten für die Simulation des Modells aus Gleichung (8.9).

Eine weitere Untersuchung des Volumenfließens, insbesondere auch in quantitativer Weise, könnte hier weiteren Fortschritt bringen.

\subsection{Diskussion}

In diesem Kapitel sind verschiedene Modelle zur Beschreibung des Effekts der Ionenbestrahlung von strukturierten Oberflächen numerisch gelöst worden. Die Details der untersuchten Modelle sind bereits in den einzelnen Abschnitten diskutiert worden, so dass an dieser Stelle die wesentlichen Ergebnisse vor dem Hintergrund der bestehenden Literatur zusammengefasst und eingeordnet werden.

Unabhängig von den MD-Simulationen aus Kap. 7 dieser Arbeit ergeben die Simulationen der Kontinuumsmodelle in Kap. 8.1 und Kap. 8.2, dass ein glättender $\nabla^{2} h$ Term in Kombination mit einem glättenden $\nabla^{4} h$-Term die experimentellen Ergebnisse deutlich besser wiedergeben kann als ein aufrauender $\nabla^{2} h$-Term mit einem glättenden $\nabla^{4} h$-Term. Damit ist gezeigt, dass die Theorie nach Bradley und Harper [BH88] die experimentell untersuchten Effekte nicht erklären kann. Dagegen kommt das Modell in Kap. 8.2, das bisher in dieser Form in der Literatur noch nicht untersucht worden ist, den experimentellen Ergebnissen deutlich näher. Das Modell aus Kap. 8.2 liefert bereits weitreichende Übereinstimmung mit dem Experiment und ist somit für Vorhersagen der durch Bestrahlungsprozesse getriebenen Strukturentwicklung in einem großen Intervall von Bestrahlungszeiten bzw. Fluenzen anwendbar.

Als Grund dafür, dass der Exponent $\zeta$ in den untersuchten Simulationen zur Bestrahlung tendenziell größer als im Experiment ist, lässt sich generell anführen, dass die Wachstumsgleichung (4.13) aufgrund der enthaltenen Nichtlinearitäten für Schichtdicken im Bereich von $480 \mathrm{~nm}$ Abweichungen von dem durch Gleichung (4.10) beschriebenen Verhalten aufweist. In einigen Bereichen des $q$-Werts ist der Abfall der spektralen 
Leistungsdichte steiler als $q^{-4}$. Falls solche Abweichungen erst nach sehr langen Zeiten während der Simulation der Bestrahlungsprozesse verschwinden, könnten sie die für $\zeta$ bestimmten Werte beeinflussen und zu einem zu großen Wert von $\zeta$ führen. Allerdings lässt sich der Einfluss von nichtlinearen Termen auf die spektralen Leistungsdichten im Rahmen des linearisierten Modells aus Kap. 4.3 nicht klären.

Die Abweichungen bezüglich des Exponenten $\zeta$ lassen sich bereits im linearen Modell noch überzeugender erklären. In den simulierten Modellen in Kap. 8.2 ist Oberflächenfließen mit ballistischem Glätten kombiniert worden, so dass $\zeta$ zwischen 2 und 4 liegt. Für die betrachteten Bestrahlungsenergien und Strukturgrößen ist zwar Oberflächenfließen der dominierende Fließprozess, aber der alleinige Fließprozess ist es nur im Grenzfall $a_{z} / \lambda \ll 1$ [Orc62]. Im realen Fall handelt es sich immer um eine Kombination von Volumenfließen und Oberflächenfließen. Die Analyse des Modells von Orchard [Orc62] führt dazu, dass sich in der spektralen Leistungsdichte bei einer Kombination der beiden Fließprozesse ein Exponent $\zeta$ zwischen den Werten 1 und 4 ergibt. Dabei gilt $\zeta=4$ falls $a_{z} / \lambda \ll 1$ und $\zeta=1$ falls $a_{z} / \lambda \gg 1$. Der durch Fließprozesse erzeugte Wert von $\zeta$ sollte im Experiment also nicht exakt 4 sondern etwas kleiner sein. In Kombination mit ballistischem Glätten sollte dieses Argument bestehen bleiben, so dass der sich insgesamt ergebende Exponent $\zeta$ kleiner ist, als es eine Kombination aus reinem Oberflächenfließen und ballistischem Glätten erwarten lässt. Damit lässt sich erklären, dass experimentell ein kleinerer Wert für $\zeta$ gefunden wird als in der Simulation eines Modells, in dem Oberflächenfließen der einzige Fließprozess ist.

Bei allen in diesem Kapitel simulierten Modellen ergibt sich eine Glättung bei gleichzeitiger Vergrößerung der Strukturgröße. Wenn alle deterministischen Terme der Gleichung glättenden Charakter haben, ist das im Einklang mit dem linearen Modell aus Kap. 4.3: Die durch das Wachstum hervorgerufenen Korrugationen klingen mit der Zeit ab und zwar umso schneller je größer der $q$-Wert ist. Dementsprechend verschwinden kleine Strukturen schneller als große und damit steigt die mittlere Strukturgröße mit der Zeit an. Im Modell von Bradley und Harper [BH88] führt das Zusammenspiel von glättendem $\nabla^{4} h$-Term und aufrauendem $\nabla^{2} h$-Term dagegen zu einer Instabilität wie es sie auch beim Schichtwachstum gibt $\left[\mathrm{RML}^{+} 00\right]$. Demnach gibt es eine ausgezeichnete Wellenlänge, die die Struktur dominiert. Der Wert dieser Wellenlänge ergibt sich aus dem Verhältnis der Koeffizienten und bei entsprechender Wahl kann auch in diesem Modell die Strukturgröße als Funktion der Zeit wachsen, wie es auch in Kap. 8.1 beobachtet wird.

Da die Modelle mit linearen Dämpfungstermen aus Kap. 8.3 wegen der dort gezeigten und diskutierten Ergebnisse nicht wesentlich zu der im Experiment beobachteten Entwicklung beitragen, können diese Terme hier außer Acht gelassen werden. Die Ansätze zum Volumenfließen sind bisher noch nicht vollständig geklärt und physikalisch begründet. Somit muss das Modell aus Kap. 8.2, das zum einen physikalisch begründet ist und zum anderen in weiten Teilen ähnliche Ergebnisse wie die Experimente liefert, zum jetzigen Zeitpunkt als das beste Modell angesehen werden. Außerdem ist für die in dieser Arbeit experimentell verwendeten Bestrahlungsenergien in Verbindung mit den bestrahlten Strukturen das Oberflächenfließen gegenüber dem Volumenfließen dominant. Die Modellierung des Volumenfließens im Ortsraum hat an dieser Stelle demnach nur 
einen untergeordneten Stellenwert.

Für die Zukunft ist aber die Entwicklung eines physikalisch vollständig begründeten Modells für den Prozess des Volumenfließens im Ortsraum wünschenswert. Damit wäre dann auch die Modellierung der Strukturentwicklung bei höheren Bestrahlungsenergien, für die Volumenfließen gegenüber Oberflächenfließen an Bedeutung gewinnt, im Ortsraum möglich.

Eine in den Experimenten beobachtete schwache Aufrauung bei der kleinsten Fluenz (vgl. Diskussion 5.5.1 wird durch keines der untersuchten Kontinuumsmodelle wiedergegeben. Für den Fall, dass sämtliche deterministischen Terme glättend sind, entspricht das der Erwartung. Bei dem Modell in Kap. 8.1 ist im Vergleich zur Wachstumsgleichung das Gewicht des glättenden Terms erhöht worden, so dass die Korrelationslänge zu- und die Rauigkeit abnimmt. Von Streng [Str04; SSM06] wurde eine kurzfristige Aufrauung und eine Verkleinerung der Korrelationslänge beobachtet, wenn im Kontinuumsmodell das Gewicht des glättenden $\nabla^{4} h$-Terms verringert wird. Da eine solche Änderung im Kontinuumsmodell die Bestrahlung, die eine Vergrößerung der Korrelationslänge bewirkt, nicht beschreiben kann, legen die Kontinuumsmodelle nahe, dass es sich bei der Erhöhung der Rauigkeit für die niedrigste Fluenz im Experiment um eine statistisch bedingte Abweichung handelt.

Des Weiteren ist zu erwähnen, dass das Modell in Kap. 8.2 lediglich lineare Terme enthält. Für den Prozess des Oberflächenfließens werden aber auch nichtlineare Terme diskutiert [Orc62; SL88], die im Grenzfall kleiner Höhenmodulationen in den linearen Term, der hier zur Beschreibung des Oberflächenfließens verwendet wird, übergehen. Die Simulationen haben gezeigt, dass mit den in linearer Näherung verwendeten Termen die wesentlichen Tendenzen, die im Experiment beobachtet werden, wiedergegeben werden können. Außerdem sollten die linearen Terme insbesondere für den stationären Grenzfall einer glatten Schicht, der sich für große Zeiten ergibt, immer die dominante Rolle spielen, weil die Bedeutung der nichtlinearen Terme umso mehr abnimmt, je kleiner die Höhenmodulationen werden. Weitere nichtlineare Terme werden als Erweiterung der Theorie von Bradley und Harper [BH88] beispielsweise in Form einer KuramotoSivashinsky-Gleichung diskutiert [MCB02]. Die nichtlinearen Erweiterungen werden dort als Erweiterungen für die Beschreibung der Sputtererosion diskutiert. Weil gezeigt werden konnte, dass in dieser Arbeit der Prozess der Sputtererosion nur eine untergeordnete Rolle spielt, gilt dies auch für die zugehörigen nichtlinearen Erweiterungen. Auch für diese Nichtlinearitäten gilt das Argument, dass sie in Annäherung einer glatten Schicht im Vergleich zu den linearen Termen an Bedeutung verlieren.

Für die Aufstellung von Evolutionsgleichungen des Typs aus Gleichung (4.2) lassen sich mögliche Terme durch Symmetrieforderungen erzeugen [BS95]. Die Erfüllung der notwendigen Symmetriebedingungen durch die untersuchten Terme wird im Folgenden kurz erläutert. Alle Terme sind invariant bezüglich Translation in der Zeit und Substratebene. Ebenfalls sind alle Terme invariant bezüglich Rotation in der Substratebene. Diese Symmetrie ist zwar für $\nabla h$ nicht erfüllt aber für $|\nabla h|$. Die Symmetrie bezüglich Translation in z-Richtung gilt für alle Terme außer dem in Gleichung (8.5). Das Subtrahieren einer gemittelten Höhe wie in Gleichung (8.6) führt dazu, dass diese Symmetrie wieder erfüllt ist. Somit erfüllen insgesamt alle untersuchten Terme außer dem in Glei- 
chung (8.5), von dem gezeigt worden ist, dass er für die experimentell beobachteten Effekte keine wesentliche Bedeutung hat, die geforderten Symmetrien. 


\section{Diskussion}

Ein zentrales experimentelles Ergebnis dieser Arbeit ist, dass sich strukturierte Oberflächen eines metallischen Glases durch Ionenbeschuss im Energiebereich weniger keV glätten lassen. Neben diesem phänomenologischen Ergebnis ist die Beantwortung der Frage nach den zu dieser Glättung beitragenden Mechanismen die wesentliche Aufgabe, die in dieser Arbeit bearbeitet werden sollte.

Durch die Analyse der experimentellen Daten im Rahmen der Theorie von Kontinuumsmodellen lassen sich erste Aussagen zur Beantwortung dieser Frage treffen. Eine genauere Abgrenzung der Mechanismen, die zur beobachteten Strukturentwicklung beitragen, ist mit Hilfe atomistischer Simulationen möglich. Mechanismen, die sich nach diesen Untersuchungen als relevant herausstellen, können wiederum in Kontinuumsmodelle eingebaut werden, deren numerische Lösungen sich mit den experimentellen Ergebnissen vergleichen lassen. Innerhalb dieses Konzepts werden im Folgenden die Ergebnisse der einzelnen Kapitel in Zusammenhang gebracht und die wesentlichen Ergebnisse diskutiert. Anschließend werden sie mit aus der Literatur bekannten Untersuchungen zur Bestrahlung anderer Materialysteme verglichen.

\subsection{Ergebnisse der angewendeten Untersuchungsmethoden}

Die experimentellen Ergebnisse zeigen, dass insgesamt Glättungseffekte deutlich größere Beiträge als aufrauende Prozesse haben. Die Analyse im Rahmen eines linearen Evolutionsmodells gemäß Kap. 4.3 und Kap. 4.7 zeigen, dass nicht nur Prozesse der Ordnung $q^{4}$ für die Strukturentwicklung verantwortlich sein können. Es müssen auch Prozesse kleinerer Ordnung relevant sein. Wenn verschiedene physikalische Prozesse mit gleicher Ordnung in das lineare Modell eingehen, kann die Analyse der STM-Daten in diesem Modell prinzipiell nicht beantworten, welcher von den Prozessen gleicher Ordnung dominierend ist. Deshalb sind zur genaueren Klärung der relevanten Prozesse weitere Untersuchungen notwendig. Als solche können die hier durchgeführten MD-Simulationen dienen. Sie zeigen insbesondere durch den Vergleich zu thermisch aktivierten Prozessen, dass durch den Einschuss von Atomen auf die Oberfläche Fließprozesse induziert werden, während bei thermischer Aktivierung ein diffusiver Transportmechanismus vorherrscht. Neben den Fließprozessen konnte in den MD-Simulationen auch die Relevanz ballistischer Effekte gezeigt werden. Das in den MD-Simulationen gefundene Zusammenspiel von Fließprozessen und ballistischer Glättung passt auch zur Analyse der experimentellen Ergebnisse. In Übereinstimmung damit gibt die Kontinuumsmodellierung einer Kombination beider Prozesse die experimentellen Ergebnisse weitgehend wieder. 


\section{Diskussion}

Somit konnten aus einer Kombination der in dieser Arbeit verwendeten Methoden die relevanten Strukturbildungsprozesse während der Ionenbestrahlung amorpher Oberflächen identifiziert werden. Die Berücksichtigung verschiedener Skalen in den verschiedenen Methoden führt zu einer Verbindung dieser Skalen. Es konnte gezeigt werden, wie atomistische Vorgänge und Strukturentwicklung im Größenbereich $10 \mathrm{~nm}$ bis $100 \mathrm{~nm}$ miteinander verknüpft sind. Insbesondere hat die Verknüpfung der MD-Simulationen mit Kontinuumsmodellen dazu geführt, dass für die Koeffizienten in den Kontinuumsmodellen quantitative Werte bestimmt werden können. Die Verknüpfung von atomistischer und mesoskopischer Skala bildet allgemein den Schlüssel zum Verständnis der Entwicklung von Nanostrukturen, da das Verhalten der Atome immer der Ursprung der Strukturentwicklung ist.

In der Literatur zur Untersuchung von Strukturentwicklung durch Ionenbeschuss ist bisher oftmals dem Modell von Bradley und Harper [BH88] folgend von einer Oberflächendiffusion als wesentlichem Glättungsprozess ausgegangen worden. Hier konnte gezeigt werden, dass es sich vielmehr um ein viskoses Oberflächenfließen und nicht um einen diffusiven Prozess handelt. Fließprozesse waren bisher für deutlich höhere Bestrahlungsenergien bekannt, bei denen ein Bereich, der im Verhältnis zur Oberflächenstruktur groß ist, aufschmilzt. Es handelt sich dabei um den Bereich von Bestrahlungsenergien von etwa einem MeV [MA01] bis zu mehreren hundert MeV [KHS86]. Für diese hohen Energien entspricht der Fließprozess genauer gesagt einem Volumenfließen (vgl. Kap. 4.5.4). Im Bereich weniger keV Bestrahlungsenergie waren die Prozesse bisher nicht geklärt [UHC01; MCH94; FSB00; $\mathrm{EAC}^{+} 99$ ]. Die Idee, dass es sich bei dem Glättungsmechanismus, der durch einen $\nabla^{4} h$-Term dargestellt wird, eher um einen Fließ- als um einen Diffusionsprozess handelt, ist zwar schon zuvor diskutiert worden [UHC01], aber bisher fehlte ein quantitativer Beweis, dass dies für Bestrahlungen im Energiebereich weniger keV zutrifft. Dieser konnte hier erbracht werden. Insgesamt werden in der vorhandenen Literatur bisher Kontinuumsmodelle, in denen verschiedene Prozesse berücksichtigt werden, in der Regel qualitativ betrachtet, oder es werden die Koeffizienten durch physikalische Größen beschrieben, deren Werte unbekannt sind. Der Schritt zur Bestimmung der Werte durch realistische MD-Simulationen ist hier durchgeführt worden.

Der Prozess des ballistischen Glättens ist in quantitativer Weise bisher nur von Moseler et al. untersucht worden $\left[\mathrm{MGC}^{+} 05\right]$. Hier konnte dieser Effekt auch für metallische Gläser gefunden und in quantitativ begründeter Weise in Kombination mit anderen Prozessen in Kontinuumsmodellen betrachtet werden.

Die zugehörigen Simulationen des Kontinuumsmodells, in dem viskoses Oberflächenfließen, ballistisches Glätten sowie ein Rauschprozess kombiniert werden, können die experimentellen Resultate im Wesentlichen wiedergeben. Es fällt bei der Rauigkeitsentwicklung aber auf, dass im Experiment die Rauigkeit in größerer Übereinstimmung mit einem Potenzgesetz ist, als es beim Kontinuumsmodell der Fall ist. Dieser Eindruck hat zum Teil seinen Ursprung darin, dass in der Simulation kleinere Zeiten dargestellt werden können, als im Experiment untersucht worden sind. Um dies zu verdeutlichen, sind in Abb. 9.1 die experimentellen Daten zusammen mit den Simulationsdaten dargestellt. Hier sind also experimentelle Ergebnisse zusammen mit einer Kontinuumsgleichung, deren Koeffizienten aus MD-Simulationen bestimmt sind, zusammen aufgetragen und 
a.)

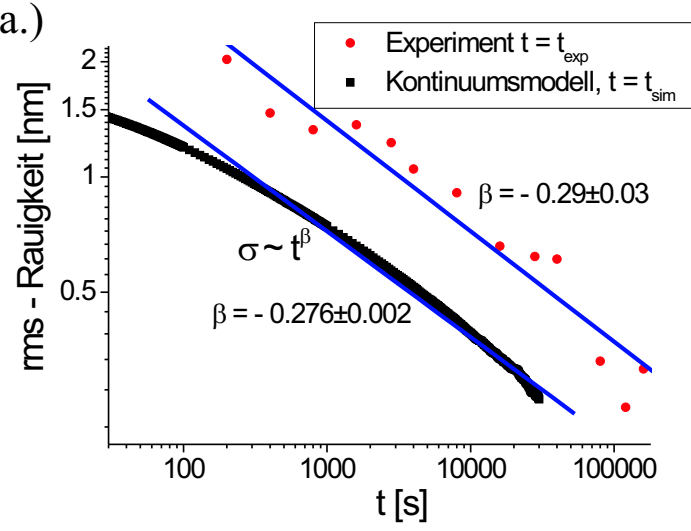

b.)

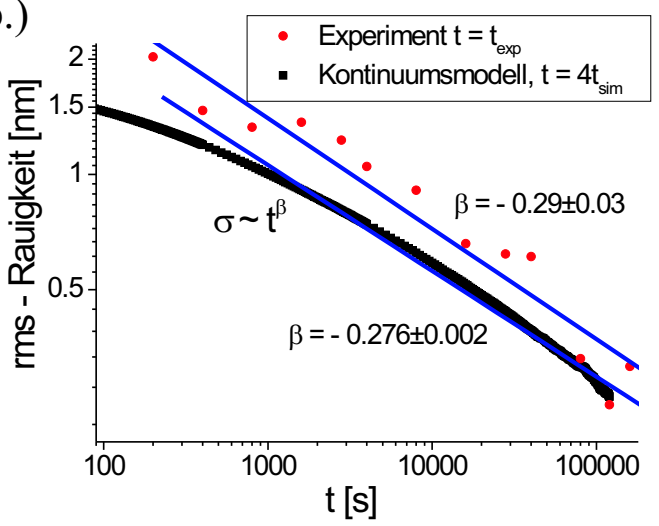

Abbildung 9.1: Vergleich der Rauigkeitsentwicklung im Experiment und im Kontinuumsmodell gemäß Kap. 8.2 mit $R=5.38 \cdot 10^{-5} \mathrm{~nm}^{4} / \mathrm{s}$. Für Daten des Kontinuumsmodells wird in a.) der Zeitparameter der Simulation und in b.) ein umskalierter Zeitparameter verwendet. Die experimentellen Daten sind als Funktion der Bestrahlungszeit aufgetragen.

zeigen damit, wie die verschiedenen Untersuchungsmethoden dieser Arbeit zusammengeführt werden. Für den Vergleich der Topographien und der spektralen Leistungsdichte sei auf die vorangegangenen Kapitel verwiesen. In Abb. 9.1 ist für die experimentellen Daten die tatsächliche Bestrahlungszeit verwendet worden. Für die Simulationsdaten ist in Abb. 9.1.a der Zeitparameter der Simulation verwendet worden, während in Abb. 9.1.b die Zeit so umskaliert worden ist, dass die Rauigkeitsentwicklung von Experiment und Kontinuumsmodell nach gleicher Zeit den Wert minimaler Rauigkeit erreicht. Eine Umskalierung der Zeit ändert die relativen Beiträge der deterministischen Prozesse sowie den Exponenten $\beta$ nicht. Bisher sind die Parameter der Kontinuumsgleichung aus den Ergebnissen der MD-Simulationen für CuTi abgeleitet worden. Eine entsprechende Auswertung der MD-Simulationen für Si liefert für beide Koeffizienten der deterministischen Terme und insbesondere den, der zum Term des Oberflächenfließens gehört, dem Betrage nach deutlich kleinere Werte mit $c_{2}=6.36 \cdot 10^{-3} \mathrm{~nm}^{2} / \mathrm{s}$ und $c_{4}=-5.23 \cdot 10^{-2} \mathrm{~nm}^{4} / \mathrm{s}$. Damit würde der Glättungsprozess entsprechend länger dauern. Die Ergebnisse zeigen somit, dass die Glättung für $\mathrm{CuTi}$ am schnellsten und für Si am langsamsten verläuft und $\mathrm{Zr}_{65} \mathrm{Al}_{7.5} \mathrm{Cu}_{27.5}$ sich bezüglich der Rauigkeitsentwicklung zwischen beiden Systemen befindet. So lässt sich eine Umskalierung wie in Abb. 9.1.b rechtfertigen. Weil für Si das Verhältnis $c_{2} / c_{4}$ dem Betrage nach größer ist als für $\mathrm{CuTi}$, sollte sich in einer Simulation mit den Parametern für Si ein kleinerer Wert für den Exponenten $\zeta$ in den spektralen Leistungsdichten ergeben. Damit ist also auch bezüglich der spektralen Leistungsdichten eine Annäherung an die experimentellen Ergebnisse zu erwarten. Möglich wäre auch, die Koeffizienten durch eine direkte Anpassung an die experimentellen Daten weiter zu verbessern.

In der Modellierung der strahlungsinduzierten Prozesse sind zunächst Terme in li- 
nearer Ordnung berücksichtigt worden. Von Untersuchungen des Wachstums dünner Schichten ist bekannt, dass der Anfangsbereich durch ausschließlich lineare Terme gut beschrieben werden kann. Bei der Untersuchung der strahlungsinduzierten Glättung herrschen allerdings andere Verhältnisse: Es wird eine Oberfläche untersucht, die vor Beginn der Bestrahlung rau und strukturiert und am Ende glatt ist. Insofern sollten nichtlineare Terme zu Beginn nicht zwangsläufig vernachlässigbar sein, weil nichtlineare Terme an Bedeutung gewinnen, wenn die Höhenmodulation groß ist. Andererseits sollten bei Glättungsprozessen, wie im Folgenden erläutert, die nichtlinearen Effekte insgesamt nicht so wichtig sein wie bei Wachstumsprozessen. Beim Wachstum mit dominierenden Aufrauungsprozessen sind die nichtlinearen Terme dafür verantwortlich, dass die Rauigkeit ab einer gewissen Schichtdicke, bei der nichtlineare Effekte an Gewicht gewinnen, nicht mehr so deutlich ansteigt wie im Anfangsbereich und somit nicht ins Unendliche steigt [BS95]. Bei der Glättung durch Bestrahlung nimmt die Rauigkeit ab, so dass im stationären Grenzfall einer glatten Schicht die linearen Terme dominieren. Für den qualitativ zu erwartenden und auch experimentell bestätigten Verlauf sind somit für den stationären Grenzfall keine nichtlinearen Terme in der Beschreibung notwendig.

Die Abgrenzung verschiedener Prozesse und die quantitative Bestimmung ihrer Verhältnisse untereinander durch MD-Simulationen kann für die Interpretation einer Vielzahl von Experimenten, die sich mit strahlungsinduzierter Strukturbildung beschäftigen, herangezogen werden. Von besonderem Interesse sind die Ergebnisse für Computermodelle, in die die Physik der relevanten Prozesse als Annahme eingeht. Als solche sind neben den hier untersuchten Kontinuumsmodellen auch Monte Carlo Simulationen zu nennen, denn dort muss die Art der möglichen atomaren Dynamik vorgegeben werden.

Ein in der Literatur diskutierter Effekt, der in den MD-Simulationen nicht bearbeitet worden ist, ist der der sogenannten Sputter-Redeposition [DZLW00]. Dies ist ein Glättungseffekt, der darauf beruht, dass sich gesputterte Atome auf benachbarten Hügeln niederlassen können. Dieser Effekt lässt sich in MD-Simulationen nur dann bearbeiten, wenn in der Simulation die Oberfläche aus Hügeln besteht. Um bezüglich der Energie und Längenskalen realistisch zu bleiben, sollten die Hügel von ähnlicher Größe wie im Experiment sein, d.h. ungefähr $20 \mathrm{~nm}$ Durchmesser und ungefähr $10 \mathrm{~nm}$ Höhendifferenz zwischen Hügel und Tal. Um einige Hügel auf der Oberfläche anzuordnen, wären Kantenlängen der Zelle im Bereich von $80 \mathrm{~nm}$ sinnvoll. Das entspricht gegenüber den hier verwendeten Zellen einer Vergrößerung um den Faktor 10 in $\mathrm{x}$ - und y-Richtung. Auch wenn die Zelle in z-Richtung nicht so sehr vergrößert wird, steigt die Anzahl der Atome auf mehr als das hundertfache an. Damit ist der Rechenaufwand sehr groß, weil außerdem eine Vielzahl von Einschüssen simuliert werden muss, um statistisch signifikante Ergebnisse zu erzielen. Durch die MD-Simulationen in dieser Arbeit, die an glatten Oberflächen bei verschiedenen Auftreffwinkeln durchgeführt wurden, konnte gezeigt werden, dass die durch Sputtererosion bedingte Aufraung gegenüber dem Prozess des ballistischen Glättens vernachlässigbar ist. Der Effekt der Sputter-Redeposition sollte für eine eher glatte Oberfläche einen kleineren Einfluss als der der Sputtererosion haben, weil aufgrund der geometrischen Verhältnisse bei einer eher glatten Schicht es für ein abgelöstes Atom wahrscheinlicher ist, die Oberfläche ganz zu verlassen, als auf einen benachbarten Hügel zu treffen. Im Rahmen einer Kontinuumsmodellierung ist 
ein Sputter-Redepositionseffekt im Zusammenhang mit einem linearen Dämpfungsterm gemäß Gleichung (4.29) diskutiert worden [FBS ${ }^{+}$04]. In Kap. 8.3 hat sich herausgestellt, dass ein solcher Term keinen wesentlichen Beitrag in den vorgestellten Experimenten haben sollte. Somit scheint insgesamt für die hier betrachteten Strukturen und Bestrahlungsenergien die Sputter-Redeposition kein Effekt von wesentlicher Bedeutung zu sein.

Die Einordnung der Ergebnisse bezüglich der verwendeten Bestrahlungsenergie hat zwei wesentliche Aspekte. Das sind zum einen das Endresultat und zum anderen die beitragenden wesentlichen Mechanismen. Das Endresultat bei der hier verwendeten Energie $E=3 \mathrm{keV}$ ist sehr ähnlich zu dem bei der wesentlich höheren Energie $E=1.8 \mathrm{MeV}$ [MA01]. Ionenbestrahlung kann somit auch im leicht zugänglichen Energiebereich einiger keV zur Glättung von Oberflächen eingesetzt werden. Als beitragende Mechanismen konnten für die kleine Energie aber eindeutig andere als bei der hohen Energie identifiziert werden. Für die Modellierung der Ionenbestrahlung ist die Bestrahlungsenergie somit ein Parameter, der die relative Relevanz der teilnehmenden Prozesse bestimmt. Deshalb ergeben sich je nach Energie verschiedene Modelle zur Beschreibung der Strukturentwicklung. Entscheidend ist dabei der Übergang des Fließprozesses vom Volumenfließen bei hoher Energie zum Oberflächenfließen bei niedriger Energie. Eine krümmungsinduzierte Oberflächendiffusion spielt nach den Ergebnissen aus dieser Arbeit und aus [MA01] weder bei Energien im keV Bereich noch bei Energien im MeV Bereich eine bedeutende Rolle.

Für Abweichungen zwischen den Experimenten und den MD-Simulationen kommen zwei Unterschiede in Frage. Einerseits werden in den MD-Simulationen andere, wenngleich sehr ähnliche, Materialien als im Experiment verwendet und zum anderen finden die MD-Simulationen auf anderen Zeitskalen als die Experimente statt. Die unterschiedliche Zeitskala sollte bei den betrachteten möglichen Prozessen lediglich bei der Diffusion einen Einfluss haben. Die Kombination zwischen experimentellen Daten und MD-Simulationen in Kap. 7.1.3.2 schlägt gerade die Brücke zwischen beiden Zeitskalen und zeigt, dass auch die Diffusion in der MD-Simulation realistisch abgeschätzt werden kann. Somit sind verschiedene Zeitskalen kein Grund für Abweichungen zwischen Experiment und MD-Simulation. Die Verwendung unterschiedlicher Materialsysteme in Experiment und Simulation ist sicherlich ein Grund für Abweichungen. Ebenso sind immer Abweichungen zwischen Experiment und MD-Simulation zu erwarten, weil die in der klassischen MD-Simulation verwendeten empirischen Potentiale nur eine Näherung der realen Verhältnisse darstellen können. Die Verwendung von zwei Extrembeispielen glasbildender Systeme führt zwar einerseits zu Abweichungen zum experimentell verwendeten System, aber andererseits ergeben sich aus übereinstimmenden Ergebnissen der beiden Extrembeispiele Aussagen, die für eine größere Klasse von Gläsern gültig sind.

Dass es leichte quantitative Unterschiede zwischen dem experimentell verwendeten System und den in der Simulation verwendeten gibt, stellt keine größere Schwierigkeit dar, wenn das verfolgte Ziel die Identifikation der beitragenden Prozesse ist. Die MDSimulationen zeigen gerade, dass die Relevanz der beitragenden Prozesse qualitativ nicht von der speziellen Struktur des Materialsystems abhängig ist. 


\subsection{Vergleich mit anderen Materialsystemen}

Da die Untersuchungen zur Relevanz der verschiedenen Prozesse in den MD-Simulationen für zwei sehr unterschiedliche amorphe Materialsysteme durchgeführt worden sind und dabei für beide Materialien sehr ähnliche Ergebnisse gewonnen werden konnten, ist davon auszugehen, dass sich ähnliche Ergebnisse auch für andere amorphe Systeme, die mit ihren Eigenschaften zwischen den hier untersuchten Systemen liegen, ergeben. So gesehen sollten die hier gewonnenen Ergebnisse für viele andere Materialien von Bedeutung sein.

In der Literatur wird neben Glättung durch Ionenbestrahlung unter einer Vielzahl von Bedingungen auch über Aufrauung berichtet. Deshalb wird im Folgenden der Zusammenhang hergestellt, in dem die verschiedenen Experimente und Beobachtungen stehen.

Das quantitative Verhältnis der an der Strukturentwicklung teilnehmenden Prozesse lässt sich durch die Wahl des Materials, der Ionensorte, der Bestrahlungsenergie, der Umgebungstemperatur und der Bestrahlungsgeometrie beeinflussen.

Vielfach untersucht sind kristalline Halbleiteroberflächen. Bei nicht zu hohen Temperaturen wird durch die Bestrahlung der Oberflächenbereich amorphisiert $\left[\mathrm{FDK}^{+} 99\right.$; ZFHR05], so dass die Ergebnisse dieser Experimente mit den in dieser Arbeit durchgeführten Untersuchungen verglichen werden können. Für strukturierte Ge Schichten konnte für Bestrahlung mit Xe ${ }^{+}$Ionen der Energie $1 \mathrm{keV}$ unter schrägem Einfall ebenfalls gezeigt werden, dass die Ausgangsstrukturen geglättet werden [CMK ${ }^{+}$94]. Bei höheren Temperaturen wie $300{ }^{\circ} \mathrm{C}$ wurde dagegen eine Aufrauung beobachtet. Der Einfluss der Temperatur liegt dabei darin, dass bei der niedrigeren Temperatur die Oberfläche durch die Bestrahlung amorphisiert, während sie bei der höheren Temperatur kristallin ist. Für verschiedene Halbleitersysteme ist bekannt, dass durch das Zusammenspiel von glättenden und aufrauenden Mechanismen Strukturbildung stattfindet, die bei schrägem Beschuss zur Bildung wellenartiger Korrugationen (Ripple) [NSC62] und bei senkrechtem Beschuss zur Bildung von runden Hügelstrukturen führt [FDK ${ }^{+}$99; FSB00]. Auch das hier in der MD-Simulation untersuchte Silizium ist experimentell hinsichtlich des Einflusses von Ionenbestrahlung im niedrigen $\mathrm{keV}$ Bereich für verschiedene Ionensorten untersucht worden [ZFHR05]. Dabei konnte für Bestrahlungswinkel im Bereich um $45^{\circ}$ relativ zur Substratnormalen Dominanz der Glättung gefunden werden [ $\mathrm{FFF}^{+} 04$; ZFHR05]. Wenn der Bestrahlungswinkel nahezu $0^{\circ}$ relativ zur Substratnormalen beträgt, wird dagegen Aufrauung beobachtet. Diese Aufrauung findet aber erst bei sehr hohen Fluenzen der Größenordnung $10^{18}$ Ionen $/ \mathrm{cm}^{2}$ statt. Dementsprechend wurde von Petersen [Pet07] im Winkelbereich zwischen $20^{\circ}$ und $50^{\circ}$ bei der Bestrahlung von Si mit $\mathrm{Kr}^{+}$-Ionen der Energie $5 \mathrm{keV}$ bis zu einer Fluenz von $10^{18}$ Ionen $/ \mathrm{cm}^{2}$ stets glatte Oberflächen beobachtet. Bis zu den in dieser Arbeit untersuchten Fluenzen ist somit auch beim senkrechten Beschuss von Silizium noch keine starke Aufrauung ursprünglich glatter Schichten zu erwarten. In dieser Arbeit konnte ein Glättungseffekt bei kleineren Fluenzen gefunden werden, der ähnlich zu dem von Ge Schichten beobachteten ist [CMK ${ }^{+}$94].

Vom kristallinen metallischen Systemen einer Ni Schicht wird berichtet, dass Bestrahlung im niedrigen keV Bereich auf kleinen Skalen zu einer Aufrauung und auf großen lateralen Skalen zu einer Glättung führt $\left[\mathrm{CFM}^{+} 96\right]$. Auch in diesem System gibt es demnach 
ein Wechselspiel zwischen aufrauenden und glättenden Mechanismen, und die relative Relevanz der Effekte ist von der lateralen Strukturgröße abhängig. Dass das Glätten im kristallinen metallischen System nicht so eindeutig ist, könnte daran liegen, dass Fließprozesse im kristallinen System einen kleineren Beitrag als im Amorphen zeigen. In MD-Simulationen zur Glättung von Nanopartikeln auf Substraten amorpher bzw. kristalliner Metallsysteme konnte gezeigt werden [ZAAA03], dass der Glättungsmechanismus für amorphe Systeme effizienter als für kristalline ist. Kristalline Substrate tendieren außerdem mehr zu verstärkten Kavitationseffekten, die mit sogenannten Mikroexplosionen verbunden sind. Deshalb ist der Aufrauungseffekt bei kristallinen Substraten tendenziell größer als bei amorphen.

Insgesamt lassen sich somit die hier gewonnenen Ergebnisse hinsichtlich Übereinstimmungen und Differenzen zu in der Vergangenheit untersuchten Systemen einordnen. 


\section{Zusammenfassung und Ausblick}

Insgesamt konnten in dieser Arbeit grundlegende Effekte und Mechanismen, die bei der Bestrahlung amorpher Oberflächen mit Ionen im Energiebereich weniger keV von Bedeutung sind, untersucht bzw. identifiziert werden. Es hat sich erwiesen, dass eine strukturierte Oberfläche durch Ionenbestrahlung im Energiebereich weniger keV geglättet werden kann. Des Weiteren konnten die wesentlichen Prozesse, die die Entwicklung der Oberflächenstruktur bestimmen, weitgehend identifiziert werden. Dadurch ist eine Modellierung der niederenergetischen Bestrahlung strukturierter amorpher Oberflächen durch stochastische Ratengleichungen möglich und hier auch durchgeführt worden. Die Resultate tragen somit zum tieferen Verständnis der Strukturentwicklung auf mesoskopischer Skala durch auf atomistischer Skala stattfindende Effekte bei. Insbesondere bleibt festzuhalten, dass die Glättung durch einen $\nabla^{4} h$-Term im Kontinuumsmodell auf einen Fließprozess und nicht auf einen diffusiven Prozess zurückzuführen ist. Dies gilt, wie die MD-Simulationen zeigen, sowohl für das metallische Glas $\mathrm{CuTi}$, in dem die Einschüsse „thermal spikes“ hervorrufen, als auch für amorphes Si, in dem es keine „thermal spikes“ ähnlicher Größe gibt. Außerdem konnte hier erstmals der ballistische Glättungsprozess in Verbindung mit weiteren Glättungsprozessen in die Untersuchungen einbezogen werden.

Die Tatsache, dass eine Glättung beobachtet wird, ist ähnlich zu den Ergebnissen, die für die Bestrahlung strukturierter Oberflächen im MeV Energiebereich bekannt sind. Die Untersuchungen der vorliegenden Arbeit zeigen, dass sich allerdings die Mechanismen, die zur Glättung beitragen, zwischen den Energiebereichen keV und $\mathrm{MeV}$ unterscheiden. Für die Anwendung interessant ist vor allem der insgesamt resultierende Effekt, dass auch im experimentell leicht zugänglichen Energiebereich weniger keV eine Glättung strukturierter Oberflächen möglich ist.

Weiterhin sind die in dieser Arbeit gewonnenen Erkenntisse von Bedeutung für die Modellierung von Strukturbildungsprozessen, die durch Ionenbestrahlung getrieben sind. Da Strukturbildung immer das Resultat aus einem Zusammenspiel von glättenden und aufrauenden Mechanismen ist, können die hier erhaltenen Ergebnisse zur relativen Relevanz der glättenden Prozesse untereinander verwendet werden.

In Abgrenzung zu den durch Ionenbestrahlung getriebenen Prozessen haben MDSimulationen Aufschluss über die Art der atomaren Dynamik auf Oberflächen und im Probeninneren bei thermischer Anregung liefern können. Hier hat sich für das metallische Glas an den Oberflächen eine Tendenz zu Einzelsprüngen der kleineren Atomsorte gezeigt. Durch Variation der Zusammensetzung konnten außerdem frühere experimentelle Arbeiten weitergehend interpretiert werden.

In sämtlichen MD-Simulationen sind die grundlegenden Prozesse für typische Vertreter metallischer und kovalent gebundener Halbleiter-Gläser untersucht worden. Das bezüglich der Bestrahlung experimentell untersuchte System steht in seinen Eigenschaf- 
ten zwischen den in den MD-Simulationen untersuchten Systemen, so dass Gemeinsamkeiten der Materialien aus der MD-Simulation auch für das experimentelle System gültig sind. Diese gemeinsamen, grundlegenden Eigenschaften konnten hier identifiziert werden.

Für zukünftige Untersuchungen interessant wäre die Untersuchung weiterer Bestrahlungsenergien im Bereich zwischen der hier untersuchten Energie und dem MeV Energiebereich. Dadurch wäre die genauere Charakterisierung des Übergangs vom Oberflächenfließen im keV Energiebereich zum Volumenfließen im MeV Energiebereich als Funktion der Bestrahlungsenergie möglich.

Nachdem hier metallische und kovalente Systeme untersucht worden sind und sich qualitativ ähnliche Resultate ergeben haben, wäre eine Ausdehnung der Untersuchungen auf Systeme mit ionischen Bindungsanteilen wie z.B. Oxide wünschenswert. Damit könnte das Verständnis des Einflusses der Bindungsart auf die Oberflächenprozesse vertieft werden. Als weitere Materialklasse für die Untersuchung von Glättungsmechanismen bieten sich Polymere an, deren Bausteine um ein Vielfaches größer als die der bisher erwähnten Systeme sind. Für Polymere wären allerdings Anregungsmethoden nötig, die die Ketten nicht zerstören. 


\section{Anhang}

\subsection{Oberflächenfließen im Ortsraum}

In [SL88] wird die Stokes-Gleichung für den Fall gelöst, dass die flüssige Schicht nur entlang einer Richtung auf der Oberfläche gekrümmt ist. Im dreidimensionalen Fall gilt allgemein [LL66]:

$$
\eta \Delta \vec{v}=\nabla p
$$

mit der Geschwindigkeit $\vec{v}$, dem Druck $p$ und der Viskosität $\eta$. Analog zu [SL88] wird für dünne Flüssigkeitsfilme angenommen, dass die Bedingungen der „lubrication approximation" erfüllt sind, d.h. insbesondere gilt:

$$
\begin{aligned}
\frac{\partial p}{\partial z} & =0 \\
\left|\frac{\partial^{2} v_{i}}{\partial x^{2}}\right| & \ll\left|\frac{\partial^{2} v_{i}}{\partial z^{2}}\right| \quad \text { mit } i=x, y \\
\left|\frac{\partial^{2} v_{i}}{\partial y^{2}}\right| & \ll\left|\frac{\partial^{2} v_{i}}{\partial z^{2}}\right| \quad \operatorname{mit} i=x, y .
\end{aligned}
$$

Damit ergeben sich analog zu [SL88] für 2-dimensionale Substrate die Gleichungen

$$
\begin{aligned}
\eta \frac{\partial^{2} v_{x}}{\partial z^{2}} & =\frac{\partial p}{\partial x} \\
\eta \frac{\partial^{2} v_{y}}{\partial z^{2}} & =\frac{\partial p}{\partial y} .
\end{aligned}
$$

In der Näherung kleiner Steigungen $(|\nabla h| \ll 1)$ erstreckt sich der flüssige Bereich von $z=$ $-a_{z}$ bis $z=0$. Die Gleichungen lassen sich integrieren und liefern wegen Gleichung (11.2) und den Randbedingungen, dass an der Grenzfläche fest - flüssig die Geschwindigkeiten null sind $\left(v_{i}\left(z=-a_{z}\right)=0, i=x, y\right)$ und an der Grenzfläche flüssig - Vakuum keine Scherkräfte wirken (entsprechend $\frac{\partial v_{i}}{\partial z}(z=0)=0$ für $i=x, y$ wegen der Annahmen der „lubrication approximation" [SL88]):

$$
\begin{aligned}
& v_{x}=\frac{1}{\eta} \frac{\partial p}{\partial x}\left(\frac{1}{2} z^{2}-\frac{1}{2} a_{z}^{2}\right) \\
& v_{y}=\frac{1}{\eta} \frac{\partial p}{\partial y}\left(\frac{1}{2} z^{2}-\frac{1}{2} a_{z}^{2}\right) .
\end{aligned}
$$


Die Höhenänderungsrate ergibt sich nun aus der Kontinuitätsgleichung mit $\nabla=\left(\frac{\partial}{\partial x}, \frac{\partial}{\partial y}\right)$ :

$$
\begin{aligned}
\frac{\partial h}{\partial t} & =-\nabla \int_{-a_{z}}^{0}\left(v_{x}, v_{y}\right) d z \\
& =-\nabla\left[\nabla p \frac{1}{\eta} \int_{-a_{z}}^{0}\left(\frac{1}{2} z^{2}-\frac{1}{2} a_{z}^{2}\right) d z\right] \\
& =\frac{a_{z}^{3}}{3 \eta} \nabla(\nabla p) \\
& =-\frac{\gamma a_{z}^{3}}{3 \eta} \nabla^{4} h .
\end{aligned}
$$

Im letzten Schritt ist ausgenutzt worden, dass in Abwesenheit von Scherspannungen in der hier verwendeten Näherung $p=-\gamma \nabla^{2} h$ gilt [LL66]. 


\subsection{Einfluss der Glättungsprozesse auf die Entwicklung der Rauigkeit}

Der Schritt von Gleichung (4.35) zu Gleichung (4.36) ergibt sich für die verschiedenen Ordnungen $j \geq 1$ wie folgt.

$$
j=1 \text { : }
$$

$$
\begin{aligned}
\sigma^{2} & =2 \pi C \int_{0}^{\infty} q e^{-2 b_{1} q t} d q \\
& =\frac{2 \pi C}{\left(2 b_{1} t\right)^{2}} \text { nach [BSMM97] }
\end{aligned}
$$

$j=2$ :

$$
\begin{aligned}
\sigma^{2} & =2 \pi C \int_{0}^{\infty} q e^{-2 b_{2} q^{2} t} d q \\
& =2 \pi C \frac{1}{2} \int_{0}^{\infty} e^{-2 b_{2} u t} d u \text { mit } u=q^{2} \\
& =\left[-\frac{\pi C}{2 b_{2} t} e^{-2 b_{2} u t}\right]_{0}^{\infty} \\
& =\frac{\pi C}{2 b_{2} t}
\end{aligned}
$$

$j=3:$

$$
\begin{aligned}
\sigma^{2} & =2 \pi C \int_{0}^{\infty} q e^{-2 b_{3} q^{3} t} d q \\
& =2 \pi C \frac{2}{3} \int_{0}^{\infty} u^{-1 / 3} e^{-2 b_{3} u t} d u \text { mit } u=q^{3} \\
& =\frac{2}{3} \pi C \frac{\Gamma(2 / 3)}{\left(2 b_{3} t\right)^{2 / 3}}
\end{aligned}
$$

mit der Gamma-Funktion $\Gamma$ nach [BSMM97]

$j=4:$

$$
\begin{aligned}
\sigma^{2} & =2 \pi C \int_{0}^{\infty} q e^{-2 b_{4} q^{4} t} d q \\
& =2 \pi C \frac{1}{2} \int_{0}^{\infty} e^{-2 b_{4} u^{2} t} d u \text { mit } u=q^{2} \\
& =\pi C \frac{\sqrt{\pi}}{2 \sqrt{2 b_{4} t}} \text { nach [BSMM97] }
\end{aligned}
$$


Für einen Prozess der Ordnung $j=0$ ergibt sich Gleichung (4.37) aus Gleichung (4.35) wegen

$$
\begin{aligned}
\sigma^{2} & =2 \pi C \int_{0}^{\infty} q e^{-2 b_{0} t} d q \\
& =2 \pi C e^{-2 b_{0} t} \int_{0}^{\infty} q d q .
\end{aligned}
$$

Mit den verwendeten Integrationsgrenzen divergiert das Integral in der letzten Zeile von Gleichung (11.17). Ersetzt man die obere Grenze durch einen endlichen, beliebig großen Wert - das entspricht wegen begrenzter Aufösung auch den Bedingungen im Experiment und der Computersimulation -, so ergibt sich ein exponentieller Zerfall der Rauigkeit. 


\subsection{Beitrag des Rauschens zur Rauigkeit}

Im Folgenden wird gezeigt, dass der Beitrag des Rauschens zur Rauigkeit von der Struktur abhängig ist. Als Modell wird eine in einer Dimension periodische Höhenfunktion betrachtet:

$$
h(x)=B \sin (q x) .
$$

Für die Rauigkeit dieser Struktur $\left(\sigma_{i}\right)$ ergibt sich:

$$
\begin{aligned}
\sigma_{i} & =\sqrt{\left\langle h^{2}\right\rangle-\langle h\rangle^{2}} \\
& =\sqrt{B^{2}\left\langle\sin ^{2}(q x)\right\rangle-0} \\
& =B / \sqrt{2} .
\end{aligned}
$$

Wird zu einer solchen periodischen Struktur, die sich in kleine räumliche Intervalle aufteilen lässt, in jedem Intervall mit gleicher Wahrscheinlichkeit entweder die Höhe $d$ addiert oder subtrahiert, so entspricht das einem Rauschen und für die Rauigkeit $\left(\sigma_{f}\right)$ ergibt sich:

$$
\begin{aligned}
\sigma_{f} & =\sqrt{\frac{1}{2}\left\langle B^{2} \sin ^{2}(q x)+d^{2}+2 d B \sin (q x)\right\rangle+\frac{1}{2}\left\langle B^{2} \sin ^{2}(q x)+d^{2}-2 d B \sin (q x)\right\rangle} \\
& =\sqrt{\frac{B^{2}}{2}+d^{2}} .
\end{aligned}
$$

Für den durch das Rauschen bedingten Rauigkeitszuwachs ergibt sich:

$$
\sigma_{f}-\sigma_{i}=\sqrt{\frac{B^{2}}{2}+d^{2}}-\frac{B}{\sqrt{2}}
$$

und damit im Grenzfall sehr rauer Schichten $(B \gg d)$

$$
\sigma_{f}-\sigma_{i}=0
$$

und im Grenzfall sehr glatter Schichten $(B \ll d)$

$$
\sigma_{f}-\sigma_{i}=d
$$

Der durch das Rauschen bedingte Rauigkeitszuwachs ist somit umso größer, je glatter die gesamte Struktur ist. 


\subsection{Volumenfließen im Ortsraum}

Um zu zeigen, dass der in Gl. (8.8) untersuchte Term die mittlere Höhe konstant hält und damit Teilchenzahl-erhaltend ist, genügt es, zu zeigen, dass $\int_{\text {Oberfläche }} \frac{\partial h}{\partial t} d x d y=0$ gilt. Die Gültigkeit dieser Beziehung wird im Folgenden gezeigt:

$$
\begin{aligned}
\int_{\mathrm{OF}} \frac{\partial h}{\partial t} d x d y= & \tilde{c}_{1} \int_{\mathrm{OF}}\left(h-\langle h\rangle_{A}\right)|\nabla h| d x d y \\
= & \tilde{c}_{1} \int\left[|\nabla h| \cdot \int\left(h-\langle h\rangle_{A}\right) d x-\int\left(\int\left(h-\langle h\rangle_{A}\right) d x\right) \frac{\partial}{\partial x}|\nabla h| d x\right] d y \\
= & \tilde{c}_{1}\left[\int\left(h-\langle h\rangle_{A}\right) d x d y \cdot \int|\nabla h| d y\right] \\
& -\tilde{c}_{1}\left[\int\left(h-\langle h\rangle_{A}\right) d x d y \cdot \frac{\partial}{\partial x}|\nabla h|-\int\left(\int\left(h-\langle h\rangle_{A}\right) d x d y\right) \cdot \frac{\partial}{\partial x \partial y}|\nabla h| d y\right] \\
= & 0 .
\end{aligned}
$$

Die Integration erfolgt jeweils über die Oberfläche der Substratkoordinaten. Der Übersichtlichkeit wegen ist der Index am Integral ab der zweiten Zeile nicht mehr aufgeschrieben. Der letzte Schritt gilt wegen

$$
\int\left(\left(h-\langle h\rangle_{A}\right) d x d y=0 .\right.
$$


11 Anhang

180 


\section{Literaturverzeichnis}

[AB81] Andersen, H. H. ; BAY, H. L.: Sputtering Yield Measurements. In: Sputtering by Particle Bombardment I. Springer Verlag, Berlin Heidelberg, 1981

[AR98] Averback, R. S. ; Rubia, T. Diaz De L.: Displacement Damage in Irradiated Metals and Semiconductors. In: Solid State Physics 51, 281 (1998)

[ASG+97] Arndt, M. ; Stannarius, R. ; Groothues, H. ; Hempel, E. ; Kremer, F.: Length Scale of Cooperativity in the Dynamic Glass Transition. In: Phys. Rev. Lett. 79, 2077 (1997)

[AT87] Allen, M. P. ; Tildesley, D. J.: Computer Simulation of Liquids. Oxford, UK : Clarendon Press, 1987

[BBJ98] Busch, R. ; BAKke, E. ; Johnson, W. L.: Viscosity of the supercooled liquid and relaxation at the glass transition of the $\mathrm{Zr}_{46.75} \mathrm{Ti}_{8.25} \mathrm{Cu}_{7.5} \mathrm{Ni}_{10} \mathrm{Be}_{27.5}$ bulk metallic glass forming alloy. In: Acta Mater. 46, 4725 (1998)

[Böd99] BöDDEKER, B.: Oberflächennahe Dynamik amorpher, freistehender, metallener $N i_{0,5} Z r_{0,5}$-Filme in der Molekulardynamik-Simulation, Universität Göttingen, Dissertation, 1999

[BEH $\left.{ }^{+} 01\right]$ Busse, C. ; Engin, C. ; Hansen, H. ; Linke, U. ; Michely, T. ; UrbasSEK, H. M.: Adatom formation and atomic layer growth on $\mathrm{Al}(111)$ by ion bombardment: experiments and molecular dynamics simulations. In: Surf. Sci. 488, 346 (2001)

[BH54] Buckel, W. ; Hilsch, R.: Einfluß der Kondensation bei tiefen Temperaturen auf den elektrischen Widerstand und die Supraleitung für verschiedene Metalle. In: Z. Physik 138, 109 (1954)

[BH88] Bradley, R. M. ; Harper, J. M. E.: Theory of ripple topography induced by ion bombardment. In: J. Vac. Sci. Technol. A 6, 2390 (1988)

[Bon93] Bonnell, D.A.: Scanning Tunneling Microscopy and Spectroscopy: Theory, Techniques and Applications. New York: VCH, 1993

[BPG $\left.{ }^{+84}\right]$ Berendsen, H. J. C. ; Postma, J. P. M. ; van Gunsteren, W. F. ; DiNola, A. ; HaAk, J. R.: Molecular dynamics with coupling to an external bath. In: J. Chem. Phys. 81, 3684 (1984) 
[Bre96] Brenig, W.: Statistische Theorie der Wärme. Berin, Heidelberg : Springer Verlag, 1996

[BRGW82] Binnig, G. ; Rohrer, H. ; Gerber, Ch. ; Weibel, E.: Surface studies by scanning tunneling microscopy. In: Phys. Rev. Lett. 49, 5 (1982)

[BS95] Barabasi, A.-L. ; Stanley, H. E.: Fractal Concepts in Surface Growth. Cambridge, UK : Cambridge University Press, 1995

[BSMm97] Bronstein, I. N. ; Semendjajew, K. A. ; Musiol, G. ; Mühlig, H.: Taschenbuch der Mathematik. Frankfurt am Main : Verlag Harri Deutsch, 1997

[BSS96] Bhuiyan, G. M. ; Silbert, M. ; Stott, M. J.: Structure and thermodynamic properties of liquid transition metals: An embedded-atom-method approach. In: Phys. Rev. B 53, 636 (1996)

[BT99] BöDdeker, B. ; Teichler, H.: Dynamics near free surfaces of molecular dynamics simulated $\mathrm{Ni}_{0.5} \mathrm{Zr}_{0.5}$ metallic glass films. In: Phys. Rev. E 59, 1948 (1999)

[Car98] Carter, G.: Sample rotation and reduced surface roughening in oblique incidence ion sputtering erosion. In: Vacuum 49, 285 (1998)

[CC93] Cook, S. J. ; Clancy, P.: Comparison of semi-empirical potential functions for silicon and germanium. In: Phys. Rev. B 47, 7686 (1993)

[CFM+96] Csahok, Z. ; Farkas, Z. ; Menyhard, M. ; Gergely, G. ; Daroczi, Cs. S.: Surface morphology development during ion sputtering: roughening or smoothing? In: Surf. Sci. Lett. 364, L600 (1996)

[CMK $\left.{ }^{+} 94\right]$ Chason, E. ; Mayer, T. M. ; Kellerman, B. K. ; McIlroy, D. T. ; HowArD, A. J.: Roughening Instability and Evolution of the Ge(001) Surface during Ion Sputtering. In: Phys. Rev. Lett. 72, 3040 (1994)

[Com04] Computer Graphic Service. RBS Analysis and Simulation Package [v. 4.00(beta)] Revision Level: Version 0.950. Nov 2004

[Cra75] CRAnk, J.: The Mathematics of Diffusion. Oxford, UK : Oxford University Press, 1975

[Cul78] Cullity, B. D.: Elements of x-ray diffraction, 2nd edition. Reading, UK : Addison-Wesley, 1978

[CV96] Carter, G. ; Vishnyakov, V.: Roughening and ripple instabilities on ion-bombarded Si. In: Phys. Rev. B 54, 17647 (1996) 
[DB83] Daw, M. S. ; BAskes, M. I.: Semiempirical, quantum mechanical calculation of hydrogen embrittlement in metals. In: Phys. Rev. Lett. 50, 1285 (1983)

[DB84] DAw, M. S. ; BAskes, M. I.: Embedded-atom method: Derivation and application to impurities, surfaces, and other defects in metals. In: Phys. Rev. B 29, 6443 (1984)

[Doo86] Doolittle, L. R.: A Semiautomatic Algorithm for Rutherford Backscattering Analysis. In: Nuc. Instr. and Meth. B 15, 227 (1986)

[DZLW00] Drotar, J. T. ; Zhao, Y.-P. ; Lu, T.-M. ; Wang, G.-C.: Mechanisms for plasma and reactive ion etch-front roughening. In: Phys. Rev. B 61, 3012 (2000)

[EAC $\left.{ }^{+} 99\right]$ Erlebacher, J. ; Aziz, M. J. ; Chason, E. ; Sinclair, M. B. ; Floro, J. A.: Spontaneous Pattern Formation on Ion Bombarded Si(001). In: Phys. Rev. Lett. 82, 2330 (1999)

[Ed106] EDLER, T.: Mechanische Spannungen beim Beschuss von Germanium mit Edelgasionen: Simulationen und Experimente, Universität Göttingen, Diplomarbeit, 2006

[El190] Elliott, S. R.: Physics of Amorphous Materials. UK : Longmann Scientific \& Technical, 1990

[EM07] EdLeR, T. ; MAYR, S. G.: Mechanisms of stress generation during bombardment of Ge with keV ions: Experiments and molecular dynamics simulation. In: New J. Phys., submitted (2007)

[EWF $\left.{ }^{+} 93\right]$ Eaglesham, D. J. ; White, A. E. ; Feldman, L. C. ; Moriya, N. ; Jacobson, D. C.: Equilibrium Shape of Si. In: Phys. Rev. Lett. 70, 1643 (1993)

[FBD86] Folles, S. M. ; Baskes, M. I. ; DAw, M. S.: Embedded-atom-method functions for the fcc metals $\mathrm{Cu}, \mathrm{Ag}, \mathrm{Au}, \mathrm{Ni}, \mathrm{Pd}, \mathrm{Pt}$, and their alloys. In: Phys. Rev. B 33, 7983 (1986)

[FBS $\left.{ }^{+} 04\right]$ Facsko, S. ; Bobek, T. ; Stahl, A. ; Kurz, H. ; Dekorsky, T.: Dissipative continuum model for self-organized pattern formation during ion-beam erosion. In: Phys. Rev. B 69, 153412 (2004)

[FDK $\left.{ }^{+} 99\right]$ Facsko, S. ; Dekorsky, T. ; Koerdt, C. ; Trappe, C. ; Kurz, H. ; Vogt, A. ; Hartnagel, H. L.: Formation of Ordered Nanoscale Semiconductor Dots by Ion Sputtering. In: Science 285, 1551 (1999) 
[FFF ${ }^{+}$04] Frost, F. ; Fechner, R. ; Flamm, D. ; Ziberi, B. ; Frank, W. ; SchindLER, A.: Ion beam assisted smoothing of optical surfaces. In: Appl. Phys. A 78, 651 (2004)

[FFM ${ }^{+}$03] Faupel, F. ; Frank, W. ; Macht, M.-P. ; Mehrer, H. ; Naundorf, V. ; RÄtzke, K. ; Schober, H. R. ; Sharma, S. K. ; Teichler, H.: Diffusion in metallic glasses and supercooled melts. In: Rev. Mod. Phys. 75, 237 (2003)

[FGS ${ }^{+}$03] Foffi, G. ; Götze, W. ; Sciortino, F. ; Tartaglia, P. ; Voigtmann, Th.: Mixing Effects for the Structural Relaxation in Binary Hard-Sphere Liquids. In: Phys. Rev. Lett. 91, 085701 (2003)

[FL89] Fortner, J. ; LAnNin, J. S.: Radial distribution functions of amorphous silicon. In: Phys. Rev. B 39, 5527 (1989)

[Foi85] FoIles, S. M.: Application of the embedded-atom method to liquid transition metals. In: Phys. Rev. B 32, 3409 (1985)

[FSB00] Frost, F. ; Schindler, A. ; Bigl, F.: Roughness Evolution of Ion Sputtered InP Surfaces: Pattern Formation and Scaling Laws. In: Phys. Rev. Lett. 85, 4116 (2000)

[FV85] Family, F. ; VicseK, T.: Scaling of the active zone in the Eden process on percolation networks and the ballistic deposition model. In: J. Phys. A 18, L75 (1985)

[GHK98] GeYeR, U. ; VON HÜlSEN, U. ; Kopf, H.: Internal interfaces and intrinsic stress in thin amorphous $\mathrm{Cu}-\mathrm{Ti}$ and $\mathrm{Co}-\mathrm{Tb}$ films. In: J. Appl. Phys. 83, 3065 (1998)

[GKS99] Gaukel, G. ; Kluge, M. ; Schober, H. R.: Diffusion and relaxations in liquid and amorphous metals. In: Phil. Mag. B 79, 1907 (1999)

[GT01] Guerdane, M. ; Teichler, H.: Structure of the amorphous, massivemetallic-glass forming $\mathrm{Ni}_{25} \mathrm{Zr}_{60} \mathrm{Al}_{15}$ alloy from molecular dynamics simulations. In: Phys. Rev. B 65, 014203 (2001)

[Haa94] HaAsen, P.: Physikalische Metallkunde. Berlin, Heidelberg : Springer Verlag, 1994

[Her50] Herring, C.: Effect of Change of Scale on Sintering Phenomena. In: $J$. Appl. Phys. 21, 301 (1950)

[HH91] Hamann, C. ; Hietschold, M.: Raster-Tunnel-Mikroskopie. Berlin : Akademie Verlag, 1991 
[HIKS05] Hara, S. ; Izumi, S. ; Kumagai, T. ; Sakai, S.: Surface energy, stress and structure of well-relaxed amorphous silicon: A combination approach of ab initio and classical molecular dynamics. In: Surf. Sci. 585, 17 (2005)

[HK94] Hafner, J. ; KraJCI, M.: Propagating and localized vibrational modes in Ni-Zr glasses. In: J. Phys.: Condens. Matter 6, 4631 (1994)

[HKW04] Hedler, A. ; Klaumünzer, S. L. ; Wesch, W.: Amorphous silicon exhibits a glass transition. In: Nature Mat. 3, 804 (2004)

[Hü198] VON HÜLSEn, U.: Schichtwachstum, Relaxation und Kristallisation kokondensierter amorpher CuTi-Schichten, Universität Göttingen, Dissertation, 1998

[HS04] Hu, Y. ; SinnotT, S. B.: Constant temperature molecular dynamics simulations of energetic particle-solid collisions: comparison of temperature control methods. In: J. Comp. Phys. 200, 251 (2004)

[HT87] Hansma, P. K. ; Tersoff, J.: Scanning tunneling microscopy. In: J. Appl. Phys. 61, R1 (1987)

[Hud98] Hudson, J. B.: Surface Science, An Introduction. New York : Wiley Interscience, 1998

[IKT ${ }^{+}$94] Inoue, A. ; Kawase, D. ; Tsai, A. P. ; Zhang, T. ; Matsumoto, T.: Stability and transformation to crystalline phases of amorphous $\mathrm{Zr}-\mathrm{Al}-\mathrm{Cu}$ alloys with significant supercooled liquid region. In: Mater. Sci. Eng. A 178, 255 (1994)

[IMm97] Ishimaru, M. ; Munetoh, S. ; Motooka, T.: Generation of amorphous silicon structures by rapid quenching: A molecular-dynamics study. In: Phys. Rev. B 56, 15133 (1997)

[IZM92] Inoue, A. ; Zhang, T. ; Matsumoto, T.: The structural relaxation and glass transition of La-Al-Ni and Zr-Al-Cu amorphous alloys with a significant supercooled liquid region. In: J. Non-Cryst. Solids 150, 396 (1992)

[KDD $\left.{ }^{+} 93\right]$ Kneller, E. ; Du, C. ; Dümpelmann, D. ; Fröchte, B. ; Khan, Y. ; Sostarich, M.: Glass Temperature and Solid - Liquid Transition of Metallic Glasses. In: Z. Metallkd. 84, 574 (1993)

[KHS86] Klaumünzer, S. ; Hou, M.-D. ; Schumacher, G.: Coulomb Explosions in a Metallic Glass Due to the Passage of Fast heavy Ions? In: Phys. Rev. Lett. 57, 850 (1986)

[Kie31] KiEssig, H.: Interferenz von Röntgenstrahlen an dünnen Schichten. In: Annalen der Physik 5/10 (7), 769 (1931) 
[Kit93] KitTeL, C.: Einführung in die Festkörperphysik. München : R. Oldenbourg Verlag, 1993

[KMAB93] Köster, U. ; Meinhardt, J. ; Aronin, A. ; Birol, Y.: Crystallization of $\mathrm{Cu}_{50} \mathrm{Ti}_{50}$ Glasses and Undercooled Melts. In: Z. Metallkd. 84, 574 (1993)

[KMP ${ }^{+}$89] Kugler, S. ; Molnar, G. ; Petö, G. ; Zsoldos, E. ; Rosta, L. ; MeNelle, A. ; Bellissent, R.: Neutron-diffraction study of evaporated pure amorphous silicon. In: Phys. Rev. B 40, 8030 (1989)

[KPS91] Kloeden, P.E. ; Platen, E. ; Schurz, H.: Numerical Solution of Stochastic Differential Equations through Computer Experiments. Berlin, Heidelberg : Springer Verlag, 1991

[KS04] Kluge, M. ; Schober, H. R.: Diffusion and jump length distribution in liquid and amorphous $\mathrm{Cu}_{33} \mathrm{Zr}_{67}$. In: Phys. Rev. B 70, 224209 (2004)

[KSM+84] Kamitakahara, W. A. ; Shanks, H. R. ; McClelland, J. F. ; Gompf, F. ; Pintschovius, L.: Measurement of Phonon Densities of States for Pure and Hydrogenated Amorphous Silicon. In: Phys. Rev. Lett. 52, 644 (1984)

[KTH91] Kubo, R. ; Toda, M. ; Hashitsume, N.: Statistical Physics II, Nonequilibrium Statistical Physics. Berlin, Heidelberg : Springer Verlag, 1991

[LL66] Landau, L. D. ; Lifschitz, E. M.: Lehrbuch der theoretischen Physik, Band VI, Hydrodynamik. Berlin : Akademie-Verlag, 1966

[LL88] Luedtke, W. D. ; Landman, U.: Preparation and melting of amorphous silicon by molecular-dynamics simulations. In: Phys. Rev. B 37, 4656 (1988)

[LWRW84] Lindgren, S. A. ; Wallden, L. ; Rundgren, J. ; Westrin, P.: Lowenergy electron diffraction from $\mathrm{Cu}(111)$ : Subthreshold effect and energydependent inner potential; surface relaxation and metric distances between spectra. In: Phys. Rev. B 29, 576 (1984)

[MA01] Mayr, S. G. ; Averback, R. S.: Surface Smoothing of Rough Amorphous Films by Irradiation-Induced Viscous Flow. In: Phys. Rev. Lett. 87, 196106 (2001)

[MAaA03] Mayr, S. G. ; Ashrenazy, Y. ; Albe, K. ; Averback, R. S.: Mechanisms of Radiation-Induced Viscous Flow: Role of Point Defects. In: Phys. Rev. Lett. 90, 055505 (2003)

[May97] MayR, S. G.: Oberflächenrauhigkeit von amorphen ZrAlCu Schichten, Universität Augsburg, Diplomarbeit, 1997 
[May00] MaYR, S. G.: Wachstum amorpher Schichten: Vergleich von Experiment und Simulation im Bereich Oberflächenrauhigkeit und mechanische Spannungen, Universität Göttingen, Dissertation, 2000

[May03] MaYR, S. G. private Mitteilung. 2003

[May05a] MAYR, S. G.: Impact of ion irradiation on the thermal, structural, and mechanical properties of metallic glasses. In: Phys. Rev. B 71, 144109 (2005)

[May05b] MaYR, S. G. private Mitteilung. 2005

[MCB02] Makeev, M. A. ; Cuerno, R. ; Barabasi, A.-L.: Morphology of ionsputtered surfaces. In: Nucl. Instrum. Methods Phys. Res. B 197, 185 (2002)

[MCH94] Mayer, T. M. ; Chason, E. ; Howard, A. J.: Roughening instability and ion-induced viscous relaxation of $\mathrm{SiO}_{2}$ surfaces. In: J. Appl. Phys. 76, 1633 (1994)

[MGC $\left.{ }^{+} 05\right]$ Moseler, M. ; Gumbsch, P. ; Casiraghi, C. ; Ferrari, A. C. ; RoBeRTson, J.: The Ultrasmoothness of Diamond-like Carbon Surfaces. In: Science 309, 1545 (2005)

[MKWl91] Mo, Y. W. ; Kleiner, J. ; WebB, M. B. ; Lagally, M. G.: Activation Energy of Surface Diffusion of Si on $\mathrm{Si}(001)$ : A Scanning-TunnelingMicroscopy Study. In: Phys. Rev. Lett. 66, 1998 (1991)

[MMS99] Mayr, S. G. ; Moske, M. ; SAmwer, K.: Identification of key parameters by comparing experimental and simulated growth of vapor-deposited amorphous $\mathrm{Zr}_{65} \mathrm{Al}_{7.5} \mathrm{Cu}_{27.5}$ films. In: Phys. Rev. B 60, 16950 (1999)

[Mor96] Mortimer, C. E.: Chemie - Das Basiswissen der Chemie. Stuttgart : Georg Thieme Verlag, 1996

[MT94] Michely, T. ; Teichert, C.: Adatom yields, sputtering yields and damage patterns of single-ion impacts on $\operatorname{Pt}(111)$. In: Phys. Rev. B 50, 11156 (1994)

[Mul57] Mullins, W. W.: Theory of Thermal Grooving. In: J. Appl. Phys. 28, $333(1957)$

[Mul59] Mullins, W. W.: Flattening of a Nearly Plane Solid Surface due to Capillarity. In: J. Appl. Phys. 30, 77 (1959)

[NA97] Nordlund, K. ; Averback, R. S.: Point defect movement and annealing in collision cascades. In: Phys. Rev. B 56, 2421 (1997) 
[NGA+98] Nordlund, K. ; Ghaly, M. ; Averback, R. S. ; Caturla, M. ; de la Rubia, T. D. ; TARus, J.: Defect production and collision cascades in elemental semiconductors and fcc metals. In: Phys. Rev. B 57, 7556 (1998)

[Nor95] NordLund, K.: Molecular dynamics simulation of ion ranges in the 1-100 keV energy range. In: Comp. Mat. Sci. 3, 448 (1995)

[NSC62] Navez, M. ; Sella, C. ; Chaperot, D.: Étude de l'attaque du verre par bombardement ionique. In: Compt. Rend. Acad. Sci. 254, 240 (1962)

[Oec73] Oechsner, H.: Untersuchungen zur Festkörperzerstäubung bei schiefwinkligem Ionenbeschuß polykristalliner Metalloberflächen im Energiebereich um 1 keV. In: Z. Physik 261, 37 (1973)

[Orc62] Orchard, S. E.: On Surface Levelling in Viscous Liquids and Gels. In: Appl. Sci. Res. A 11, 451 (1962)

[OS99] Oligschleger, C. ; Schober, H. R.: Collective jumps in a soft-sphere glass. In: Phys. Rev. B 59, 811 (1999)

[Pai56] PAI, S.-I.: Viscous Flow Theory I - Laminar Flow. Princeton, New Jersey : D. van Nostrand Company, Inc., 1956

[Par54] Parratt, L. G.: Surface studies of solids by total reflection of x-rays. In: Phys. Rev. 95, 359 (1954)

[Pet07] Petersen, J. private Mitteilung. 2007

[PNM81] Puska, M. J. ; Nieminen, R. M. ; Manninen, M.: Atoms embedded in electron gas: Immersion energies. In: Phys. Rev. B 24, 3037 (1981)

[PT96] Press, W. ; Tolan, M.: Spekuläre und nicht-spekuläre Reflexion von Röntgen- und Neutronenstrahlen. In: 27. IFF-Ferienkurs, Streumethoden zur Untersuchung kondensierter Materie. Forschungszentrum Jülich GmbH, 1996

[PTVF94] Press, W.H. ; Teukolsky, S.A. ; Vetterling, W.T. ; Flannery, B.P.: Numerical Recipes in C: The Art of Scientific Computing. Cambridge, UK : Cambridge University Press, 1994

[Rai00] RAIBLE, M.: Stochastische Feldgleichungen für amorphes Schichtwachstum, Universität Augsburg, Dissertation, 2000

[Rap95] Rapaport, D. C.: The Art of Molecular Dynamics Simulation. Cambridge, UK : Cambridge University Press, 1995

[Rei97] ReINkeR, B. M.: STM-Untersuchungen an amorphen ZrCo- und ZrAlCuAufdampfschichten, Universität Augsburg, Dissertation, 1997 
[RG95] DE la Rubia, T. D. ; Gilmer, G. H.: Structural Transformations and Defect Production in Ion Implanted Silicon: A Molecular Dynamics Simulation Study. In: Phys. Rev. Lett. 74, 2507 (1995)

$\left[\mathrm{RML}^{+} 00\right]$ Raible, M. ; Mayr, S. G. ; Linz, S. J. ; Moske, M. ; Hänggi, P. ; SAMWER, K.: Amorphous thin film growth: Theory compared with experiment. In: Europhys. Lett. 50, 61 (2000)

[RMS97] Reinker, B. ; Moske, M. ; SAmwer, K.: Kinetic roughening of amorphous $\mathrm{Zr}_{65} \mathrm{Al}_{7.5} \mathrm{Cu}_{27.5}$ films investigated in situ with scanning tunneling microscopy. In: Phys. Rev. B 56, 9887 (1997)

[RVR $\left.{ }^{+} 82\right]$ Rasigni, G. ; Varnier, F. ; Rasigni, M. ; Palmari, J. P. ; Llebaria, A.: Autocovariance functions, root-mean-square-roughness height, and autocovariance length for rough deposits of copper, silver and gold. In: Phys. Rev. B 25, 2315 (1982)

[Sch06] Schroeder, K.: Ab initio Molecular Dynamics. In: 37th IFF Spring School, Computational Condensed Matter Physics. Forschungszentrum Jülich GmbH, 2006

[SCP91] Stich, I. ; CAR, R. ; PARrinello, M.: Amorphous silicon studied by ab initio molecular dynamics: Preparation, structure and properties. In: Phys. Rev. B 44, 11092 (1991)

[Seg73] SegmülleR, A.: Observation of x-ray interferences on thin films of amorphous silicon. In: Thin Solid Films 18, 287 (1973)

[Sev73] Sevchik, N. J.: Growth instabilities in the deposition of amorphous films. In: J. Non. Cryst. Solids 12, 141 (1973)

[SGB80] Suck, J.-B. ; Güntherodt, H.-J. ; BeCK, H.: Short-wavelength transverse collective modes in a metallic glass. In: J. Phys. C: Sol. St. Phys. 13, L1045 (1980)

[Sig69] Sigmund, P.: Theory of Sputtering. I. Sputtering Yield of Amorphous and Polycrystalline Targets. In: Phys. Rev. 184, 383 (1969)

[SK56] Seitz, F. ; Koehler, J. S.: Displacement of Atoms during Irradiation. In: Solid State Physics 2, 305 (1956)

[SL88] Stillwagon, L. E. ; Larson, R. G.: Fundamentals of topographic substrate leveling. In: J. Appl. Phys. 63, 5251 (1988)

[SL91] SABochick, M. J. ; LAM, N. Q.: Radiation induced amorphization of ordered intermetallic compounds $\mathrm{CuTi}, \mathrm{CuTi}_{2}$ and $\mathrm{Cu}_{4} \mathrm{Ti}_{3}$ : A moleculardynamics study. In: Phys. Rev. B 43, 5243 (1991) 
[SR83] Suck, J.-B. ; Rudin, H.: Vibrational Dynamics of Metallic Glasses Studied by Neutron Inelastic Scattering. In: Glassy Metals II. Springer Verlag, Berlin Heidelberg, 1983

[SSM02] Streng, C. ; SAmwer, K. ; Mayr, S. G.: Breakdown of the approximations of small perturbations in continuum modeling of amorphous thin film growth. In: Appl. Phys. Lett. 81, 5135 (2002)

[SSM06] Streng, C. ; SAmwer, K. ; Mayr, S. G.: Surface smoothing and pattern formation in the transient region of compositionally modulated amorphous metallic alloy films: Experiment versus theory. In: Phys. Rev. B 73, 104107 (2006)

[Str04] Streng, C.: Wachstumsanalyse amorpher dicker Schichten und Schichtsysteme, Universität Göttingen, Dissertation, 2004

[SW85] Stillinger, F. H. ; Weber, T. A.: Computer simulation of local order in condensed phases of silicon. In: Phys. Rev. B 31, 5262 (1985)

[SW92] Schatz, G. ; Weidinger, A.: Nukleare Festkörperphysik. Stuttgart : B. G. Teubner, 1992

[Tei92] Teichler, H.: Configuration Dynamics in a Simulated Finite-Temperature Transition Metal Glass. In: Phys. Stat. Sol. (b) 172, 325 (1992)

[Tei01] Teichler, H.: Structural dynamics on the $\mu$ s scale in molecular-dynamics simulated, deeply undercooled, glass-forming $\mathrm{Ni}_{0.5} \mathrm{Zr}_{0.5}$. In: J. Non-Cryst. Solids 293-295, 339 (2001)

[Tei05] Teichler, H.: Heterogeneous dynamics on the microsecond scale in simulated $\mathrm{Ni}_{0.5} \mathrm{Zr}_{0.5}$ metallic melts far below the glass temperature. In: Phys. Rev. E 71, 031505 (2005)

[Ter86] Tersoff, J.: New Empirical Model for the Structural Properties of Silicon. In: Phys. Rev. Lett. 56, 632 (1986)

[Ter88a] Tersoff, J.: Empirical interatomic potential for silicon with improved elastic properties. In: Phys. Rev. B 38, 9902 (1988)

[Ter88b] Tersoff, J.: New empirical approach for the structure and energy of covalent systems. In: Phys. Rev. B 37, 6991 (1988)

[Teu62] Teufer, G.: The crystal structure of tetragonal $\mathrm{ZrO}_{2}$. In: Acta Cryst. 15, $1187(1962)$

[TH85] Tersoff, J. ; Hamann, D. R.: Theory of the scanning tunneling microscope. In: Phys. Rev. B 31, 805 (1985) 
[TM77] Tyson, W. R. ; Miller, W. A.: Surface Free Energies Of Solid Metals: Estimation From Liquid Surface Tension Measurements. In: Surf. Sci. 62, 267 (1977)

[TW94] Tong, W. M. ; Williams, R. S.: Kinetics of Surface Growth: Phenomenology, Scaling, and Mechanisms of Smoothening and Roughening. In: Annu. Rev. Phys. Chem. 45, 401 (1994)

[UHC01] Umbach, C. C. ; Headrick, R. L. ; Chang, K.-C.: Spontaneous Nanoscale Corrugation of Ion-Eroded $\mathrm{SiO}_{2}$ : The Role of Ion-Irradiation-Enhanced Viscous Flow. In: Phys. Rev. Lett. 87, 246104 (2001)

[UPB $\left.{ }^{+} 85\right]$ Uhrmacher, M. ; Pampus, K. ; Bergmeister, F. J. ; Purschke, D. ; LIEB, K. P.: Energy calibration of the $500 \mathrm{kV}$ heavy ion implanter IONAS. In: Nuc. Instr. and Meth. B 9, 234 (1985)

[Vau02] VAuth, S.: Experimente und Simulationen zum Wachstum amorpher Aufdampfschichten bei schräger Teilchendeposition, Universität Göttingen, Diplomarbeit, 2002

[Vic92] VicseK, T.: Fractal Growth Phenomena. Singapore : World Scientific, 1992

[Vil91] Villain, J.: Continuum models of crystal growth from atomic beams with and without desorption. In: J. Phys. I 1, 19 (1991)

[VL05] Vogel, S. ; Linz, S. J.: Continuum modeling of sputter erosion under normal incidence: Interplay between nonlocality and nonlinearity. In: Phys. Rev. B 72, 035416 (2005)

[VM05] Vauth, S. ; Mayr, S. G.: Atomic dynamics in molecular dynamics simulations of glassy CuTi thin films. In: Appl. Phys. Lett. 86, 061913 (2005)

[VM07] VAuth, S. ; MAYR, S. G.: Relevance of surface viscous flow, surface diffusion, and ballistic effects in $\mathrm{keV}$ ion smoothing of amorphous surfaces. In: Phys. Rev. B 75, 224107 (2007)

[Vol91] Volkert, C. A.: Stress and plastic flow in silicon during amorphization by ion bombardment. In: J. Appl. Phys. 70, 3521 (1991)

[VSmS03] Vauth, S. ; Streng, C. ; Mayr, S. G. ; Samwer, K.: Growth of vapordeposited amorphous $\mathrm{Zr}_{65} \mathrm{Al}_{7.5} \mathrm{Cu}_{27.5}$ films under oblique particle incidence investigated by experiment and simulation. In: Phys. Rev. B 68, 205425 (2003)

[VVarV06] Valladares, A. ; Valladares, R. M. ; Alvarez-Ramírez, F. ; ValLADARES, A. A.: Studies of phonon densitiy of states in ab initio generated amorphous structures of pure silicon. In: J. Non-Cryst. Solids 352, 1032 (2006) 
[Wah91] WahnströM, G.: Molecular-dynamics study of a supercooled twocomponent Lennard-Jones system. In: Phys. Rev. A 44, 3752 (1991)

[Was80] WasedA, Y.: The Structure of Non-Crystalline Materials. New York : McGraw-Hill, 1980

[Wei98] WeIss, M.: Mechanische Eigenschaften und thermische Stabilität tief unterkühlter Metallschmelzen im Bereich der Glastemperatur, Universität Augsburg, Dissertation, 1998

[Win06] Winkler, R. G.: Molecular Dynamics Simulations. In: 37th IFF Spring School, Computational Condensed Matter Physics. Forschungszentrum Jülich GmbH, 2006

[YZWL96] Yang, H.-N. ; ZhaO, Y.-P. ; Wang, G.-C. ; Lu, T.-M.: Noise-Induced Roughening Evolution of Amorphous Si films Grown by Thermal Evaporation. In: Phys. Rev. Lett. 76, 3774 (1996)

[ZAAA03] Zhong, Y. ; Ashrenazy, Y. ; Albe, K. ; Averback, R. S.: Ion beam smoothening of metal surfaces. In: J. Appl. Phys. 94, 4432 (2003)

[Zar91] Zarzycki, J.: Glasses and the Vitreous State. Cambridge, UK : Cambridge University Press, 1991

[ZB03] Ziegler, J. F. ; Biersack, J. P. SRIM computer code, Version 2003.20. 2003

[ZBL85] Ziegler, J. F. ; Biersack, J. P. ; Littmark, U.: The Stopping and Range of Ions in Matter. New York : Pergamon Press, 1985

[ZFHR05] Ziberi, B. ; Frost, F. ; Höche, Th. ; Rauschenbach, B.: Ripple pattern formation on silicon surfaces by low-energy ion-beam erosion: Experiment and theory. In: Phys. Rev. B 72, 235310 (2005) 


\section{Publikationen}

2003: S. Vauth, C. Streng, S. G. Mayr, and K. Samwer:

Growth of vapor-deposited amorphous $\mathrm{Zr}_{65} \mathrm{Al}_{7.5} \mathrm{Cu}_{27.5}$ films under oblique particle incidence investigated by experiment and simulation,

Phys. Rev. B 68, 205425 (2003)

2004: J. Hachenberg, C. Streng, E. Süske, S. Vauth, S. G. Mayr, H.-U. Krebs, and K. Samwer:

Kinetic Roughening of Laser Deposited Polymer Films:

Crossover from Single Particle to Continuous Growth,

Phys. Rev. Lett. 92, 246102 (2004)

2005: S. Vauth and S. G. Mayr:

Atomic dynamics in molecular dynamics simulations of glassy CuTi thin films, Appl. Phys. Lett. 86, 061913 (2005)

2007: S. Vauth and S. G. Mayr:

Relevance of surface viscous flow, surface diffusion, and ballistic effects in keV ion smoothing of amorphous surfaces,

Phys. Rev. B 75, 224107 (2007)

S. Vauth and S. G. Mayr:

Smoothing of vapor-deposited amorphous $\mathrm{Zr}_{65} \mathrm{Al}_{7.5} \mathrm{Cu}_{27.5}$ films

by $\mathrm{keV}$ ion irradiation,

in preparation for Phys. Rev. B 


\section{Danksagung}

Mein besonderer Dank gilt Herrn Prof. Dr. Stefan G. Mayr für die Ermöglichung, dieses sehr interessante Thema im Rahmen der Dissertation zu bearbeiten und für die Betreuung dieser Arbeit. Sein Interesse am Fortschritt der Arbeit war stets sehr groß und hat sich in zahlreichen Diskussionen und Anregungen gezeigt. Weiterhin war auch seine Unterstützung bezüglich technischer Schwierigkeiten stets hilfreich.

Herrn Prof. Dr. Hans-Ulrich Krebs danke ich für seinen Einsatz als Korreferent und sein Interesse an der Arbeit.

Auch Herrn Prof. Dr. Konrad Samwer möchte ich für das Interesse an dieser Arbeit danken, das sich in verschiedenen Seminaren gezeigt hat.

Bei Hayo Zutz und Herrn Prof. Dr. H. Hofsäss bedanke ich mich für die Durchführung der RBS-Messungen.

Dem SFB 602 vertreten durch Gerda Brocks und Regina Hühne gilt mein Dank für die finanzielle Unterstützung.

Bei technischen Schwierigkeiten sowie Computerproblemen konnte ich mich stets auf Carsten Mahn verlassen, wofür ich mich bedanken möchte. Auch der Feinmechanischen Werkstatt gilt mein Dank für ihre Unterstützung.

Für das Korrekturlesen der Arbeit bedanke ich mich bei meinen Kollegen Christian Vree, Dennis Bedorf, Tobias Edler und Dr. Jörg Hachenberg.

Für eine stets gute Atmosphäre im Büro gilt mein Dank Christian Vree, Dr. Jörg Hachenberg und Melanie Schneider, sowie für die ersten Monate meiner Zeit als Doktorand im schönen Altbau Kai Gehrke und Dr. Henning Harms. Auch allen übrigen Mitgliedern des Instituts, die ich während meiner Zeit kennen gelernt habe, danke ich dafür, dass sie stets zum Spaß bei und/oder neben der Arbeit beigetragen haben.

Meinen Freunden außerhalb des Instituts gilt mein Dank für die manchmal nötige Ablenkung von der Physik.

Meinen Eltern danke ich für ihre Unterstützung, auf die ich mich stets verlassen kann. Weiterhin danke ich auch meinem Bruder für das aufmerksame Korrekturlesen der Arbeit aus der Sicht eines nicht zu sehr physikalisch Vorbelasteten. 


\section{Lebenslauf}

\section{Angaben zur Person:}

Name:

Geburtstag:

Geburtsort:

Familienstand:

Staatsangehörigkeit:

\section{Schulbildung:}

1982-1986:

1986-1995:

1995:

\section{Zivildienst:}

Juni 1995 - Juni 1996:

\section{Studium:}

WS 1996/1997 - WS 2002/2003:

November 1998:

WS 1999/2000:

November 2002:

seit SS 2003:

seit Januar 2003:

\author{
Sebastian Vauth \\ 15.04.1976 \\ Rahden \\ ledig \\ Deutsch
}

Freiherr-vom-Stein-Grundschule, Lübbecke

Wittekind-Gymnasium, Lübbecke

Abitur (Note: 1,3)

Lebenshilfe Lübbecke e.V

Diplomstudiengang Physik an der

Georg-August-Universität Göttingen

Vordiplom (Note: 1,15)

Auslandssemester an der Oxford University

Diplom (Note: 1,1)

Diplomarbeit bei Prof. Dr. K. Samwer, Thema:

„Experimente und Simulationen zum Wachstum amorpher Aufdampfschichten bei

schräger Teilchendeposition“

Promotionsstudium im Fach Physik an der Georg-August-Universität Göttingen

Wissenschaftlicher Mitarbeiter im SFB 602 und Anfertigung der vorliegenden Dissertation im I. Physikalischen Institut

Betreuung von Übungsgruppen zur Anfängervorlesung Physik im Haupt- und Nebenfach und zur Festkörperphysik, sowie Betreuung im Fortgeschrittenen-Praktikum

Göttingen, 13.09.2007 